\title{
How to mitigate coal mine bumps through understanding the violent failure of coal specimens
}

\author{
Gamal Rashed
}

Follow this and additional works at: https://researchrepository.wvu.edu/etd

\section{Recommended Citation}

Rashed, Gamal, "How to mitigate coal mine bumps through understanding the violent failure of coal specimens" (2015). Graduate Theses, Dissertations, and Problem Reports. 6479.

https://researchrepository.wvu.edu/etd/6479

This Dissertation is protected by copyright and/or related rights. It has been brought to you by the The Research Repository @ WVU with permission from the rights-holder(s). You are free to use this Dissertation in any way that is permitted by the copyright and related rights legislation that applies to your use. For other uses you must obtain permission from the rights-holder(s) directly, unless additional rights are indicated by a Creative Commons license in the record and/ or on the work itself. This Dissertation has been accepted for inclusion in WVU Graduate Theses, Dissertations, and Problem Reports collection by an authorized administrator of The Research Repository @ WVU.

For more information, please contact researchrepository@mail.wvu.edu. 


\title{
HOW TO MITIGATE COAL MINE BUMPS THROUGH UNDERSTANDING THE VIOLENT FAILURE OF COAL SPECIMENS
}

\author{
Gamal Rashed \\ Dissertation Submitted to the \\ Benjamin M. Statler College of Engineering and Mineral Resources \\ In Partial Fulfilment of the Requirements \\ For the Degree of
}

Doctor of Philosophy

In

Engineering
Syd S. Peng, Ph.D., Committee Chair
Keith A. Heasley, Ph.D.
Gerald L. Finfinger, Ph.D.
Yi Luo, Ph.D.
Brijes Mishra, Ph.D.
Bruce Kang, Ph.D.

\section{Department of Mining Engineering Morgantown, West Virginia 2015}

Keywords: Coal specimen, coal mine bumps, stress-strain curve, violent failure, interface friction, UCS, simulated rock, width-to-height ratio.

Copyright 2015, Gamal Rashed 


\title{
ABSTRACT \\ HOW TO MITIGATE COAL MINE BUMPS THROUGH UNDERSTANDING THE VIOLENT FAILURE OF COAL SPECIMENS
}

\author{
Gamal Rashed
}

Coal mine bumps have presented serious mining problems for many years. These sudden violent failures around underground mine openings have compromised safety, ventilation and access to mine workings. There is general agreement among researchers that both geologic and poor/inappropriate mining conditions that create high stresses in the pillar are the main causative factors for coal bumps.

The main objective of this research is to understand bump mechanisms in laboratory settings through examining the violent failure of coal specimens and to test three hypotheses to mitigate that violent failure. Also, man-made concrete specimens were prepared and tested to simulate failures in underground mines of several geological conditions. To understand and mitigate coal mine bumps, the role of five factors on the potential for violent failure was examined: First, the role of the mechanical properties of coal was investigated. The main reason behind studying the role of the coal itself is that, a debate was found in the literature about that role. From the literature, some researchers think that strong and stiff coal is necessary for coal bumps. However, others think that bumps would happen irrespective of coal strength or stiffness. Hence, the role of the coal was investigated to help put an end to that debate. Second, two of the most commonly proposed bump mechanisms which are sudden loss of constraint and sudden impact load were examined with the aid of Finite Element Method. Many researchers believe that coal bumps occur because of a sudden loss of constraint between pillar/roof \& pillar/floor or the hammering effect due to breaking of thick, massive strata above the coal seam, which creates a sudden impact load on the pillars and causes them to bump, little work has been done to explore and understand the consequences of these proposed mechanisms for coal bumps.

Third, the interface friction and the width-to-height $(\mathrm{W} / \mathrm{H})$ ratio of coal specimens were determined where bump would occur. The $\mathrm{W} / \mathrm{H}$ ratio ranges from 1 to 10 while the interface friction is either 0.1 or 0.25 . It is believed that understanding how the mode of failure of the coal specimen changes with changing interface friction and width-to-height $(\mathrm{W} / \mathrm{H})$ ratio is the corner stone to mitigating coal mine bumps. In the author's point of view, the $\mathrm{W} / \mathrm{H}$ ratio of a pillar is the most convenient way to control coal mine's bump hazard. Fourth, three hypotheses were examined to mitigate the violent failure of coal specimens. These hypotheses depend on softening coal specimens either partially or 
completely. It is believed that softening coal specimens would minimize the elastic and kinetic energies released at the failure.

Fifth, man-made concrete specimens were assembled in a three layered approach to investigate the mode of failure for eight different geological conditions. The main reason for using man-made concrete specimens to simulate different field conditions is that the ability to examine and determine how a specific geologic condition contributes to bumps using actual coal and rock specimens is very difficult, because there is uncertainty about their strength. For man-made concrete specimens the strength is well known before running the tests. The three layers simulate roof, pillar and floor. The proposed strength for the assembled concrete specimens is 2000 \& 4000 psi for weak and strong coal respectively, while the strength is 4000 and 9000 psi for medium and strong-rock respectively.

According to the laboratory and numerical modeling results, the coal itself plays an important role in bumps. However, that role "alone" is not sufficient for the occurrence of bumps. However, the interface friction and the width-to-height ratio generally control the mode of failure for coal specimens. Every interface friction is associated with a specific threshold $\mathrm{W} / \mathrm{H}$ ratio above which the mode of failure is non-violent, and below which the failure would be either sudden \& violent or sudden \& non-violent. The core zone for the failed coal specimens is neither elastic nor intact; however it is damaged to some extent. Softening the rib zone is not effective in mitigating the violent failure of coal specimens. However, softening the core zone would help mitigate that violent failure. This result gives an indication that the energy stored in the core zone of a coal specimen is the main causative factor for the violent failure. Sudden impact load and sudden loss of constraint are associated with instantaneous changes in the vertical stress, and sudden release of elastic and kinetic energy, both the elastic and the kinetic energies are needed for the occurrence of bumps. 


\title{
DEDICATION
}

\author{
To \\ My father, R. Rashed \\ My mother, L. Rashed \\ With their prayer, patience and support the completion of my dissertation would have \\ been possible
}

To

My wife, M. Asar

You have been very patient and helpful all the time,

I cherish everything you have done for me.

To

My advisor, Dr. Syd Peng, I learned uncountable things from you 


\section{ACKNOWLEDGEMENT}

I am very grateful to Dr. Syd S. Peng, my advisor and the committee chair, for his support, contribution, and encouragement. Dr. Peng, was particularly very helpful in guiding me in this entire process of my research. He was very distinguished by asking me challenging questions which require deep thinking. I did my best to answer some of these questions in my dissertation. Besides my advisor, I wish to thank the rest of my dissertation committee members, Dr. Keith Heasley, Dr. Yi Luo, Dr. Brijes Mishra, Dr. Gerald Finfinger, and Dr. Bruce Kang for their advices and corrections towards my thesis and also for their insightful comments and questions.

A special thanks to Kris Lilly, the Assistant of Superintendent of Red Bone Mining, for his wonderful help in providing me the coal samples used in this research. Great thanks are also due to Dave Gearhart and Timothy Batchler from NIOSH, Pitssbugrh, PA for testing some coal specimens for me using the MRS machine. Without their contributions, this research would not have been done. Also, I am thankful to William J. Comstock, the technician for MRB, for his kindness, willingness, and effort in helping me with preparing tools for specimen preparation.

Last but not least, I wish to express my sincere thanks to the department of mining engineering and in particular for providing me with all the necessary facilities for the research. 


\section{TABLE OF CONTENTS}

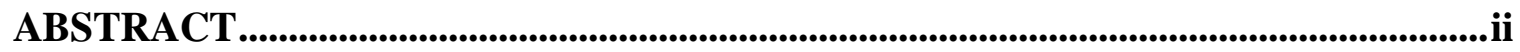

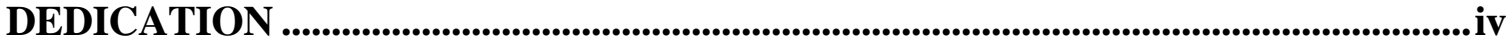

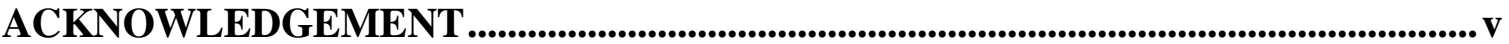

TABLE OF CONTENTS ................................................................................................................. vi

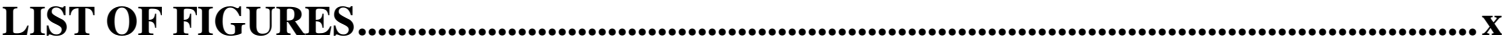

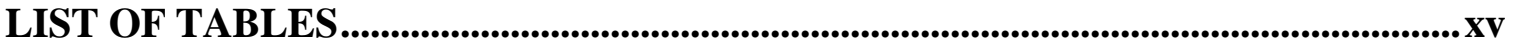

CHAPTER 1： INTRODUCTION

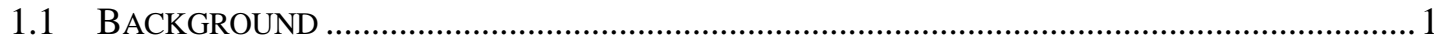

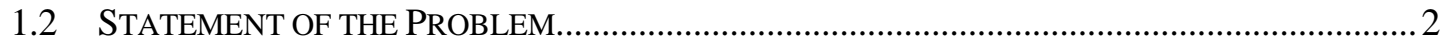

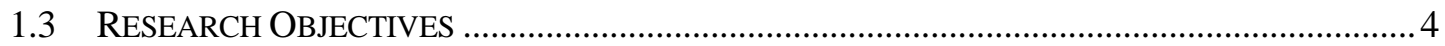

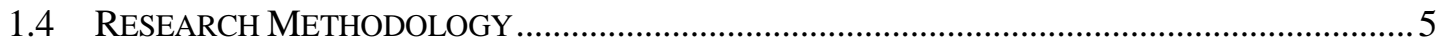

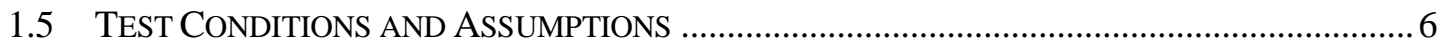

1.6 EXTENDING THE LABORATORY WORK ON COAL SPECIMENS TO COAL PILLARS ............... 7

CHAPTER 2: ITERATURE REVIEW ...........................................................................99

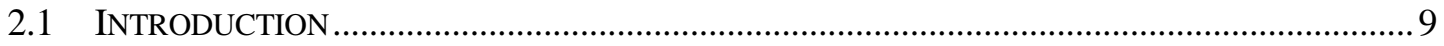

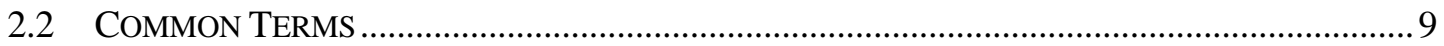

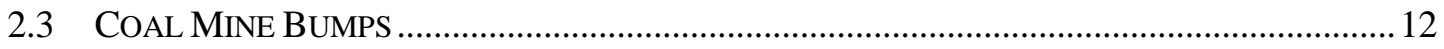

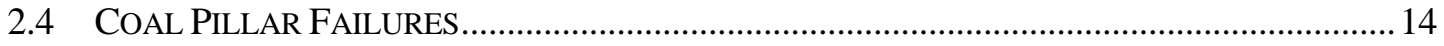

2.5 Geological Conditions AND COAL Mine BuMPS ….................................................. 15

2.6 AN INTERFACE FRICTION AND COAL MINE BUMPS...................................................... 18

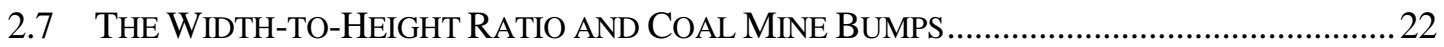




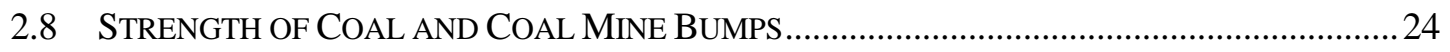

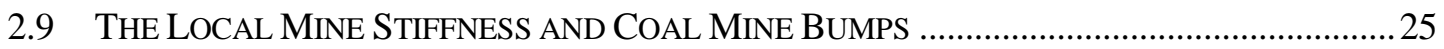

\section{CHAPTER 3: The role of the MECHANICAL PROPERTIES OF COAL IN}

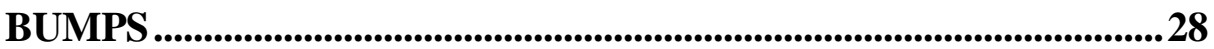

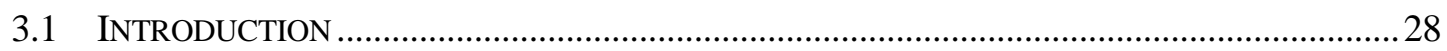

3.2 THE EFFect of UCS AND Young’s MOdulus on the Potential For Violent

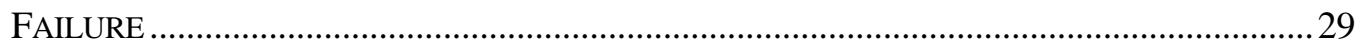

3.3 COMPUTER MODELING FOR THE EFFECT OF UCS ON THE POTENTIAL FOR

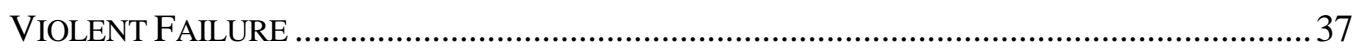

3.4 UCS FOR CYLINDRICAL COAL SPECIMENS FOR THE SUNNYSIDE SEAM, UTAH .................40

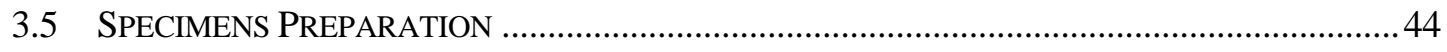

3.6 COMPaRison OF THE Vertical STRENGTH FOR THE SUNNYSIDE SEAM, UtaH AND SEWICKLEY SEAM, WV ………………………………………………………...... 46

3.7 YOUNG'S MODULI FOR THE SUNNYSIDE SEAM, UTAH AND THE SEWICKLEY SEAM, WV

3.8 SHEAR STRENGTH FOR THE SUNNYSIDE SEAM, UtAH COAL AND THE SEWICKLEY

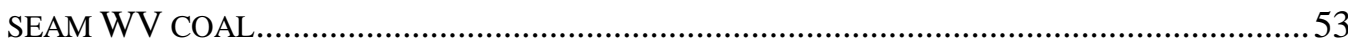

3.9 THE BURST INDEX FOR THE SUNNYSIDE SEAM, UtAH COAL AND THE SEWICKLEY SEAM WV COAL …………………………………………………………......

3.10 SUMMARY FOR CHAPTER THREE ............................................................................... 60

CHAPTER 4: UNDERSTANDING COAL BUMP MECHANISMS...........................62

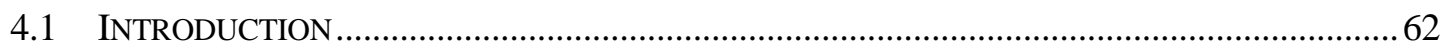

4.2 EXAMINING Why SUdDEN LosS OF Friction Would CAUSE BumPS .............................63

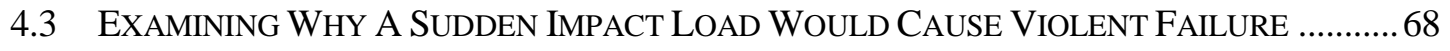




\section{CHAPTER 5: THE ROLE OF INTERFACE FRICTION AND WIDTH-} TO-HEIGHT (W/H) RATIO ON THE FAILURE MODE OF COAL SPECIMENS

5.1 INTRODUCTION. .74

5.2 DETERMINATION OF AN INTERFACE FRICTION 75

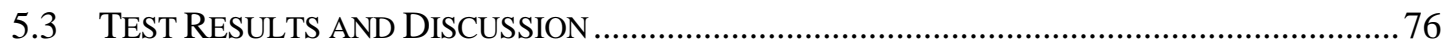

5.4 DETAILED ANALYSIS OF THE RESULTS FOR EACH GROUP............................................... 81

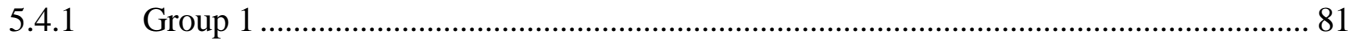

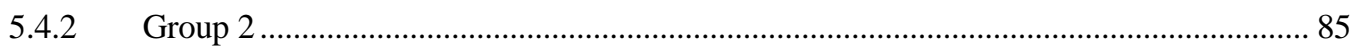

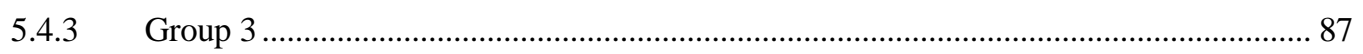

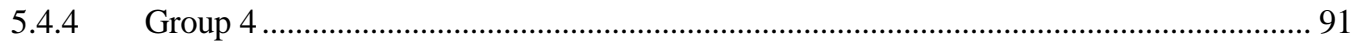

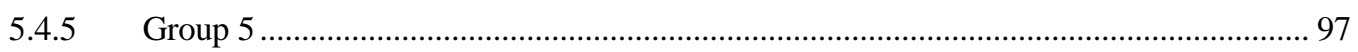

5.5 INVESTIGATING THE FAILED CORE ZONE OF COAL SPECIMEN .......................................... 101

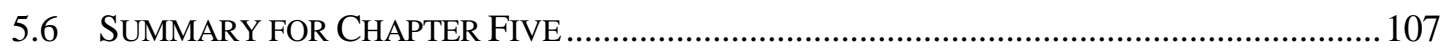

\section{CHAPTER 6: MITIGATIng THE VIOLENT FAILURE OF COAL}

SPECIMENS ...........................................................................................109

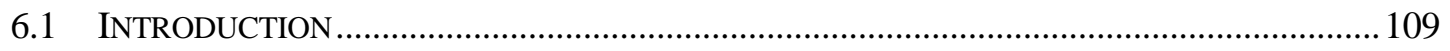

6.2 TeSting the First Hypothesis to Mitigate the Violent Failure ......................... 110

6.3 Testing the Second Hypothesis to Mitigate the Violent Failure...................... 119

6.4 Testing the Third Hypothesis to Mitigate the Violent FaILURE........................ 128

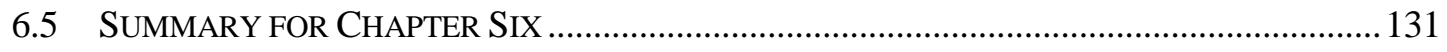

\section{CHAPTER 7: FAILURE OF A SIMULATED LAYERED ROCK}




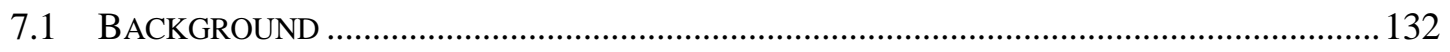

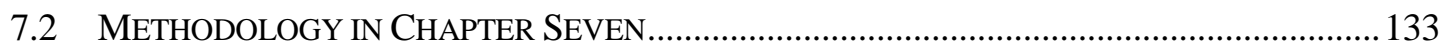

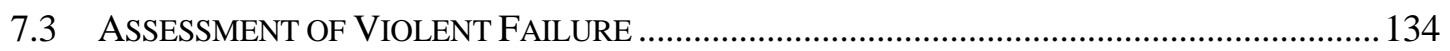

7.4 PhaSE ONE “DEFINITION OF COAL AND Rock STRENGTH” .......................................... 134

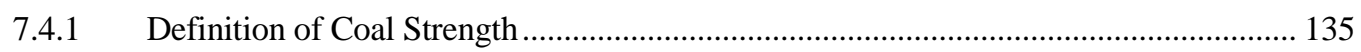

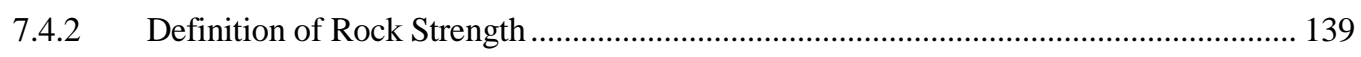

7.5 Phase One “DEVElopment OF MAN-MAdE ConCRETE SPECIMENS” ........................... 141

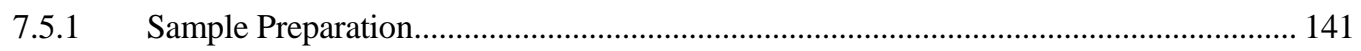

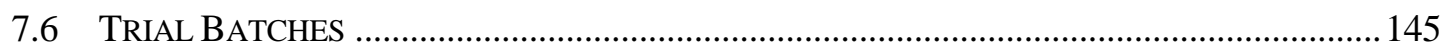

7.6.1 Trial Batches for Conventional Concrete ……………………………....................... 145

7.6.2 Trial Batches for High Strength Concrete ……….................................................... 149

7.7 MAN-MADE CONCRETE SPECIMENS IN A THREE LAYERED APPROACH ...........................151

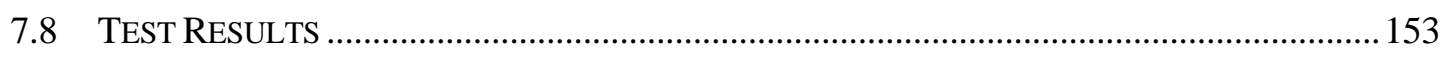

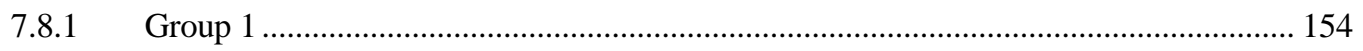

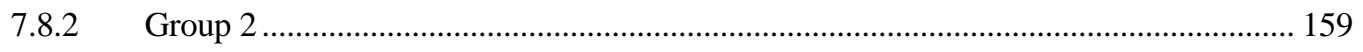

7.8.3 Comparing Acoustic Emission for Groups No. 1 \& No. 2 …....................................... 162

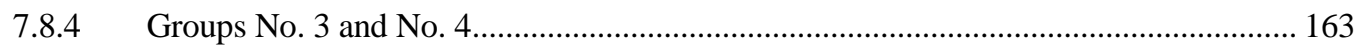

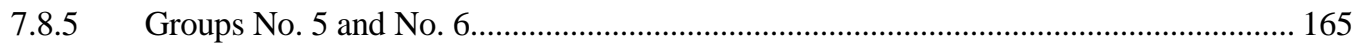

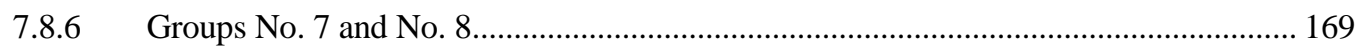

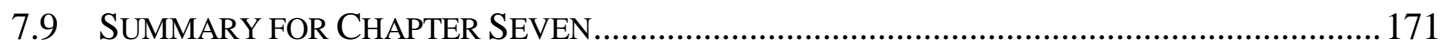

CHAPTER 8: SUMMARY AND CONCLUSIONS .....................................................173

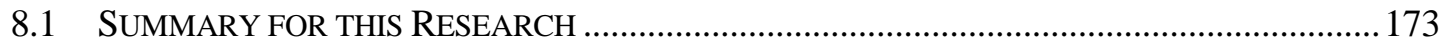

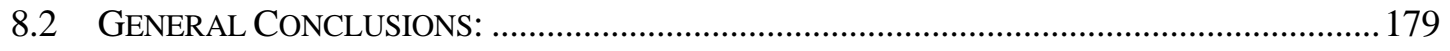

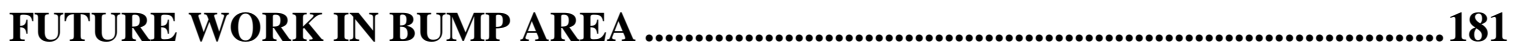

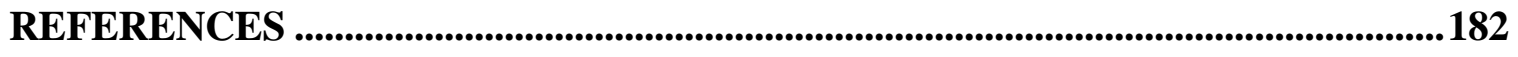




\section{LIST OF FIGURES}

Figure 2-1 A chronological distribution of bumps (Iannacchione and Zelanko,

1995)

Figure 2-2 Reddish staining commonly observed at burst areas (Peng, 2008).............. 19

Figure 2-3 Modified Mohr-Coulomb criterion (Babcock \& Bickel, 1984) .................. 21

Figure 2-4 A conceptual diagram for the critical pillar (DeMarco, 1996) ....................23

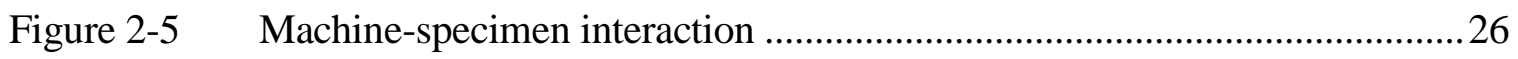

Figure 3-1 A complete stress strain curve of brittle rock (Salamon, 1969) ...................34

Figure 3-2 Stress-strain curves for a) a violent failure and b) a nonviolent failure ........37

Figure 3-3 Variation of the elastic strain energy for modeled coal specimens with different UCS values

Figure 3-4 Coal specimens from the Sunnyside seam, Utah a) without macroscopic

cracks and b) with macroscopic cracks ................................................. 41

Figure 3-5 Cylindrical coal specimens from the Sunnyside seam, Utah after testing ...44

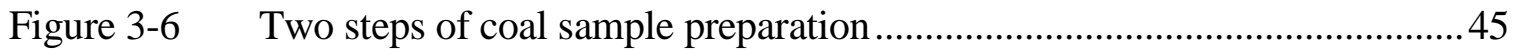

Figure 3-7 Comparing the vertical strength for the Sunnyside seam, Utah coal and

the Sewickley seam, WV coal ...................................................................46

Figure 3-8 Comparing Young's moduli for the Sunnyside seam, Utah coal and the

Sewickley seam, WV coal ...............................................................52

Figure 3-9 Stages of sample preparation for direct shear tests ...................................55

Figure 3-10 Comparing the shear strength for Utah and WV coals ..............................56

Figure 3-11 Elastic and dissipated energy for a) the Sunnyside seam, Utah coal and

b) the Sewickley seam, WV coal ..........................................................58 
Figure 4-1 Variations of released and kinetic energies due to sudden loss of friction .. 65

Figure 4-2 Variation of the vertical stress at different paths due to losing interface

friction

Figure 4-3 The applied load history of modeling from the start to the end of the

analysis

Figure 4-4 Variation of a) elastic strain energy and b) kinetic energy before and after applying the impact load .70

Figure 4-5 Variation of the vertical stress once the impact load was applied at path

3-3.

Figure 5-1 Mohr-Coulomb envelopes for interface friction between coal specimens and loading platens .75

Figure 5-2 Coal specimen loaded by the NIOSH MRS Machine..... .77

Figure 5-3 Coal specimen No. 13 in group 1 of $\mathrm{W} / \mathrm{H}$ ratio $=1$ before and after failure 82

Figure 5-4 Coal specimen No. 17 in group 1 with $\mathrm{W} / \mathrm{H}$ ratio $=3.3$ before and after

failure 83

Figure 5-5 Stress-strain curves for three specimens of different W/H ratios in group

$$
1
$$

Figure 5-6 Stress-strain curve for a specimen No. 30 of $\mathrm{W} / \mathrm{H}$ ratio $=4.7$ in group $2 \ldots 85$

Figure 5-7 Coal specimen No. 30 in group 2 before and after failure .87

Figure 5-8 A typical loading-unloading stress-strain curve for coal specimen in group 3 
Figure 5-9 Variation of (a) the vertical stress and (b) Young's modulus with the same range of $\mathrm{W} / \mathrm{H}$ ratio and different interface frictions.

Figure 5-10 Coal specimen No. 14 in group 4 of $\mathrm{W} / \mathrm{H}$ ratio $=1.2$ before and after

failure .93

Figure 5-11 Coal specimen No. 15. It was loaded to its ultimate strength and then the

load was removed quickly .94

Figure 5-12 Loading-unloading stress-strain curve of specimen No. 15 in group 4 .......95

Figure 5-13 Reloading of coal specimen No. 15 until its failure .95

Figure 5-14 Loading-reloading stress-strain curves of coal specimen No. 15 in group 4 .96

Figure 5-15 Specimen No. 33 in group 5 and its rib and core zones after testing .98

Figure 5-16 Stress-strain curves for a) non-violent failures and b) violent failures for coal specimens in group 5

Figure 5-17 Coal specimen No. 10 before and after failure .103

Figure 5-18 Force-displacement curve for intact coal specimen No. 10 .104

Figure 5-19 Core zone of specimen No. 10 before and after reloading..... .105

Figure 5-20 Force-displacement curves for core zone and intact coal specimen No. 10 106

Figure 6-1 Location of the drilled horizontal holes for a coal specimen of W/H ratio $=3$.

Figure 6-2 Stress strain curves for coal specimens softened by artificial holes: (a)

$\mathrm{W} / \mathrm{H}$ ratio $=3$ and (b) $\mathrm{W} / \mathrm{H}$ ratio $=4$ 116 
Figure 6-3 Coal specimens of $\mathrm{W} / \mathrm{H}$ ratio $=3$ softened by artificial holes before and after failure 118

Figure 6-4 Arrangement of the vertical holes for coal specimens of different W/H ratios .121

Figure 6-5 Stress-strain curves for coal specimens of $\mathrm{W} / \mathrm{H}$ ratio $=4$ with and without core softening

Figure 6-6 Examples of Coal specimens of $\mathrm{W} / \mathrm{H}$ ratio= 4 with core softening before

and after failure 128

Figure 7-1 Probability distribution for UCS test results from 20 coal specimens .138

Figure 7-2 Variation of UCS with changing W/C ratio (Mehta and Monteiro, 2006) 143

Figure 7-3 Curing of concrete specimens 145

Figure 7-4 Stress-strain curves for trial batches No. 1 and No. 2 to simulate medium and strong pillar......

Figure 7-5 concrete specimen in trial batch\#1 before and after failure 149

Figure 7-6 Stress Strain curve for high strength concrete trial mix 151

Figure 7-7 procedures for casting the three-layered concrete specimens 152

Figure 7-8 Stress-strain curves for a) Medium pillar and b) Strong rock

Figure 7-9 Stress-strain curves for group No. 1, strong roof-medium pillar-strong floor 156

Figure 7-10 Concrete specimen in group No. 1 before and after failure 157

Figure 7-11 A screen shot from video at the moment of failure for specimen in group

No. 1 158

Figure 7-12 Stress-strain curves for strong pillar in group No. 2 159 
Figure 7-13 Typical stress-strain curves for specimens in group-2, strong roof-strong

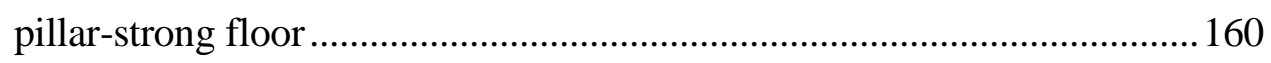

Figure 7-14 A concrete specimen in group No. 2 before and after failure ....................161

Figure 7-15 Peak SPL for specimens in groups No. 1 and No. 2 ................................163

Figure 7-16 Stress-strain curves for specimens in groups No. 3 and No. 4 ................. 164

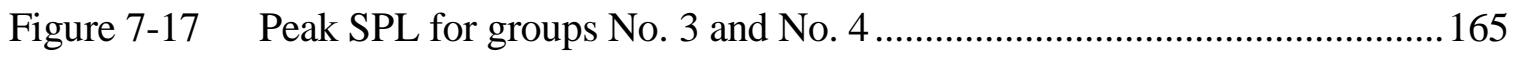

Figure 7-18 Typical stress strain curves for specimens in groups No. 5 and No. 6 ......166

Figure 7-19 A concrete specimen in group No. 5 before and after failure .................... 167

Figure 7-20 A concrete specimen in group No. 6 before and after failure ....................168

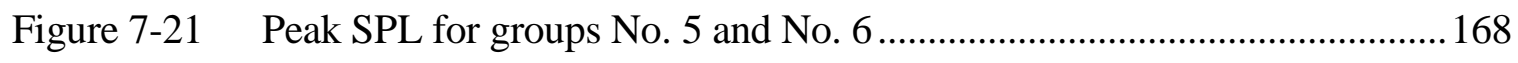

Figure 7-22 Typical stress-strain curves for specimens in groups No. 7 and No. 8 ..... 169

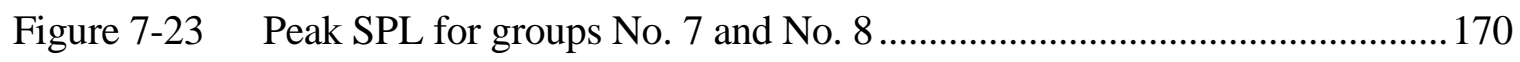

Figure 7-24 Concrete specimens before and after failure for a) group No. 7 and b) group No. 8 


\section{LIST OF TABLES}

Table 3-1 Laboratory test results of coal specimens at different width-to-height

ratios

Table 3-2 Correlation between post-peak slope of stress-strain curve and vertical

strength and $\mathrm{E}$

Table 3-3 Mechanical properties for the modeled coal specimens

Table 3-4 Laboratory test results for cylindrical coal specimen from the Sunnyside

seam, Utah.

Table 3-5 Test results for the Sunnyside seam, Utah coal ........................................48

Table 3-6 Test results for the Sewickley seam, WV coal ..........................................49

Table 3-7 burst susceptibility depending on the burst index .....................................58

Table 3-8 burst susceptibility index for the Sunnyside seam, Utah and Sewickley

seam WV

Table 5-1 Test results for 0.1 and 0.25 interfaces friction........................................ 76

Table 5-2 Mode of failure associated with coal specimens of different $\mathrm{W} / \mathrm{H}$ ratios and end constraint conditions

Table 5-3 Summary of vertical stress test results for the five groups shown in

Table 5-2

Table 6-1 Test results for coal specimens where the ribs were softened by

horizontal holes

Table 6-2 Test results for coal specimens of various width-to-height ratios where

the core was softened by vertical holes 
Table 6-3 Percent reduction in UCS for coal specimens with and without core softening

Table 6-4 Test results for coal specimens of width-to-height ratios equals to 3 and 4 softened completely by water.

Table 7-1 Eight combinations of simulated geological conditions ........................... 135

Table 7-2 UCS test results for 20 cylinder coal specimens of the Sunnyside seam... 136

Table 7-3 S ummary statistics for UCS tests for 20 cylindrical coal specimens from

Utah's Sunnyside seam.

Table 7-4 Classification of UCS for intact rock (Recommendations from ISRM,

based on Brown, 1981)

Table 7-5 Recommended UCS values for coal pillars and rock

Table 7-6 Mixture proportions $\left(\mathrm{kg} / \mathrm{m}^{3}\right)$ for conventional concrete's trial mix

Table 7-7 UCS and tangent modulus for trial batches of medium and strong pillar.. 148

Table 7-8 Trial mix proportion for high strength concrete used to simulate strong

rock 


\section{CHAPTER 1: INTRODUCTION}

\subsection{Background}

Bumps in coal mines have been recognized as a major hazard for many years. Many case histories in mining have described coal pillars or faces of coal failing violently with an accompanying ejection of debris and broken material into the working areas of the mine (Campoli et al., 1987; Osterwald, 1962; Peperakis, 1958; Iannacchione and Tadolini, 2015). These unstable failures have resulted in large losses of life and total collapses of entire mine panels (Chase et al., 1994; Zingano et al., 2004).

A recent documented case of a coal bump occurred on May $12^{\text {th }}, 2014$, in Brody Mine No. 1 in the Eagle Coal Seam, located near Wharton, in Boone County, West Virginia. A large pillar burst occurred while retreat mining was conducted in the No. 6 entry, on the No. 1 Section in the 4 East Mains. Two miners were killed due to the bump (MSHA, 2014). Another case occurred on August $6^{\text {th }}, 2007$, in Crandall Canyon Mine in central Utah, the Sunnyside Coal Seam. A large area of pillars in the South Barrier sections failed violently. Six miners were killed due to that bump. Ten days later during the rescue effort, another bump occurred resulting in three of the rescue workers being fatally injured (Heasley, 2008; Pariseau, 2008).

A coal bumps are not a common phenomenon in mines. Many field investigations on coal mine bumps have been conducted in an attempt to explain why coal pillars fail in such violent fashion. Most of the investigators have considered geological conditions such as strong, immediate roof and floor, strong coal, and large overburden depth as some of the main causative factors leading to the bumps. However, it is not clear in the literature 
whether coal bumps happen because of the local variations in the geologic structures and properties such as sudden changes in the strength of the roof and floor, interface friction, and depth or elevation, or that the coal itself might be the main factor.

This research will focus on exploring the following issues: (1) To what extend coal itself plays a significant role in coal bumps? (2) At what coal specimen's width-to-height $(\mathrm{W} / \mathrm{H})$ ratio and interface friction coefficient would a bump happen? (3) Which part of the coal specimen is the main cause for the violent failure? Is it the ribs or the core of the coal specimen or do both the ribs and the core that play a role in coal bumps? (4) Why are coal bumps generally associated with strong, stiff roof and floor?

\subsection{Statement of the Problem}

Coal mine bumps are sudden, violent bursts of coal from a pillar, or pillars or a block of coal, resulting in a section, the whole pillar, or the solid block of coal being thrown into an open entry, often with shattered coal piling up to the roof line. These bumps are accompanied by audible noises (Peng, 2008). Since understanding the causes of coal mine bumps is essential to creating a safe underground working environment, this phenomenon has motivated many ground control researchers to conduct extensive field investigations throughout the past century. As a result of these investigations, most investigators agreed that there are specific geological conditions that contribute to bumps. These specific conditions include strong and rigid immediate rock strata, strong coal, and significant overburden depth (Campoli et al., 1987; Holland \& Thomas, 1954; Rice, 1935). Iannacchione and Zelanko (1995) stated that among the 95 bump sites reviewed, 86 sites had the presence of strong, massive sandstone. 
Certain geological conditions may be essential in producing bumps, but they may not be completely necessary in order for bumps to occur. For example, bumps have been reported when mining under a weak roof such as siltstone or mudstone (Peng, 2008), at low coal strengths of 1,523-2,140 psi (Brauner, 1994; Holland \& Thomas, 1954), and at a shallow depth of 500 feet (Holland \& Thomas, 1954). Iannacchione and Zelanko (1995) noticed that no single factor was responsible for coal mine bumps; instead, a combination of geology, stress, and mining conditions influenced the likelihood of pillars to bump.

Coal pillar failure, whether it is violent or not, occurs because the load applied to the pillar is beyond the pillar strength. In experiments, the strength of coal specimens is greatly influenced by the friction between specimen and machine platens (Babcock, 1985; Khair, 1968; Meikle, 1965) and specimen size (Daniels \& Moore, 1907; Griffith \& Conner, 1912; Lawall \& Holland, 1937; Pariseau et al., 1977). Since most of the documented cases where coal bumps happened, the roof and the floor are stiff and strong, that might give a hint that these stiff roof and floor act like a stress raiser which concentrates more load on the pillar. More work is needed to explore that area.

It is well known that the size and the shape of the coal sample affect its ultimate strength. Very often coal strength was mentioned in connection with previous researches. However it is not clear whether that strength is for standard cylindrical coal samples of height to diameter ratio $=2.0$ or that strength is for cubical coal samples, or it is for prismatic coal samples of specific width-to-height ratio. This ambiguity about the strength of coal in the previous research prompted me investigating whether or not the strength of coal plays a substantial role in coal mine bumps. 
Many researchers focused only on the size of the pillar to control the frequent occurrence and severity of coal mine bumps. Sometimes, a pillar of a certain size provides excellent ground control in the gateroads, and yet the same pillar in other parts of the same mine performs very poorly. Perhaps the main reason for this variation in pillar performance is that the local variation of the interface friction and stiffness of both the roof and floor in addition to the mechanical properties of coal should be considered in the analysis.

Two questions may be raised now: Does the local variation of the geologic conditions contribute to the occurrence of coal mine bumps irrespective of the strength of the coal itself? And At what geological conditions and width-to-height ratio do coal mine bumps occur? The lack of understanding of the role of: the mechanical properties of coal, the interface friction, the width-to-height ratio, and the strength of immediate roof and floor on the mode of failure behavior of coal specimens prompts me to investigate these factors. It is believed that these factors deserve close attention in explaining the occurrence of coal mine bumps.

\subsection{Research Objectives}

The main objectives of this research can be summarized as follow:

1. Study whether the mechanical properties of coal play a significant role in coal mine bumps.

2. Investigate the effect of specimen width-to-height $(\mathrm{W} / \mathrm{H})$ ratios and interface friction on the potential for violent failure, and determine the critical width-to-height ratios $(\mathrm{W} / \mathrm{H})$ and interface friction where coal bump would happen. 
3. Investigate two of the most common bump mechanisms proposed by Rice (1936) and Holland (1958b) with the aid of Finite Element Modeling (FEM).

4. Determine which part of the coal specimen is responsible for coal bumps, i.e., does the core zone or the rib zone or both contribute to bumps.

5. Investigate the influence of the roof and floor strength on the potential for violent failure.

\subsection{Research Methodology}

To achieve the first objective in this research, the role of the mechanical properties of coal in bumps, laboratory tests were conducted on a non bump-prone coal ( the Sewickley seam, WV coal) and a bump-prone coal (the Sunnyside seam, Utah coal). The main objective of these tests is to investigate whether or not coal itself plays a significant role in coal mine bumps. The mechanical properties of coal such as, UCS, Young's modulus, cohesion \& friction angle, and burst index will be compared for these two coals. The results of that comparison will indicate whether or not coal itself is bump prone.

To achieve the second objective in this research, the role of interface friction and width-to-height ratio on the mode of failure behavior of coal specimens, coal specimens of different $\mathrm{W} / \mathrm{H}$ ratio and interface frictions were prepared and tested. The $\mathrm{W} / \mathrm{H}$ ratio ranges from approximately 1 to 10 , while the interface friction is 0.10 and 0.25 . The NIOSH MRS Machine was used to conduct uniaxial compression test for coal specimens of different $\mathrm{W} / \mathrm{H}$ ratios and interface frictions. (The reason for using the NIOSH MRS Machine is that the strength of many samples, in particular those with high $\mathrm{W} / \mathrm{H}$ ratio, 
exceeds the ultimate strength of the WVU MTS machine). The mode of failure has been examined for these coal specimens after UCS testing to determine the critical $\mathrm{W} / \mathrm{H}$ ratio where the mode of failure changes from violent to non-violent. Also, the core zones of the failed coal specimens have been examined to determine whether they are elastic or not. Investigating the core zone is important to accept or refuse the author's assumption that "the sudden failure of the core zone releases the energy stored in it which would cause coal bumps".

To achieve the third objective, investigating two of the most common bump mechanics, Finite Element Models were conducted for simulated coal specimens to examine how an instantaneous loss of constraint or a sudden impact load causes violent failure. The variation of the energy released and the kinetic energy were examined.

To achieve the fourth objective, determining which part of the coal specimens is responsible for bumps, laboratory tests were conducted on coal samples where different zones of the coal sample were softened.

To achieve the last objective of this research, examining the effect of roof and floor strength on the potential for violent failure, man-made concrete specimens were cast to simulate different roof/pillar/floor strength combinations. The concrete specimens are divided into three layers, the top layer simulates the roof, and the middle layer simulates a pillar while the bottom layer simulates the floor. The strength of the three layers was predetermined and well known before running the tests.

\subsection{Test Conditions and Assumptions}

The rate of loading for all tests conducted under load control is between 315 and $320 \mathrm{lb} / \mathrm{sec}$. For the laboratory tests, the failure of coal specimens is considered to be non- 
violent when the following conditions are encountered: The coal specimen does not lose its strength suddenly after reaching the ultimate strength and the magnitude of noises of cracking during testing is not high. Conversely, the failure of a coal specimen is considered violent, when there is a sudden loss of strength and the magnitude of noises of cracking is high.

Since water content would affect the mechanical properties of coal specimens, in particular the strength, all coal specimens in this research were air dried before testing them (except in chapter six, some specimens are tested wet to study the effectiveness of water on mitigating the violent failure of coal specimens).

Whenever a specific interface friction is proposed/assumed on a surface of a coal specimen, it is assumed that this coefficient of friction is the same everywhere on that surface.

\subsection{Extending the Laboratory Work on Coal Specimens to Coal Pillars}

In the author's point of view, although most of the work done in this dissertation was laboratory work conducted on coal specimens, the results and the general conclusions drawn from this work can be applied to coal pillars having the same conditions for the following reasons:

1. The tested coal specimens are considered to be small-scale model of coal pillars having the same material, shape and aspect ratio, i.e., both have the same width-toheight ratio.

2. The tested coal specimens and the coal pillars have the same boundary conditions and constraints, i.e., both have the same interface friction. 
3. The tested coal specimens and coal pillars have gone through the same geological cycle and tectonic forces.

Therefore, these three previous factors provide some evidence to support my claim that the findings and the main conclusions drawn from the experimental work conducted on coal specimens can be extended to coal pillars. However, it is anticipated that a proper application of these findings and conclusions needs further modifications due to that huge differences in the size (the size effect) between coal pillars and coal specimens. 


\section{CHAPTER 2: LITERATURE REVIEW}

\subsection{Introduction}

Violent failures of coal pillars occurred in a wide variety of mining environments and mine layouts. The literature review in chapter two covers the previous studies on the influence of interface friction, $\mathrm{W} / \mathrm{H}$ ratio, the mechanical properties of coal material and local mine stiffness on the potential for violent failure. Moreover, different theories proposed to explain and mitigate coal bumps.

\subsection{Common Terms}

A brief description for some terminologies found in the literature and used in this dissertation is provided below. Most of these terms commonly found in the studies of failures and, more specifically, coal mine bumps and unstable failures.

$>$ Coal bumps are a form of unstable failure in which a violent ejection of coal pillar(s) or a block of coal into the open entry, it may result in injury to mine personnel and/or significant damage to equipment or underground workings. A bump may be accompanied by air blast and a cloud of pulverized coal dust that might destroy the ventilation system. If ejection of coal is due to the release of a large amount of highly pressurized gas, especially methane, it is called a gas outburst.

Rockbursts are similar to coal bumps, but occur in hard rock mines.

The rib refers to the unconfined side of a pillar which is prone to ejections due to a lack of confinement. 
Abutment zone is the zone of high stress which surrounds every opening or hole in the ground. Abutment zones also occur around faults and other irregularities in the earth's crust.

$>$ Outburst is defined as a violent, simultaneous release of gas(es) and comminuted rock material into a working face.

The unintended collapse of large portions of the roof associated with pillar bumps may lead to large air blasts in which a sudden increase in air pressures may cause injury and death along with damage to ventilation equipment.

$>$ Retreat mining is a form of secondary mining in room-and-pillar layouts in which primary support pillars are mined and the roof is allowed to cave behind the active mining area.

Fracture is the failure process by which new surfaces in form of cracks are formed in the rock material or existing crack surfaces are extended.

$>$ Stable fracture propagation is the failure process of fracture propagation in which the crack extension is a function of the loading and can be controlled accordingly. Note that stable fracture propagation results in a controllable failure, which is a non-violent failure.

Unstable fracture propagation is the failure process of fracture propagation in which the crack extension is also governed by factors other than loading, thus becomes uncontrollable. Note that unstable fracture propagation results in an uncontrollable failure, which is a violent failure.

Strength failure is the failure process by which a material changes from a state in which its load-bearing capacity is either constant or increases with 
increasing deformation to a state in which its load-bearing capacity is decreased or has been vanished. Sudden loss of strength was used as one of the criteria used to discriminate between violent and non-violent failures.

Brittle failure is defined as failure that exhibits no or little permanent (plastic) deformation. The opposite term is ductile failure which is preceded by appreciable plastic deformation.

The elastic strain energy is the energy stored in the material upon deformation, the strain energy in the form of elastic deformation is mostly recoverable in the form of mechanical work. The elastic energy is a potential energy since it is stored in the material. If the material failed or the applied load was removed, the elastic strain energy would be converted to another form of energy such as a kinetic energy. It is believed that the occurrence of coal bumps requires the release of elastic strain energy and kinetic energy.

The kinetic energy of an object is the energy that it possesses due to its motion.

Pitch of noise is the synonym for an acoustic emission, where cracks initiation and propagation within a material are associated with audible noise. This noise occurs because of the transient elastic waves generated by the rapid release of energy within a material. In chapter seven, sound pressure equipment was used to discriminate between the violent and non-violent failures. However, the author used a qualitative approach depending on his experience in the remaining chapters. 
Loud or high pitch noise occurs when the coal specimens fail violently where the sound pressure level associated with their failure is high enough to hurt the ears of the person conducting the tests.

$>$ UCS is the unconfined compressive strength used only when the height-towidth ratio of the tested specimens is close to two, while the term "vertical strength" is used rather than the "UCS" when the height-to-width ratio is less than two.

\subsection{Coal Mine Bumps}

Coal mine bumps have presented serious mining problems in the United States throughout the $20^{\text {th }}$ century. Fatalities and injuries have resulted when these destructive events occurred at the working faces of the mines. A chronological distribution of bump events included in USBM coal bump data base from 1930 to 1995 is shown in Figure 2-1, which indicates that bump continued to occur at an alarming rate. A total of 78 fatalities and 158 injuries were identified. In addition to the statistics shown in Figure 2-1, coal bumps occurred recently, for instance on August $6^{\text {th }}, 2007$, in Crandall Canyon Mine in central Utah, Sunnyside Coal Seam, six miners were killed. Ten days later during the rescue effort, another bump occurred resulted in killing three of the rescue workers. On May $12^{\text {th }}, 2014$, in Brody Mine No. 1 in the Eagle Coal Seam, two miners were killed due to the bump (MSHA, 2014). 


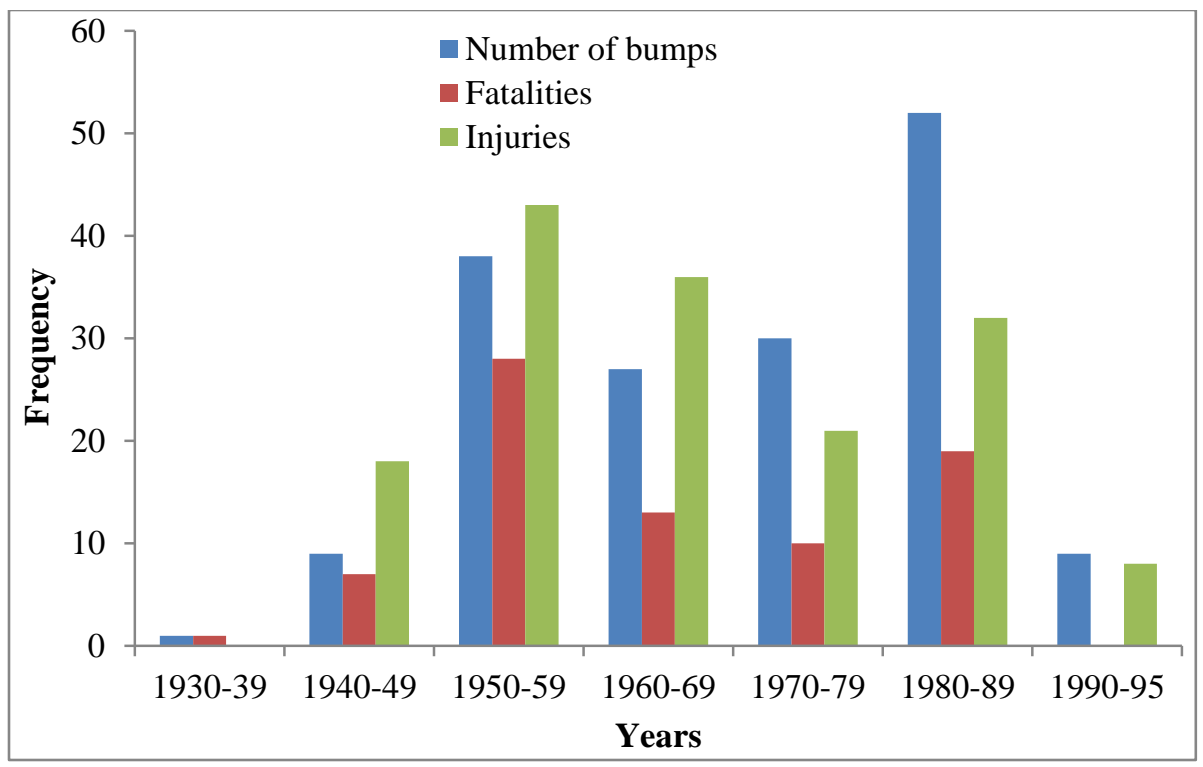

Figure 2-1 A chronological distribution of bumps (Iannacchione and Zelanko, 1995)

From an energy prospect, coal bumps can be characterized as the unstable release of energy associated with the post-peak stage of pillar loading that occurs with the progression of mining. Unstable releases of energy occur when the coal is unable to absorb the energy that is released by the surrounding rock mass during the failure process. At any stage of mining, the mining geometry, the overburden stress, the elastic properties of rock mass, and the stress-strain characteristics of the seam material govern the amount of excess energy so released (Crouch and Fairhurst, 1973).

Coal bump can be classified as either "pressure bumps" or "shock bumps" (Rice, 1935). A pressure bump is due to the violent failure of a pillar when its maximum loadbearing capacity is reached. A shock bump is due to the breakage of a thick, rigid stratum at a distance above the coal seam causing a hammer-like blow or a shock wave being transmitted to the coal pillars. If the pillar is highly stressed, it could fail suddenly. The essential difference between these two types of coal mine bumps is a matter of loading rate: 
in the case of a pressure bump, the rate of loading is considered to be quasi-static, while it is dynamic for a shock bump. In practice, it is difficult to determine which type of coal bump has occurred. Many investigators explain the coal bump mechanism as more closely related to a pressure bump mechanism rather than a shock bump mechanism (Morsy, 2003).

Several field investigations have been conducted since 1935 to study the causes of bumps. Investigators have agreed generally to assign a definite set of natural conditions of geology as the causative factors leading to coal mine bumps, though these geological factors do not completely explain why bumps occur.

\subsection{Coal Pillar Failures}

A coal pillar is designed to support the overburden and protect the adjacent entries/crosscuts. Failure occurs when the load is applied to a pillar beyond its ultimate strength. Therefore, failure of a coal pillar means the inability of the pillar to serve its designated functions (Peng, 2008).

Coal pillars may fail gradually or violent depending on the geological condition and the mechanical properties of coal (Haramy \& McDonnell, 1988; Holland, 1958b; Holland \& Thomas, 1954). When coal pillars fail gradually, they deform axially and laterally in response to the load applied to them during their service life. The deformation may be noticeable. Examples of gradual failure of coal pillars include rib spalling or rib sloughing, which is mainly due to the lateral expansion of pillars and orientation of entries/crosscuts with respect to the cleat system (Peng, 2008). Violent pillar failures are more instantaneous in terms of stress changes (Maleki, 1995) and are usually unpredictable and without any preliminary warning (Rice, 1935). 


\subsection{Geological Conditions and Coal Mine Bumps}

Field investigation of the coal mine bump phenomenon began with Rice (1935), who examined various coal mines of the Cumberland field in Harlan County, Kentucky, and Wise County, Virginia. This investigation was triggered by a series of coal mine bumps that caused many deaths and injuries and that had been increasing in frequency during the preceding few years to Rice's study.

Favorable conditions contributing to coal mine bumps - These conditions can be summarized as follows:

Large mining depth - in most bump cases, the onset of bump problems occurred around a depth of $1,500 \mathrm{ft}$ or more, but in some cases bumps occurred at depths as shallow as $750 \mathrm{ft}$ (DeMarco et al., 1995).

$>$ A structurally strong coal - several researchers believed that a strong, brittle coal with a high compressive strength is necessary for bumps. However, Babcock (1985) analyzed this concept in the laboratory and concluded that coal bumps could occur regardless of the strength of coal. He hypothesized that pillar bumps are caused by the sudden release of end constraint at the roof and floor.

Strong overlying rock stratum or strata within a distance of 10 to 15 times the thickness of the coal seam (Rice, 1935). One of the leading contributors to coal bumps in many cases is a massive sandstone unit that commonly makes up the main roof. This main roof is able to span the gobs of several panels before they fail and reach a state of maximum subsidence (DeMarco et al. 
1995). The sudden failure of these units is also a contributing factor in the initiation of coal bumps (Maleki, 1995).

A strong floor that is not subjected to heaving. If the floor is soft and thick, a pillar is likely to punch into the floor and the elastic energy stored in the pillar will be dissipated (Peng, 2008). Conversely, if the floor is strong and thick, it will help the pillar to store sufficient energy to fail violently.

It is expected that strong roof \& floor and the interface friction between the pillar and the roof and the floor provide the coal pillar with supplemental strength. Therefore, the strength of a coal pillar consists of two parts: The first part is the inherent strength due to the mechanical properties of the coal. The second part is supplemental strength due to the constraint derived from the surrounding environments.

Rice (1935) mentioned that the natural condition is one of the key factors associated with coal mine bumps. According to Rice, natural conditions were thought to be strong and rigid rock strata, structurally strong coal, and overburden depth greater than 1,000 feet deep or even 500 feet deep when mining under a steeply rising area. These conditions were unavoidable because they exist naturally in many mines. Campoli et al. (1987) reviewed five cases of coal mine bump problems in the eastern US and concluded that thick overburden and extremely rigid strata immediately above and below the mined coal beds were conducive to the bumps.

Holland and Thomas (1954) analyzed 117 occurrences of bumps in US coal mines and found that bumps had been reported when the depth of cover was only 500 feet and when strong immediate overlying and underlying strata were present (usually a massive sandstone or a conglomerate). 
Faulty mining methods- Coal mine bumps occurred in both longwall mining and room and pillar mining methods. In longwall mining, coal bumps generally occurred in the tailgate chain pillars due to multiple side abutment loads created by the previous gobs. Bumps also occur at the longwall face near the tailgate end and the panel ribs of the tailgate within 60-100 ft outby the tailgate T-junction (Peng, 2008).

In room and pillar mining, the major cause of coal mine bumps is the retreat pillar mining followed by the barrier splitting. Depending on the cutting method and the cutting sequence, the pillar line varies from time to time. Consequently, certain pillars may receive higher loads transferred from others that are being cut. This additional load may be enough to create bumps of those pillars (Peng, 2008).

According to Rice (1935), faulty mining methods associated with coal mine bumps are: making pillars too small, leaving projecting pillars in the line of pillar withdrawal, narrowing to points by diagonally slicing the inby ends of pillars, and pulling pillars in separate panels rather than taking out room entry pillars on long retreat lines. Holland and Thomas (1954) discovered that about 67.6 percent of the bumps took place in areas associated with pillar-line points during pillar-recovery operations or adjacent to an area where pillars were being or had been extracted. Thus, excessive loads resulting from the superimposition of abutment areas on pillars were likely contributing to bumps.

Multiple seam mining, if not properly planned, is known to cause seam interaction resulting in areas of high stress concentration, which might cause coal mine bumps (Peng, 2008).

Presence of structural anomalies- Faults and sandstone channels are the most common structural anomalies associated with coal mine bumps. Sandstone channels are 
stress concentrating structures that are directly related to bumping along longwall panels. The massive nature of these units is the major factor affecting bump initiation (Peng, 2008). Iannacchione and Zelanko (1994) reviewed the lithologic description of mine roofs from 95 bump sites. They found that 86 sites had the presence of sandstone and as many as 30 sites had shale, sandy shale, siltstone, or mudstone sandwiched between the sandstone layers in varying thicknesses. They concluded that no single factor was responsible for coal mine bumps. Instead, bumps occurred as a result of complex interaction between geology, stress, and mining conditions.

\subsection{An Interface Friction and Coal Mine Bumps}

Laboratory experiments have shown that coal specimen strength greatly depends on interface friction between coal specimens and machine platens (Babcock, 1985; Khair, 1968; Meikle \& Holland, 1965), and specimen size (Daniels \& Moore, 1907; Griffith \& Conner, 1912; Lawall \& Holland, 1937; Pariseau, et al., 1977). However, little attention has been given to the role of interface friction and width-to-height ratio with regard to coal mine bumps although their contributions have been cited as a possible contributing factor for bumps.

Holland (1958b) and Meikle \& Holland (1965) were probably the first to introduce a hypothetical effect of friction in generating pillar bursts. This was explained for the first time by Holland (1958b), while Meikle and Holland (1965) merely investigated the effect of friction on the strength of model coal pillars. Nonetheless, both came up with a hypothetical conclusion about the role of sudden loss of friction in generating a pillar burst.

Holland (1958b) stated that friction between the coal pillar and the floor and top rock develops frictional forces that resist the expansion of the pillar induced by the 
overburden load. This mechanism creates a triaxial state of stress which results in strengthening the coal pillar as the pillar is allowed to absorb more axial stress. In addition, the frictional forces will provide constraint to the central part of the pillar and increase with pillar size. When this constraint is suddenly lost by means of some sort of mechanism, stresses that have been highly developed from the triaxial condition are suddenly released and allow a great expansion of the coal pillar in a severe manner, permitting the violent failure of pillar(s) to occur.

This concept was strengthened by the observation that brown stains (commonly known as red-dust) on the roof have been commonly seen at the burst areas. These stains were believed to be an indication of relative motion between the burst-coal pillar and adjacent strata when the friction is being overcome (Holland, 1958b). Later on, this finding was confirmed by other researchers who found the same feature at many bump locations. Several descriptive terms had been used, such as "dusting of red coal" (Iannachione \& Zelanko, 1994), "red dust” (Maleki, 1995), "reddish brown coal” (Newman, 2002), and "reddish tint" (Peng, 2007). This feature can be seen in Figure 2-2.

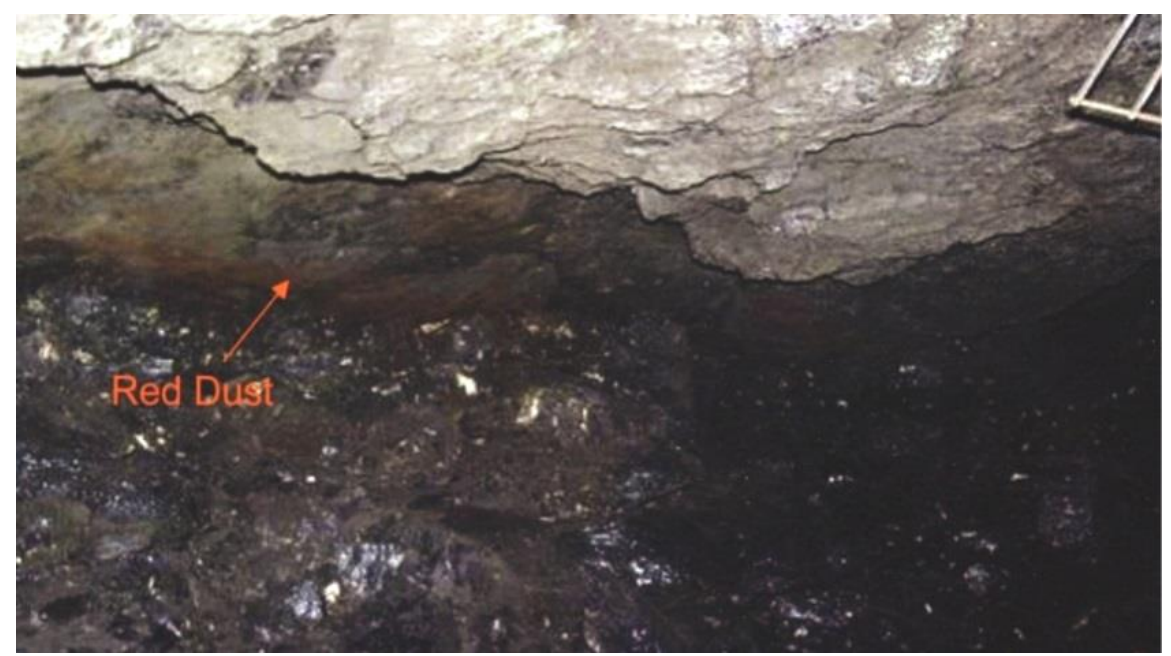

Figure 2-2 Reddish staining commonly observed at burst areas (Peng, 2008) 
Holland (1958b) theorized that interface friction between a coal pillar and adjacent strata may contribute to the violent failure of coal pillars. However, since then, a few field investigations or laboratory experiments have been done to prove this concept. Babcock and Bickel (1984) seemed to be the only researchers to extend Holland's hypotheses. They conducted laboratory experiments on coal samples obtained from 15 mines in 11 coal seams in 6 states. The coal specimens were square in shape, 2.13 in wide and 0.25 in thick $(\mathrm{W} / \mathrm{H}=8.5)$. They predicted that the burst would happen when the constraint was lost.

According to Babcock and Bickel (1984), most coals can be made to burst if the appropriate stress and constraint are present. They provided a simple explanation for the failure mechanism by a simple modification of the Mohr-Coulomb criterion, where a rapid decrease in the confinement (note the confining pressure for the stress-circle \#1 and stresscircle \#4 shown in Figure 2-3) results in a dynamic increase of the Mohr circle as shown in Figure 2-3. It is obvious that once the circle exceeds the static failure envelope, the burst will occur. 


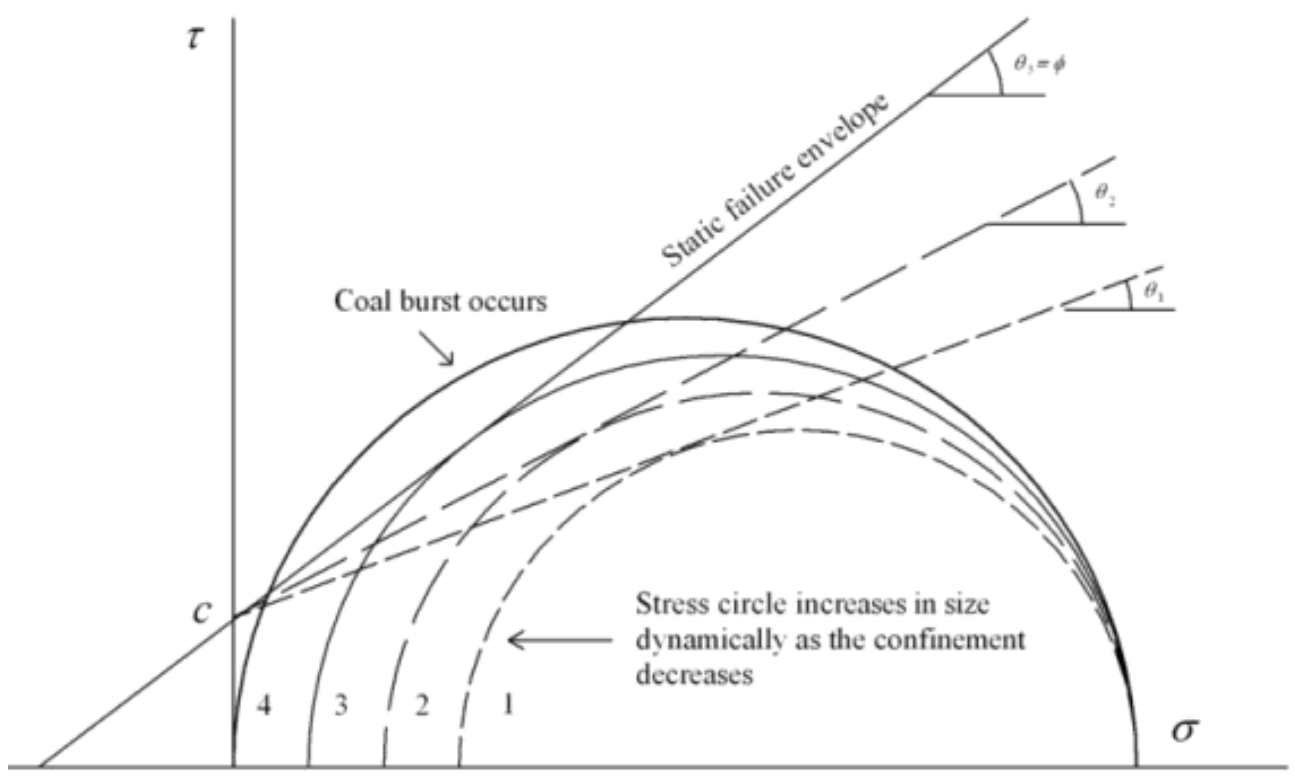

Figure 2-3 Modified Mohr-Coulomb criterion (Babcock \& Bickel, 1984)

Babcock and Bickel (1984) found that the coal can be made to burst if the friction that provides constraint to the coal specimens is suddenly lost. This finding was one step further than that of Holland (1958b), who had first proposed the concept. However, this finding was still limited to one interface friction (between coal specimen and the segmented platen) and one $\mathrm{W} / \mathrm{H}$ ratio of 8.5 . This limitation motivated this research to expand the interface friction value and use a diverse $\mathrm{W} / \mathrm{H}$ ratio of coal specimens.

Prassetyo et al., (2011) studied the effect of interface friction on the potential for violent failure of coal specimens having different $\mathrm{W} / \mathrm{H}$ ratio. He used sound pressure level to differentiate between violent and non-violent failures. He found that the potential for violent failure increases with increased interface friction. 


\subsection{The Width-to-Height Ratio and Coal Mine Bumps}

According to Ozbay (1989), pillars with width-to-height ratios of 4 to 5 can promote bumps. He also concluded that a pillar with a width-to-height ratio greater than 5 could not bump because its post-peak stiffness should be zero or more. However, it has been reported that even the pillars with width-to-height ratios greater than 8 have experienced bump failures (Babcock and Bickel, 1984).

The nature of the pillar collapse, i.e., controllable or uncontrollable, is governed by the stiffness of the surrounding rock and the width-to-height ratio of the pillar (Mark 1999; Salamon 1970; Zingano, 2004). Violent failure for pillar occurs when the W/H ratio is less than 3 and the safety factor is less than 1.5 (Mark, 1999; Zingano, 2004).

Holland (1958b) found that the pillar fails violently when the width/height ratio is low and tend to fail gradually when the width/height ratio is larger, and that the width-toheight ratio at which coal's transition from violent to gradual failure increases with coal strength.

The critical pillar concept has been introduced by DeMarco (1996). A "critical pillar" is simply defined as one that is too large to yield either nonviolently or to yield before the roof and floor sustain permanent damages and is too small to support the full abutment loads. The relationship between critical-pillar designs, and yield and abutment pillar designs is presented in Figure 2-4. The horizontal axis represents the minimum performance separating stable from unstable gateroad configurations. A pillar design whose performance falls above the horizontal axis is considered stable, while a design whose performance falls below the horizontal axis is considered unstable. An important aspect of 
the critical pillar concept is the abrupt transition between the fully successful yielding gateroad and the worst possible critical pillar conditions.

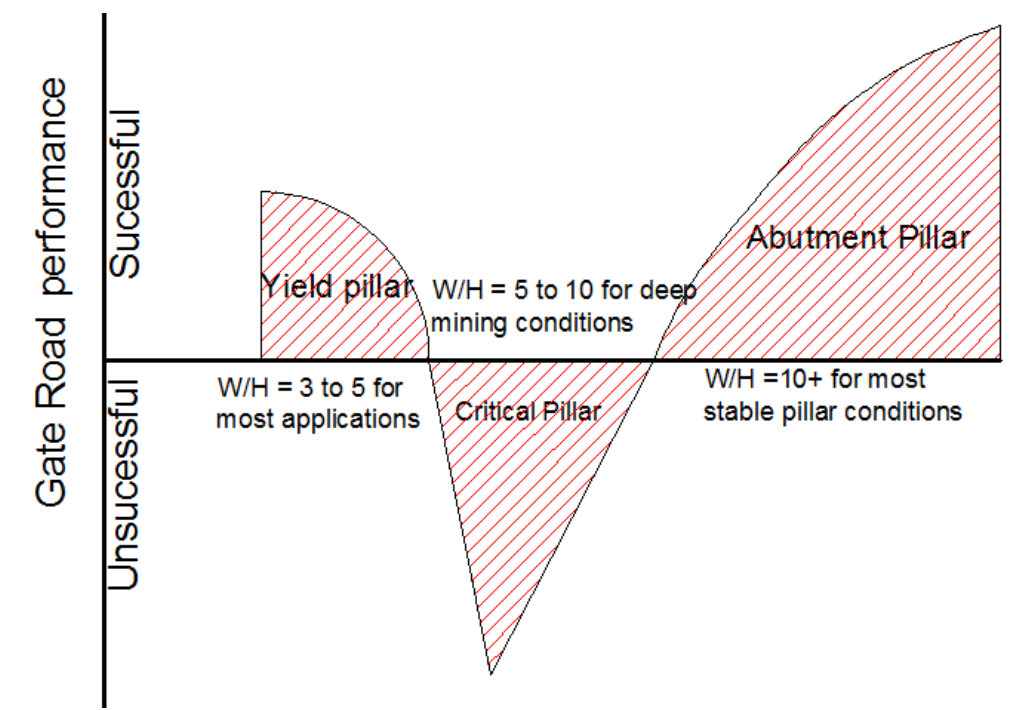

Figure 2-4 A conceptual diagram for the critical pillar (DeMarco, 1996)

Figure 2-4 should not be used to suggest that pillar width alone can completely determine whether a pillar design falls in the critical range. Other mining parameters, such as the overburden depth, and the geo-mechanical properties of the roof and floor strata, can have profound effects on the final disposition of a specific geometry. In practice in order to determine the width of critical pillar, a tapering pillar experiment has been conducted (Serata, 1985).

Garvey (2013) used FLAC ${ }^{3 D}$ to calculate the excess energy from the unstable failure. A three-dimensional pillar model was developed to represent the tributary area loading of an infinite room-and-pillar layout. Garvey found that the smallest pillar geometries released the highest magnitudes of excess energy. This conclusion is consistent with the massive pillar collapse scenario. Zelanko and Heasley (1995) suggested that properly sized gateroad pillars can mitigate tailgate face bumps in many situations by limiting the transfer of abutment stresses to the longwall face. 
Prassetyo (2010) studied the influence of width-to-height ratio on the potential for violent failure. Prassetyo used Sound Pressure Level (SPL) as a criterion for the bump. He found that the SPL decreased with increasing the width-to-height ratio of the sample, which means that the potential for violent failure decreases with increasing the width-to-height ratio. When Prassetyo changed the $\mathrm{W} / \mathrm{H}$ ratio of the coal samples, he fixed the width to 3.0 in and changed the height of the coal samples. Consequently, as the width-to-height ratio increases, the coal samples become thinner, in particular, those with large W/H ratios such as, $10 \& 12$ and 16 . The author have some doubts about testing very thin coal samples, because they will experience big constraint from both the upper and the lower platens. Prassetyo in his study could not determine specific $\mathrm{W} / \mathrm{H}$ ratios and interface frictions where the mode of failure changes from violent to non-violent failure.

\subsection{Strength of Coal and Coal Mine Bumps}

The UCS of all coal seams where bumps caused fatalities and serious injuries ranged from 1,100 to 5,862 psi. (Peng, 2008). In Eastern Kentucky, the Darby seam has had an extensive history of coal bumps and pillar bursts. The UCS of the Darby seam ranges from 3,000 to 5,000 psi with an average strength of 3,980 psi (Iannacchione et al., 2008). Mine 3 operating in the Pocahontas \#3 coalbed near Keen Mountain, Buchannan County, VA experienced bumps (Campoli et al., 1987) yet the strength of the Pocahontas \#3 coalseam is only 1,798 psi (Peng 2008). Brauner (1994) noticed that bursts occurred in almost all types of hard coals. Barron (1990) found that the coal samples of 3000 psi fail non-violently when loaded between Teflon platens, but burst violently when loaded between stiff glass platens. 
Chen et al., (1999) found that for the D seam in Utah, violent failures occurred when the specimens reached a UCS greater than 4,000 psi and non-violent failure modes were observed when the strength was less than 4,000 psi. However, Chen did not mention neither the shape nor the dimensions of the tested laboratory specimens.

In contrast, Lawson et al. (2015) focused on the mineral composition of coal rather than the mechanical properties. She found a very strong correlation between low organic sulfur content, high volatile matter, and positive-bump history. The number of bumppositive samples increases with decreasing sulfur and increasing volatile matter.

\subsection{The Local Mine Stiffness and Coal Mine Bumps}

The stability of a laboratory compression test of a brittle rock specimen depends on the stiffness of the loading machine and the slope of the complete force-displacement relation of the specimen (Salamon, 1969). Figure 2-5 illustrates the interaction between a specimen and a conventional testing machine. The specimen and the machine are regarded as springs loaded in parallel. The machine is represented by a linear elastic spring of constant stiffness, $\mathbf{k}$, and the specimen by a non-linear spring of varying stiffness, $\boldsymbol{\lambda}$. As the specimen compressed, the machine spring extends (Brady and Brown, 1999). 


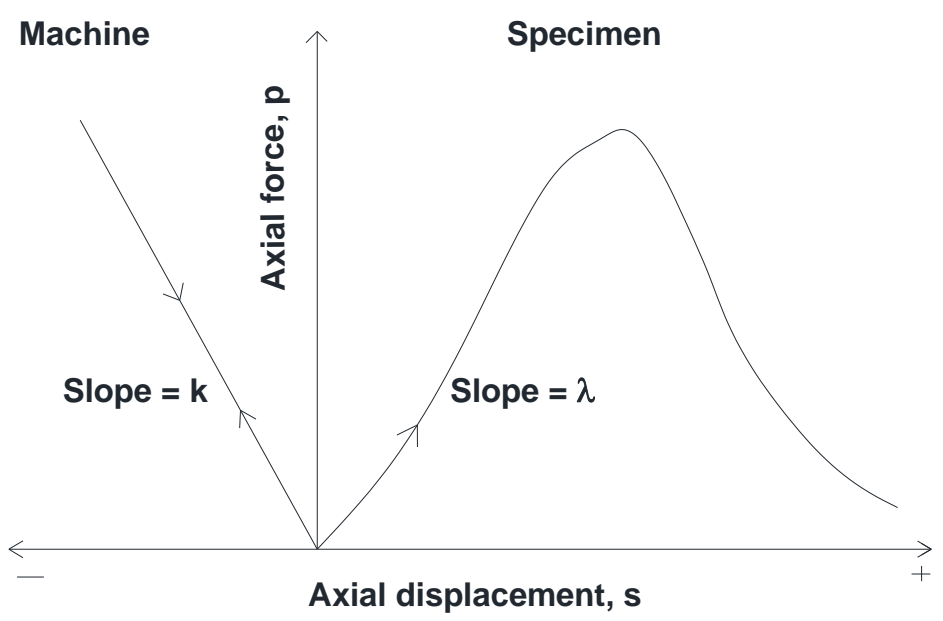

Figure 2-5 Machine-specimen interaction

By generalizing the simple failure mechanism of the laboratory test to the mining situation, the pillar will be stable if equation 2.1 is satisfied; no matter how much displacement the pillar has experienced (Pen and Barron, 1994).

$$
k_{1 i}+\lambda_{i}>0
$$

Where: $\mathrm{k}_{1 \mathrm{i}}$ is the local mine stiffness with respect to the $\mathrm{i}$-th pillar, $\lambda_{\mathrm{i}}$ is the stiffness of the $\mathrm{i}$-th pillar.

The local mine stiffness is determined from the perturbation method proposed by Starfield and Wawersik. In that method, a pillar is substituted by an imaginary hydraulic jack. A small uniformly distributed load increment is imposed at the interface of the roof and the jack which are initially in equilibrium and the accompanying displacement on the jack is determined. The ratio of the force increment to the resulting displacement is defined as the local mine stiffness for that particular pillar position. The most reliable method to determine the stiffness of the pillar would be to directly load the pillar to failure in the field and obtain the load-displacement curve for the pillar. 
The next chapter focuses on answering the following question: To what extent do the mechanical properties of the coal have an effect on the potential for violent failure. To answer that question, a comparison has been conducted between two kinds of coal, one of them is from a bump prone-mine while the other is from a non bump-prone mine. The main reason for studying the role of the mechanical properties of coal on the potential for violent failure is that, there is a debate in the literature about whether the coal strength plays a significant role in bump occurrence or not. 


\section{CHAPTER 3: THE ROLE OF THE MECHANICAL PROPERTIES OF COAL IN BUMPS}

\subsection{Introduction}

Understanding the causes of a coal mine bump is essential to create a safe underground working environment. This phenomenon has motivated many ground control researchers to conduct extensive field investigations throughout the past century. Most investigators (Holland, 1958b; Iannacchione and Zelanko, 1994; Campoli et al., 1987) agreed that the geological conditions leading to coal bumps include great overburden depth and strong and stiff overlying stratum. However, there are questions about whether the coal strength itself plays a significant role in bumps or not. On the one hand, Rice (1935)

mentioned that a structurally strong coal is among the necessary conditions that may contribute to bumps. Brauner (1994) noticed that bursts occurred in almost all types of hard coals. On the other hand, Mine 3 operating in the Pocahontas \#3 coalbed near Keen Mountain, Buchannan County, VA experienced bumps (Campoli et al., 1987) where the strength of Pocahontas \#3 coal seam is 1,798 psi that is low.

The influence of the mechanical properties of coal on the potential for violent failure does not receive much attention in the literature. Moreover, the effect of coal strength on bumps is not clear in the literature. Possibly because the shape and size of the tested coal samples were not often mentioned explicitly in the literature. It is well known that the size and the shape of the coal sample affect its ultimate strength. In the literature, very often coal strength is mentioned without mentioning the shape and/or the size of the coal samples. Hence it is not fair to compare the strength of different kinds of coal where 
the shape and the size of the samples are different or unknown. So this ambiguity about the strength of coal prompted me to investigate whether or not the mechanical properties of coal play a substantial role in coal mine bumps.

The main objective of this chapter is to answer the following question: Do mechanical properties of coal have a substantial effect on the potential for violent failure? One approach to answer this question is to compare the mechanical properties of two kinds of coals from bump-prone (the Sunnyside seam, Utah) and non bump-prone (the Sewickley seam, WV). This comparison includes uniaxial compressive strength, modulus of elasticity, shear strength (cohesion and friction angle), and the burst index.

It is believed that such a detailed laboratory testing plan will help determine whether coal itself is one of the main causative factors for coal bumps or not. If the coal itself is not among the main factors contributing to bump, other factors such as the local variation of the geological conditions need further investigation. Note that the term "vertical strength" will be used rather than the "UCS" when the width-to-height ratio of the tested samples is higher than 0.5 .

\subsection{The Effect of UCS and Young's Modulus on the Potential for Violent Failure}

The unconfined compressive strength, UCS, (or the "vertical strength" when the width-to-height ratio is higher than 0.5 ), the modulus of elasticity and the slope of the post-peak stress-strain are shown in Table 3-1 for coal specimens of different W/H ratios. The slope of the post-peak was measured clockwise from the horizontal of the stressstrain curves. The coal specimens have square cross-sectional areas. Their height was kept constant at $1 \mathrm{in}$, while the width-to-height ratio of the test specimens ranged from 2 
to $4 \mathrm{in}$. All tests were performed under a constant strain rate of $10^{-4} \mathrm{in} / \mathrm{sec}$. Coal specimens of $\mathrm{W} / \mathrm{H}$ ratios greater than 4 could not be tested because of the limiting load capacity of the WVU MTS machine employed for this research. 
Table 3-1 Laboratory test results of coal specimens at different width-to-height ratios

\begin{tabular}{|c|c|c|c|c|c|c|c|c|}
\hline $\begin{array}{l}\text { W/H } \\
\text { ratio }\end{array}$ & $\begin{array}{c}\text { Specimen } \\
\#\end{array}$ & $\begin{array}{c}\text { Vertical } \\
\text { strength, } \\
\text { psi }\end{array}$ & $\begin{array}{c}\text { Vertical } \\
\text { strength }_{\text {Avg., }} \\
\text { psi }\end{array}$ & $\begin{array}{c}\text { Vertical } \\
\text { strength }_{\text {std. }} \\
\text { psi }\end{array}$ & $\begin{array}{l}\text { E, } \\
\text { psi }\end{array}$ & $\begin{array}{c}\mathbf{E}_{\mathrm{Avg}} \\
\mathrm{psi}\end{array}$ & $\begin{array}{r}\mathbf{E}_{\text {std. }} \\
\text { psi }\end{array}$ & $\begin{array}{c}\text { Slope }_{\text {post- }} \\
\text { peak, psi }\end{array}$ \\
\hline \multirow{12}{*}{2} & 1 & 5728 & \multirow{12}{*}{$5,853.5$} & \multirow{12}{*}{1,291} & 323,190 & \multirow{12}{*}{304,768} & \multirow{12}{*}{66,186} & 466,733 \\
\hline & 2 & 6888 & & & 350,169 & & & $3,685,092$ \\
\hline & 3 & 8170 & & & 404,220 & & & $4,537,144$ \\
\hline & 4 & 5842 & & & 297,846 & & & 875,042 \\
\hline & 5 & 5336 & & & 333,163 & & & 127,628 \\
\hline & 6 & 5108 & & & 297,659 & & & 95,819 \\
\hline & 7 & 7133 & & & 385,775 & & & $1,093,309$ \\
\hline & 8 & 4479 & & & 206,728 & & & 151,453 \\
\hline & 9 & 4843 & & & 323,204 & & & 195,612 \\
\hline & 10 & 3,783 & & & 182,771 & & & 88,297 \\
\hline & 11 & 5,611 & & & 307,604 & & & 412,861 \\
\hline & 12 & 7321 & & & 244,887 & & & $2,981,888$ \\
\hline \multirow{5}{*}{3} & 1 & 5,257 & \multirow{5}{*}{6,445} & \multirow{5}{*}{1,071} & 202,611 & \multirow{5}{*}{254,752} & \multirow{5}{*}{31,610} & 139,780 \\
\hline & 2 & 5,679 & & & 229,923 & & & 90,749 \\
\hline & 3 & 7,218 & & & 267,814 & & & 956,548 \\
\hline & 4 & 4,750 & & & 234,695 & & & 76,265 \\
\hline & 5 & 7,674 & & & 268,243 & & & $1,737,336$ \\
\hline
\end{tabular}




\begin{tabular}{|c|c|c|c|c|c|c|c|c|}
\hline & 6 & 7,172 & & & 250,434 & & & 695,831 \\
\hline & 7 & 6,045 & & & 243,824 & & & 268,357 \\
\hline & 8 & 5,735 & & & 247,703 & & & 99,907 \\
\hline & 9 & 7,227 & & & 286,786 & & & 646,613 \\
\hline & 10 & 7,689 & & & 315,486 & & & $1,459,073$ \\
\hline \multirow{6}{*}{4} & 1 & 8,591 & \multirow{6}{*}{8,783} & \multirow{6}{*}{669} & 251,536 & \multirow{6}{*}{224,594} & \multirow{6}{*}{23,066} & 196,851 \\
\hline & 2 & 8,985 & & & 223,637 & & & 816,663 \\
\hline & 3 & 9,941 & & & 228,336 & & & $1,575,136$ \\
\hline & 4 & 8,016 & & & 195,593 & & & 18,670 \\
\hline & 5 & 8,857 & & & 247,448 & & & 301,116 \\
\hline & 6 & 8,309 & & & 201,013 & & & 174,027 \\
\hline
\end{tabular}


The complete uniaxial stress strain curve in compression for coal specimen is similar to that shown in Figure 3-1 (Salamon, 1969). This curve can be divided into two parts: The first part, OA, represents the un-failed state, where an increase in stress is associated with an increase in the resistance of coal. The second part, $\mathrm{AB}$, represents the failing state, where a further increase in the strain is accompanied by a decrease in the load carrying capacity that results from progressive degradation of the material strength and stiffness once the damage initiation has reached point A. Hence, the slope of the post-peak indicates the rate of degradation of material strength and stiffness.

Wawersik and Fairhurst (1970) found that the post-peak behaviors of the rocks studied may be divided into two classes. For class I behavior, fracture propagation is stable in the sense that work must be done on the specimen for each incremental decrease in loadcarrying capacity. For class II behavior, the fracture process is unstable or self-sustaining, to control fracture, energy must be extracted from the material. The post-peak slope for class II is higher than that of class I. Hence, a higher post-peak slope means fast degradation of the coal specimen and fast crack propagation and hence brittle failure, while a lower post-peak slope means slow degradation, a delay in crack propagation and hence ductile failure. 


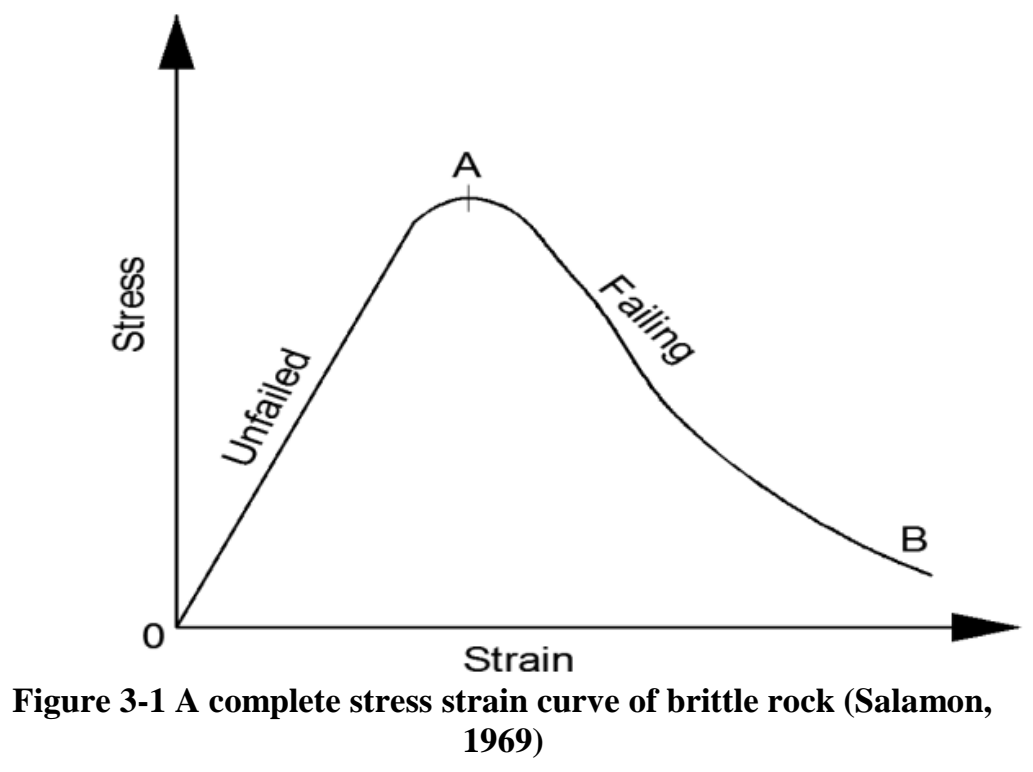

Therefore, the slope of the post-peak section of the stress-strain curve is a good indicator of whether the coal specimen is most likely to fail violently or non-violently. For a nonviolent failure, the coal specimen loses its strength gradually and gently and the slope of the post-peak is small and the failure of the coal specimen is controllable. Conversely, for violent failures, coal specimens lose their strengths suddenly, and the slope of the postpeak is large and the failure of the coal specimen is uncontrollable.

According to the laboratory test results shown in Table 3-1, it is obvious that the vertical strength, the modulus of elasticity and the slope of the post-peak of coal specimens vary considerably within coal specimens of the same width-to-height ratio. The variation in both the strength and modulus of elasticity decreases with increasing width-to-height ratios of the specimen. The standard deviation decreases with increasing width-to-height ratio, this means the degree of uncertainty about the strength and the modulus of elasticity decreases with increasing the width-to-height ratio.

As shown in Table 3-1, specimens \#3, \#7 and \#12 of width-to-height ratio = 2 have relatively high strength compared to the other coal specimens of the same width-to-height 
ratio. Their strength is almost equivalent to specimens of width-to-height ratio $=4$. Generally, the average strength of the specimen increases with increasing width-to-height ratios. It is obvious that a large value of the slope of the post-peak curve is not restricted to a certain width-to-height ratio.

The test results shown in Table 3-1 were exported to the Microsoft Excel 2010 to determine the correlation coefficient " $R$ " between the slope of the post-peak portion of the stress-strain curve and both the vertical strength and the Young's modulus of the coal specimens. "R" is used to measure the strength and the direction of linear relationship between two quantitative variables (Moor, 2009). It ranges from -1 to +1 . When "R" $=-1$, it means there is a perfect negative relationship between the two variables, i.e., as the value for one variable increases, there is a perfectly predictable decrease in value on the other variable. In other words, as one variable goes up, the other goes down. When " $R$ " $=+1$, the reverse relationship is true. Table 3-2 shows the correlation coefficients results for test results shown in Table 3-1. As mentioned before, the slope of the post-peak portion of the stress-strain diagram is a good indicator for potential violent failure.

Table 3-2 Correlation between post-peak slope of stress-strain curve and vertical strength and $\mathbf{E}$

\begin{tabular}{|c|c|c|}
\hline W/H ratio & R-with vertical strength & R-with E \\
\hline $\mathbf{2}$ & 0.835 & 0.608 \\
\hline $\mathbf{3}$ & 0.888 & 0.744 \\
\hline $\mathbf{4}$ & 0.955 & -0.015 \\
\hline
\end{tabular}

There is a strong positive correlation between the vertical strength of coal specimens and slope of the post-peak portion of the stress-strain curve as shown in Table 32. Hence, among coal specimens of the same $\mathrm{W} / \mathrm{H}$ ratio, the higher the strength, the higher 
the potential for violent failure. There is also a positive correlation between the modulus of elasticity and the slope of the post-peak portion of the stress-strain curve at width-to-height ratio equals to 2 and 3 . At width-to-height ratio $=4$, there is a weak correlation between the modulus of elasticity and the post-peak slope of the stress-strain curve. This indicates that the modulus of elasticity has no effect on whether the failure will be violent or not. Many researchers such as Ozbay (1989) thought that the width-to-height ratio only determines whether the failure is violent or not. According to Ozbay, pillars with width-to-height ratios of 4 to 5 can promote bumps, while a pillar with width-to-height ratio greater than 5 could not bump.

Therefore, coal specimens are more likely to fail violently if their vertical strength is very high, and their failure is often nonviolent when their vertical strength is low. Since most pillars have a width-to-height ratio more than 3 , according to the laboratory test results shown in Table 3-2, the unconfined compressive strength of the pillar has an influence on the potential for violent failure.

Figure 3-2 shows a how the vertical strength affects the post peak failure of coal samples of $\mathrm{W} / \mathrm{H}$ ratio $=3$. It is clear that the coal sample loses its strength suddenly and fail violently at the peak strength when its ultimate strength was attained as shown in Figure 32.a. The post-peak slope for those coal specimens that fail violently is close to 90 degrees. Conversely, the failure of coal specimen shown in Figure 3-2.b is non-violent. The vertical strength is small compared to the coal specimen that failed violently shown in Figure 3-2.a. The post-peak slope for this coal specimen is less than 90 degrees. The Young's modulus for the coal specimen which failed violently is relative close to that which failed non- 
violently. Possibly, this gives an indication that Young's modulus of the coal is not one of the main factors contributing to coal bumps.

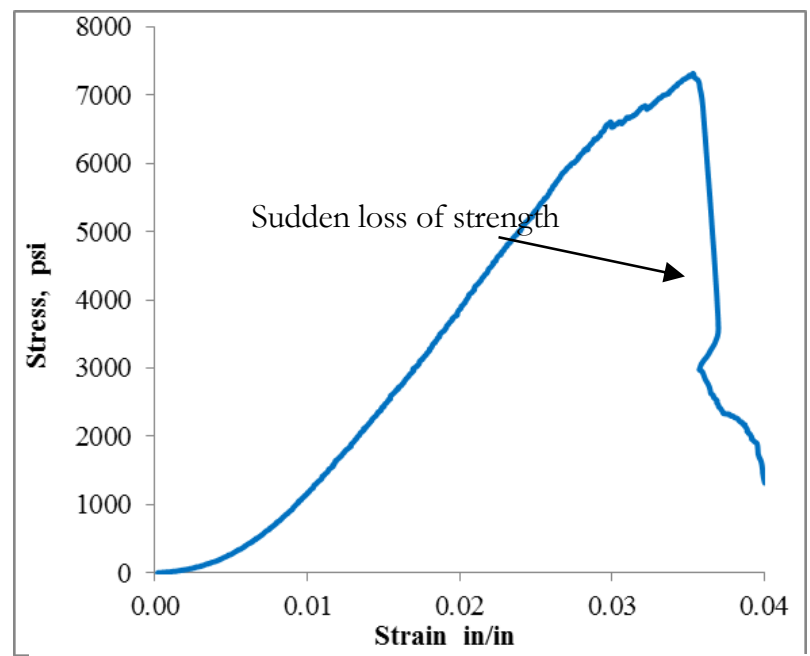

a) $\mathrm{W} / \mathrm{H}=3$ vertical strength $=7,321 \mathrm{psi} \quad \mathrm{E}=244,887$

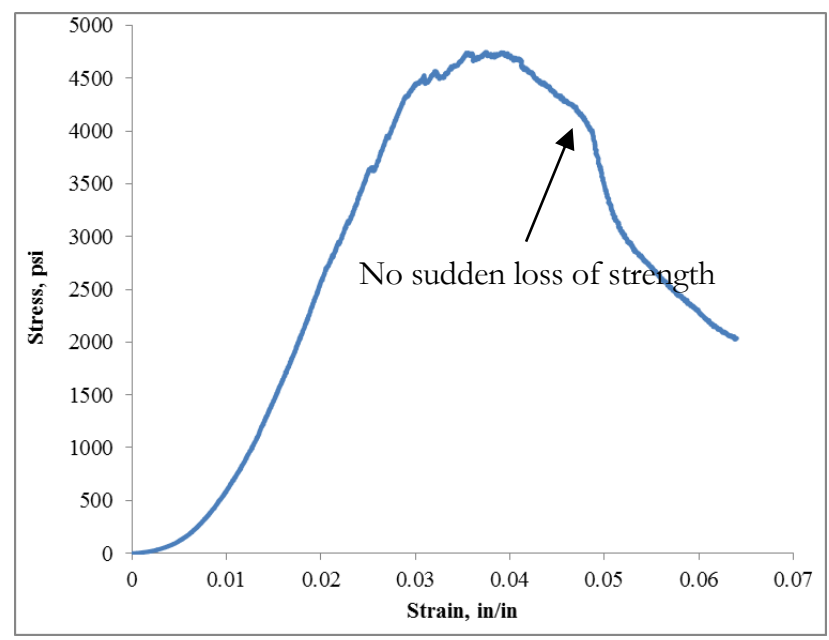

b) $\mathrm{W} / \mathrm{H}=3$ vertical strength $=4,750 \mathrm{psi} \quad \mathrm{E}=234,695 \mathrm{psi}$

Figure 3-2 Stress-strain curves for a) a violent failure and b) a nonviolent failure

\subsection{Computer Modeling for the Effect of UCS on the Potential for Violent} Failure

Computer modeling has been conducted to examine the effect of UCS on the potential for violent failure. 3D elastic-perfect plastic models were conducted using a finite 
element software package ABAQUS-6.10. The variation of energy released was examined for coal specimens of different UCS (4,000 psi, 2,000 psi, and 1,000 psi). The height and the width of the modeled coal specimens are 1.0 in and 4.0 in respectively, making the width-to-height ratio $=4$. An interface friction of 0.25 was assumed between the modeled coal specimens and upper and lower loading platens. Both the upper and the lower platens are assumed to be rigid bodies. The upper platen was fully restricted in all directions, while the lower platen was allowed to move 0.05 in in the vertical direction. The element size was kept constant in the three models to overcome the variation of the stress distribution due to the variation of the element size. Material response and failure follow the MohrCoulomb criterion. Table 3-3 shows the mechanical properties for the modeled coal specimens having different UCS's.

Table 3-3 Mechanical properties for the modeled coal specimens

\begin{tabular}{|c|c|c|c|c|}
\hline E, psi & $\boldsymbol{v}$ & $\begin{array}{c}\text { Friction } \\
\text { angle, degree }\end{array}$ & $\begin{array}{c}\text { Dilation angle, } \\
\text { degree }\end{array}$ & UCS, psi \\
\hline 250,000 & 0.25 & 30 & 17 & $4,000 \& 2,000 \& 1,000$ \\
\hline
\end{tabular}

Coal bumps are a manifestation and a result of the sudden release of the elastic energy in the coal under high stress (Peng, 2008). The significant role of the released energy was emphasized by Cook (1965) and Salamon (1984). They hypothesized that the excess potential energy causes damage known as rock bursts. According to Brady and Brown (1999) the released energy constitutes a basis for excavation design, and local rock fracture which frequently occurs around excavations consumes some or all of the released energy depending on the degree of fracturing. In the case of extensive rock fracture, all the released energy can be consumed in the rock disintegration. Moreover, the released energy can be considered as an index of the potential for local degradation, either in a stable way 
by yielding or an unstable way by bursting. So according to these researchers and others, the energy released is a good indicator for the violent failure of coal or rock.

Figure 3-3 shows how the elastic energy stored in the modeled coal specimens varies with changing the UCS of these specimens. It is quite obvious that the stored energy increases with increasing the UCS of the modeled coal specimen. The strain energy that can be stored in rock has been considered as some measure of the tendency to burst. The higher the maximum strain energy that can be stored in a given rock the more likely the rock will be subjected to bursting (Obert and Duval, 1967). Hence, the potential for violent failure increases with increasing UCS of coal

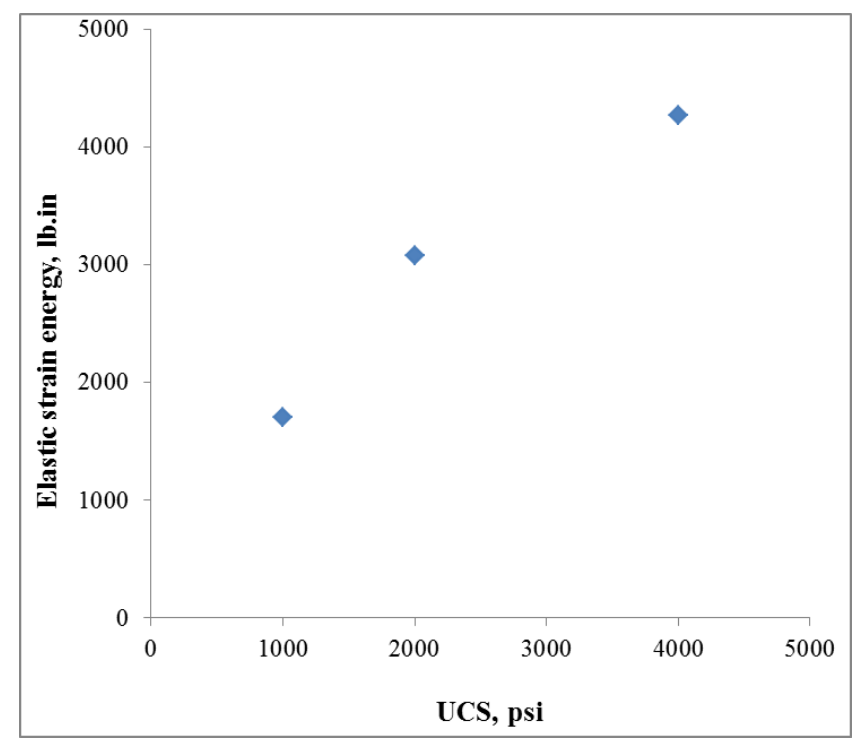

Figure 3-3 Variation of the elastic strain energy for modeled coal specimens with different $\mathrm{UCS}$ values

Babcock and Bickel (1984) suggested that many, if not most coals, can fail violently given proper conditions of stress and constraint. Brauner (1994) noticed that bursts occurred in almost all types of hard coal. Coal bumps can only occur in seams where the coal is able to store up high quantities of rock pressure in the form of elastic deformation (Baltz et al., 2008). It is well known that constraint increases the strength of 
coal. During a USBM study at the Castle Gate No. 3 mine (Barron, 1994), numerous bumps occurred on the longwall face and within the tailgate pillars where strong seam conditions coupled with very strong roof and floor conditions were observed.

Therefore, as demonstrated previously, the strength of coal specimens has an effect on the potential for violent failure. Next it is necessary to investigate whether this role is substantial or not. To accomplish that task a comparison between two kinds of coal from bump and non-bump prone mines was conducted.

It was decided to use cylindrical specimens of height to diameter ratio $=2$ to compare the mechanical properties for the Sunnyside seam, Utah coal and the Sewickley seam, WV coal.

\subsection{UCS for Cylindrical Coal Specimens for the Sunnyside seam, Utah}

The UCS of the Sunnyside coal seam, Utah coal has been determined from 20 cylindrical samples tested under a load control of $315 \mathrm{lb} / \mathrm{sec}$. These cylindrical coal samples have been sorted into two groups; the first group contains coal samples which are "free from macroscopic cracks", while the second group contains coal samples with clear macroscopic cracks. Although coal specimens in the second group have macroscopic crack, they are still intact. Figure 3-4a and b show examples of coal samples without and with macroscopic cracks respectively before testing by the WVU MTS machine. 

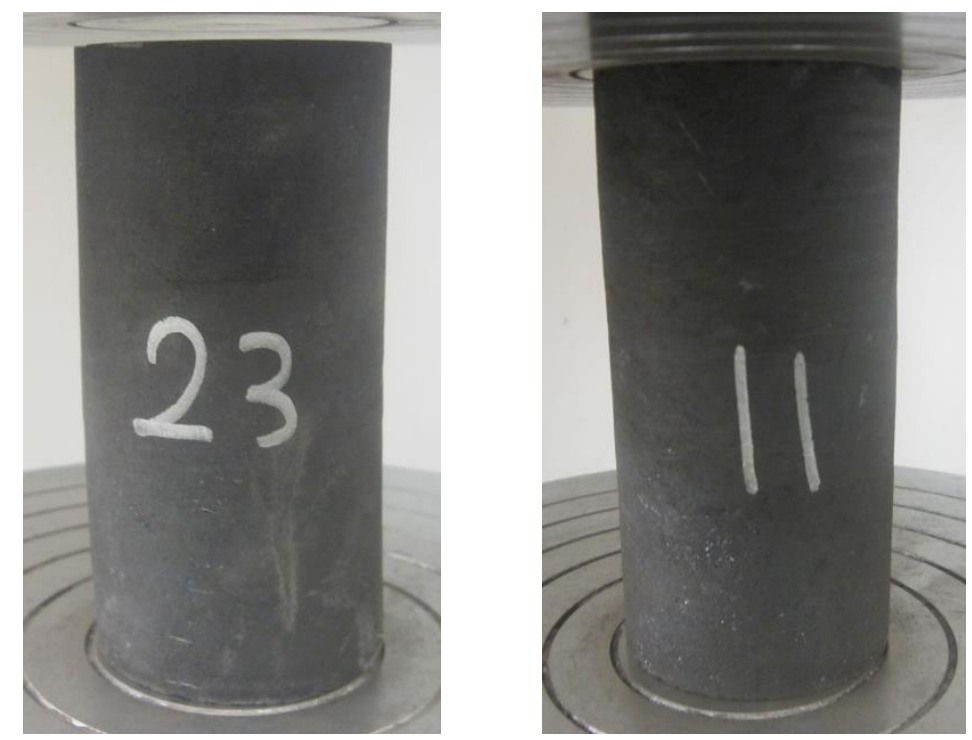

a) Coal specimens free from macroscopic cracks
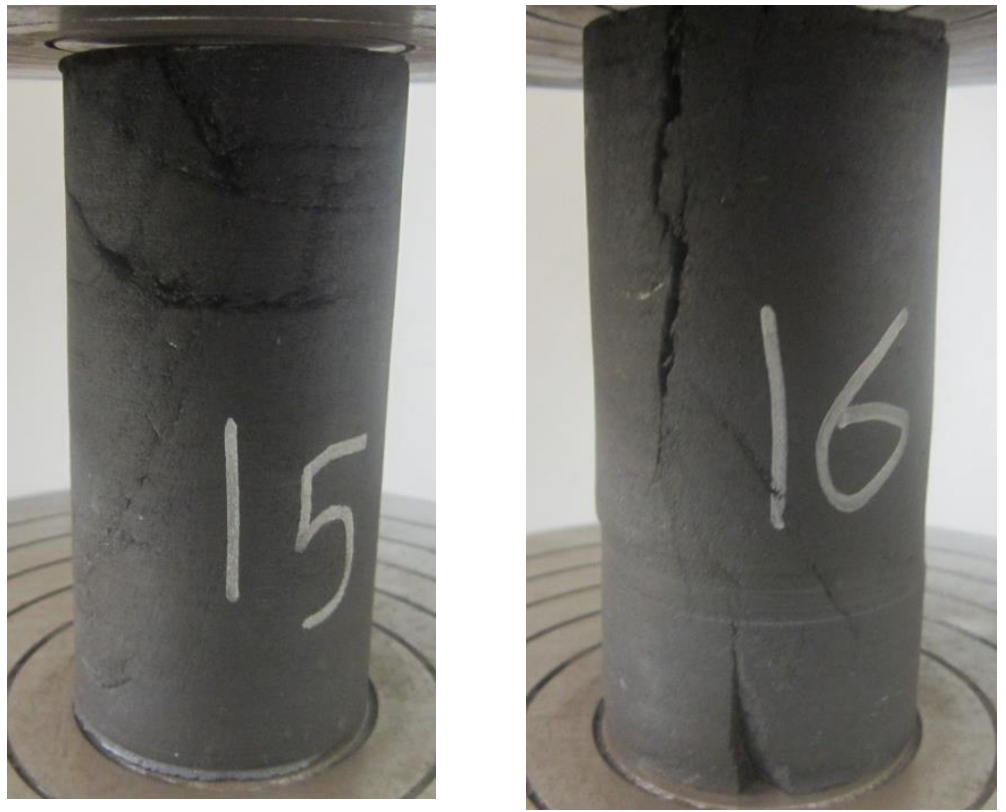

b) Coal specimens with clear macroscopic cracks

Figure 3-4 Coal specimens from the Sunnyside seam, Utah a) without macroscopic cracks and b) with macroscopic cracks

Table 3-4 shows the laboratory test results for the 20 cylindrical coal samples. The unconfined compressive strength, the modulus of elasticity and the density were 
determined from testing coal specimens of cylindrical shape of height-to-diameter ratio $=2.0$. The modulus of elasticity was calculated typically at $50 \%$ of the ultimate strength. The standard deviation was determined for both the UCS and the Young's modulus.

As shown in Table 3.4, the UCS for coal specimens without macroscopic cracks ranges from 2,536 psi to 4,680 psi with an average strength of 3,381 psi, while it ranges from 1,780 psi to 2,634 psi with an average strength of 2,280 psi for coal specimens with macroscopic cracks. There is a clear variation in the UCS for coal specimens with and without macroscopic cracks. Even within the same group, there is still a large variation. The standard deviation for coal specimens having macroscopic cracks is smaller than that for coal samples without cracks.

The average UCS for cylindrical coal samples without cracks is approximately 1.5 times that of the coal samples with macroscopic cracks, and the tangent Young's modulus for coal sample without cracks is approximately 1.33 times that of the coal samples with cracks. One-third of the cylindrical coal samples without macroscopic cracks have UCS more than 4,000 psi, which is relatively high for coal sample of height $/$ diameter ratio $=$ 2.0. 
Table 3-4 Laboratory test results for cylindrical coal specimen from the Sunnyside seam, Utah

\begin{tabular}{|c|c|c|c|c|c|c|c|c|c|c|c|}
\hline Sample \# & $\begin{array}{l}\text { H, } \\
\text { in }\end{array}$ & $\begin{array}{l}\text { D, } \\
\text { in }\end{array}$ & $\begin{array}{l}\text { H/D } \\
\text { ratio }\end{array}$ & $\begin{array}{l}\rho, \\
\mathrm{lb} / \mathrm{ft}^{3}\end{array}$ & $\begin{array}{l}\text { UCS, } \\
\text { psi }\end{array}$ & $\begin{array}{l}\mathbf{U C S}_{\text {avg }} \\
\text { psi }\end{array}$ & $\begin{array}{l}\mathbf{U C S}_{\text {std. }} \\
\text { psi }\end{array}$ & $\begin{array}{l}\text { E, tangent } \\
\text { psi }\end{array}$ & $\begin{array}{l}\mathbf{E}_{\text {avg }} \\
\text { psi }\end{array}$ & $\begin{array}{l}\mathbf{E}_{\text {std. }} \\
\text { psi }\end{array}$ & remarks \\
\hline 1 & 3.957 & 1.995 & 1.98 & -- & 4,680 & \multirow[t]{12}{*}{3,381} & \multirow[t]{12}{*}{797} & 536,504 & \multirow[t]{12}{*}{459,018} & \multirow[t]{12}{*}{70,447} & \multirow{12}{*}{$\begin{array}{l}\text { Samples } \\
\text { Without } \\
\text { Macro- } \\
\text { cracks }\end{array}$} \\
\hline 2 & 3.88 & 1.995 & 1.94 & -- & 2,641 & & & 423,934 & & & \\
\hline 3 & 3.972 & 1.994 & 1.99 & -- & 2,829 & & & 471,577 & & & \\
\hline 4 & 3.978 & 1.998 & 1.99 & 79.15 & 2,926 & & & 480,165 & & & \\
\hline 5 & 4.058 & 1.986 & 2.04 & 78.05 & 3,594 & & & 505,001 & & & \\
\hline 6 & 4.09 & 1.994 & 2.05 & 78.78 & 2,921 & & & 402,984 & & & \\
\hline 7 & 3.992 & 1.997 & 2.00 & 78.18 & 2,536 & & & 378,125 & & & \\
\hline 8 & 4.074 & 1.992 & 2.05 & 78.40 & 4,444 & & & 507,081 & & & \\
\hline 9 & 4.096 & 1.993 & 2.06 & 78.40 & 3,076 & & & 443,147 & & & \\
\hline 10 & 3.952 & 1.997 & 1.98 & 79.00 & 2,537 & & & 307,248 & & & \\
\hline 11 & 3.988 & 1.998 & 2.00 & 78.22 & 4,137 & & & 538,945 & & & \\
\hline 12 & 4.014 & 1.986 & 2.02 & 78.43 & 4,252 & & & 513,501 & & & \\
\hline 13 & 3.97 & 1.986 & 2.00 & 77.70 & 2,237 & \multirow[t]{8}{*}{2,280} & \multirow[t]{8}{*}{299} & 267,827 & \multirow[t]{8}{*}{343,755} & \multirow[t]{8}{*}{58,292} & \multirow{8}{*}{$\begin{array}{l}\text { Samples } \\
\text { With } \\
\text { Macro- } \\
\text { cracks }\end{array}$} \\
\hline 14 & 4.04 & 1.993 & 2.03 & 78.22 & 2,590 & & & 300,538 & & & \\
\hline 15 & 4.05 & 1.993 & 2.03 & 77.69 & 2,516 & & & 401,758 & & & \\
\hline 16 & 4.09 & 1.995 & 2.05 & 79.10 & 2,079 & & & 338,460 & & & \\
\hline 17 & 4.01 & 1.995 & 2.01 & 77.60 & 2,053 & & & 333,135 & & & \\
\hline 18 & 4.05 & 1.983 & 2.04 & 77.94 & 1,780 & & & 293,593 & & & \\
\hline 19 & 4.06 & 1.994 & 2.04 & 77.87 & 2,634 & & & 439,298 & & & \\
\hline 20 & 4.07 & 1.994 & 2.04 & 78.53 & 2,348 & & & 375,429 & & & \\
\hline
\end{tabular}


The minimum UCS for coal samples without cracks is very close to the maximum UCS for coal samples with macroscopic cracks. All cylindrical coal samples tested under load control of $315 \mathrm{lb} / \mathrm{sec}$ failed suddenly and violently, where the outer part or the rib zone was ejected away from the inner core of the sample. Figure 3-5 shows examples for the failure of cylindrical coal specimens after testing.
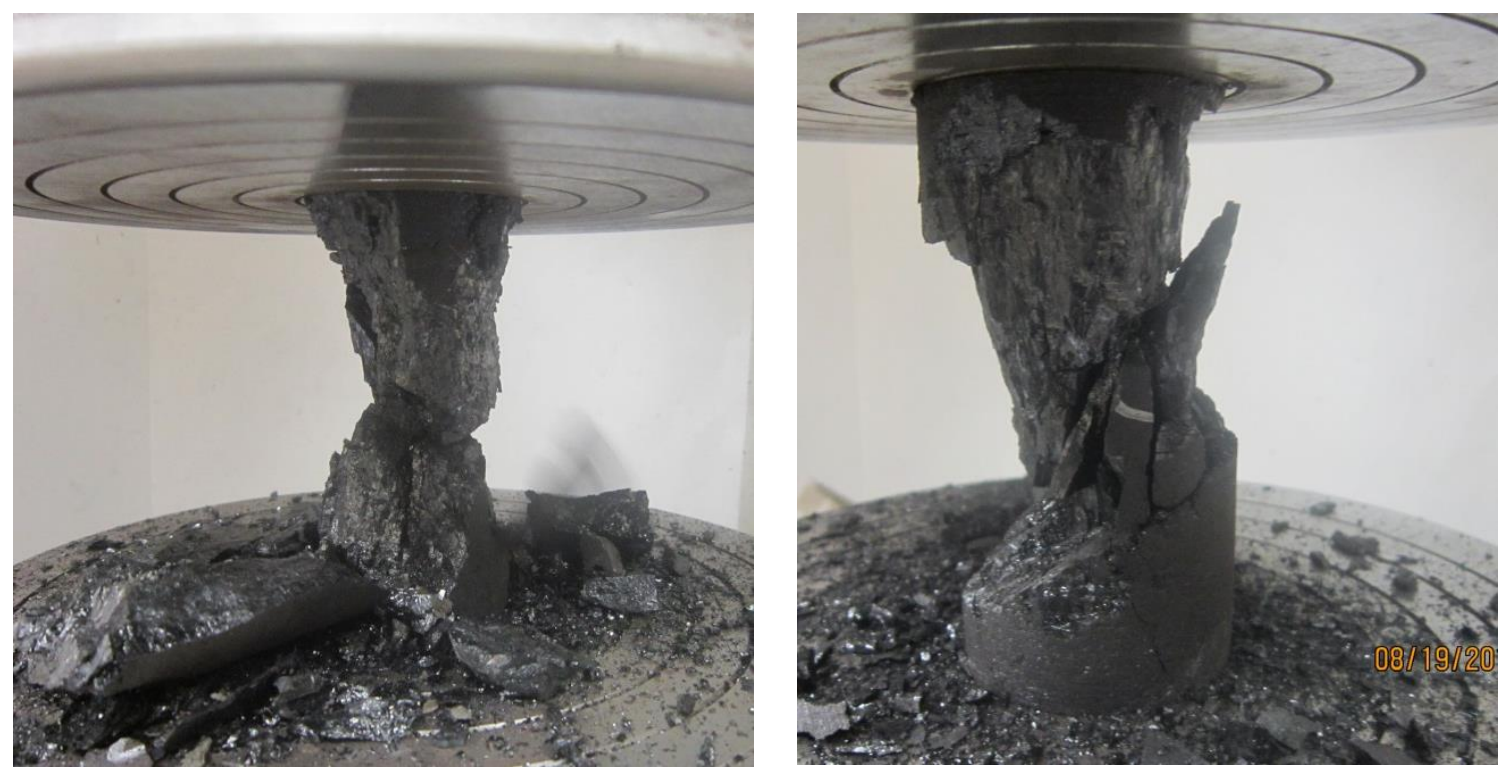

Figure 3-5 Cylindrical coal specimens from the Sunnyside seam, Utah after testing

Since, it was not possible to obtain cylindrical coal specimens from the WV coal mine; it is decided to use cubical specimens rather than cylindrical ones to examine the role of the mechanical properties on the potential for the violent failure.

\subsection{Specimens Preparation}

The laboratory experiments were conducted using coal samples obtained from a bump-prone, the Sunnyside seam, Utah. Coal samples were prepared using the normal techniques of cutting and grinding. Figure 3-6 shows the specimen preparation process. 
After the coal samples were obtained from the mine, they were cut into smaller cubes and cylinders. A grinding machine was used to make the surface smooth and flat. Cylindrical coal specimens were devoted for the shear tests, while the cubical samples were reserved for the UCS (the vertical strength) and the burst index tests.

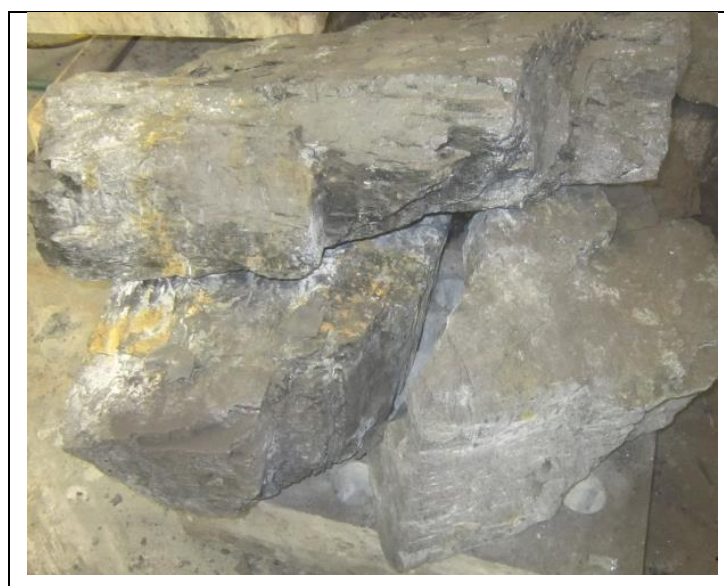

a. Coal samples to be cut and grinded

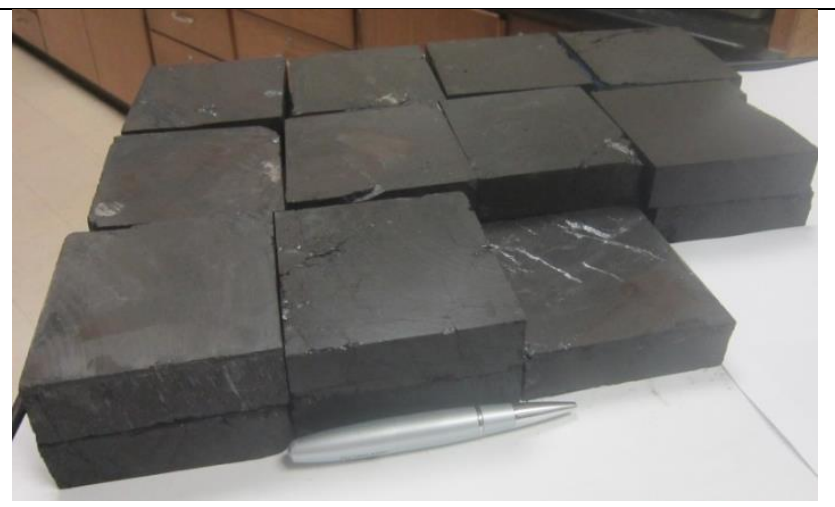

b. Coal samples after cutting and grinding

Figure 3-6 Two steps of coal sample preparation 


\subsection{Comparison of the Vertical Strength for the Sunnyside seam, Utah and Sewickley seam, WV}

To avoid variation of the strength with both shape and size, the vertical strength was determined from coal samples of the same shape (the width-to-height ratio =3) and the same size ( 3 in $x 3$ in $x$ lin).

Figure 3-7 shows the vertical strength for 52 coal samples from both the Sunnyside seam, Utah and the Sewickley seam, WV coals. The blue and red solid lines shown in Figure 3-7 represent the average vertical strength for the Sunnyside seam, Utah coal and the Sewickley seam, WV coal, respectively. The average vertical strength's for coal specimens from the Sunnyside seam, Utah and the Sewickley seam, WV are 7652 psi and 6360 psi, respectively. Hence the average vertical strength for the Sunnyside seam, Utah coal is approximately 1.2 times the average vertical strength for Sewickley seam, WV coal.

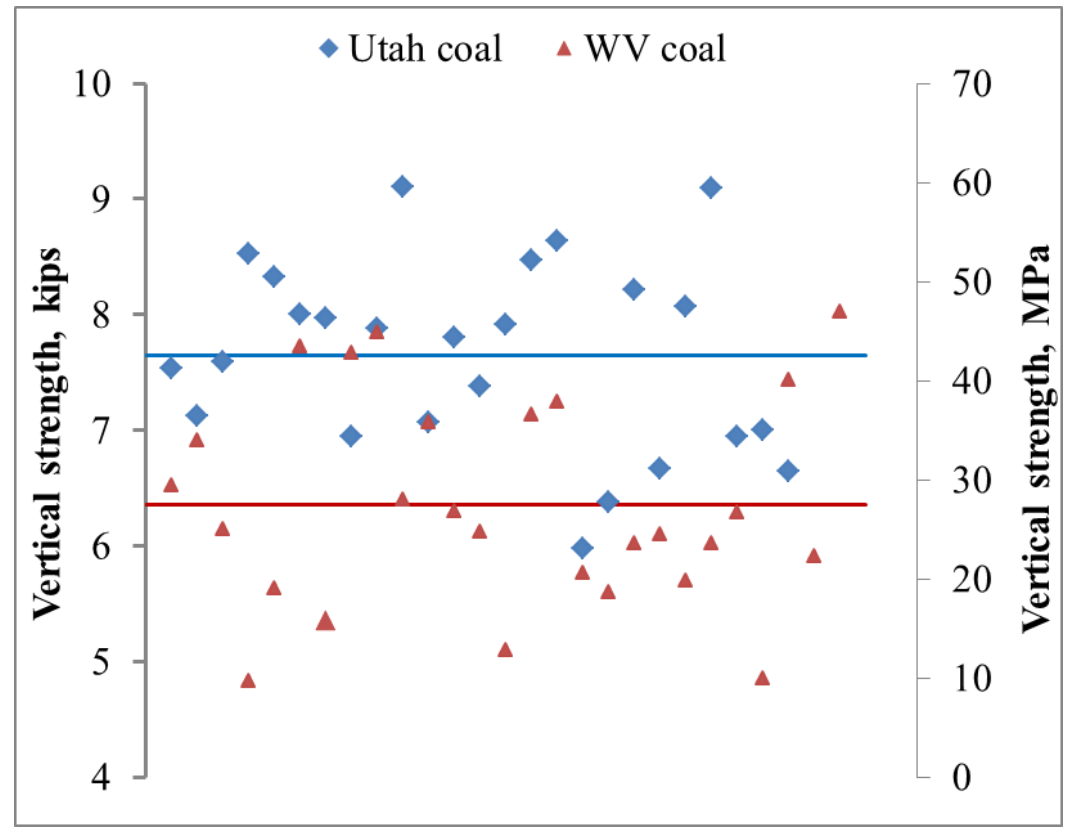

Figure 3-7 Comparing the vertical strength for the Sunnyside seam, Utah coal and the Sewickley seam, WV coal 
as shown in Figure 3-7, the average vertical strength for the Sunnyside seam, Utah coal is very close to the maximum vertical strength of the Sewickley seam, WV coal samples, and the average vertical strength for the Sewickley seam, WV coal is very close to the minimum vertical strength of the Sunnyside seam, Utah coal samples. The standard deviation for the Sewickley coal seam, WV coal is slightly higher than that for the Sunnyside seam, Utah coal, that's why the degree of scattering for Sewickley coal seam, WV coal is higher than the Sunnyside seam, Utah coal. In other words the degree of confidence is higher in the vertical strength of the Sunnyside seam, Utah coal samples.

According to Obert and Duvall (1967), the higher the strain energy that can be stored in a coal pillar, the more likely the coal will bump. The strain energy per unit volume in a uniaxially loaded coal sample is $\sigma^{2} / 2 \mathrm{E}$, where $\sigma$ is the uniaxial compressive strength of coal. Hence, with all mechanical properties identical except the UCS, the coal with the highest UCS would be more likely to burst. Consequently the potential for bumping is higher for the Sunnyside seam, Utah coal compared to the Sewickley seam, WV coal, because the average vertical strength for the Sunnyside seam, Utah coal is approximately 1.2 times the average vertical strength for the Sewickley seam, WV coal.

The question now is, would the $20 \%$ difference in the vertical strength between the Sunnyside seam, Utah coal and the Sewickley seam, WV coal cause a bump? In my point of view, the $20 \%$ difference in strength might not cause bump because the $20 \%$ of the average vertical strength for the WV coal is about $1272 \mathrm{psi}$, while the standard deviation, which is a measure for the dispersion, for the WV coal is 913 psi. Hence, the $20 \%$ difference in strength is relative close to the standard deviation of the coal, i.e., the $20 \%$ 
difference is not sufficiently high enough to make me unconfident to believe that that the UCS plays a substantial role in coal bumps.

Since the stress state in an underground mine is a tri-axial state, it may be necessary to consider the tri-axial strength of the coal specimens, rather than the UCS or the vertical strength. So the shear strength should be taken into consideration in addition to the UCS.

It is worth mentioning that the failure of the Sunnyside seam, Utah coal specimens is accompanied by high pitch noise or acoustic emission. In contrast, the failure of Sewickley coal seam, WV coal is accompanied by low noise. Unfortunately, no acoustic emission equipment was used during testing of the coal samples.

Tables 3-5 and 3-6 show the test results for the Sunnyside seam, Utah coal and the Sewickley seam, WV coal respectively.

Table 3-5 Test results for the Sunnyside seam, Utah coal

\begin{tabular}{|c|c|c|c|c|c|c|}
\hline Sample & $\begin{array}{c}\text { Vertical } \\
\text { strength, } \\
\text { psi } \\
\end{array}$ & $\begin{array}{c}\text { Vertical } \\
\text { strength- } \\
\text { Std., psi } \\
\end{array}$ & $\begin{array}{c}\text { Vertical } \\
\text { strength }_{\text {avge, }} \\
\text { psi } \\
\end{array}$ & $\begin{array}{c}\text { Young's } \\
\text { modulus, E, psi }\end{array}$ & $\begin{array}{l}\text { E-Std., } \\
\text { psi }\end{array}$ & $\mathbf{E}_{\text {avg, }}$ psi \\
\hline 1 & 7540 & \multirow{12}{*}{828} & \multirow{12}{*}{7,652} & 234863 & \multirow{12}{*}{25,612} & \multirow{12}{*}{305,661} \\
\hline 2 & 7127 & & & 291862 & & \\
\hline 3 & 7589 & & & 299456 & & \\
\hline 4 & 8530 & & & 329997 & & \\
\hline 5 & 8324 & & & 327966 & & \\
\hline 6 & 8008 & & & 284819 & & \\
\hline 7 & 7975 & & & 275598 & & \\
\hline 8 & 6941 & & & 297074 & & \\
\hline 9 & 7884 & & & 321487 & & \\
\hline 10 & 9103 & & & 309730 & & \\
\hline 11 & 7069 & & & 309854 & & \\
\hline 12 & 7801 & & & 327842 & & \\
\hline
\end{tabular}




\begin{tabular}{|l|l|}
\hline 13 & 7381 \\
\hline 14 & 7911 \\
\hline 15 & 8473 \\
\hline 16 & 8634 \\
\hline 17 & 5976 \\
\hline 18 & 6382 \\
\hline 19 & 8219 \\
\hline 20 & 6673 \\
\hline 21 & 8067 \\
\hline 22 & 9099 \\
\hline 23 & 6943 \\
\hline 24 & 7003 \\
\hline 25 & 6648 \\
\hline
\end{tabular}

\begin{tabular}{|l|}
\hline 294261 \\
\hline 318727 \\
\hline 328673 \\
\hline 322499 \\
\hline 288230 \\
\hline 272641 \\
\hline 320588 \\
\hline 285509 \\
\hline 330486 \\
\hline 348628 \\
\hline 325809 \\
\hline 274176 \\
\hline 320755 \\
\hline
\end{tabular}

Table 3-6 Test results for the Sewickley seam, WV coal

\begin{tabular}{|c|c|c|c|c|c|c|}
\hline Sample \# & $\begin{array}{c}\text { Vertical } \\
\text { strength, } \\
\text { psi } \\
\end{array}$ & $\begin{array}{c}\text { Vertical } \\
\text { strength- } \\
\text { Std., psi } \\
\end{array}$ & $\begin{array}{c}\text { Vertical } \\
\text { strength }_{\text {avg, }}, \\
\text { psi } \\
\end{array}$ & $\begin{array}{c}\text { Young's } \\
\text { modulus, } \\
\text { E, psi } \\
\end{array}$ & $\begin{array}{c}\text { E- Std., } \\
\text { psi }\end{array}$ & $\mathbf{E}_{\text {avg, }}$ psi \\
\hline 1 & 6520 & \multirow{12}{*}{913} & \multirow{12}{*}{6,360} & 316726 & \multirow{12}{*}{36,111} & \multirow{12}{*}{302,730} \\
\hline 2 & 6909 & & & 315120 & & \\
\hline 3 & 6142 & & & 263846 & & \\
\hline 4 & 4828 & & & 298975 & & \\
\hline 5 & 5633 & & & 297157 & & \\
\hline 6 & 7721 & & & 317725 & & \\
\hline 7 & 5357 & & & 255269 & & \\
\hline 8 & 7666 & & & 310632 & & \\
\hline 9 & 7853 & & & 314281 & & \\
\hline 10 & 6402 & & & 321291 & & \\
\hline 11 & 7072 & & & 361416 & & \\
\hline 12 & 6305 & & & 334880 & & \\
\hline
\end{tabular}




\begin{tabular}{|c|c|c|}
\hline 13 & 6119 & 314359 \\
\hline 14 & 5094 & 287816 \\
\hline 15 & 7139 & 327034 \\
\hline 16 & 7243 & 331156 \\
\hline 17 & 5771 & 299736 \\
\hline 18 & 5597 & 180931 \\
\hline 19 & 6019 & 352995 \\
\hline 20 & 6097 & 274227 \\
\hline 21 & 5696 & 310482 \\
\hline 22 & 6026 & 295257 \\
\hline 23 & 6285 & 311604 \\
\hline 24 & 4853 & 252486 \\
\hline 25 & 7436 & 312775 \\
\hline 26 & 5913 & 278683 \\
\hline 27 & 8026 & 336863 \\
\hline
\end{tabular}

The t-test, a statistical examination of two population means (the Sunnyside seam, Utah coal and the Sewickley seam, WV coal), was conducted using JMP-11. The t-value can be calculated from equation 3.1. The vertical strength test results shown in Tables 3-5 and 3-6 were exported to JMP-11 to determine if the average strength of the Utah and the WV coals are significantly different from each other. The t-test was conducted at a 0.95 confidence interval. Hence, the significance level is 0.05 . Note that the null hypothesis is that, there is no difference between average strength of the Utah and the WV coals, and the alternative hypothesis is that there is a significant difference between the Utah and the WV coals. 


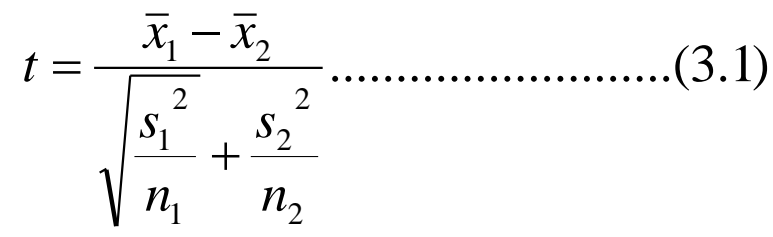

Where: $\mathrm{t}=\mathrm{t}$-value

$\bar{x}_{1}$ is the average strength for the Utah coal from the 25 test results shown in Table 3-5 and the WV coals.

$\bar{x}_{2}$ is the average strength for the WV coal from the 27 test results shown in Table 3-6.

$S_{1}$ and $S_{2}$ are the standard deviations for the Utah and the WV coals, respectively.

$\mathrm{n}_{1}$ and $\mathrm{n}_{2}$ are the number of samples from Utah and the WV coals respectively.

The P-value calculated from JMP was found to be less than 0.001. The p-value is defined as the probability of obtaining a result equal to or "more extreme" than what was actually observed. Since the P-value is smaller than the confidence level. Hence, the average strength of the Utah and the WV coals are significantly different from each other.

\subsection{Young's Moduli for the Sunnyside seam, Utah and the Sewickley seam, WV}

The tangent Young's modulus $\mathrm{E}_{\mathrm{t}}$ is the slope of the axial stress-axial strain curve at some fixed percentage, typically $50 \%$ of the peak strength (Brady and Brown, 1999). Figure 3-8 shows the tangent Young's modulus for the Sunnyside seam, Utah coal and the Sewickley seam, WV coal. The two solid lines in Figure 3-8 represent the average Young's modulus for the Sunnyside seam, Utah coal and the Sewickley seam WV coal. 


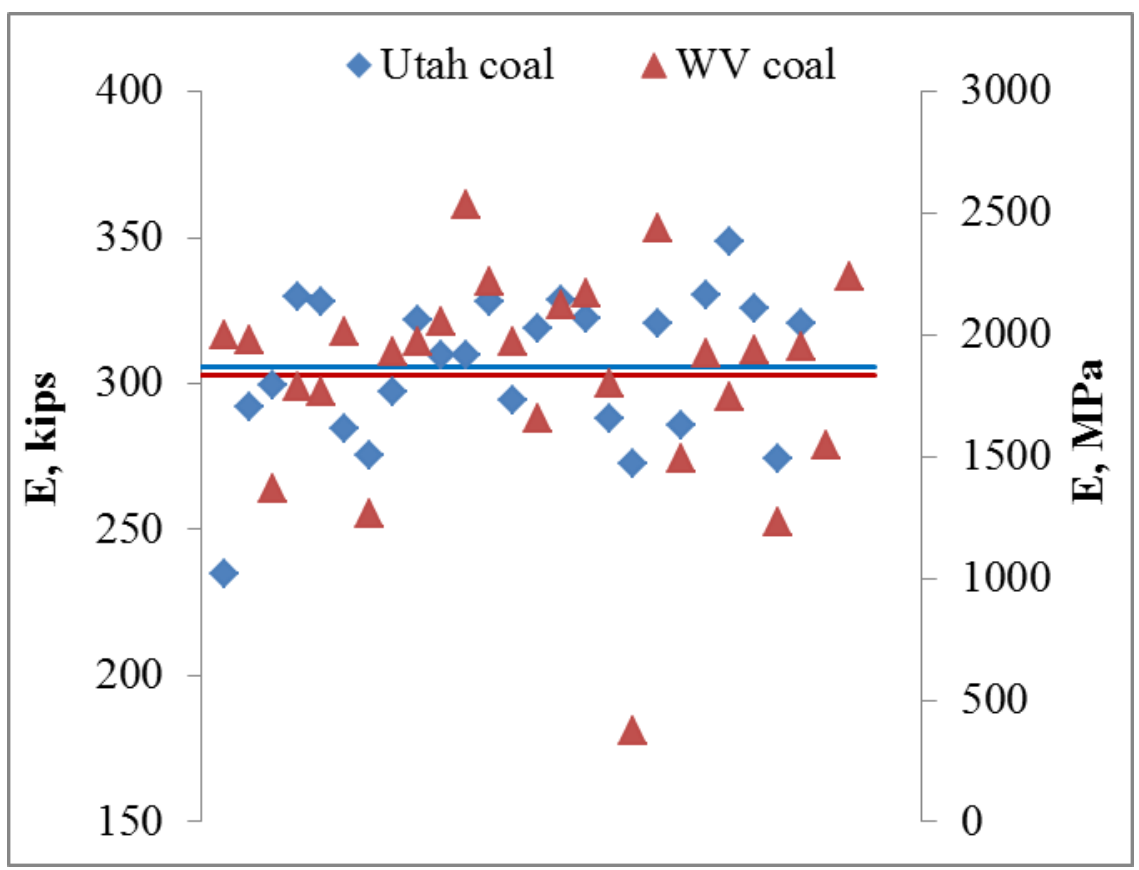

Figure 3-8 Comparing Young's moduli for the Sunnyside seam, Utah coal and the Sewickley seam, WV coal

The average Young's moduli for the Sunnyside seam, Utah coal and the Sewickley seam, WV coal are 305,000 psi and 302,000 psi, respectively as demonstrated by the blue and red lines shown in Figure 3-8. For the WV coal, there is one coal sample with a very low Young's modulus compared to the average value (red line). If this value is omitted from the test results, the average Young's modulus for the WV coal becomes 307,000 psi which is slightly higher than the Utah coal. Hence, the difference between the Young's modulus for Utah and WV coal is insignificant.

Maleki (1995) stated that most coal bumps have occurred in mines where the Young's modulus ratio ( roof- or floor-to -coal) was greater than 8 and the strength ratio (roof- or floor-to-coal) was greater than 4, severe bumps occurred at two room and pillar operations where Young's modulus and strength ratios were as low as 5 and 3, respectively. 
Since the difference in test results for Young's modulus between Sunnyside seam, Utah coal and Sewickley seam WV coal is insignificant, the Young's modulus of coal probably does not play a significant role in a bump.

\subsection{Shear Strength for the Sunnyside seam, Utah coal and the Sewickley seam WV coal}

To the author's knowledge, there is no data or results in the literature about the influence of shear strength parameters for coal on the potential for violent failure. The strength of coal or rock is not constant. Rather it depends on many factors and in particular the following three parameters: unconfined compressive strength, shear strength parameter (friction angle) and confining pressure. The variation of strength resulting from stress conditions can be represented by the Mohr failure envelope. According to Gadde (2003), the maximum principal stress at failure can be calculated from equations 3.2 and 3.3.

$$
\begin{aligned}
\sigma_{1} & =\sigma_{c}+k \sigma_{3} \ldots \ldots \ldots \ldots \ldots \ldots \ldots(3.2) \\
k & =\frac{1+\sin \varphi}{1-\sin \varphi} \ldots \ldots \ldots \ldots \ldots \ldots \ldots(3.3)
\end{aligned}
$$

Where: $\sigma_{1}=$ The maximum principal stress,

$\sigma_{\mathrm{c}}=$ Unconfined compressive strength

$\sigma_{3}=$ Minimum principal stress,

$\mathrm{K}=$ Tri-axial constant, and

$\phi=$ Internal friction angle of coal 
Figure 3-9 shows five stages necessary for preparing a direct shear test specimen using the GCTS Rock Direct Shear System RDS-200.

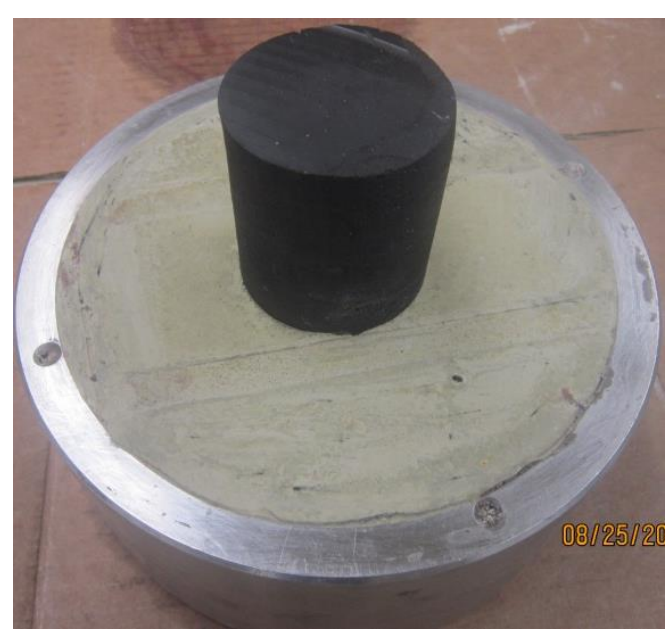

Stage-1

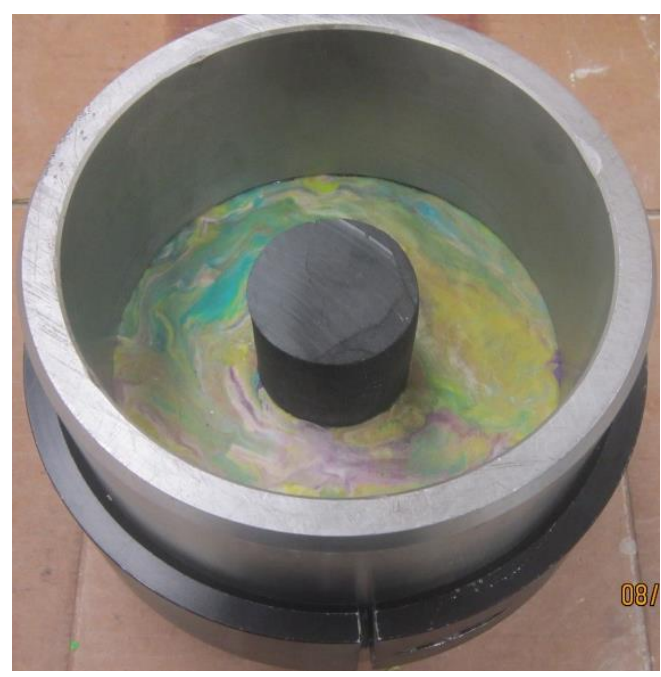

Stage-3

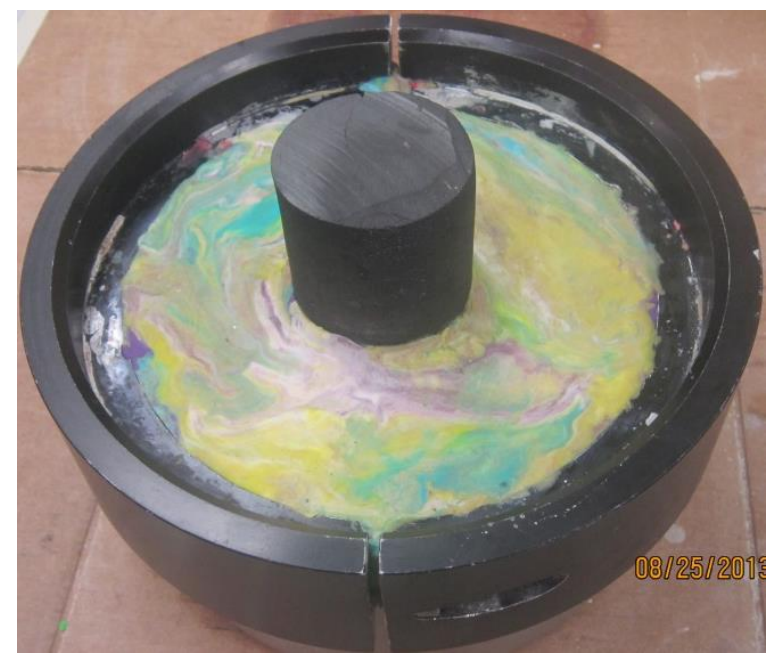

Stage-2

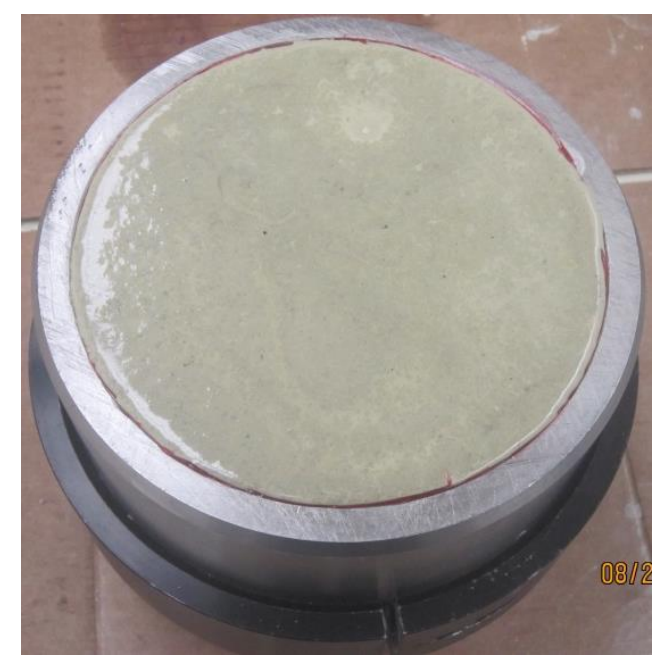

Stage-4 


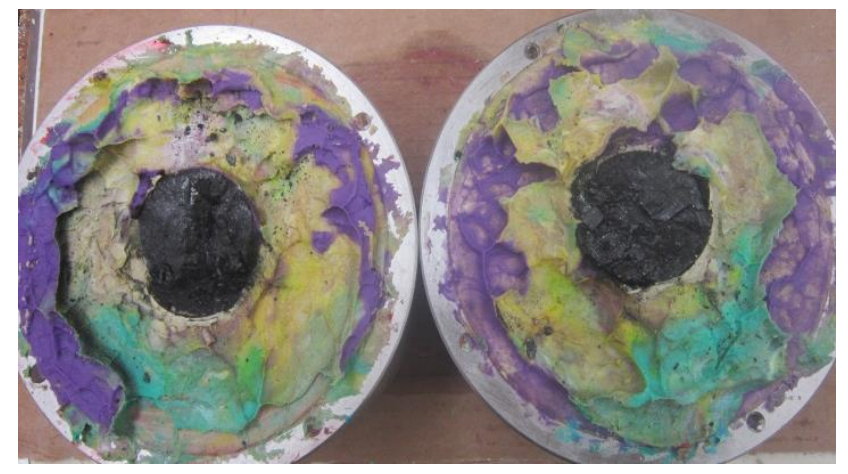

Stage- 5

Figure 3-9 Stages of sample preparation for direct shear tests

The GCTS Direct Shear Testing System RDS-200 (GCTS website) has been used to determine the frictional shear strength for 25 coal samples from the Sunnyside seam, Utah coal and the Sewickley seam, WV coal. As shown in Figure 3-10, the shear strength for Utah coal is higher than that for WV coal. The cohesion and friction angle for Sunnyside seam, Utah coal and Sewickley seam, WV coal are 436 psi and $39^{\circ}$, and 328 psi and $32^{\circ}$, respectively. These shear strength parameters affect the tri-axial strength of coal pillar. The higher shear strength of coal, the higher is the tri-axial strength. 


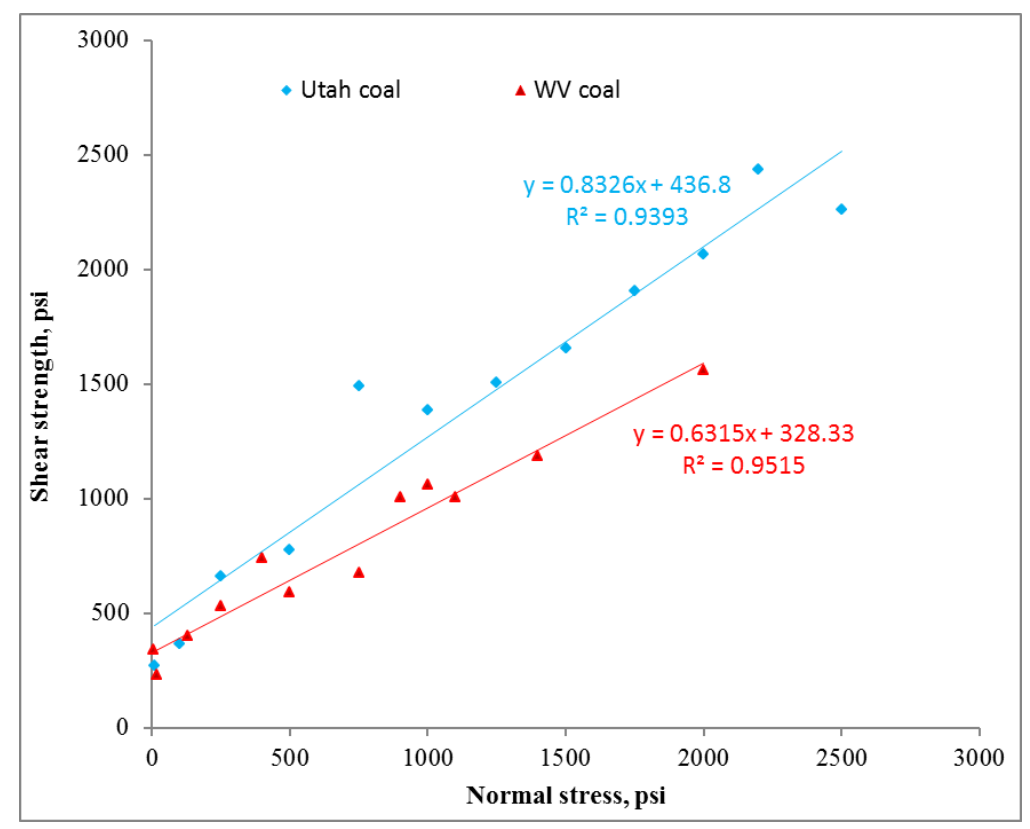

Figure 3-10 Comparing the shear strength for Utah and WV coals

As mentioned previously, the vertical strength of the Sunnyside seam, Utah coal is 1.2 times the vertical strength for Sewickley seam, WV coal. However, in underground mines, the state of stress is tri-axial not a uniaxial. So it is better to investigate the tri-axial strength using equations 3.2 and 3.3. Since the friction angles of the Sunnyside seam, Utah coal and the Sewickley seam, WV coal are $39^{\circ}$ and $32^{\circ}$, respectively. Consequently the triaxial constant, $\mathrm{K}$, would be approximately 4.4 and 3.3 for the Utah and the WV coals respectively by substituting in equation 3.3.

For the sake of explanation, assuming that $\sigma_{3}$ equals 1000 psi. By substituting in Equation 3.2, the tri-axial strength of the Sunnyside seam, Utah coal will be 1.25 times the tri-axial strength of the Sewickley seam, WV coal. It is obvious that, the tri-axial strength of the Sunnyside seam, Utah coal exceeds the tri-axial strength of the Sewickley seam, WV coal by $25 \%$, which in my point of view is not significantly high enough to be confident that the strength of coal plays a significant role in the bump. 
Campoli (1987) documented that a friable coal experienced bumps in both Mine-1 which is operating in the Pocahontas No. 4 Coalbed of the Pocahontas Formation and Mine-5 which is operating in the No. 2 Gas Coalbed of the Kanawha Formation Campoli's observation supports my point of view that the $25 \%$ difference in strength between the Utah and the WV is not enough to claim that the coal strength is one of the main causative factors for coal bumps.

\subsection{The Burst Index for the Sunnyside seam, Utah coal and the Sewickley seam WV coal}

The bump index is determined from the elastic hysteresis loop parameters in the uniaxial compression loading and unloading tests on rock specimens up to approximately $80 \%$ of the compressive strength. The ratio of the strain energy retained (the elastic energy, Ee) to the strain energy dissipated (the plastic energy, Ep) provides the burst index (Peng, 2008; Singh et al., 2008).

The total energy imparted to the coal specimen during loading is divided into two components: the first component is the elastic strain energy that is released immediately upon release of the applied load. The second component is the permanent strain energy that is irrecoverable upon release of the applied load. So, the tendency of coal to burst depends on its ability to accumulate elastic strain energy. And it decreases as the permanent strain energy increases. Table 3-7 shows the classification for bump susceptibility according to the burst index (Peng, 2008). 
Table 3-7 burst susceptibility depending on the burst index

\begin{tabular}{|c|c|}
\hline Degree of burst susceptibility & burst index \\
\hline Severely susceptible & $>5$ \\
\hline slightly susceptible & $2-5$ \\
\hline Not susceptible & $<2$ \\
\hline
\end{tabular}

Figure 3-11 shows an example of the loading-unloading stress-strain curve for the Sunnyside seam, Utah coal and the Sewickley seam, WV coal. The coal specimens were loaded up to $80 \%$ of their vertical strength strength which is approximately 55,000 lb for Sunnyside seam, Utah coal and 46,000 lb for Sewickley seam, WV coal (see Figure 3-7). The unloading stage starts as soon as the coal specimens reached the specified values.

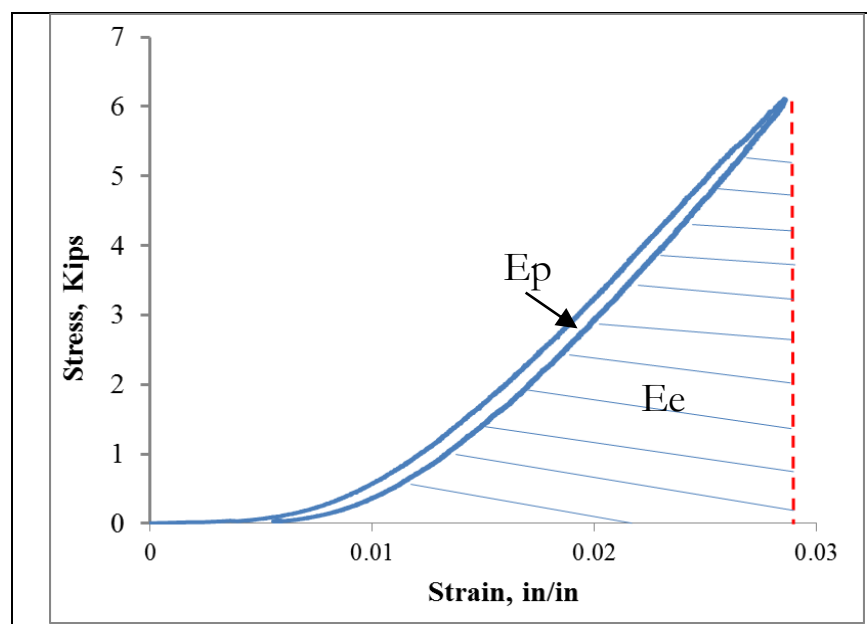

a- Utah coal

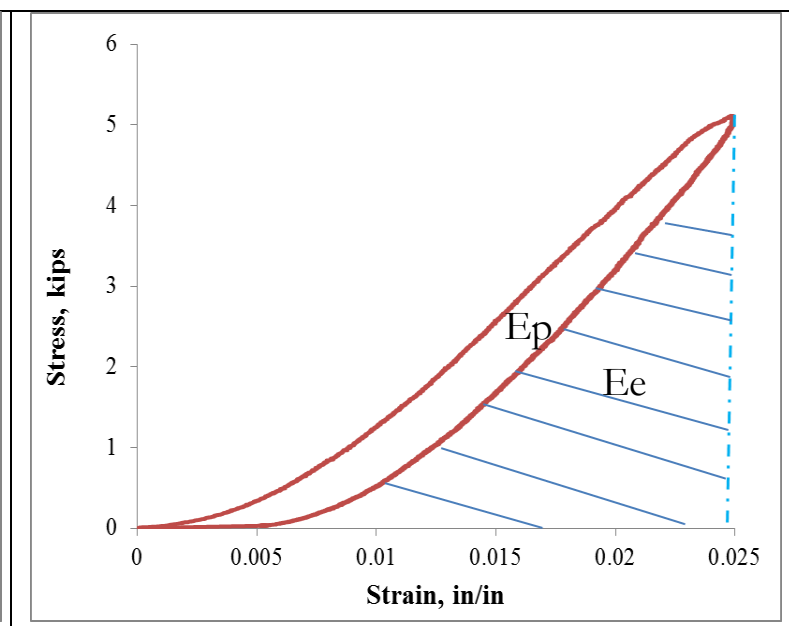

b- WV coal

Figure 3-11 Elastic and dissipated energy for a) the Sunnyside seam, Utah coal and b) the Sewickley seam, WV coal

As shown in Figure 3-11, for Sunnyside seam, Utah coal the plastic energy, Ep, is very small compared to the elastic energy, Ee, stored in the coal specimens. This is why its burst index is higher. For Sewickley seam, WV coal the plastic energy, Ep, is slightly larger. That is why its burst index is lower. 
Table 3-8 shows the burst index results for the Sunnyside seam, Utah coal and the Sewickley seam, WV coal. The burst index for all specimens from the Sunnyside seam, Utah coal is more than 5, which is the threshold limit between severely and slightly susceptible to bump shown in Table 3-7. All samples from the Sunnyside seam, Utah coal would be classified as severely susceptible to bump according to Table 3-7.

Table 3-8 burst susceptibility index for the Sunnyside seam, Utah and Sewickley seam WV

\begin{tabular}{|c|c|c|c|c|}
\hline Coal seam & $\begin{array}{c}\text { Sample } \\
\#\end{array}$ & burst index & Classification & Avg. burst index \\
\hline \multirow[b]{2}{*}{ Sunnyside seam, } & 1 & 9.85 & Severely susceptible & \multirow[t]{4}{*}{10.71} \\
\hline & 2 & 12.24 & Severely susceptible & \\
\hline \multirow[t]{2}{*}{ Utah coal } & 3 & 7.59 & Severely susceptible & \\
\hline & 4 & 13.16 & Severely susceptible & \\
\hline \multirow{4}{*}{ Sewickley seam, } & 1 & 3.69 & Slightly susceptible & \multirow[t]{4}{*}{3.25} \\
\hline & 2 & 2.79 & Slightly susceptible & \\
\hline & 3 & 2.52 & Slightly susceptible & \\
\hline & 4 & 4.03 & Slightly susceptible & \\
\hline
\end{tabular}

It might be reasonable to classify Sunnyside seam, Utah coal as very severely susceptible to bump, because its bump index is significantly higher than the threshold limit recommended for severely susceptible to bump shown in Table 3-7. All specimens from Sewickley seam, WV coal are classified as slightly susceptible to bump, because the bump index for these samples is between 2 and 5. The average burst index for Sunnyside seam, Utah coal is more than three times that for Sewickley seam, WV coal. 
Generally the Utah coal has the ability to store more elastic strain energy, Ee, and dissipate little energy, Ep, compared to WV coal. The mechanical characteristic that seems to distinguish between bump and non-bump prone coal is that the non-bump prone coal tends to plastically deform under stress such that when failure occurs, it takes place slowly.

The calculated burst index matched well with field observations. The Sunnyside seam, Utah coal is classified as severely susceptible to bump experienced bumps several times. However, the Sewickley seam, WV coal which is classified as slightly susceptible to bump does not experience any bumps. Therefore, the burst index is a reliable method to distinguish between bump and non-bump prone coal. According to Brauner (1994), the bump index is a more reliable indicator than the UCS to predict whether a coal is liable to bump because he found that coal of low to medium strength, 1,523 psi to 2,147 psi, exhibited severe bursts.

\subsection{Summary for Chapter Three}

The test results performed in this research showed that the bump index is a reliable method to distinguish between bump-prone and non-bump-prone coal (the Sunnyside seam, Utah coal and the Sewickley seam WV coal). According to the bump index, the Sunnyside seam, Utah coal is classified as severely susceptible to bump because it has the ability to store more energy, while dissipating little energy. The UCS of Sunnyside seam, Utah coal is 1.2 times greater than the vertical strength of Sewickley seam, WV coal. In my point of view, the $20 \%$ difference in the vertical strength is not sufficient to determine bump-prone or non-bump-prone. When the frictional shear strength is added to the vertical strength, the tri-axial strength for the Sunnyside seam, Utah coal becomes 1.25 times the tri-axial strength of the Sewickley seam, WV coal. This is still not significantly high 
enough to be confident that the strength of coal plays a significant role in bump. The Young's modulus is almost the same for both Sunnyside seam, Utah coal and Sewickley seam, WV coal. Hence, it is unlikely to play a significant role in the coal bumps.

Therefore, the coal itself plays an important role in coal bumps. However, that role "alone" is not sufficient for the occurrence of bumps, and hence the coal itself is not one of the main causative factor for coal bumps. That means the local variation of the geological conditions might be one of the main factors controlling coal bumps, hence future research in bump should focus more on these geological conditions surrounding the pillars. 


\section{CHAPTER 4: UNDERSTANDING COAL BUMP MECHANISMS}

\subsection{Introduction}

In order to mitigate and control coal mine bumps, it is necessary to thoroughly understand the coal bump mechanisms. Several theories have been proposed for coal mine bumps (Babcock and Bickel, 1984; Holland, 1958b; Iannacchione and Zelanko, 1994; Maleki, 1995; Peng, 2008; Rice, 1935). Two common mechanisms for coal bumps received the most attention among researchers and practitioners. The first one was proposed by Holland (1958b): When a coal pillar is loaded close to its ultimate strength under constrained conditions, a sudden loss of interface friction would cause coal bumps. The second mechanism for coal bumps was proposed by Rice (1936): It is known as a shock bump due to breaking of the overlying thick massive strata close to the coal seam, causing a great hammering effect similar to an impact load, resulting in a shock wave transmitted to the coal pillars underneath. Although these mechanisms were proposed many decades ago, little work has been done to examine the impact of the first proposed mechanism on the violent failure of coal pillar and almost no work has been done to examine the second mechanism. Hence, the main objective of chapter four is to better

understand the bump mechanisms. The energy changes that take place during sudden loss of constraint or sudden impact load are utilized to understand why bumps would occur. 


\subsection{Examining Why Sudden Loss of Friction Would Cause Bumps}

According to Cook (1963), Meikle (1965), and Prassetyo et al. (2011) the strength of a coal or rock pillar decreases with decreasing interface friction. Practically, the interface friction provides the inherent strength of the pillar with supplemental strength that increases with increasing the interface friction due to the confinement. Babcock and Bickel (1984) proposed that the sudden loss of friction might cause violent failures and most coals can be made to burst if appropriate conditions of stress and constraint are present. This finding was one step further than that of Holland (1958b), who had first proposed the concept. However this finding did not answer the important questions such as: How is a pillar affected by the sudden loss of friction? Where are the critical regions in the pillar that are more susceptible to violent failure? And how do strain energies change due to loss of constraint?

3D Elastic Finite Element models have been conducted using ABAQUS 6.10. The elastic modulus, Poisson's ratio, and density of the modeled coal specimen are 250,000 psi, 0.25 , and $84 \mathrm{lb} / \mathrm{ft}^{3}$, respectively. The purpose of these models is to examine the energy released and transient stress associated with a sudden loss of constraint between the modeled coal specimen and rigid platens. To consider how the sudden release of an elastic energy could cause a bump, it is assumed that a coal specimen is similar to a spring under compression. The more load is applied to compress the spring; the more elastic energy will be stored in that spring. If the load is released from the spring instantaneously, the spring will rebound and the stored elastic energy will release suddenly. Losing constraint for coal specimen is similar to releasing load from a spring under compression. So the elastic energy stored in the coal specimen will release instantaneously and violently. 
Coal samples of square cross-section, and a height of 1.0 in and a width of 4.0 in were modeled using ABAQUS 6.10. Two coefficients of friction (0.25 and 0.10) were used to examine how sudden change in interfaces friction would cause the violent failure. The complete load history of the computer simulation is divided into two stages. During the first stage, static analysis has been performed. The load was ramped up smoothly by forcing the lower platen to move 0.1 in in the vertical direction. The initial value for coal/platens interface friction was either 0.25 or 0.1 . The linear Coulomb friction model without limit for the interfacial shear stress was assumed. During the second stage of loading, the coal/platens interface friction in the whole surface was removed completely and suddenly. The short-term response of the coal specimen to the induced transient stress resulting from losing constraint requires dynamic analysis to be performed. That is why ABAQUS implicit dynamic was used in the second stage of loading. The time period for that stage was $0.001 \mathrm{sec}$. The final load reached in the first stage was kept constant and propagated through the second stage.

Figure 4-1 shows the variation of the elastic strain energy with time at two coefficients of friction and $\mathrm{W} / \mathrm{H}$ ratio $=4$. At time zero, zero $\%$ of the external load was applied, and at time 1, $100 \%$ of the load was applied. During the first stage of loading, the elastic strain energy increases with increasing applied load while the kinetic energy is zero. The elastic strain energy increases with increasing the coefficient of friction. During the second stage, there is a rapid energy change and a dynamic response associated with sudden loss of constraint. Examples for the transient effects are the sudden release of elastic energy and sudden increase of kinetic energy, non-zero value for the kinetic energy associated with losing constraint indicates that losing constraint is not a static process. 

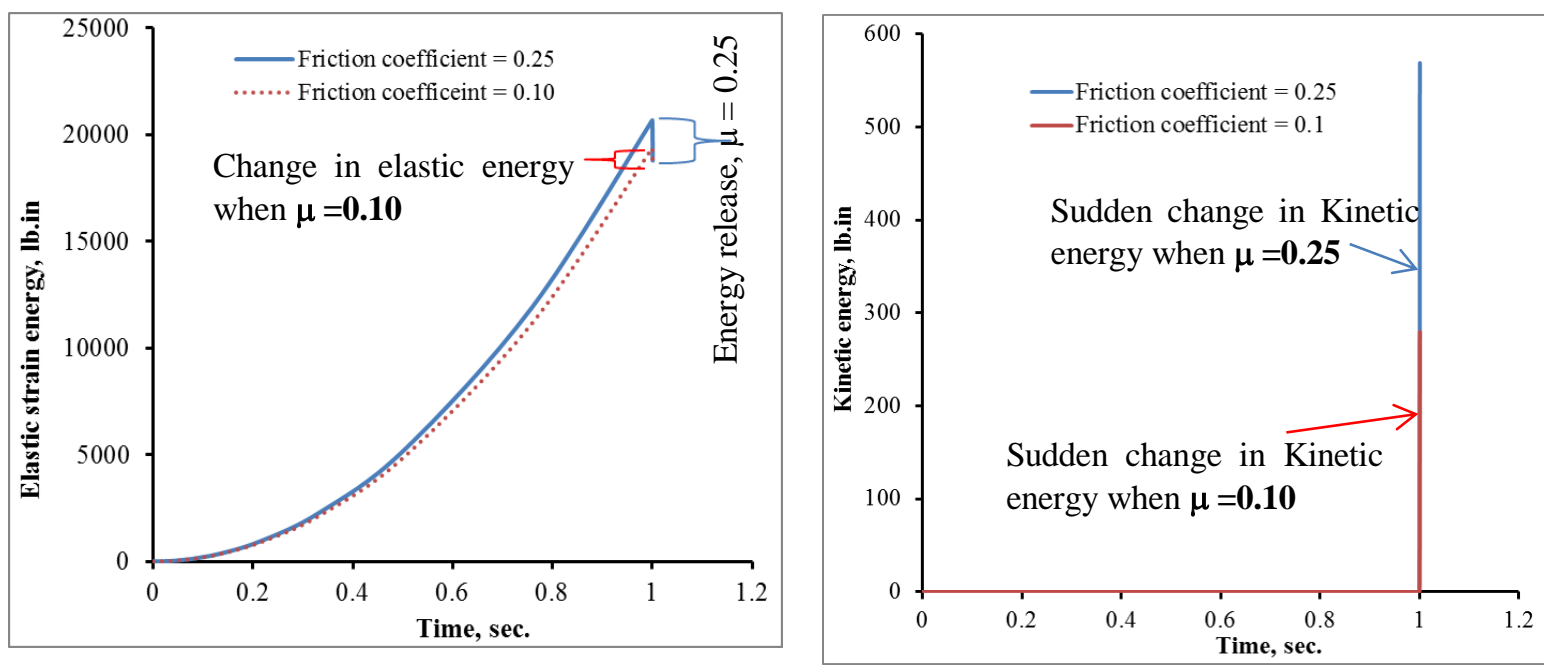

$\mathrm{W} / \mathrm{H}$ ratio $=4$

Figure 4-1 Variations of released and kinetic energies due to sudden loss of friction

The amount of energy released and kinetic energy increase with increasing the interface friction. Hence, the degree of violence increases with increasing interface friction. That is not a new finding, because Prassetyo et al. (2011) found that the potential for violent failure increases as the interface friction increases when he was investigating the effect of interface friction on the violent failure of coal specimens.

Griffith (1921) realized that a crack in a body would not grow unless energy was released to overcome the energy needed for forming two new surfaces. Hence both the released elastic and kinetic energies are necessary for the occurrence of coal bumps. Obviously, the released elastic energy is consumed in the initiation and/or propagation of existing cracks inside the body, while the kinetic energy provides the edges with sufficient energy for ejection from the coal specimen.

To get a complete picture about the adverse impact of sudden loss of interface friction, it is necessary to study how the vertical stress changes in a coal specimen before and after losing constraint. The vertical stress was studied at three specified paths of 
modeled coal specimen of $\mathrm{W} / \mathrm{H}$ ratio $=4$, and interface friction $=0.25$ as shown in Figure 4-2a. Path 1-1 is located on the middle of the top surface where the vertical load was applied. Path 2-2 is located at the edges corners where the front and side surfaces meet. Path 3-3 is located at the middle of the front surface of the modeled coal specimen.

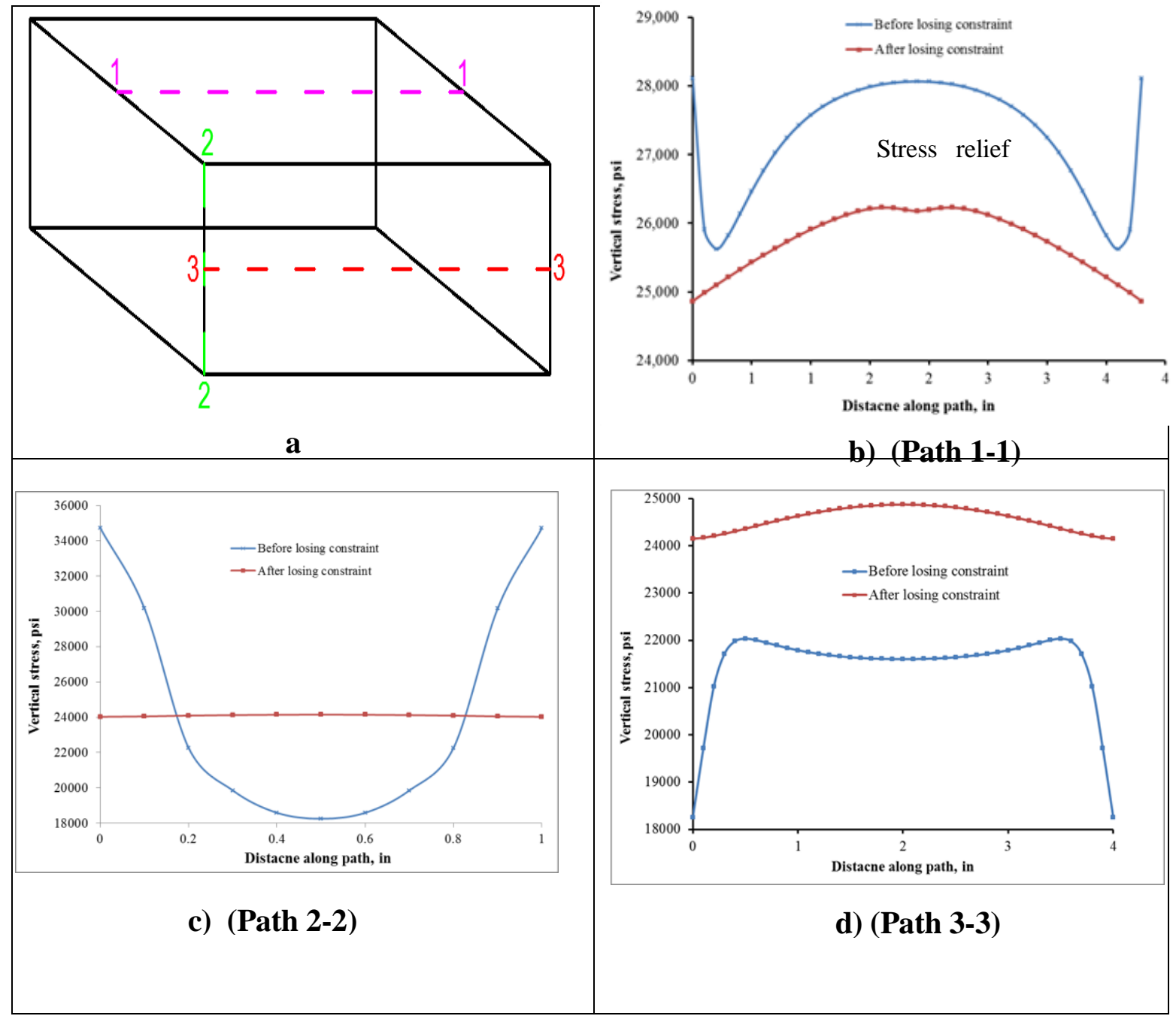

Figure 4-2 Variation of the vertical stress at different paths due to losing interface friction

As shown in Figure 4-2, there are rapid changes in the vertical stress due to sudden loss of interface friction. Some regions of the simulated coal specimen are subjected to transient increase in stress that would cause violent failure when it exceeds the final static strength, while other regions are subjected to sudden stress relief. Figure $4-2 b$ shows the 
variation of the vertical stress through path 1-1. Before losing the constraint, the maximum vertical stress occurs at the edges and the center of the coal specimen.

Since the strength of the central portion of coal specimen is higher than the strength of the boundary, failure will commence at the pillar boundary and migrate towards the center (Wagner, 1980). After losing constraint, there is a sudden rebound from maximum axial compression. The final vertical stress on the top surface is not the same at the edges and the center of the modeled coal specimen. The central portion is subjected to more vertical stress, which indicates that the constraint was not lost completely in the central portion, because the time period proposed for losing constraint in the whole surface is not long enough. Unfortunately, increasing the time period changes the analysis from dynamic to quasi-static or static, i.e., the dynamic response could not be captured if the time period is long. Figure 4-2c shows the variation of vertical stress before and after losing constraint through path 2-2. After losing constraint, the top and the bottom portions of the coal specimens near the platens are subjected to very high stress relief, $10 \mathrm{kpsi}$. While the middle portion of the vertical edge across path $2-2$ is subjected to very high stress concentration, $6 \mathrm{kpsi}$. These transient changes in the stress state generate shock waves, which might cause violent failure if the coal specimen is loaded near to its ultimate static strength. It is worth mentioning that, after losing constraint, the vertical stress is the same everywhere, which indicates that the edges can lose their constraint faster than the core. Figure $4-2 d$ shows the vertical stress at path 3-3. It is quite obvious that this portion of the coal specimen is subjected to a very high stress concentration especially at the edges due to losing constraint. These edges will definitely fail. 
Hence releasing the interface friction between the simulated coal specimen and the platens is similar to applying an impact load on the coal specimen. The moment the load is applied, the elastic energy will be a maximum and when the specimen has rebounded from its maximum displacement, moving back in the opposite direction, the coal specimen relaxes and strain energy stored in the coal specimen becomes available to perform work.

\subsection{Examining Why A Sudden Impact Load Would Cause Violent Failure}

Rice (1935) proposed this mechanism and called it a shock bump. So far, no work has been done to advance this theory. Bump prone coal seams are often overlain by strong and stiff stratum or strata such as sandstone or conglomerate (Campoli, 1987; Peng, 2008). In this section, the effect of impact load on the potential for violent failure has been studied.

A 3D elastic model has been conducted to study the effect of an impact load on the released elastic and kinetic energies for a coal specimen of $\mathrm{W} / \mathrm{H}$ ratio $=4$ and the interface friction between the coal specimen and the platen $=0.25$. The simulated coal specimen has square cross-sectional area of 4.0 in $\times 4.0$ in and a height of $1.0 \mathrm{in}$.

The Explicit dynamics analysis is required to analyze high-speed dynamic events such as impact loads. In dynamic analysis, the load is time dependent. The boundary conditions are similar to the former models used in Section 4.2, where the upper rigid platen is fixed and the lower platen can move only in the vertical direction. 


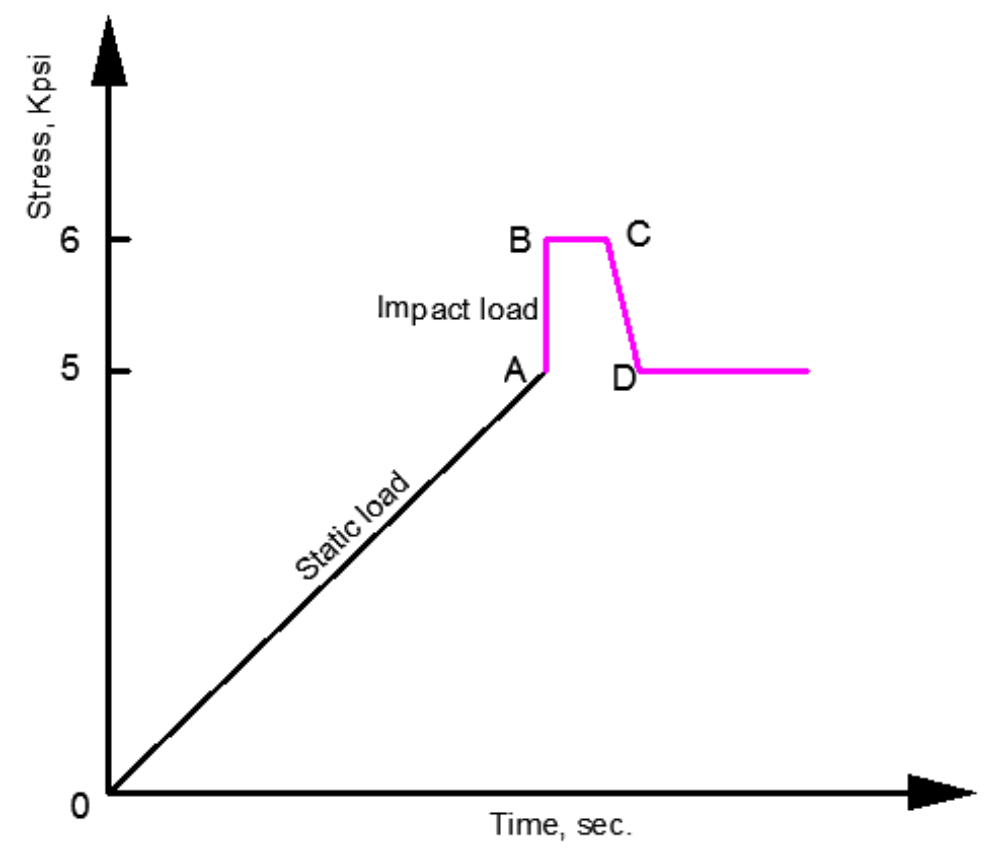

Figure 4-3 The applied load history of modeling from the start to the end of the analysis

Figure 4-3 shows the load history from the start to the end of the analysis. It can be divided into two stages. In the first stage where the static analysis was adopted, the load was ramped up smoothly to point A, or from 0 to 5,000 psi. In the second stage of loading, an impact load $=1,000 \mathrm{psi}$, from $\mathrm{A}$ to $\mathrm{B}$, which is equivalent to breaking an overhanging roof of unit width with its length, thickness, and density equal to $55 \mathrm{ft}, 15 \mathrm{ft}$, and $175 \mathrm{lb} / \mathrm{ft}^{3}$, respectively. The impact load was applied for a very short period of time, $0.01 \mathrm{sec}$. It remains constant for $0.01 \mathrm{sec}$, from B to C. before dropping back to 5,000 psi in $0.01 \mathrm{sec}$ to point $\mathrm{D}$ from where the load remains at 5,000 psi for the remainder of the analysis. This proposed load history shown in Figure 4-3 is expected to simulate the situation of roof strata in the gob, where the load increases gradually with the progress of mining. Since the roof is very stiff and strong, it will not cave readily and it will overhang for a certain length. 
When the overhanging roof breaks, an impact load will be transmitted to the gateroad pillars underneath.

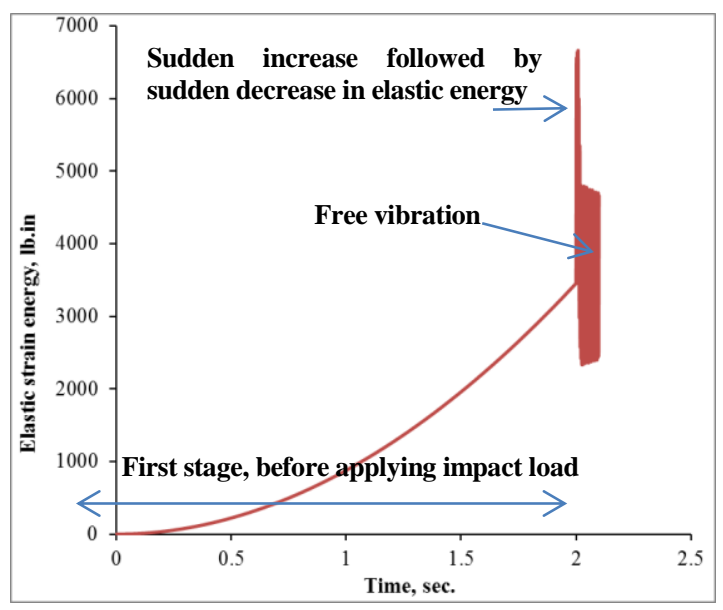

a

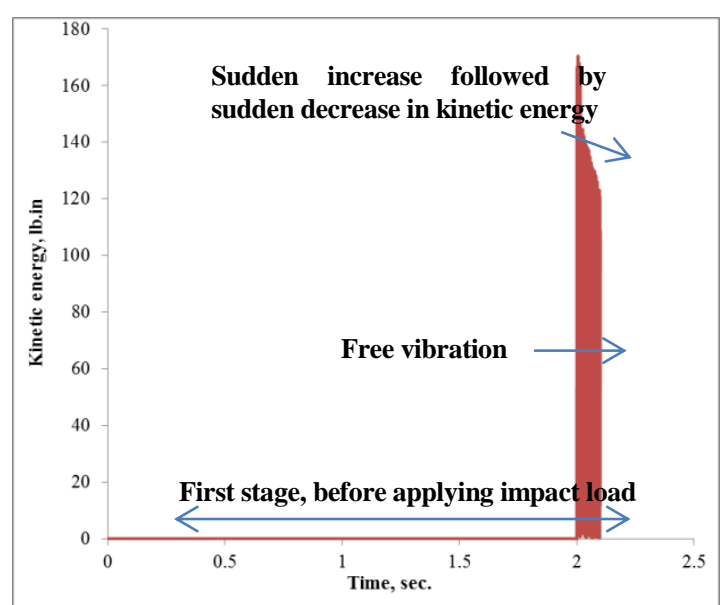

b

Figure 4-4 Variation of a) elastic strain energy and b) kinetic energy before and after applying the impact load

Figure 4-4 shows the variation of the elastic and kinetic energies with time. During the first stage of loading (static analysis) where the load is ramped up gradually, elastic energy increases gradually, while the kinetic energy is zero. In the second stage of loading, once the impact load is applied there was a sudden increase in the elastic energy, and once that impact load has been removed, both the kinetic energy and the elastic energy decrease instantaneously and so much energy is released that cracks initiated and or propagated inside the simulated coal specimen. Since the material damping has not been considered in the analysis, the coal particles vibrate freely around their equilibrium position.

As the work done in deforming an elastic material is the area beneath the stressstrain curve, it has been stored as elastic strain energy. When the stress is released the work is recovered and causes damage (Cook, 1963). In several instances of bumps, it was 
observed that unfilled cribs had been moved a number of feet laterally and left standing intact. This indicates a violent vibration of a strong immediate roof (Rice, 1935).

Figure 4-5 shows the variation of the vertical stress due to impact load at path 3-3. Once the coal specimen is subjected to the impact load, it experiences a sudden cyclic loading and unloading. The state of stress changes from state-0 (just before applying the impact load) to state-1 (Just after applying the impact load), then to state-2,....to state-5. Notice the direction of the arrows. All these events occur in a very short period of time. Such changes in the state of stress result in the generation and transmission of $(\mathrm{P} \& \mathrm{~S}$ waves) in the medium (Brady and Brown, 1999). By analogy with a coal pillar, these sudden loading and unloading events result in contraction and expansion of the pillar. If the pillar is loaded near its ultimate strength, the induced stress from the impact load might exceed the strength of the pillar and cause violent failures. It is expected that the intensity of the impulsive changes depends on the magnitude of the impact load, i.e., if the length and thickness of the overhanging roof are larger, the destructive effects of the impact load will be more significant. Rice (1935) observed two characteristic features of bumps over those pillars which had been crushed where the immediate roof is strong: 1) An open space was usually found between the crushed coal and the immediate roof and 2) The immediate roof is not broken. These observations refer to the hammering effect of the immediate roof. 


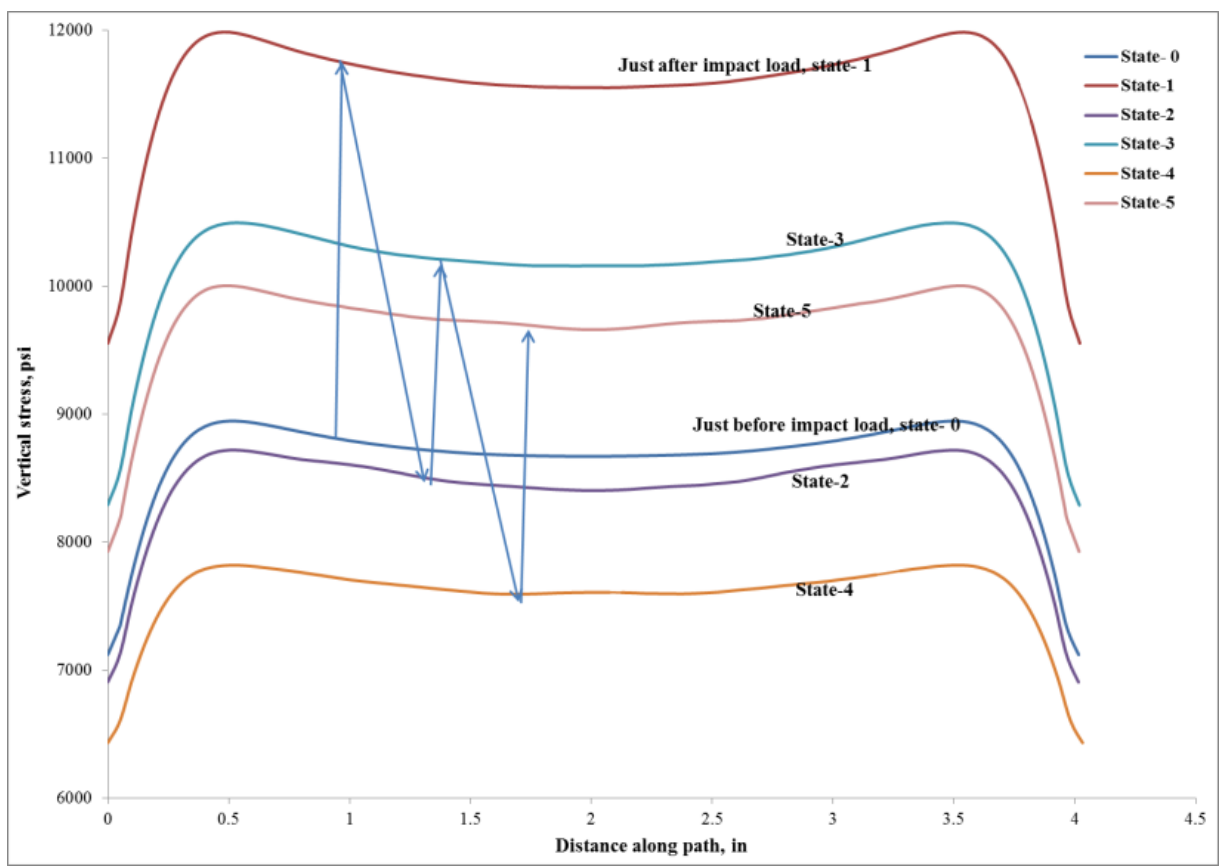

Figure 4-5 Variation of the vertical stress once the impact load was applied at path $3-3$

Therefore, a strong overlying stratum such as sandstone which occurs immediately above or close to the coal bed can cause bumps. Because such a stratum or strata does not cave readily and causes an impact load on the adjacent pillar when it breaks suddenly.

\subsection{Summary for Chapter Four}

Finite Element Modeling (FEM) for a simulated coal specimens showed that sudden changes in an interface friction or sudden breaking of the overhanging immediate roof is associated with sudden release of elastic and kinetic energies and a very rapid change in the vertical stress. These transient changes are expected to cause a violent failure if the coal specimens were already loaded close to their full bearing strength. According to Griffith (1921), the released energy is consumed in crack initiation and propagation. The kinetic energy is required to provide the edges with sufficient energy to be thrown away 
from the pillar. FEM demonstrated that both sudden impact loads and sudden changes in interface friction are associated with the sudden release of elastic energy and sudden increase of kinetic energy. 


\section{CHAPTER 5: THE ROLE OF INTERFACE FRICTION AND WIDTH-TO-HEIGHT RATIO ON THE FAILURE MODE OF COAL SPECIMENS}

\subsection{Introduction}

Bumps in coal mines have been recognized as a major hazard for many years. Previous studies showed that the violence of coal specimen failure depends on both the interface friction and width-to-height $(\mathrm{W} / \mathrm{H})$ ratio of a coal specimen.

The mode of failure for a uniaxially loaded coal specimen is a combination of both a shear failure along the interface and a compressive failure behavior in the coal. Unstable interface failures provide a sudden de-confinement to coal ribs, thus triggering unstable compressive failure in the sidewalls. The compressive failure of a coal specimen or a coal pillar can be controlled by changing its $\mathrm{W} / \mathrm{H}$ ratio. As the $\mathrm{W} / \mathrm{H}$ ratio increases, the ultimate strength increases. Hence, it is expected that with a proper combination of the interface friction and width-to-height $(\mathrm{W} / \mathrm{H})$ ratio of coal specimens, the mode of failure would change from a sudden violent failure, to a non-violent failure.

Little experimental work has been done to investigate how the interface friction and width-to-height $(\mathrm{W} / \mathrm{H})$ ratio of coal specimens would affect the violence of coal specimen failure. The main objective of chapter five is to determine at what $\mathrm{W} / \mathrm{H}$ ratio and interface friction the mode of failure changes from violent to non-violent. The prepared coal specimens in chapter five have width-to-height ratios ranging from 1 to 10 . These coal specimen were uniaxially tested under two interface frictions of 0.10 and 0.25 . 
First, the interface friction between the coal specimen and the loading platen was determined from the direct shear test. Second, the effect of interface friction and the $\mathrm{W} / \mathrm{H}$ ratio of coal specimen on the potential for violent failure were investigated.

\subsection{Determination of an Interface Friction}

The direct shear test was used to determine the interface frictions between coal specimen end surfaces and loading platen using the GCTS Rock Direct Shear System RDS200 (GCTS website). Two types of contacts were examined. The first type of contact was the greased coal/greased steel platens. The second type of contact was called "direct contact" where there is no grease between the coal specimen ends and steel platens. The Mohr-Coulomb envelopes were constructed from the test data performed under different normal stresses in order to determine the magnitude of the interface friction (please see Figure 5-1).

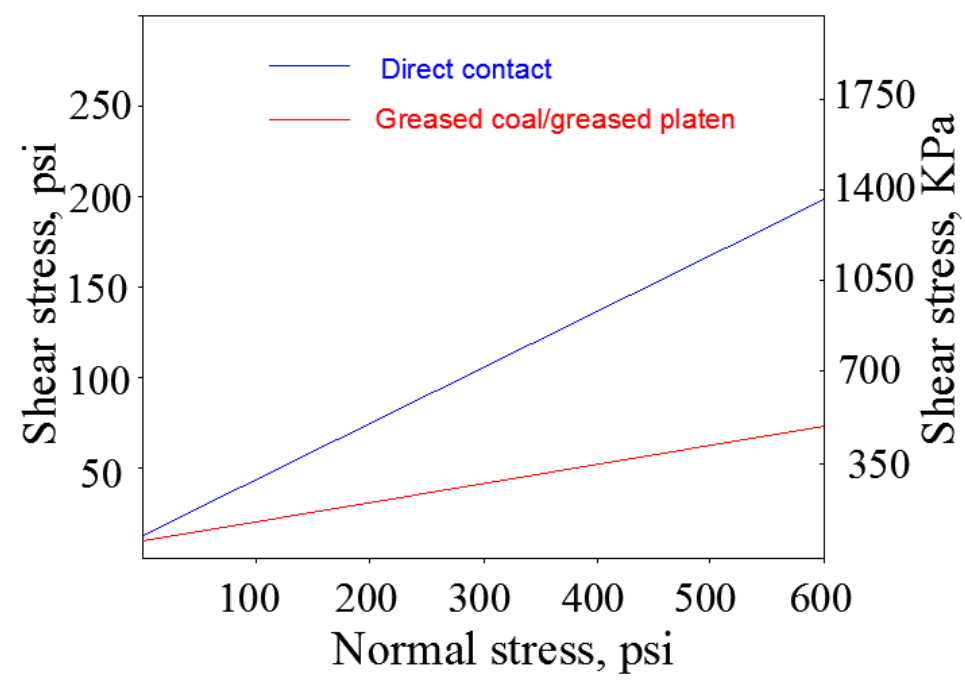

Figure 5-1 Mohr-Coulomb envelopes for interface friction between coal specimens and loading platens

As shown in Figure 5-1, when the end surfaces of coal specimen and the steel platens were lubricated with grease, the interface friction is approximately 0.10 , while the 
interface friction is approximately 0.25 for direct contact without grease. Table $5-1$ shows the test results for interface frictions 0.1 and 0.25 .

Table 5-1 Test results for 0.1 and 0.25 interfaces friction

\begin{tabular}{|c|c|c|}
\hline Interface friction & Normal stress, psi & Shear stress, psi \\
\hline \multirow{3}{*}{$\mu=0.1$} & 102.9 & 20.4 \\
\cline { 2 - 3 } & 202.9 & 50.6 \\
\cline { 2 - 3 } & 316.6 & 71 \\
\cline { 2 - 3 } & 422.8 & 80 \\
\hline \multirow{4}{*}{$\mu=0.25$} & 530.8 & 110 \\
\cline { 2 - 3 } & 104.3 & 80 \\
\cline { 2 - 3 } & 214.1 & 123 \\
\cline { 2 - 3 } & 321.8 & 150 \\
\cline { 2 - 3 } & 407.5 & 215.3 \\
\cline { 2 - 3 } & 537.6 & 285.1 \\
\hline
\end{tabular}

\subsection{Test Results and Discussion}

The NIOSH (Mine Roof Simulator) MRS was used to apply a load on coal specimens while a load cell was used to record the displacement of coal specimens to the applied load. No strain gauges were used to estimate the deformation of coal specimens. Hence, the load cell was used to record both the load and deformation of the coal specimens.

The MRS is the largest active load frame of its kind in the world. It was originally designed and still used for testing the structural integrity of longwall shields. It can accommodate specimens with dimensions up to 4.88 meters (16 feet) high, $6.1 \mathrm{~m} \mathrm{(20 \textrm {ft } )}$ wide and $6.1 \mathrm{~m}(20 \mathrm{ft})$ long. The MRS performs precision load testing by the closed-loop, 
servo controlled actuators with six degrees of freedom control of the lower platen. The MRS can apply up to 1,363.6 tons (3 million lbs) of vertical force through the 610-mm (24in) stroke of the lower platen. The reason for using the MRS machine for this research was that the ultimate strengths of some coal specimens, especially those specimens with large $\mathrm{W} / \mathrm{H}$ ratio are so large that it exceeds the ultimate capacity of WV laboratory testing machine. The $\mathrm{W} / \mathrm{H}$ ratio for the tested coal specimens ranged from 1 to 10 and the interface frictions were 0.10 and 0.25 . Figure 5-2 shows a coal specimen loaded by the MRS machine.

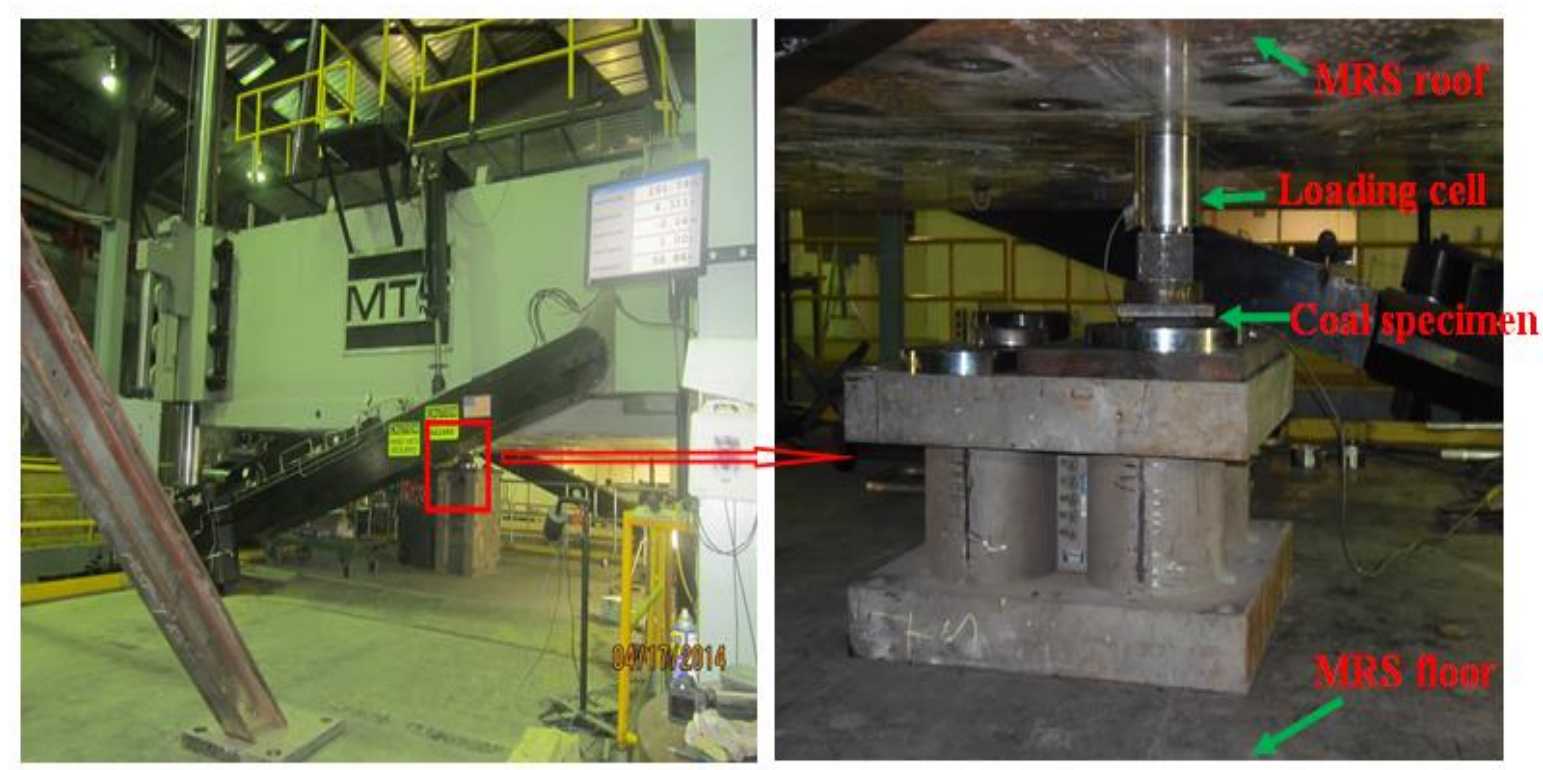

Figure 5-2 Coal specimen loaded by the NIOSH MRS Machine

The mode of failure, a violent or non-violent, has been examined for coal specimens having different $\mathrm{W} / \mathrm{H}$ ratio and interface friction. All tested coal specimens in chapter five are from the Sunnyside coal seam, Utah coal. The coal specimens have been tested under a load control of $320 \mathrm{lbf} / \mathrm{sec}$. Since the water content would affect the mechanical properties of coal, the coal specimens were air dried before testing. The 
average density for 17 cylindrical coal specimens of height to diameter ratio $=2$ is approximately $78.2 \mathrm{lb} / \mathrm{ft}^{3}$.

Coal specimens were divided into five different groups according to their mode of failure, end constraint conditions and $\mathrm{W} / \mathrm{H}$ ratio. Table 5-2 summarizes the test results for the five coal specimen groups while Table 5-3 summarizes the vertical stress test results for the five groups shown in Table 5-2.

Table 5-2 Mode of failure associated with coal specimens of different W/H ratios and end constraint conditions

\begin{tabular}{|c|c|c|c|c|c|}
\hline $\begin{array}{c}\text { Group } \\
\text { number }\end{array}$ & $\begin{array}{c}\text { W/H } \\
\text { ratio }\end{array}$ & $\begin{array}{c}\text { Interface } \\
\text { friction }\end{array}$ & $\begin{array}{c}\text { End contact } \\
\text { condition }\end{array}$ & $\begin{array}{c}\text { Number of } \\
\text { specimens }\end{array}$ & Mode of failure \\
\hline $\mathbf{1}$ & $1-3.8$ & 0.1 & $\begin{array}{c}\text { Greased } \\
\text { coal/greased } \\
\text { platens }\end{array}$ & 8 & $\begin{array}{c}\text { Failed suddenly but } \\
\text { not violently. }\end{array}$ \\
\hline $\mathbf{3}$ & $4.7-6.5$ & 0.1 & $\begin{array}{c}\text { Greased } \\
\text { coal/greased } \\
\text { platens }\end{array}$ & 8 & $\begin{array}{c}\text { Ductile failure with } \\
\text { clear plastic strain. }\end{array}$ \\
\hline $\mathbf{3}$ & $6.8-10$ & 0.1 & $\begin{array}{c}\text { Greased } \\
\text { coal/greased } \\
\text { platens }\end{array}$ & 6 & $\begin{array}{c}\text { Samples did not fail. } \\
\text { They were squeezed } \\
\text { badly and expanded } \\
\text { laterally. }\end{array}$ \\
\hline $\mathbf{5}$ & $1.2-3.6$ & 0.25 & $\begin{array}{c}\text { Direct contact } \\
\text { without grease }\end{array}$ & 13 & $\begin{array}{c}\text { Two specimens failed } \\
\text { very violently. Two } \\
\text { specimens failed non- } \\
\text { violently like group-1. } \\
\text { The others are slightly } \\
\text { more violent than } \\
\text { Group 1. }\end{array}$ \\
\hline & $4.8-8.5$ & 0.25 & $\begin{array}{c}\text { Direct contact } \\
\text { without grease }\end{array}$ & 22 & $\begin{array}{c}\text { Samples of W/H ratio } \\
\text { of 7.7 or more did not } \\
\text { fail. Samples less than } \\
7.7 \text { failed very } \\
\text { violently. }\end{array}$ \\
\hline
\end{tabular}


Table 5-3 Summary of vertical stress test results for the five groups shown in Table 5-2

\begin{tabular}{|c|c|c|c|c|c|c|}
\hline Group \# & Sample \# & $\begin{array}{c}\text { Length, } \\
\text { in }\end{array}$ & $\begin{array}{c}\text { Width, } \\
\text { in }\end{array}$ & $\begin{array}{c}\text { Height, } \\
\text { in }\end{array}$ & $\begin{array}{l}\text { W/H } \\
\text { ratio }\end{array}$ & $\begin{array}{c}\text { Vertical } \\
\text { stress, ksi }\end{array}$ \\
\hline \multirow{8}{*}{ Group \#1 } & 13 & 5.197 & 5.551 & 5.236 & 1.026 & 1.583 \\
\hline & 18 & 5.984 & 6.890 & 2.480 & 2.589 & 0.889 \\
\hline & 16 & 7.677 & 8.976 & 2.953 & 2.811 & 0.829 \\
\hline & 2 & 6.220 & 6.732 & 2.205 & 2.935 & 1.873 \\
\hline & 8 & 7.283 & 8.189 & 2.559 & 3.018 & 1.903 \\
\hline & 19 & 6.732 & 8.740 & 2.520 & 3.044 & 1.762 \\
\hline & 17 & 7.874 & 8.425 & 2.441 & 3.337 & 1.413 \\
\hline & 22 & 8.307 & 8.858 & 2.244 & 3.823 & 1.803 \\
\hline \multirow{8}{*}{ Group \#2 } & 30 & 5.709 & 5.551 & 1.189 & 4.735 & 1.588 \\
\hline & 68 & 5.039 & 5.315 & 0.996 & 5.196 & 1.718 \\
\hline & 34 & 5.236 & 5.630 & 1.008 & 5.387 & 2.784 \\
\hline & 49 & 7.283 & 7.677 & 1.339 & 5.586 & 2.631 \\
\hline & 37 & 5.945 & 5.787 & 1.043 & 5.622 & 2.630 \\
\hline & 59 & 6.850 & 7.165 & 1.165 & 6.012 & 2.913 \\
\hline & 43 & 7.244 & 7.047 & 1.142 & 6.258 & 2.645 \\
\hline & 61 & 7.283 & 7.756 & 1.169 & 6.428 & 3.007 \\
\hline \multirow{6}{*}{ Group \#3 } & 13 & 5.375 & 5.375 & 0.800 & 6.719 & 22.520 \\
\hline & 2 & 5.750 & 5.750 & 0.800 & 7.188 & 19.576 \\
\hline & 15 & 6.000 & 6.000 & 0.800 & 7.500 & 17.950 \\
\hline & 11 & 6.188 & 6.188 & 0.800 & 7.734 & 10.442 \\
\hline & 12 & 6.500 & 6.500 & 0.800 & 8.125 & 15.382 \\
\hline & 17 & 5.000 & 5.000 & 0.500 & 10.000 & 26.359 \\
\hline Group \#4 & 14 & 5.827 & 6.142 & 4.803 & 1.245 & 3.358 \\
\hline
\end{tabular}




\begin{tabular}{|c|c|c|c|c|c|c|}
\hline & 5 & 7.283 & 6.693 & 4.370 & 1.598 & 3.252 \\
\hline & 12 & 6.732 & 6.457 & 3.780 & 1.744 & 3.054 \\
\hline & 3 & 7.402 & 7.480 & 3.600 & 2.067 & 3.550 \\
\hline & 1 & 5.906 & 5.630 & 2.677 & 2.154 & 2.596 \\
\hline & 10 & 5.354 & 6.299 & 2.480 & 2.341 & 6.329 \\
\hline & 21 & 6.339 & 5.551 & 2.323 & 2.554 & 2.726 \\
\hline & 6 & 6.772 & 6.693 & 2.598 & 2.591 & 5.842 \\
\hline & 20 & 5.827 & 6.102 & 2.205 & 2.705 & 5.389 \\
\hline & 9 & 5.276 & 5.315 & 1.890 & 2.802 & 5.918 \\
\hline & 4 & 8.701 & 8.071 & 2.953 & 2.838 & 4.723 \\
\hline & 7 & 7.008 & 7.087 & 2.300 & 3.064 & 4.696 \\
\hline & 15- reload & 8.740 & 8.583 & 2.402 & 3.606 & 4.387 \\
\hline \multirow{16}{*}{ Group \#5 } & 51 & 6.890 & 7.165 & 1.441 & 4.876 & 8.500 \\
\hline & 33 & 5.276 & 5.315 & 1.004 & 5.274 & 9.062 \\
\hline & 32 & 6.299 & 6.417 & 1.154 & 5.512 & 8.273 \\
\hline & 39 & 6.024 & 6.378 & 1.106 & 5.603 & 8.499 \\
\hline & 42 & 6.220 & 6.378 & 1.079 & 5.839 & 10.088 \\
\hline & 66 & 7.677 & 8.031 & 1.283 & 6.118 & 8.473 \\
\hline & 53 & 7.283 & 7.480 & 1.197 & 6.167 & 9.660 \\
\hline & 1 & 5.063 & 5.063 & 0.800 & 6.328 & 7.720 \\
\hline & 40 & 6.496 & 6.575 & 1.028 & 6.360 & 11.028 \\
\hline & 55 & 6.693 & 6.693 & 1.047 & 6.391 & 11.300 \\
\hline & 60 & 7.402 & 7.402 & 1.130 & 6.551 & 11.844 \\
\hline & 7 & 5.563 & 5.563 & 0.800 & 6.953 & 8.347 \\
\hline & 36 & 6.181 & 6.614 & 0.913 & 7.000 & 9.625 \\
\hline & 6 & 5.750 & 5.625 & 0.800 & 7.109 & 16.492 \\
\hline & 57 & 7.480 & 7.677 & 1.059 & 7.156 & 10.088 \\
\hline & 3 & 6.000 & 6.000 & 0.800 & 7.500 & 15.844 \\
\hline
\end{tabular}




\begin{tabular}{|c|c|c|c|c|c|c|}
\hline 14 & 6.000 & 6.250 & 0.800 & 7.655 & 17.346 \\
\cline { 2 - 7 } & 8 & 6.000 & 6.250 & 0.800 & 7.655 & 17.225 \\
\cline { 2 - 7 } & 4 & 6.125 & 6.125 & 0.800 & 7.656 & 17.437 \\
\hline 47 & 7.283 & 7.087 & 0.917 & 7.832 & 17.100 \\
\hline & 62 & 7.441 & 7.244 & 0.925 & 7.935 & 17.500 \\
\hline & 58 & 8.268 & 8.031 & 0.957 & 8.518 & 17.000 \\
\hline
\end{tabular}

\subsection{Detailed Analysis of the Results for Each Group}

\subsubsection{Group 1}

The interface friction was 0.1 and the $\mathrm{W} / \mathrm{H}$ ratio ranged from 1 to 3.8 . The failure of coal specimens in group 1 was sudden but non-violent. Once the ultimate strength was reached, sudden loss of strength occurred. It was accompanied by very low acoustic emissions. Debris ejections from the edges of the coal samples are few and at low speed, such that the failure of the coal specimen in group 1 was not recognized until the machine stopped automatically when coal specimen stopped to provide anymore resistance to the applied load.

Figure 5-3 shows an example of crushing failure for coal specimen \#13 in group 1. It had a cross-sectional dimensions of $132.1 \times 142.2 \mathrm{~mm}(5.2 \times 5.6 \mathrm{in})$ and a height of $132.1 \mathrm{~mm}$ (5.2 in), making the $\mathrm{W} / \mathrm{H}$ ratio approximately 1 . With small $\mathrm{W} / \mathrm{H}$ ratio and a low end confinement, the specimen was crushed and ejected with little remnant core between the platens. The debris sizes for the rib and core zones were similar. 


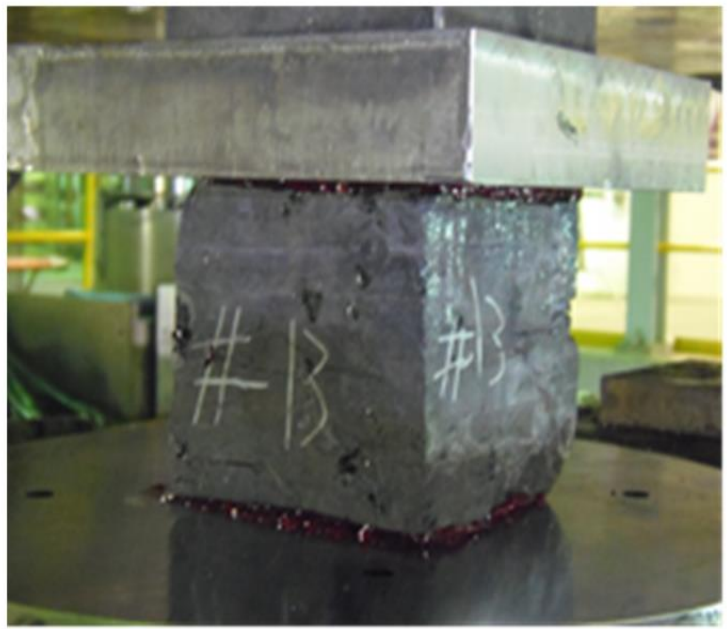

Specimen \# 13 before failure

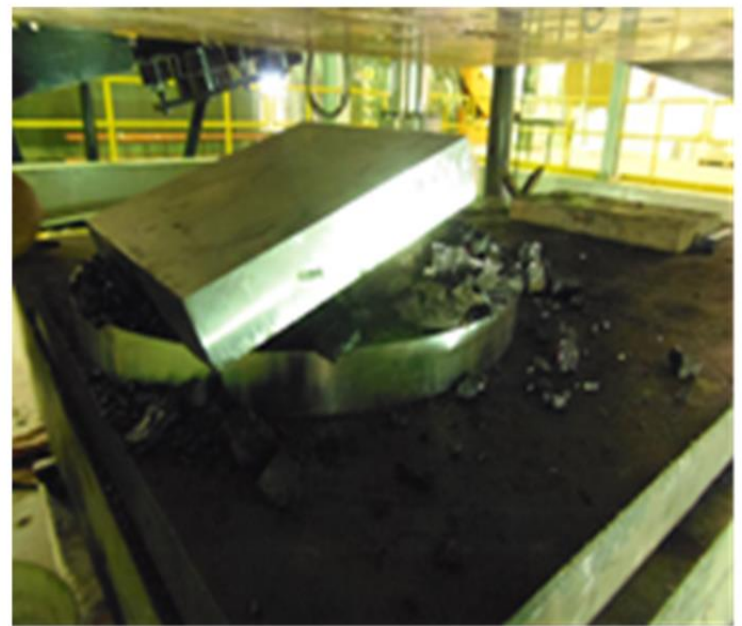

Specimen \# 13 after failure

Figure 5-3 Coal specimen No. 13 in group 1 of W/H ratio= 1 before and after failure

Figure 5-4 shows an example of squeezing failure for coal specimen \#17 in group 1. It had a cross-sectional dimensions of $200.7 \times 213.4 \mathrm{~mm}(7.9 \times 8.4$ in) and a height of 71 $\mathrm{mm}$ (2.4 in), making the $\mathrm{W} / \mathrm{H}$ ratio approximately 3.3 . By comparing the deformed shape for specimens \# 13 and \# 17 whose W/H ratios are approximately 1 and 3.3 respectively, for specimen \#13, the core of which was crushed and damaged, whereas the core of specimen \#17, shown in Figure 5-4, was squeezed and expanded laterally while the ribs were crushed. Neither splitting nor fault planes were observed in the core of the failed specimen \#17; however, it was disintegrated and damaged, probably by shear failure. In other words, the structural integrity of the core was lost after testing. Hence, for coal specimens in group-1, the $\mathrm{W} / \mathrm{H}$ ratio determines the deformed shape of the failed coal specimens whether crushing or squeezing. 

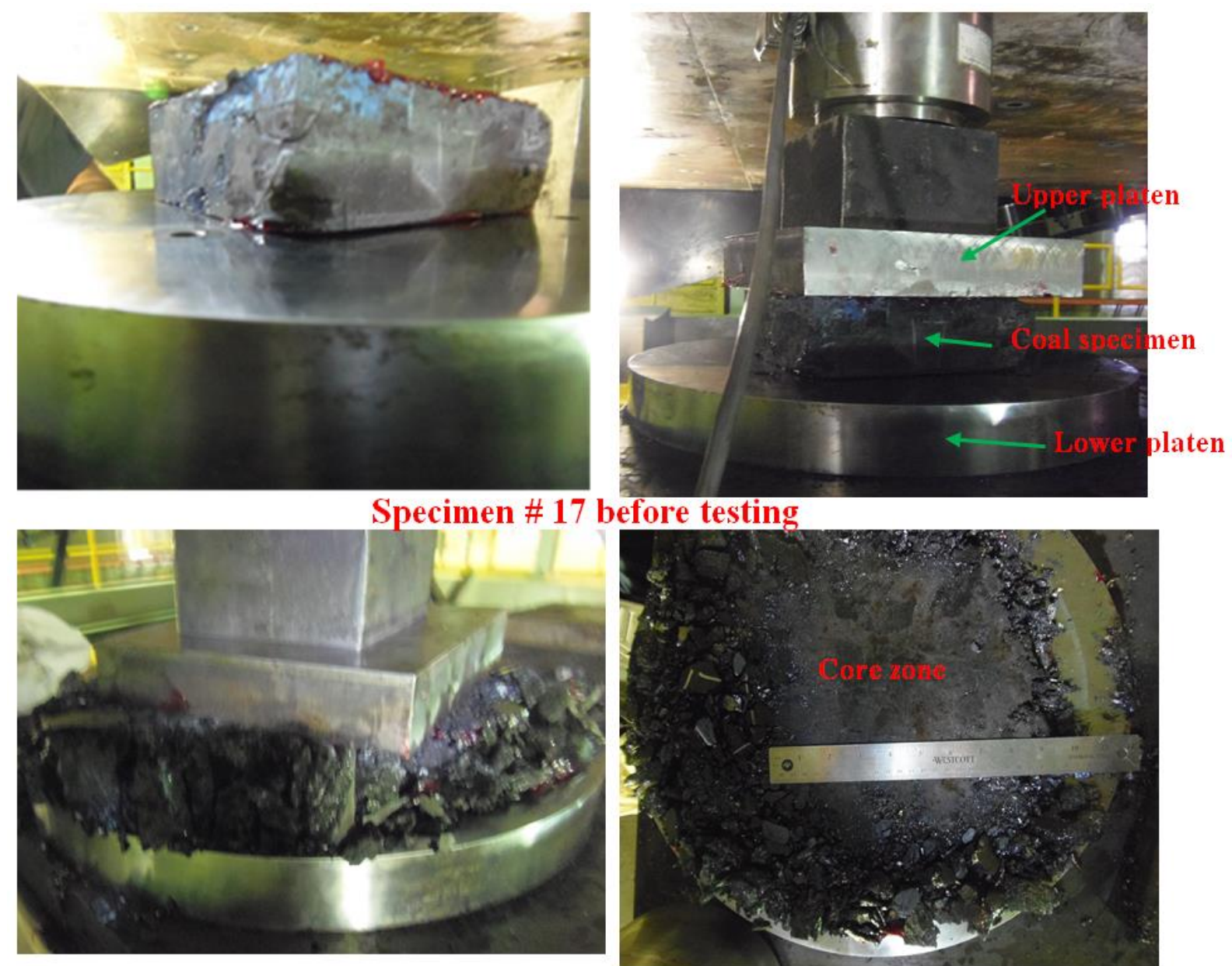

\section{efore testing}

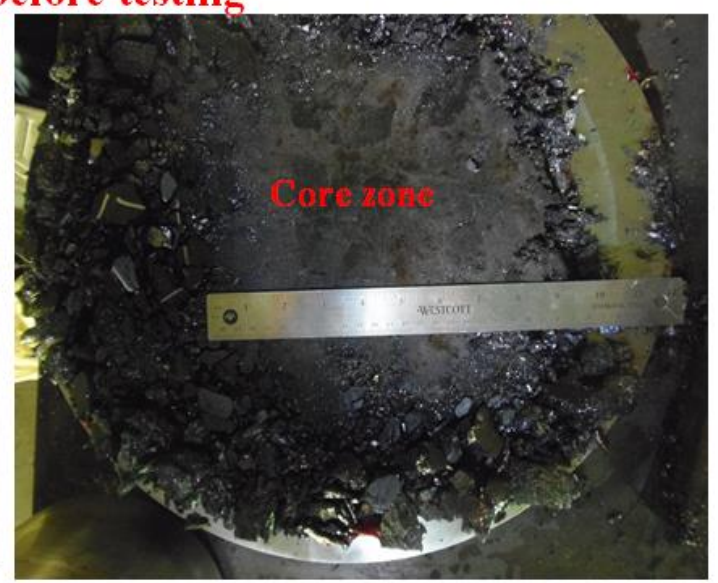

\section{Specimen \# 17 after testing}

Figure 5-4 Coal specimen No. 17 in group 1 with $\mathrm{W} / \mathrm{H}$ ratio $=3.3$ before and after failure

For coal specimens tested in the laboratory there are two sources of end confinement; the first one is the $\mathrm{W} / \mathrm{H}$ ratio and the second one is the interface friction between the machine platens and the coal specimen. For specimen \#13, both the interface friction and $\mathrm{W} / \mathrm{H}$ ratio were small; this was why it was crushed completely upon failure. For coal specimen $\# 17$, the interface friction was low, but the $\mathrm{W} / \mathrm{H}$ ratio was relatively high as compared to specimen \#13. This was why specimen \#17 was squeezed and expanded upon failure. Note that squeezed coal specimen has a clear core. However, crushed coal 
specimen does not have a core and the particle size distribution for both the core zone and rib zone is relative close.

Figure 5-5 shows typical stress-strain curves obtained for three coal specimens in group 1. The other coal specimens in group 1 exhibited the same behavior. It is obvious that the mode of failure is characterized by brittle failure with strain softening. A sudden loss of strength occurs and the strength decreases with increasing strain until the residual strength is reached.

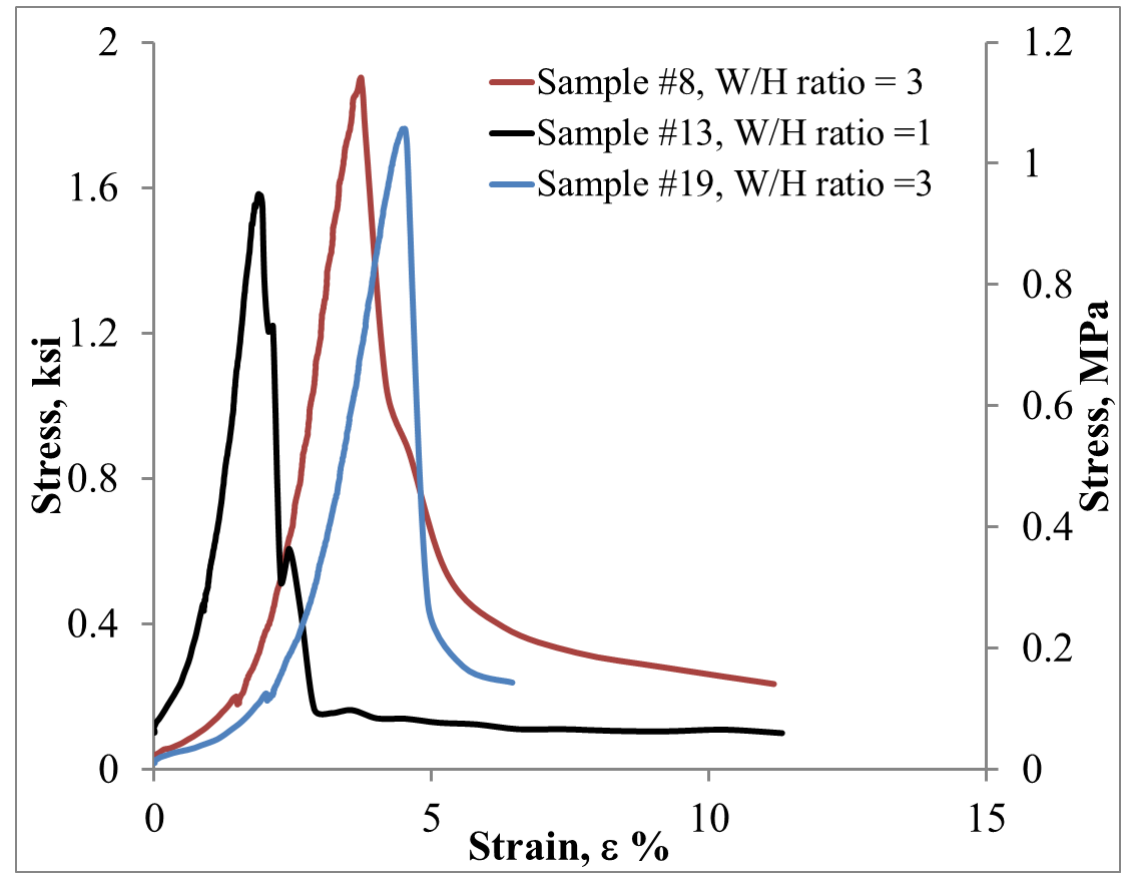

Figure 5-5 Stress-strain curves for three specimens of different W/H ratios in group 1

Therefore, a low interface friction between coal specimens and machine platens does not prevent sudden failure when the $\mathrm{W} / \mathrm{H}$ ratio of coal specimens is as small as that shown in group 1. However, it reduces the degree of violence in terms of noise and ejection, e.g. low noise pitch and low debris ejections at failure, unfortunately no acoustic 
emission equipment has been used, so the noise pitch and ejections are visual finding. Coal specimens in group 1 were either crushed or squeezed, depending on the $\mathrm{W} / \mathrm{H}$ ratio.

\subsubsection{Group 2}

The interface friction was 0.1 and the $\mathrm{W} / \mathrm{H}$ ratio ranged from 4.7 to 6.5 . Deformations of the specimens became more ductile than those in group 1, because the coal specimens could sustain more permanent deformation without sudden loss in load carrying capacity due to the confinement provided with high $\mathrm{W} / \mathrm{H}$ ratio. On the contrary, for group 1, brittle failure took place with no or very little plastic strain. Figure 5-6 shows the stress-strain curve for coal specimen \#30 in group 2. It had cross-sectional dimensions of $144.8 \times 142.2 \mathrm{~mm}(5.7 \times 5.6 \mathrm{in})$ and a height of $27.9 \mathrm{~mm}(1.1 \mathrm{in})$, making the $\mathrm{W} / \mathrm{H}$ ratio $=4.7$. Note that the other coal specimens in group 2 exhibited the same behavior.

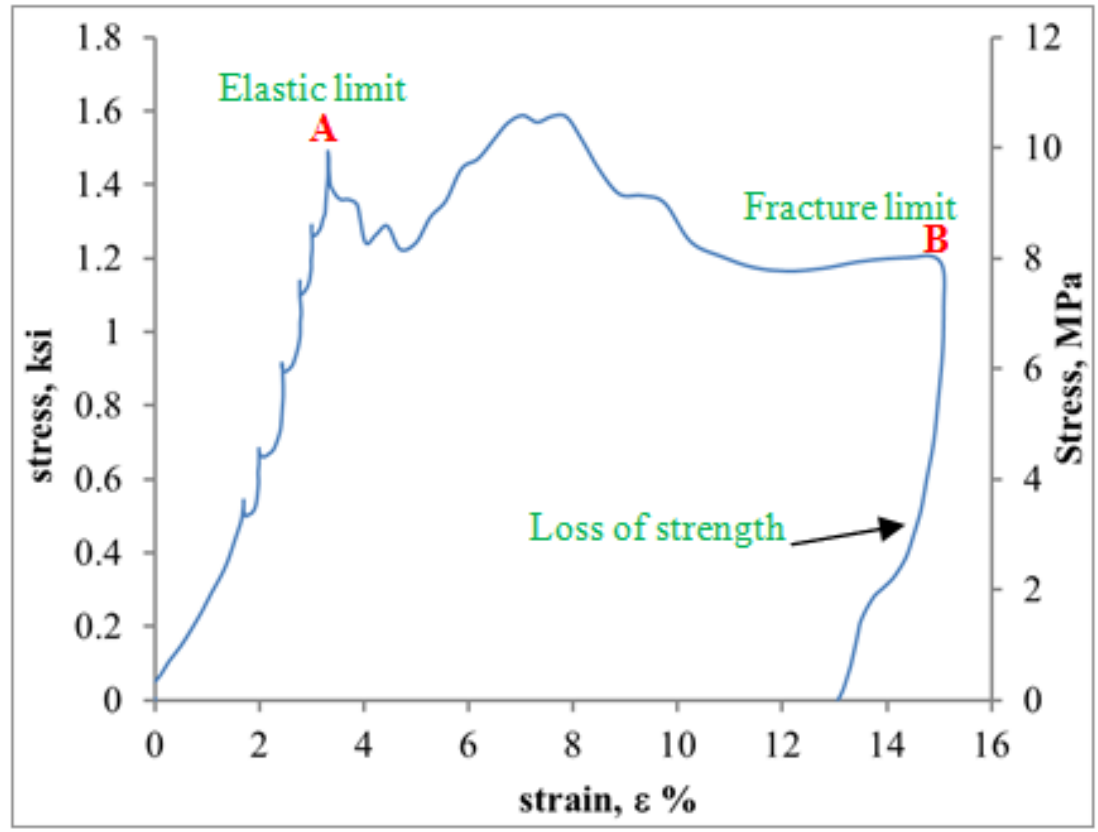

Figure 5-6 Stress-strain curve for a specimen No. 30 of $\mathrm{W} / \mathrm{H}$ ratio $=4.7$ in group 2 
The difference in the mode of failure between coal specimens in group 1 and group 2 can be recognized by comparing Figures 5-5 and 5-6. Point A in Figure 5-6 represents the elastic limit, which is the beginning of the plastic strain, while point B represents the fracture limit. The plastic strain sustained is more than three times the elastic strain. The other coal specimens in group 2 exhibited similar behavior.

Therefore, for an interface friction of 0.1 , brittle-ductile transition occurred when the $\mathrm{W} / \mathrm{H}$ ratio changed from group $1(\mathrm{~W} / \mathrm{H}$ ratio $=1-3.8)$ to group $2(\mathrm{~W} / \mathrm{H}$ ratio $=4.7-6.5)$. In general, the coal specimens remained brittle when the $\mathrm{W} / \mathrm{H}$ ratio was less than or equal to 3.8 ; on the other hand, when the $\mathrm{W} / \mathrm{H}$ ratio ranged from 4.7 to 6.5 , the coal specimens remained ductile, under which the coal specimen could sustain further permanent deformation without sudden failure.

Figure 5-7 shows a representative example for a coal specimen, i.e., specimen \#30 in group 2. The failed coal specimen experienced uniform stress distribution because the damage was almost the same everywhere through the coal specimen, except near the corners that suffered more damage. The grease on the top and the bottom surfaces of the coal specimen helped both the edges and the center to deform laterally with almost the same magnitude. Similar modes of failures were found for the other coal specimens in group 2. 


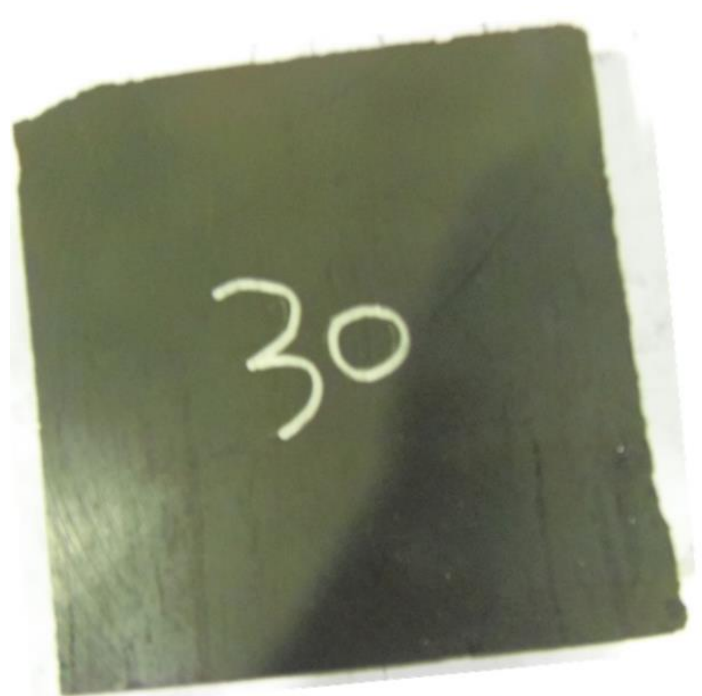

Before testing

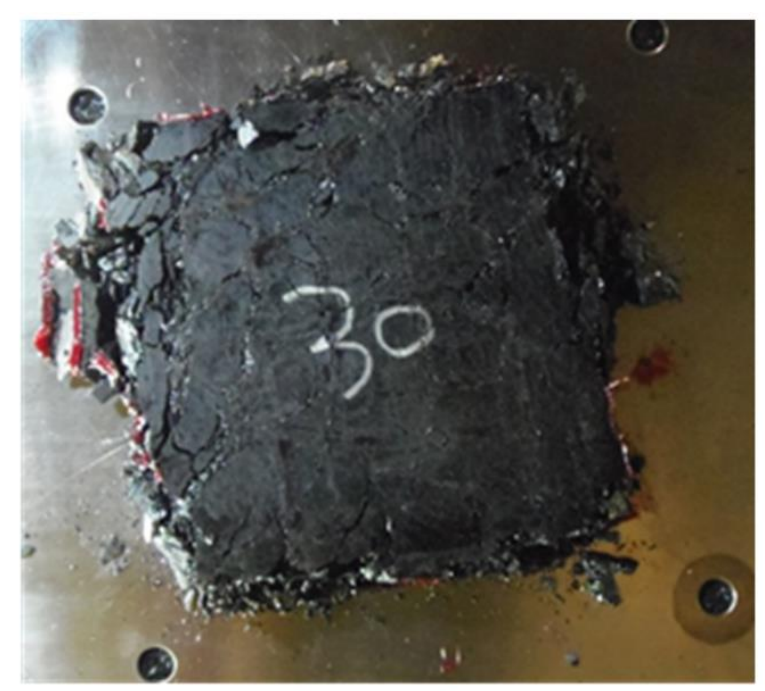

After testing

Figure 5-7 Coal specimen No. 30 in group 2 before and after failure

\subsubsection{Group 3}

The interface friction was 0.1 and the $\mathrm{W} / \mathrm{H}$ ratio was more than 6.7. The failure of coal specimens in group 3 was very stable, i.e. it was neither sudden nor violent failure. The debris ejections and the noise were very low; moreover, no loss of strength occurred. Coal specimens in group 3 were squeezed and expanded laterally such that their final crosssectional areas were obviously larger than their initial ones. All tests were stopped at an ultimate load of $2,891 \mathrm{KN}(650,000 \mathrm{lbs})$ or $138+\mathrm{MPa}(20+\mathrm{ksi})$. It was believed that increasing the load to more than $2,891 \mathrm{KN}$ (650 kips) will not change the mode of failure of coal specimens in group 3; these coal specimens have already experienced very high percentage strains, meaning that they have been subjected to more compaction and pulverization due to increasing load. Therefore, the tests were stopped at that load value.

Figure 5-8 shows a coal specimen in group 3 before and after testing. Specimen \#11 had cross-sectional dimensions of 157.5 x $157.5 \mathrm{~mm}(6.2 \times 6.2 \mathrm{in})$ and a height of $20.3 \mathrm{~mm}$ (0.8 in), making the $\mathrm{W} / \mathrm{H}=7.8$. The grease on the top and the bottom end surfaces of coal 
specimen helps the coal specimen to expand. A proper combination of $\mathrm{W} / \mathrm{H}$ ratio and interface friction helped lessen the elastic energy stored in the central portion of coal specimen and allowed the coal specimen to fail smoothly. As shown in Figure 8, almost no debris was ejected from the edges of coal specimen.

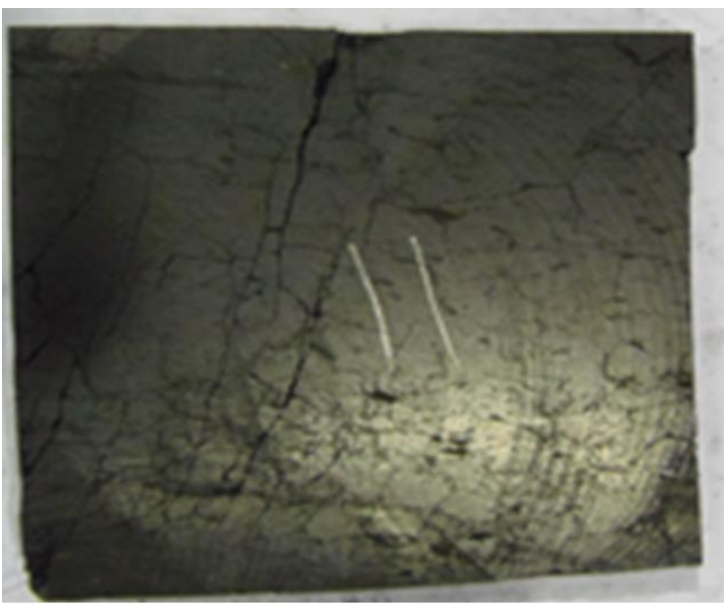

Specimen \# 11 before testing

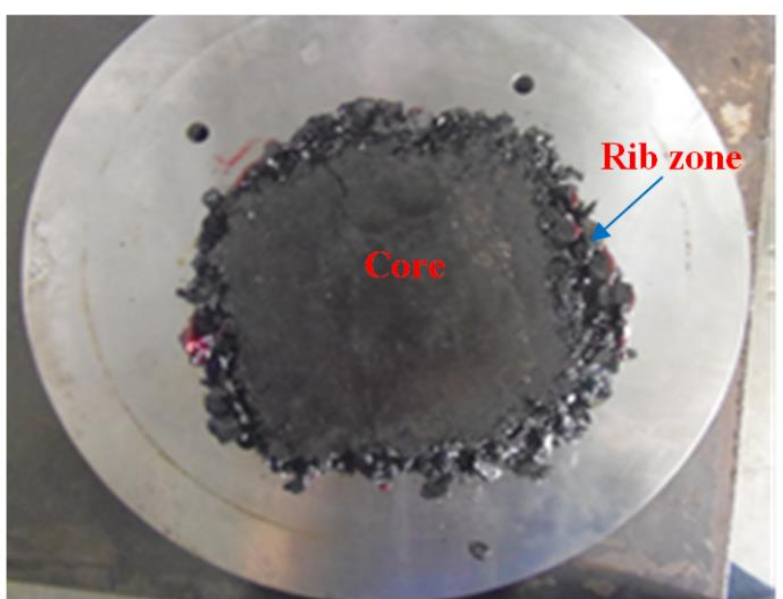

Specimen \# 11 after testing

Figure 5-8 Coal specimen No. 11 in group 3 before and after failure

The central portion of the coal specimen is known as the core zone (Morsy, 2003). The mechanical load applied on a coal specimen is transferred into elastic and plastic energies, with the plastic energy dissipated through crack initiation and propagation in the rib zone while most of the elastic energy is stored in the core zone (Prassetyo et.al. 2011). It was found that when the $\mathrm{W} / \mathrm{H}$ ratio is greater than or equal to 6.7 and the interface friction is 0.10 , the elastic strain energy, stored in the core zone that could manifest itself as a coal bump lessened, ultimately changing the mode of failure to a stable non-sudden, non-violent failure.

The typical loading-unloading stress-strain curve for a coal specimen in group 3 is shown in Figure 5-9. It is divided into three segments. The first segment from 0 to A represents a linear relationship between the stress and strain. The second segment from A 
to $\mathrm{B}$ represents a linear strain hardening, while the third segment from $\mathrm{B}$ to $\mathrm{C}$ represents a parabolic strain hardening. There is a considerable increase in the cross-sectional area of the coal specimen in the third segment due to expansion and squeezing. In Figure 5-9, the stress was determined based on the original cross-sectional area of the coal specimens. This was why the third segment of the stress-strain curve was characterized by a parabolic strain hardening. For coal specimen \# 17 whose stress-strain curve is shown in Figure 5-9, its initial cross-sectional area was 5 in $\mathrm{X} 5$ in, while its final cross-sectional area was approximately 7.1 in X 7.2 in.

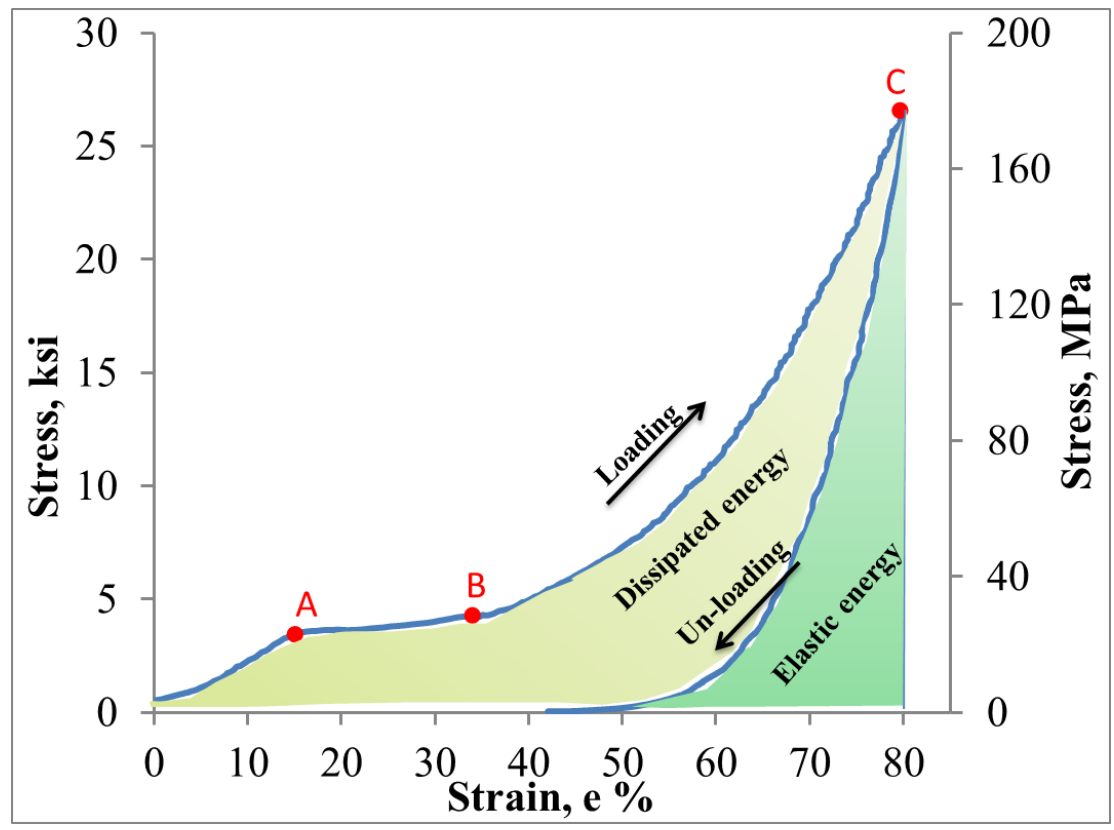

Figure 5-8 A typical loading-unloading stress-strain curve for coal specimen in group 3

As shown in Figure 5-9, the elastic energy is smaller than the dissipated energy. Hence, the burst index would be smaller than 1.0, which means that the coal specimen is very unlikely to bump. This demonstrates that coal specimens with a $\mathrm{W} / \mathrm{H}$ ratio of 6.7 or more and interface friction of 0.1 would experience smooth failure, where the elastic energy stored in the core released smoothly. The burst index is determined from the elastic 
hysteresis loop in the uniaxial compression loading and unloading tests on rock specimens, up to approximately $80 \%$ of the compressive strength. The burst index is defined as the ratio of the strain energy retained (the elastic energy) to the strain energy dissipated (the plastic energy). Higher \% strain associated with strain hardening behavior indicate that most of the coal specimen is being pulverized.

Gu and Ozbay (2014) used distinct element numerical modeling to investigate the unstable failures in underground mining conditions. They found that when the interfaces experience a stable slip failure, coal sidewalls are more likely to fail in a stable manner. With occurrence of unstable slips at interfaces, unstable compressive failures are triggered. By combining the test results of group $3 \& \mathrm{Gu}$ and Ozbay's conclusion, it is obvious that coal specimens in group 3 experienced stable slip failure, thus stable compressive failure.

It was found that, at a specific combination of $\mathrm{W} / \mathrm{H}$ ratio and interface friction, the failure becomes stable. So the stable failure along the interface is a function of both interface friction and $\mathrm{W} / \mathrm{H}$ ratio. A stable slip failure at the interface prompted the coal specimen to fail smoothly and gradually, and therefore, the elastic energy stored in the core lessened gradually as the core deteriorated gradually with increasing vertical load. Hence, according to the test results in this research, a coal specimen with as small interface friction of 0.1 and $\mathrm{W} / \mathrm{H}$ ratio of 6.8 or greater is expected to fail neither suddenly nor violently because this combination of interface friction and $\mathrm{W} / \mathrm{H}$ ratio allows the interface to fail smoothly and gradually, not suddenly, resulting in no sudden loss of load-carrying capacity for the coal specimens. 


\subsubsection{Group 4}

The $\mathrm{W} / \mathrm{H}$ ratio of the coal specimens ranged from 1.2 to 3.6 and the interface friction was 0.25 . Two coal specimens of a total of 13 failed suddenly, but not violently, with low noise and debris ejection. Conversely, the remaining 11 coal specimens failed very violently with high debris ejection and high-pitched noises. In general, the failure of coal specimens in group 4 was more violent in terms of noise and debris ejection than the other groups 1-3.

The two coal specimens in group 4 that did not fail violently, specimens \#1 and \#21, had the lowest vertical stress among the 13 tested coal specimens in that group. The term "vertical stress" is used rather than the UCS (uniaxial compressive strength) because the width-to-height ratio of the specimens is higher than 0.5 . Specimen \#21 had the lowest Young's modulus. The elastic energy stored in a uniaxially loaded coal specimen is directly proportional to the square of the UCS (Obert and Duvall, 1967), which means that the potential for violent failure increases with increasing UCS. Specimen \#1 did not have any visible cracks, while specimen \#21 has a crack at its center, but it did not affect its structural integrity, because the coal specimen was one piece.

Figure 5-10a \& 5-10b show the result of variation of the vertical stress and Young's modulus for coal specimens of the same range of $\mathrm{W} / \mathrm{H}$ ratio but different interface friction. To be specific, the comparison includes coal specimens in group 1 and group 4, where the $\mathrm{W} / \mathrm{H}$ ratio ranges from 1 to 3.8 while the interface frictions are 0.1 and 0.25 for group 1 and group 4, respectively. It is obvious that the interface friction has significant influence on the vertical stress and Young's modulus of the coal specimens. The Figures also show that both the vertical stress and the Young's modulus decrease with decrease in the interface friction. 
Hence, increasing the interface friction provides the coal specimen with supplemental strength.

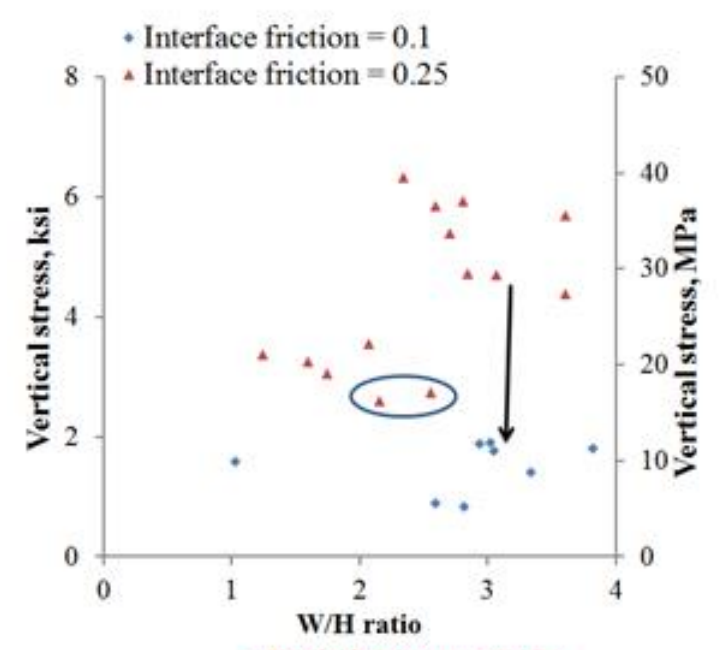

a. Vertical stress test results

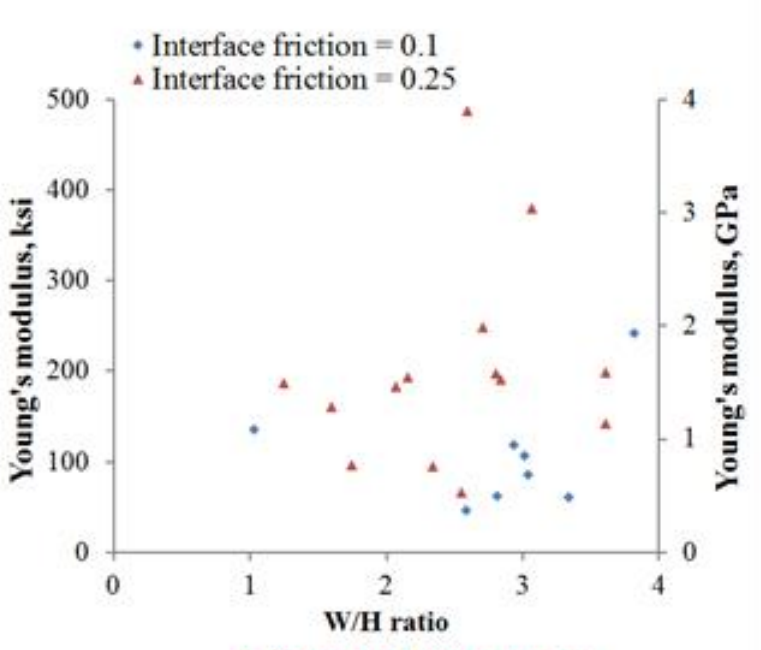

b. Young's modulus test results

Figure 5-9 Variation of (a) the vertical stress and (b) Young's modulus with the same range of $\mathrm{W} / \mathrm{H}$ ratio and different interface frictions

The two data points inside the ellipse shown in Figure 5-10a are for specimens \#1 and \#21, which did not fail violently, are unlike the other coal specimens in group 4 . These two coal specimens have the minimum vertical stress among coal specimens in group 4 with interface friction equaling 0.25 . Moreover, their vertical stress values are very close to the coal specimens in group 1 with interface friction of 0.1 . This gives a likely indication that an exceptionally low strength coal pillar would not fail violently in terms of noise and ejection. Sudden reduction and/or loss of constraint might cause bumps.

Figure 5-10.a has two groups of specimens of same W/H ratio, however different interface friction. For coal specimens of interface friction 0.25 , the vertical stress of a coal specimen with $\mathrm{W} / \mathrm{H}$ ratio $=1-3.8$ would be one of the red triangles shown in Figure 5-10a. 
At a specific $\mathrm{W} / \mathrm{H}$ ratio, for instance 3 or 3.6 , assuming further that the initial interface friction is suddenly changed from 0.25 to 0.1 , its vertical stress would suddenly decrease from the levels of red triangles to those of the blue diamonds as shown by the vertical black arrows in Figure 5-10a, and the coal specimen or coal pillar may fail violently. Hence, the failure of interface contact will trigger the compressive failure of a coal specimen, and therefore coal bumps could be avoided when a proper combination of interface friction and $\mathrm{W} / \mathrm{H}$ ratio exists.

Figure 5-11 shows an example of crushing failure for specimen \#14 in group 4 that failed violently where the pitch of noise and the debris ejections from the ribs were high. It had a cross-sectional dimension of $147.3 \times 154.9 \mathrm{~mm}(5.8 \times 6.1 \mathrm{in})$ and a height of 121.9 $\mathrm{mm}$ (4.8 in) with a $\mathrm{W} / \mathrm{H}=1.2$. It was crushed completely without core upon failure.

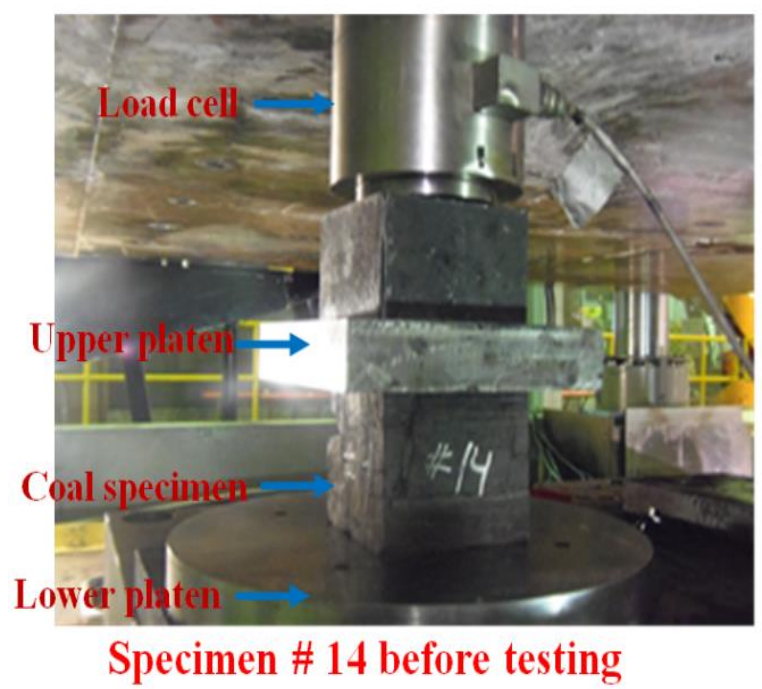

Figure 5-10 Coal specimen No. 14 in group 4 of $\mathrm{W} / \mathrm{H}$ ratio $=1.2$ before and after failure

Figure 5-12 shows specimen \#15 in group 4; it had a cross-sectional dimension of $221 \times 218.4 \mathrm{~mm}(8.7 \times 8.6 \mathrm{in})$ and a height of $61 \mathrm{~mm}(2.4 \mathrm{in})$ with a $\mathrm{W} / \mathrm{H}=3.6$. It was loaded to its ultimate strength. Once the peak strength was reached, the load was released 
and the coal specimen was examined. No damage was found at the center; however, little damage occurred at the upper left corner of the coal specimen (visual finding). Although the specimen was loaded close to its ultimate strength, it is still structurally intact (see Figure 5-12).

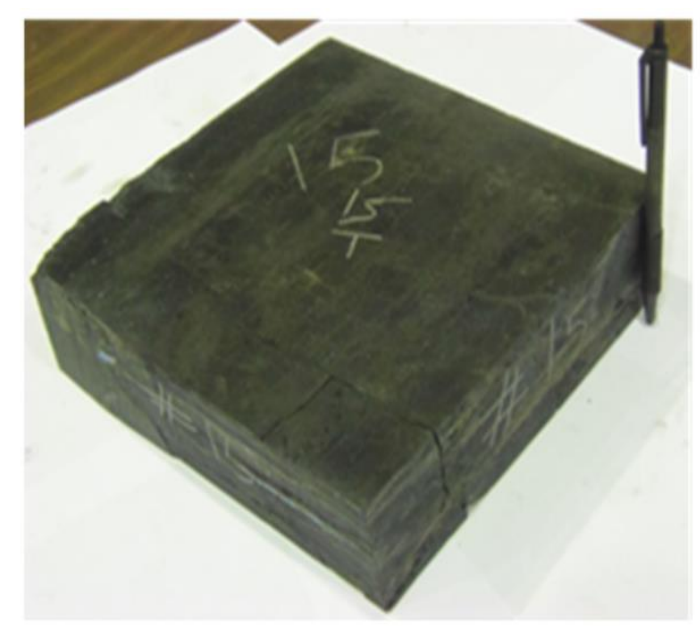

Sample \# 15 before testing ( $\left(1^{\text {st }}\right.$ loading)

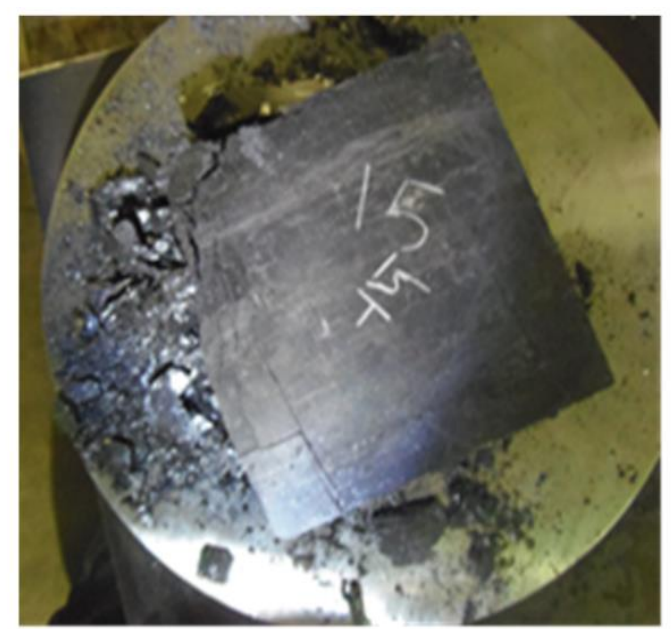

Sample \# 15 after testing ( $1^{\text {st }}$ loading)

Figure 5-11 Coal specimen No. 15. It was loaded to its ultimate strength and then the load was removed quickly

This coal specimen was expected to fail violently if the load was not removed, because the elastic energy stored in it was almost three times the dissipated energy as shown in Figure $5-13$. 


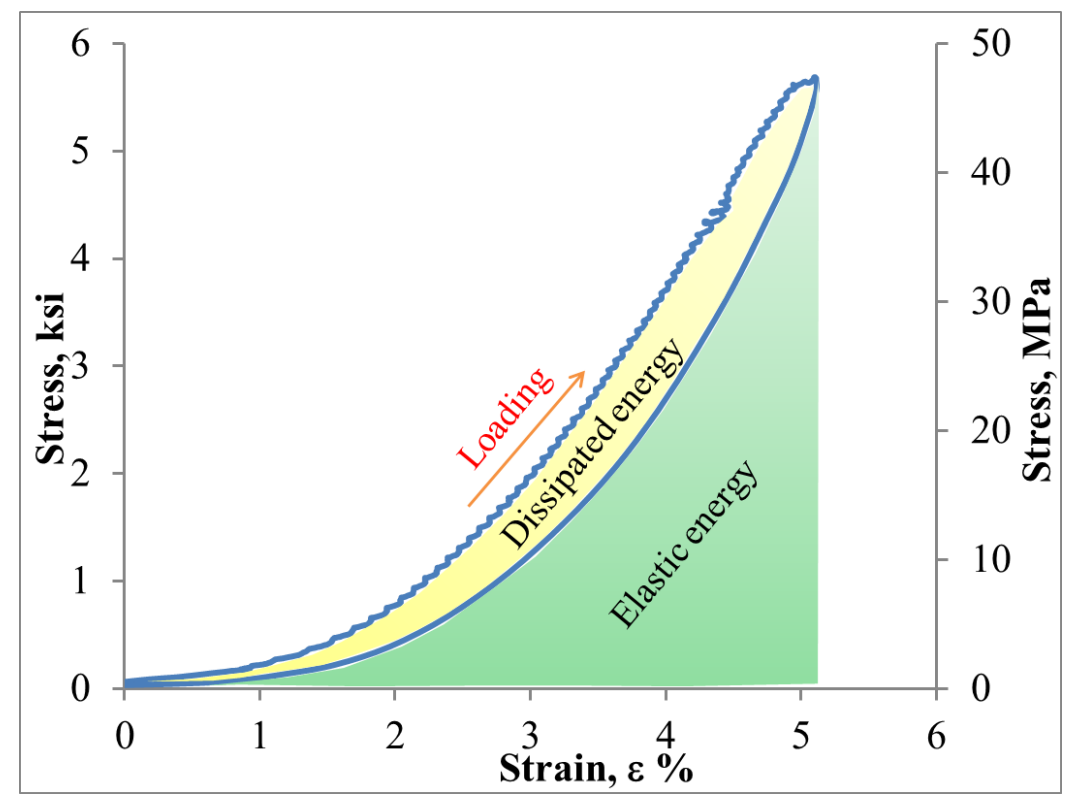

Figure 5-12 Loading-unloading stress-strain curve of specimen No. 15 in group 4

After complete unloading, specimen \#15 was reloaded again until it failed. The failure during the reloading stage was sudden and very violent in terms of noise and debris ejections. Examination of the failed specimen showed that the core was not intact, but highly deteriorated. The ribs were completely damaged, as shown in Figure 5-14.

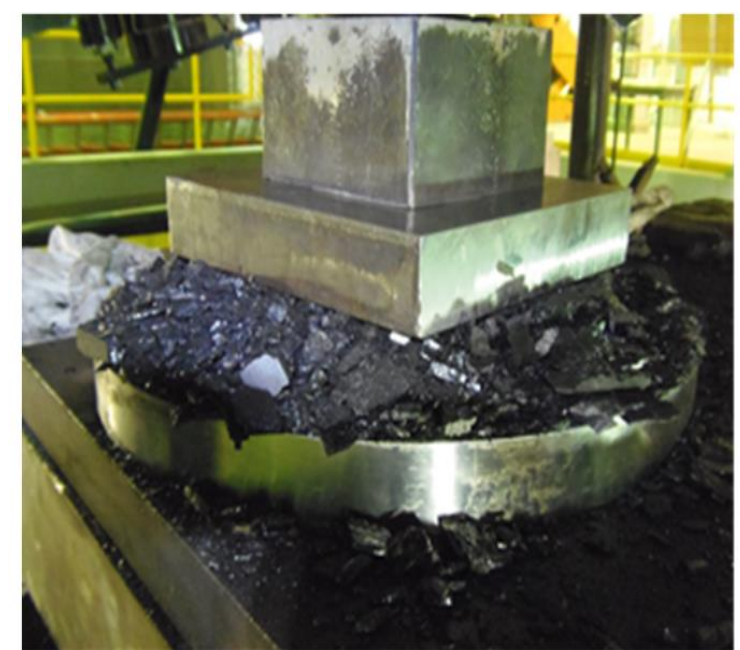

Sample \# 15 after testing, reloading

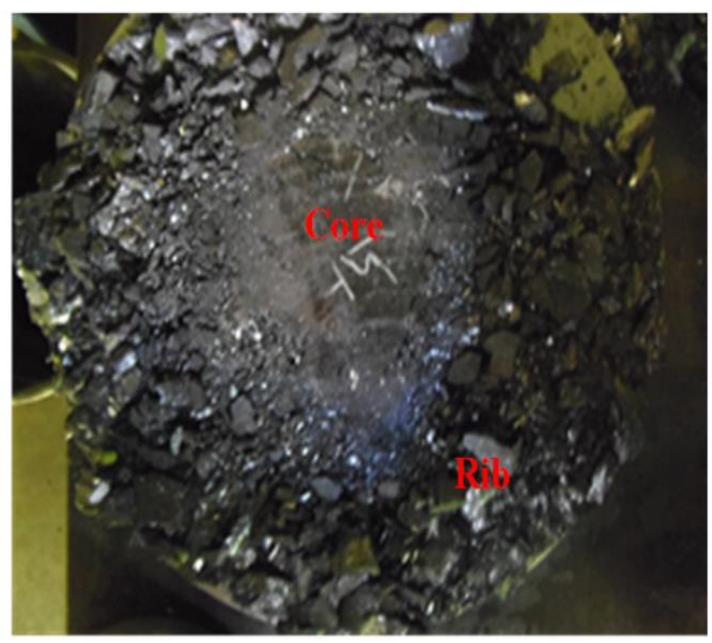

Sample \# 15 after testing, reloading

Figure 5-13 Reloading of coal specimen No. 15 until its failure 
Figure 5-15 shows the loading-reloading stress-strain curves for coal specimen \#15. It is obvious that its ultimate strength in the first stage of loading was higher than that of the reloading stage, because it was expected that some micro-cracks would initiate and grow during the first loading stage.

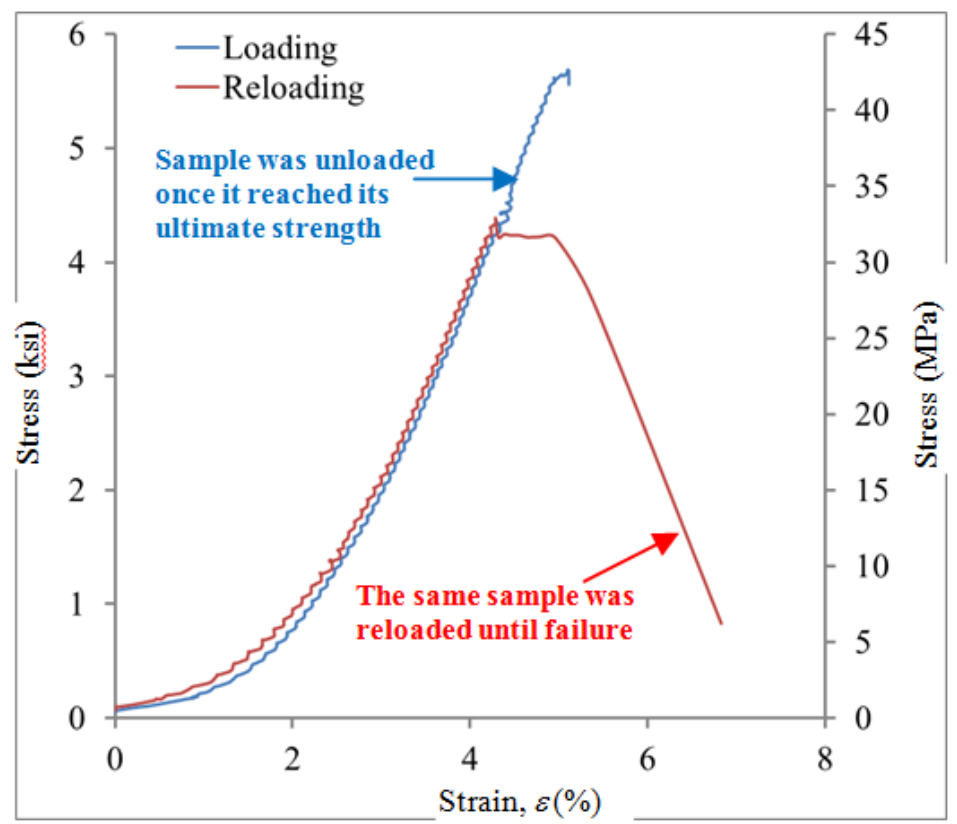

Figure 5-14 Loading-reloading stress-strain curves of coal specimen No. 15 in group 4

Fracture initiation is manifested by departure from the linearity of the stress-strain curve near ultimate strength. These cracks, however, did not propagate sufficiently to cause complete failure of the specimen because the load was released once ultimate strength was reached. Moreover, the specimen ribs were slightly deteriorated after the first loading as shown in Figure 5-12. Hence, the confinement from the edges and ribs in the reloading stage would be smaller than the first stage of loading. Therefore, the ultimate strength of the specimen in the reloading stage was expected to be smaller than the loading stage. 
In summary, all coal specimens in group 4 with interface friction $=0.25$ and $\mathrm{W} / \mathrm{H}=$ 1-3.6 failed suddenly and violently except two specimens whose failure was sudden, but not violent in terms of noise and debris ejection. These two coal specimens had exceptionally low vertical stress results.

\subsubsection{Group 5}

For group 5, the $\mathrm{W} / \mathrm{H}$ ratio of coal specimens ranged from 4.8 to 8.5 and the interface friction was 0.25 . The test results from this group showed that when the $\mathrm{W} / \mathrm{H}$ ratio of specimen was between 4.8 and 7.5 the failure is violent, and it was observed that highstrength coal specimens failed more violently than low-strength ones. However, when the $\mathrm{W} / \mathrm{H}$ ratio is equal to or greater than 7.7 the mode of failure is smooth and non-violent with a strain hardening mode.

Figure 5-16 shows an example of the results of unstable sudden and violent failure for a coal specimen in group 5. Specimen \#33 had a cross-sectional dimension of $132.1 \times 134.6 \mathrm{~mm}(5.2 \times 5.3 \mathrm{in})$ and a height of $25.4 \mathrm{~mm}(1.0 \mathrm{in})$ with a $\mathrm{W} / \mathrm{H}=5.3$. The rib zone was damaged completely and separated from the core zone of 101.6 x $101.6 \mathrm{~mm}$ (4 $\mathrm{x} 4 \mathrm{in}$ ). On the other hand, coal specimens with a $\mathrm{W} / \mathrm{H}=7.7$ or larger did not fail violently. Its load was released once it reached $2891 \mathrm{KN}$ (650 kips) which is equivalent to a state of stress of more than $118.6 \mathrm{MPa}(17.2 \mathrm{ksi})$. 


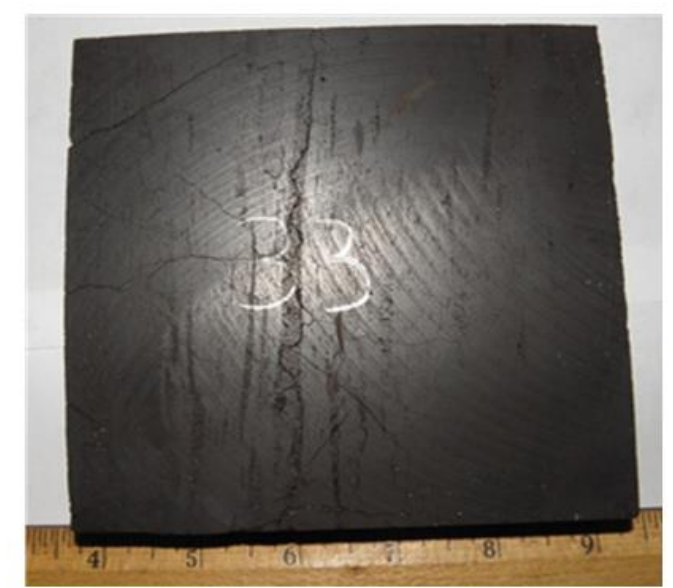

Sample \# 33 before testing

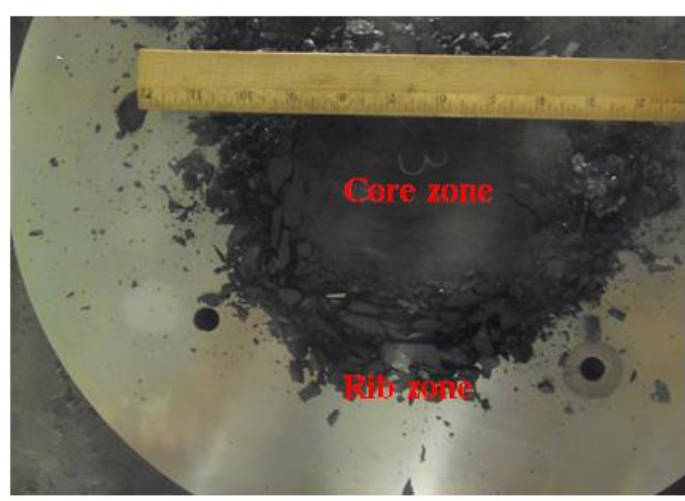

Size of the core zone

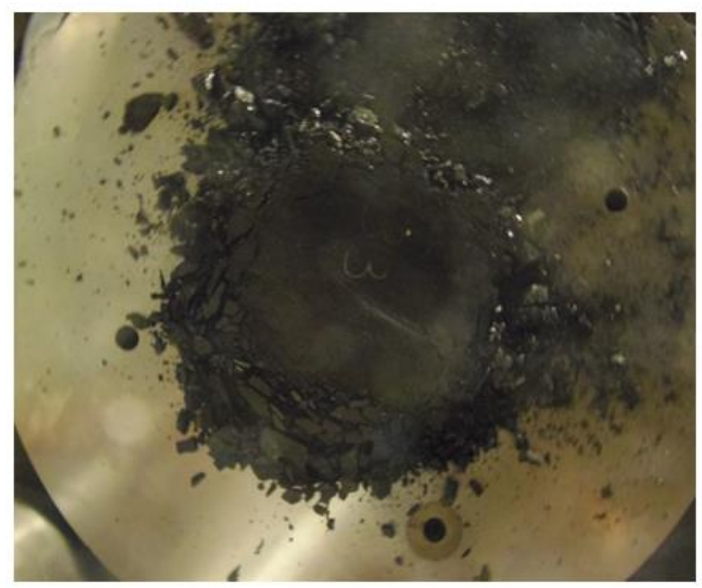

Sample \# 33 after testing

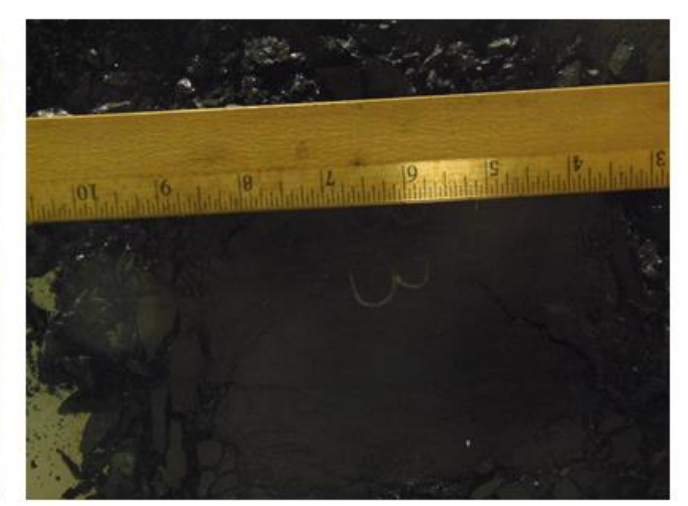

Figure 5-15 Specimen No. 33 in group 5 and its rib and core zones after testing

Two zones can be recognized on the top surface of the failed coal specimen, rib zone and core zone. In general, the rib zone is damaged completely because the coal particles in the rib zone are disconnected and separated from each other. The core zone is highly damaged; it would break if it was not handled with extreme care after testing. It was also found that the size of core zone increases with increasing the $\mathrm{W} / \mathrm{H}$ ratio. Rashed and Peng (2013) found that the core zone is the main cause for violent failure of coal specimens. 
Figure 5-17a \& 5-17b show representative examples of stress strain curves for coal specimens in group 5, the failures of which were stable/non-violent and unstable/violent, respectively. As shown in Figure 5-17a, there was a clear reduction in stiffness after point A, indicating that the coal specimens had been damaged. This was substantiated by the specimens being easily splitting apart during handling and transporting after testing. The strain energy stored in the specimens was released in the deterioration process during the strain hardening stage. After the load was released once it reached 2,891 KN (650 kips) or a stress of $118.6 \mathrm{MPa}(17.2 \mathrm{ksi})$, the dissipated energy was much more than the elastic energy stored in the coal specimens. Hence, the burst index would be smaller than one, thereby very unlikely to bump.

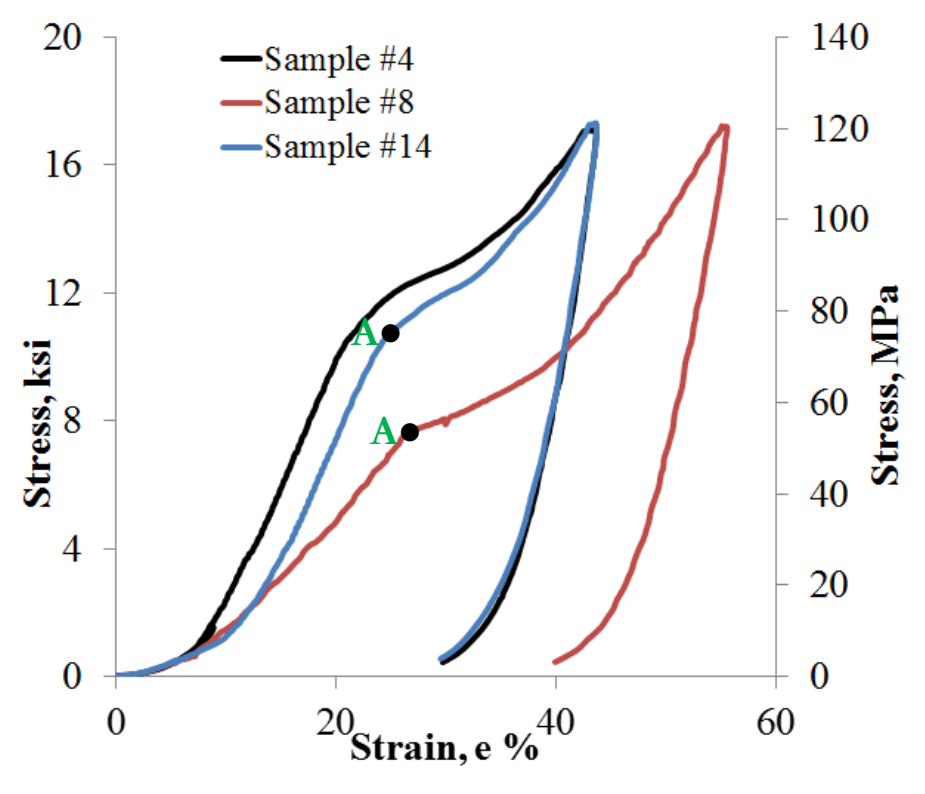

a. Non violent failure 


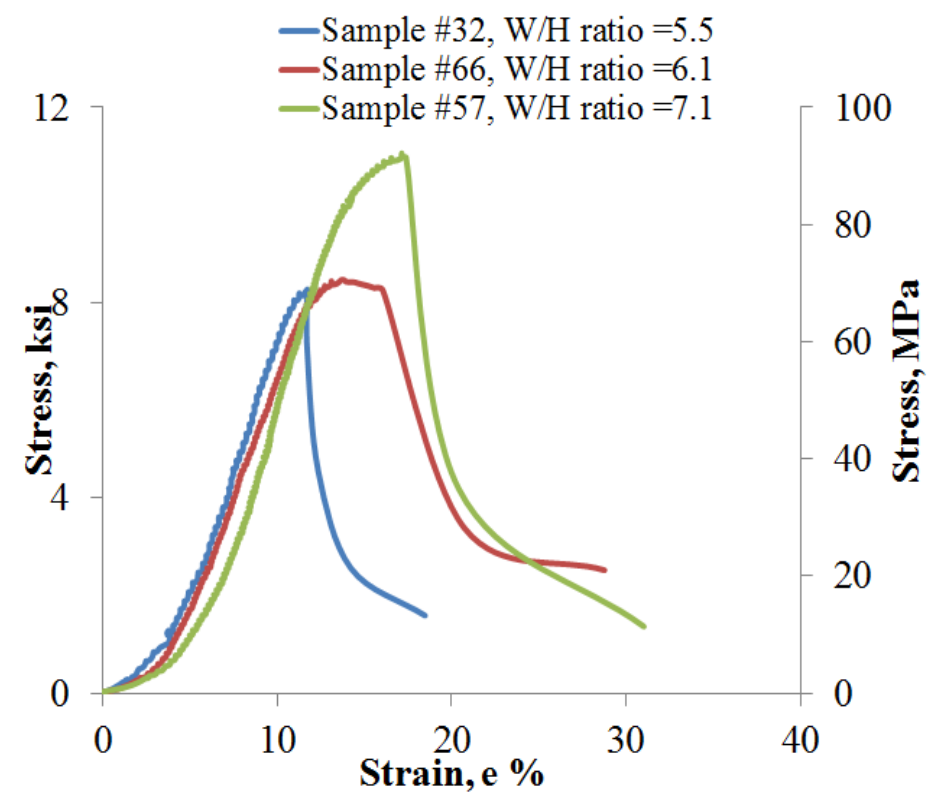

b. violent failure

Figure 5-16 Stress-strain curves for a) non-violent failures and b) violent failures for coal specimens in group 5

Moreover, as shown in Figure 5-17a at 118.6 MPa (17.2 ksi), specimens \#14, \#4 and $\# 8$ were subjected to strains of $0.4,0.4$, and $0.52 \mathrm{in} / \mathrm{in}$, or 40,40 and $52 \%$, respectively. The high \% strain indicated that due to the high compressive load the coal specimen was crushed and pulverized. Further increase in the load on the specimens would not change their mode of failure, but would be more pulverized. Please note that the strain at maximum load shown in Figure 5-17a for the three coal specimens that did not fail violently was more than 10 times that shown in Figure 15 for a coal specimen that failed violently.

Figure 5-17b shows the typical stress strain curves for specimens whose failure was sudden and violent when the $\mathrm{W} / \mathrm{H}$ ratio ranges from 4.8 to 7.5 . The three coal specimens in Figure 5-17b, have $\mathrm{W} / \mathrm{H}$ ratios $5.5 \& 6.1$ and 7.1 , failed instantaneously with sudden 
reduction in strength once the ultimate strength was reached. The failure of these coal specimens was very violent in terms of noise and ejections.

Therefore, the test results showed that under static loading condition, when the interface friction between the loading platens and the coal specimen is equal to 0.25 and the $\mathrm{W} / \mathrm{H}$ ratio of the coal specimen is larger than 7.7, no bump would occur. Consequently, it was found that no static load would cause violent failure of coal specimens with interface friction $=0.25$ or less and $\mathrm{a} \mathrm{W} / \mathrm{H}=7.7$ or more.

If a coal specimen has an interface friction of 0.25 and $\mathrm{W} / \mathrm{H}$ ratio larger than 7.7 fails violently, it would not be due to a static load, but most likely from a dynamic load including an impact load applied in combination with the static load. At higher interface frictions, however, it is expected that the threshold $\mathrm{W} / \mathrm{H}$ ratio at which the mode of failure would change from violent to non-violent would increase from 7.7 to a higher value. Since the coal specimens are a small scale models for the coal pillars. Hence, the general conclusion drawn from the laboratory work conducted in chapter five can be extended to coal pillars of the same conditions. Therefore, by analogy, the shock bump proposed by Rice is more likely to be the main cause for a bump when a pillar of $\mathrm{W} / \mathrm{H}$ ratio $=7.7$ or larger and interface friction of 0.25 failed violently. Many documented cases support this idea, where bump-prone coal seams are always overlain by a strong and stiff stratum or strata such as limestone, sandstone, or massive shale (Peng, 2008).

\subsection{Investigating the Failed Core Zone of Coal Specimen}

The main objective of this section is to prove by experimental testing that the core zone of the failed coal specimen is neither elastic nor intact. However it is damaged to some extent. Thirteen (13) coal specimens of $\mathrm{W} / \mathrm{H}$ ratio ranges from 3.7 to 5.1 were tested 
using the WVU MTS machine under a constant load control of $315 \mathrm{lbf} / \mathrm{sec}$, where the interface friction between the coal specimens and the machine platens is 0.25 and 0.1 .

To examine whether the core zone of the failed coal specimen is elastic or not, the following methodology was adopted: 1) Coal specimens have been tested under a load control and once they failed, the load is released and then the rib zone is cleared away because it is completely damaged, while the core zone is reloaded again. 2) The forcedisplacement curve for the core zone was compared with that of the intact coal specimen. 3) It is believed that the core zone of the failed coal specimen would be elastic when the force-displacement curve for the core zone of failed coal specimen is similar to that of an intact coal specimen, i.e., it has to exhibit a well-defined linear relationship between the force and the displacement or elastic deformation. Followed by non-linear relationship between the force and the displacement, plastic deformation, and then strain softening. Second, the stiffness ratio of the intact coal specimen and core zone of failed coal specimen has to be close.

Figure 5-18 shows a coal specimen \# 10 before and after failure, it had a crosssectional dimension of $3.9 \times 4.1$ in and a height of 0.82 in, making the $\mathrm{W} / \mathrm{H}$ ratio is 4.9 . The interface friction between the coal specimen and the loading platens is 0.25 . The coal specimen failed so violent with very high ejections and loud pitch noise. Note how much ejected debris is on and under the lower platen, it is shown in Figure 5-18.c. Two zones can be recognized on the top surface of the failed coal specimen, rib and core zones, as shown in Figure 5-18.d. The core zone has an irregular shape. Calculating accurate stress is difficult to accomplish since it requires precise determination of the cross-sectional area. 
Therefore, it is preferable to present and study the force-displacement curve rather than the stress-strain curve of the core zone.

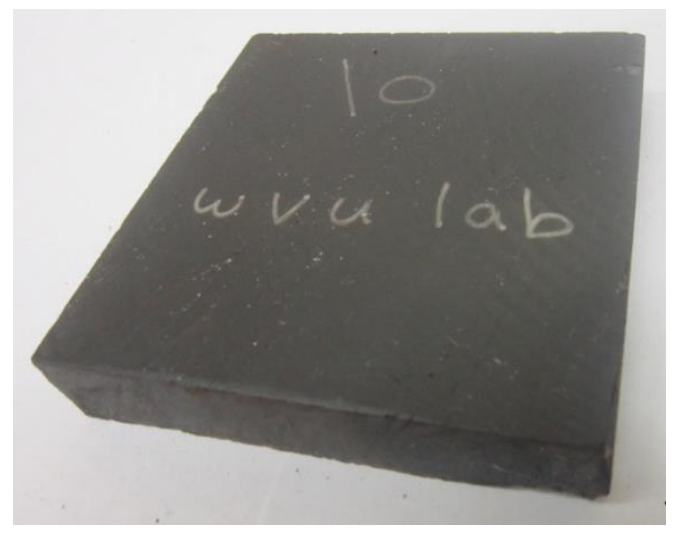

a. Specimen \# 10 before testing

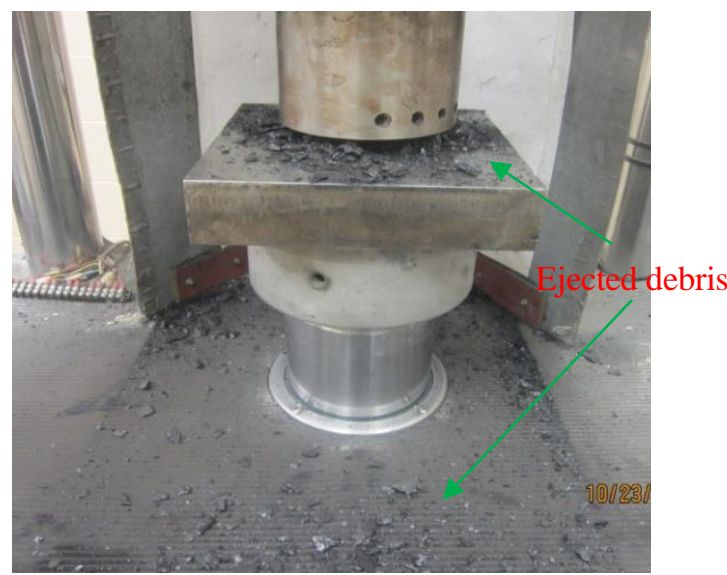

c. Ejected debris after failure

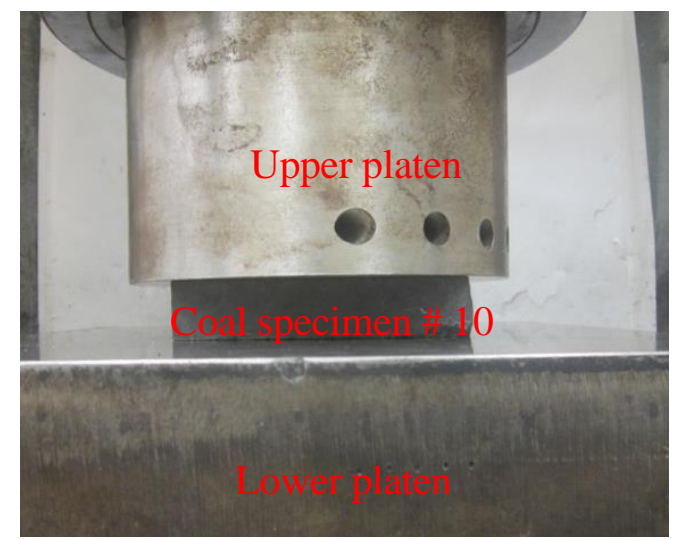

b. Specimen \# 10 before testing

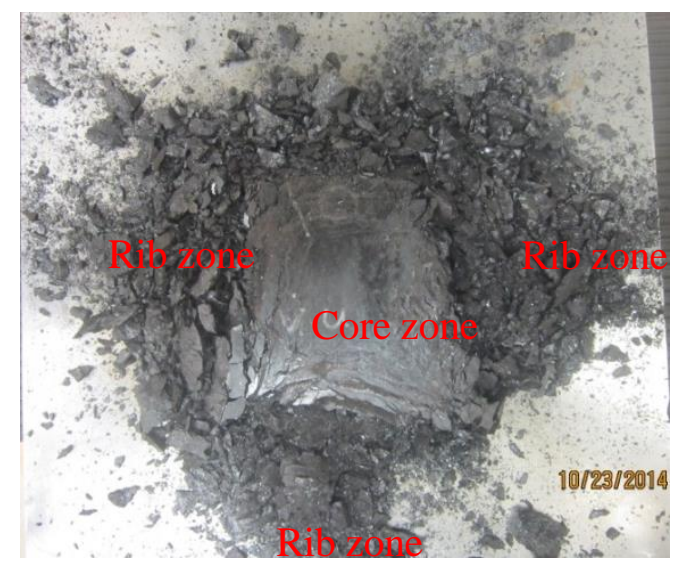

d. Core and rib zones for failed coal

Figure 5-17 Coal specimen No. 10 before and after failure

Figure 5-19 shows the force-displacement curve for a coal specimen \# 10. This curve can be divided into 4 stages: During the first stage, the pre-existing micro-cracks are closed which causes initial no-linearity between the force and displacement. During stage 2, the coal specimen has linearly elastic behavior. During stage 3 , the coal specimen is undergone a rapid acceleration of micro-cracking and volume increase, plastic deformation. During stage 4, the coal specimen passed the ultimate strength, the specimen is undergone 
strain softening behavior (failure). It is believed that the core zone of failed coal specimen would be elastic if and only if it follows these four stages upon loading.

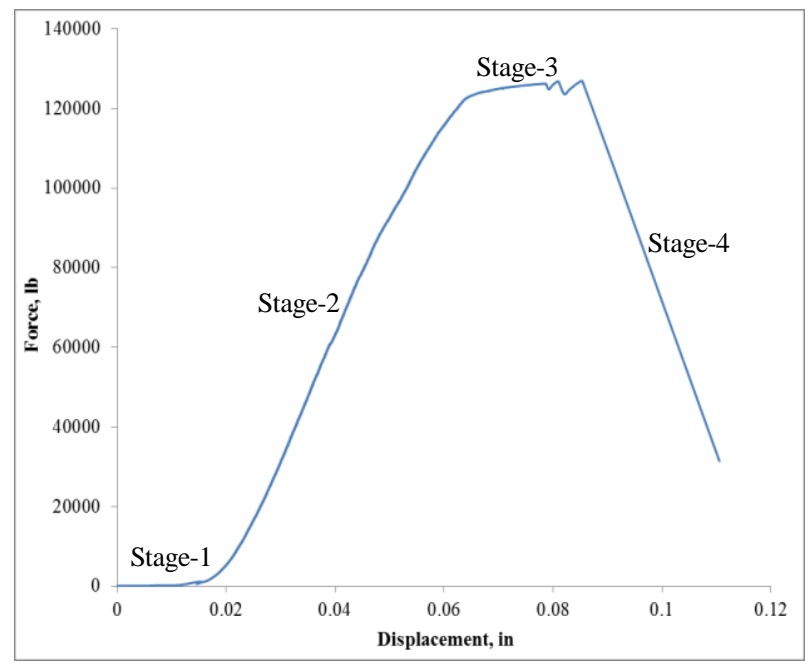

Figure 5-18 Force-displacement curve for intact coal specimen No. 10

Once the coal specimen \# 10 failed, the rib zone which is completely fractured and separated from the core zone is disposed off as shown in Figure 5-20.a \& b and then only the core zone of the failed coal specimen is reloaded as shown in Figure 5-20.c. Figure 520.d demonstrates the core zone after reloading where the displacement reaches 0.35 in. Note that the original height of the coal specimen was 0.82 in. Hence the specimen was experiencing $0.42 \mathrm{in} / \mathrm{in}$ strain which is very high.

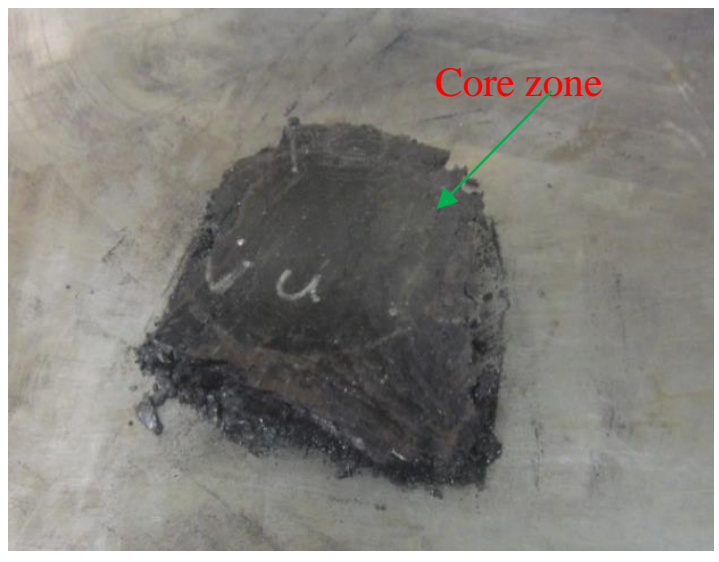

a. the core zone of a failed specimen before reloading

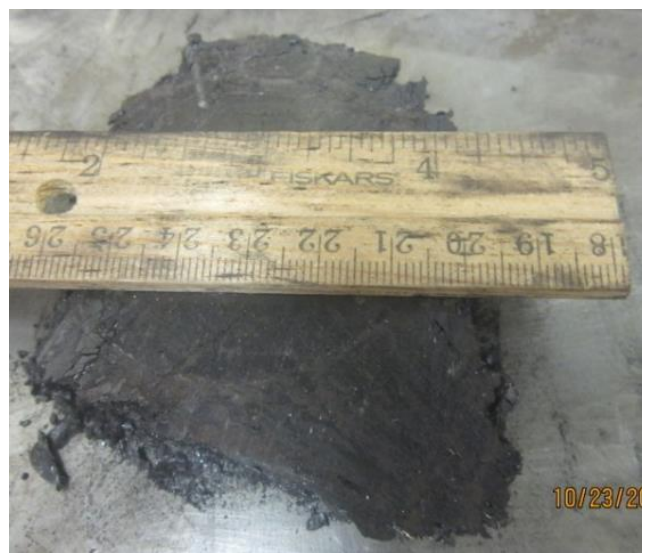

b. the size of a core zone of a failed coal specimen 


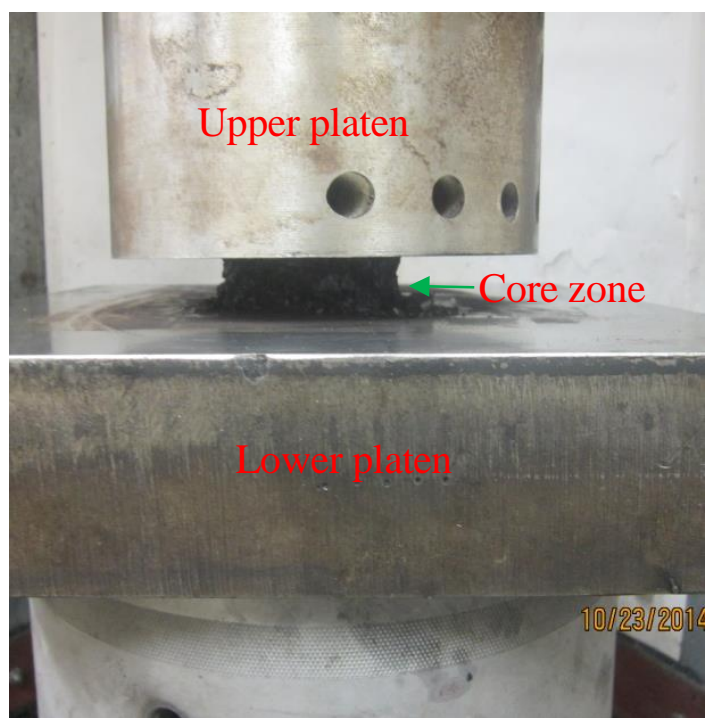

c. The core zone of a failed specimen before reloading

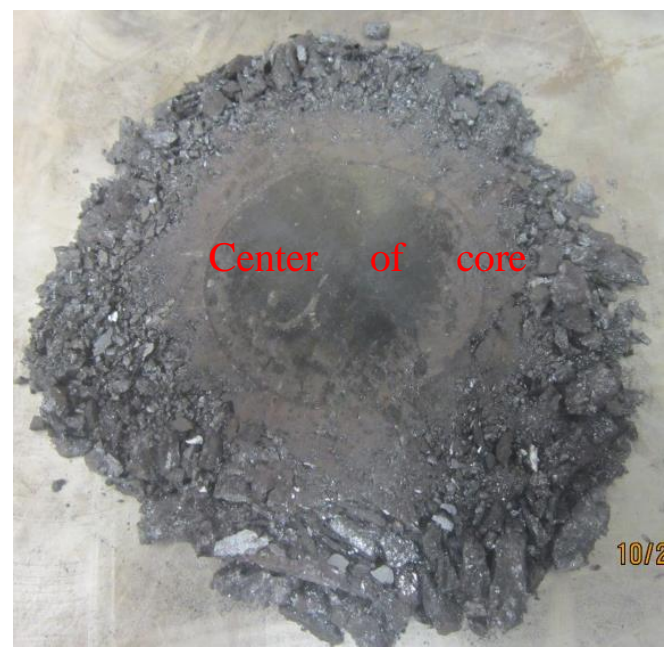

d. the core zone after reloading

Figure 5-19 Core zone of specimen No. 10 before and after reloading

Figure 5-21 shows the force-displacement curves for the intact coal specimen \# 10 (blue line) and reloading core zone for coal specimen \# 10 (red line). Note the difference in force-displacement curves for intact coal specimen (blue line) and core zone of failed coal specimen (red line). The force-displacement curve for core zone can be divided into three stages from 0 to A (concave upward), from A to B (straight line) and from B to C (concave upward) where the load was stopped. It is believed that the core zone is not elastic because first, the force-displacement curve for core zone is completely different from that of intact coal specimen (see Figure 5-21). Second, since the force-displacement concave upward from $\mathrm{B}$ to $\mathrm{C}$, which basically indicates compaction of the core zone, that means the core zone is not elastic and it is damaged to some extent.

The stiffness of the coal specimen can be calculated from the slope of the straight part of the force vs. the displacement curve. The stiffness of the intact coal specimen \#10 is approximately 2.9 million $\mathrm{lb} / \mathrm{in}$. For the core zone of the failed coal specimen, since the 
stiffness changes with changing the displacement, it has been calculated at two locations: 1) from 0 to $\mathrm{A}$, an initial stiffness is approximately $63,411 \mathrm{lb} / \mathrm{in} .2$ ) from A to B the stiffness is approximately $3961 \mathrm{lb} / \mathrm{in}$. The stiffness of intact coal specimen is approximately 45 times the initial stiffness, and 732 times the secondary stiffness of the damaged core zone specimen. The stiffness of the core zone increases from B to C, because the center of the core zone becomes more compacted.

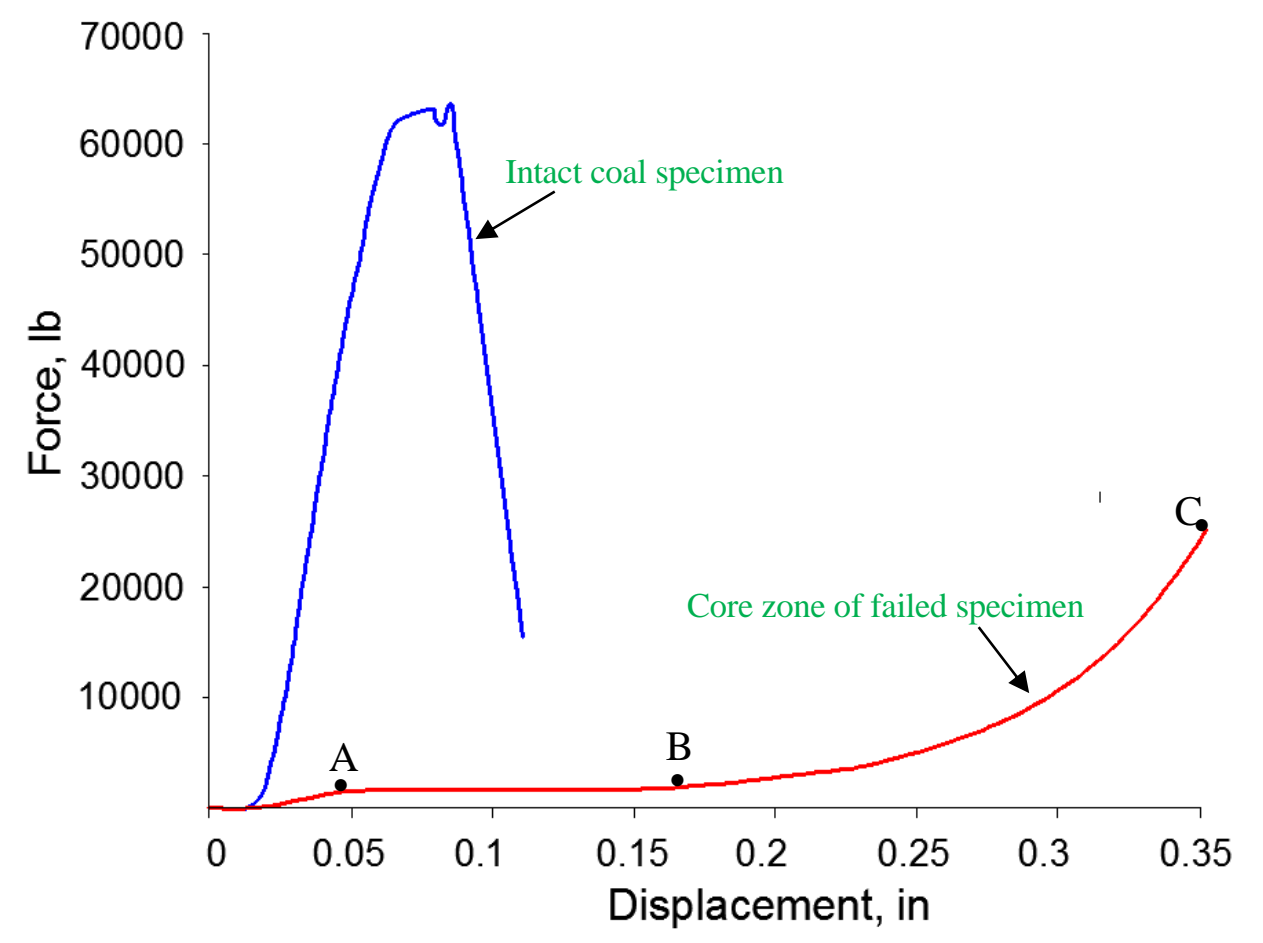

Figure 5-20 Force-displacement curves for core zone and intact coal specimen No. 10

Therefore, this big difference in the stiffness between the intact coal specimen and the core zone of the failed coal specimen, in addition to the non-linearity of forcedisplacement curve for the core zone of the failed coal specimen prove that the core zone is not elastic. Thus, when an intact coal specimen fails under compression, two zones are recognized on the top surface of the failed coal specimen, the rib and the core zones, both 
of them are neither elastic nor intact, i.e., the rib and the core zones have failed when coal specimen fails. However the rib zone is highly damaged comparing to the core zone.

\subsection{Summary for Chapter Five}

The violent failure of coal specimens depends on both the interface friction and $\mathrm{W} / \mathrm{H}$ ratio. With a proper combination of the $\mathrm{W} / \mathrm{H}$ ratio and the interface friction, the mode of failure can be controlled and converted from violent to non-violent. According to the test results, every interface friction is associated with a specific threshold $\mathrm{W} / \mathrm{H}$ ratio above which the mode of failure is stable and non-violent, and below which the failure would be either sudden violent or sudden non-violent. The main conclusions from chapter five can be summarized as follows:

- When the interface friction between coal specimens and the loading platens is 0.1 and the $\mathrm{W} / \mathrm{H}$ ratio ranges from 1 to 3.8 , the expected mode of failure is sudden, but not violent in terms of noise and ejection. So even a very low interface friction does not prevent the sudden failure as shown in group 1.

- When the interface friction is 0.1 and the $\mathrm{W} / \mathrm{H}$ ratio ranges from 4.7 to 6.5 , the mode of failure changes from brittle to ductile failure with clear plastic strain and permanent deformation. However the mode of failure is strain softening as shown in group 2.

- When the interface friction is 0.1 and the $\mathrm{W} / \mathrm{H}$ ratio ranges from 6.8 to 10 , failure is very stable with strain hardening. Coal specimens expand and are squeezed with very low noise and ejection. This combination of interface 
friction and $\mathrm{W} / \mathrm{H}$ ratio causes stable slip along the interface and eventually smooth failure as shown in group 3.

- When the interface friction is 0.25 and the $\mathrm{W} / \mathrm{H}$ ratio is less than 7.7, failure is sudden and violent, and the intensity of violence increases with increasing the vertical stress. $\mathrm{W} / \mathrm{H}$ ratio $=7.7$ is the threshold limit between violent and non-violent failures. So, the coal specimens experience Stable slip along the interface when the $\mathrm{W} / \mathrm{H}$ ratio $=7.7$ and the interface friction $=0.25$.

- Therefore, based on the test results in chapter five, bumps would occur only when the interface friction is 0.25 and the $\mathrm{W} / \mathrm{H}$ ratio is less than 7.7. Other combination of $\mathrm{W} / \mathrm{H}$ ratio and interface friction higher than 0.25 remains to be determined.

- When coal specimen fails, two zoned are recognized on the top surface of failed coal specimens, the rib and core zones. The rib zone includes edges and corners, while the core zone is the central portion of the coal specimen. It was found that the rib zone is highly (almost completely) damaged compared to the core zone, which is also damaged to some extent, however less than the rib zone. Once the core specimen fails, the core zone is no longer elastic. 


\section{CHAPTER 6: MITIGATING THE VIOLENT FAILURE OF COAL SPECIMENS}

\subsection{Introduction}

When coal mine bumps occur, coal fragments from the pillars are blasted into the mine entries. Mine equipment may be damaged or destroyed. Mine personnel may be injured or even killed. Therefore, reducing or eliminating coal mine bumps is necessary in today's highly competitive coal industry that requires safe workplaces.

In chapter six, an attempt has been conducted to mitigate the violent failure of coal specimens subjected to uniaxial compression test. It has been demonstrated by laboratory test results shown in Chapter three that there is a positive correlation between the UCS (or the vertical strength when $\mathrm{W} / \mathrm{H}$ ratio is higher than 0.5 ) of coal specimens and their violent failure. Three hypotheses have been assumed to eliminate or reduce the violent failure of coal specimens. The first hypothesis is that violent failure is restricted to rib zone only. The idea here is that if the ribs of a coal specimen are soft, they are expected to fail gradually and gently, and the load on them is transferred to the deeper core until the whole coal specimen fails. Conversely if the ribs of a coal specimen are so stiff that they do not fail gradually, but keep storing more energy until the moment of failure, these ribs have the least confinement so they fail with so much suddenly-released energy from them that might cause violent failures. The second hypothesis is that violent failure is restricted to the core zone (the central portion of coal specimen). The core does not yield because of high confinement, and hence it can store so much energy that could be released suddenly and violently at the moment of failure. The third hypothesis is that sudden violent bump occurs 
both at ribs and core zones. So to test these hypotheses, coal specimens were softened either partially or completely. A coal specimen can be softened partially by weakening only the ribs or the central portion of coal specimen through drilling holes at specified locations. On the other hand, a coal specimen can be softened completely by immersing it in water for 24 hours and then to be tested directly.

In order to quantify coal mine bumps in the laboratory, several parameters have been determined. Some of these parameters are measured quantitatively such as the unconfined compressive strength (or the vertical strength when the W/H ratio is higher than $0.5)$, the modulus of elasticity and whether there is a sudden loss of strength or not. Other parameters were measured qualitatively, such as the degree of ejection and the magnitude of noise. The qualitative scale employed ranged from low to very high, depending on the author's experience during the testing of the coal specimens.

The failure of a coal specimen is violent, when there is a sudden loss of strength accompanied by high ejection of coal and loud noise. On the other hand, the failure is nonviolent when it is accompanied by little ejection of coal, low noise and no sudden loss of strength. All coal specimens tested in this chapter are from the Sunnyside seam, Utah coal.

\subsection{Testing the First Hypothesis to Mitigate the Violent Failure}

To test the first hypothesis, the rib zone of the coal specimen was softened by drilling horizontal holes (i.e., perpendicular to the rib surface) $1 / 10$-in in diameter by $1 / 2$-in deep. These artificial holes drilled in the ribs of the coal specimen would facilitate initiation of new cracks during the loading process and hence might release the stored energy gradually. The distance between these holes drilled in the rib zone is $1.0 \mathrm{in}$. 
Figure 6-1 shows the locations of these holes used to soften the ribs for a coal specimen of $\mathrm{W} / \mathrm{H}$ ratio $=3$. The idea behind softening the ribs of the coal specimen is that because coal is a brittle material, so a sudden failure of these ribs could trigger excessive and sudden load transfer to the remaining intact parts of the coal specimen. Therefore, a violent failure could be avoided when these ribs fail smoothly and gently due to the existence of these drilled holes.

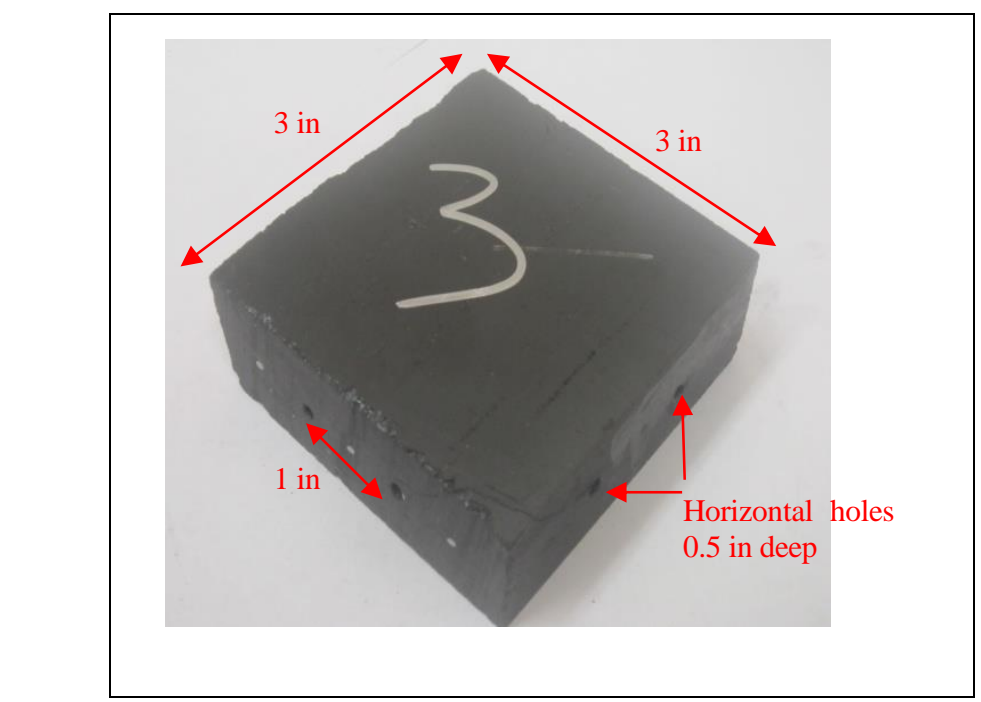

Figure 6-1 Location of the drilled horizontal holes for a coal specimen of W/H ratio $=3$

Coal specimens of square cross-sectional area and width-to-height ratio $=3.0$ and 4.0 were tested under constant strain control where the rate of displacement was $10^{-4} \mathrm{in} / \mathrm{sec}$. The height of the specimens was kept constant and equal to 1 in. Coal specimens of W/H ratio greater than 4 could not be tested because of the limit load capacity of the WVU MTS machine.

Table 6-1 shows the test results for coal specimens of $\mathrm{W} / \mathrm{H}$ ratio $=3$ and 4 , subjected to vertical stress. Note that the term "vertical stress" is used rather than the UCS, because the width-to-height ratios of the coal specimens are higher than 0.5 . The ribs of 
these coal specimens were softened by horizontal holes. For coal specimens of $\mathrm{W} / \mathrm{H}$ ratio $=$ 3, only specimen \#3 failed violently among seven specimens and lost $85 \%$ of its strength suddenly once it reached its peak strength. The amount of ejection and the degree of noise were very high during failure of the specimen. Specimen \#3 attained the highest vertical stress and Modulus of elasticity among all coal specimens of $\mathrm{W} / \mathrm{H}$ ratio $=3$. Hence, the higher the vertical stress of the coal specimen, the higher the strain energy and the hence the higher the potential for violent failure. Coal specimens \#1 and \#5 have the lowest ejections and noise during failure. When the strength of coal specimens is higher than 6,439 psi, the amount of ejected debris and the degree of noise pitch increase. This might provide an indication about the threshold value of strength that separates the violent from non-violent failure for Utah coal. So for coal specimen of $\mathrm{WH}$ ratio $=3$, as long as the strength is less than 6,439 for the coal specimens used in this research, the failure will not be violent.

For coal specimens of $\mathrm{W} / \mathrm{H}$ ratio $=4$, all coal specimens failed violently with very high ejection and noise except specimen \#6. Specimen \#6 had the lowest vertical strength among coal specimens of $\mathrm{W} / \mathrm{H}$ ratio $=4$. Although specimen $\# 6$ did not fail violently, its failure was not smooth. Rather it was accompanied by moderate ejection and high noise. This indicates that for coal specimen of $\mathrm{W} / \mathrm{H}$ ratio $=4$, the strength should be less than 8,330 psi to fail non-violently. 
Table 6-1 Test results for coal specimens where the ribs were softened by horizontal holes

\begin{tabular}{|c|c|c|c|c|c|c|c|c|c|c|c|}
\hline $\begin{array}{c}\text { Specimen } \\
\#\end{array}$ & $\begin{array}{l}\text { W/H } \\
\text { ratio }\end{array}$ & $\begin{array}{c}\text { Vertical } \\
\text { strength, } \\
\text { psi }\end{array}$ & $\begin{array}{c}\text { Vertical } \\
\text { strength }_{\text {avg }}, \\
\text { psi }\end{array}$ & $\begin{array}{c}\text { Vertical } \\
\text { strength- } \\
\text { std., psi }\end{array}$ & E, psi & $\mathbf{E}_{\text {avg }}$, psi & $\begin{array}{c}\text { E-std., } \\
\text { psi }\end{array}$ & $\begin{array}{c}\text { Failure } \\
\text { type }\end{array}$ & Ejection & Noise & $\begin{array}{c}\text { Sudden loss of } \\
\text { strength }\end{array}$ \\
\hline 1 & \multirow{6}{*}{3} & 6,331 & \multirow{6}{*}{6,864} & \multirow{6}{*}{551} & 298,630 & \multirow{6}{*}{301,167} & \multirow{6}{*}{19,650} & Non-violent & Low & Low & No \\
\hline 2 & & 6,707 & & & 323,610 & & & Non-violent & Moderate & Moderate & No \\
\hline 3 & & 7,873 & & & 318,990 & & & Violent & V. high & V. high & Yes, $85 \%$ of UCS \\
\hline 5 & & 6,439 & & & 268,125 & & & Non-violent & Low & Low & No \\
\hline 6 & & 6,862 & & & 298,590 & & & Non-violent & High & High & No \\
\hline 7 & & 6,974 & & & 299,056 & & & Non-violent & Moderate & low & No \\
\hline 1 & \multirow{5}{*}{4} & 8,880 & \multirow{5}{*}{8,800} & \multirow{5}{*}{267} & 235,104 & \multirow{5}{*}{240,377} & \multirow{5}{*}{14,185} & Violent & V. high & V. high & Yes, $92 \%$ of UCS \\
\hline 2 & & 9,000 & & & 264,472 & & & Violent & V. high & V. high & Yes, $93 \%$ of UCS \\
\hline 3 & & 8,886 & & & 228,787 & & & Violent & High & V. high & $\begin{array}{c}\text { Yes on } 3 \\
\text { consecutive stages } \\
\end{array}$ \\
\hline 5 & & 8,905 & & & 232,515 & & & Violent & V. high & V. high & Yes, $78 \%$ of UCS \\
\hline 6 & & 8,330 & & & 241,009 & & & Non-violent & Moderate & High & No \\
\hline
\end{tabular}


Therefore, softening the ribs is effective in mitigating the violent failure for coal specimens of $\mathrm{W} / \mathrm{H}$ ratio $=3$. Conversely for width-to-height ratio equals to 4 , softening the ribs is not effective in mitigating the violent failure. Note that the depth of the drilled holes is the same for coal specimens of width-to-height ratios 3 and 4 . Hence, a 0.5 in hole depth is not enough to soften a coal specimen of width-to-height ratio 4. It is very likely that the failure of the ribs does not cause the violent failure and the failure of the core might be the main reason for the violent failure, and to mitigate the violent failure, the core zone must be penetrated to some extent. For coal specimens of width-to-height ratio equals to 3 , a 0.5 in deep hole is enough to penetrate the core zone and hence mitigate the violent failure.

Brauner (1994) used a method called drilling yield technique to check the highly stressed zones in the longwall face, where a hole 2-3 in. diameter was drilled in the coal seam ahead of a working face (Peng, 2008). Observations in Polish coal mines indicated that a safe mining state exists when the high stress zone is located a distance greater than 3.4 H, where $\mathrm{H}$ is the mining height (Peng, 2008). A high stress zone is located some distance ahead of the longwall face.

Therefore, to mitigate the violent failure of coal specimen, the high stress zone must be penetrated by these horizontal holes. In order to prove this conclusion, more experiments on coal specimens will need to be performed with holes of more than 0.5 in deep. The author did not succeed to drill holes more than 0.5 in deep, because the coal specimens broke during drilling.

By comparing the test results shown in Tables 3-1, in chapter 3, where coal specimens did not experience any softening and Table 6-1 where the rib zone was softened, it is obvious that the average UCS and Young's Modulus did not experience significant 
reduction by softening the ribs of coal specimens. That was why softening the rib zone is not effective in mitigating the violent failure of coal specimen.

Figures 6-2a and $\mathrm{b}$ shows the stress-strain curves for coal specimens of $\mathrm{W} / \mathrm{H}$ ratio equals to 3 and 4 respectively, where the ribs were softened. Figure 6-2a illustrates that specimen \# 3 has the highest vertical strength compared to all other coal specimens of width-to-height ratio equals to 3 . It was the only specimen that failed violently and there was a sudden loss of strength from almost 6,500 psi to 0 psi. The post-peak slope of this coal specimen was the highest among all other specimens of the same width-to-height ratio that indicated fast crack propagation and hence brittle failure. Conversely, the other specimens with the same $\mathrm{W} / \mathrm{H}$ ratio did not fail violently. Their strength was smaller than that of specimen \#3. 

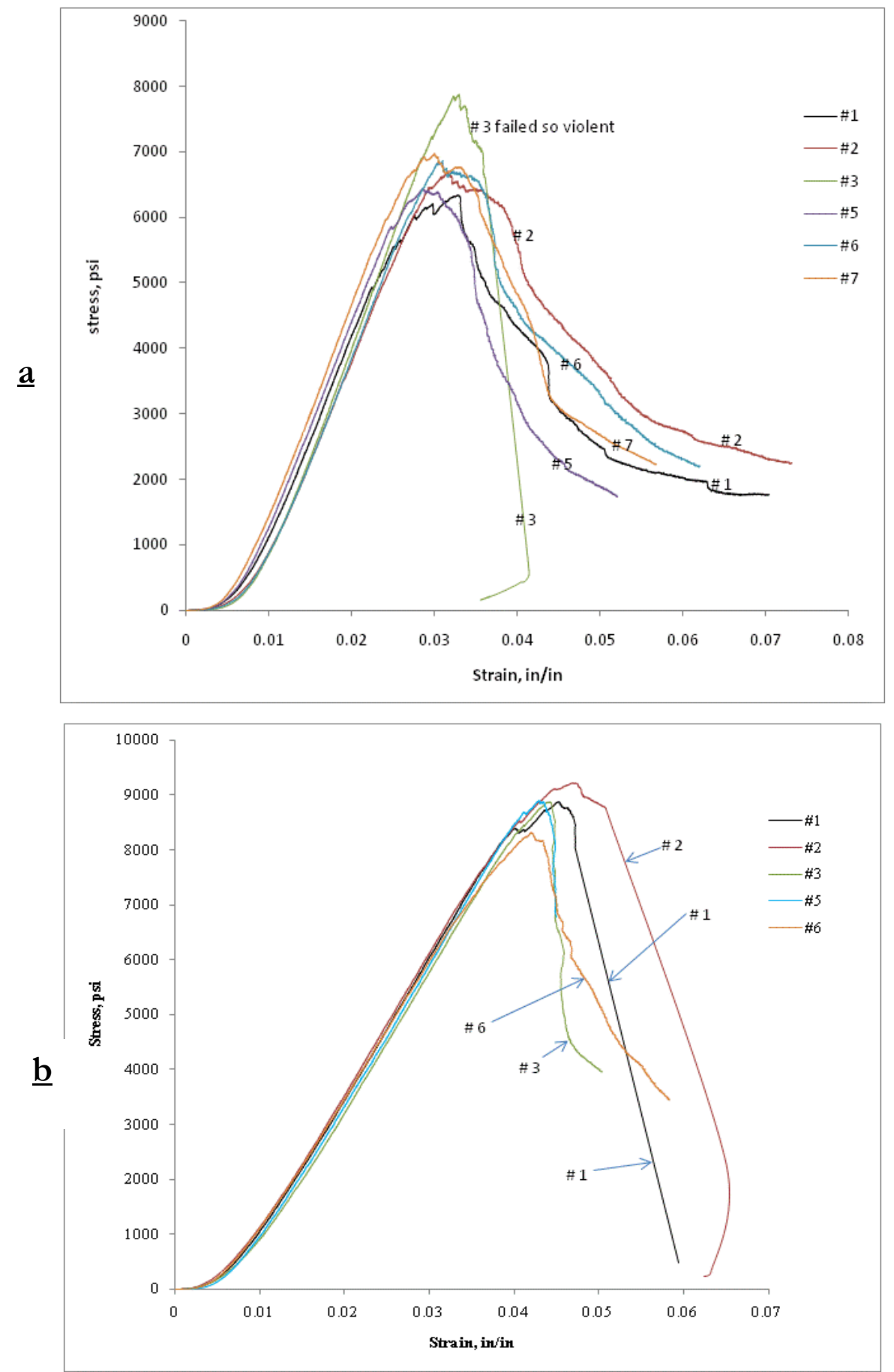

Figure 6-2 Stress strain curves for coal specimens softened by artificial holes: (a) $\mathrm{W} / \mathrm{H}$ ratio $=3$ and (b) $\mathrm{W} / \mathrm{H}$ ratio $=4$ 
Figure 6-2b shows the stress-strain curves for coal specimens of width-to-height ratio equals to 4 . All coal specimens failed violently except specimen \# 6 that had the lowest UCS value. According to the test results shown in Table 6-1 and Figure 6-2b, the higher the UCS, the more potential for violent failure, and the lower the UCS, the less potential for violent failure.

Figure 6-3 shows examples for coal specimens of width-to-height ratio equals to 3 before and after failure. On the top surface of the failed coal specimens (Figure 6-3), two zones can be identified; the rib zone and the core zone. The rib zone has the least confinement and being completely crushed with numerous big cracks. It is believed that the damage variable for the rib zone is 1.0 , which means that the rib is completely crushed. The core zone has the highest confinement and constraint. It is less damaged compared to the rib zone. Hence, the damage index is still less than 1.0. Note that for Specimen \#3, the rib zone was ejected away from the specimen and the failure was very violent. This coal specimen has the highest UCS value among coal specimens of width-to-height ratio equals to 3 . 

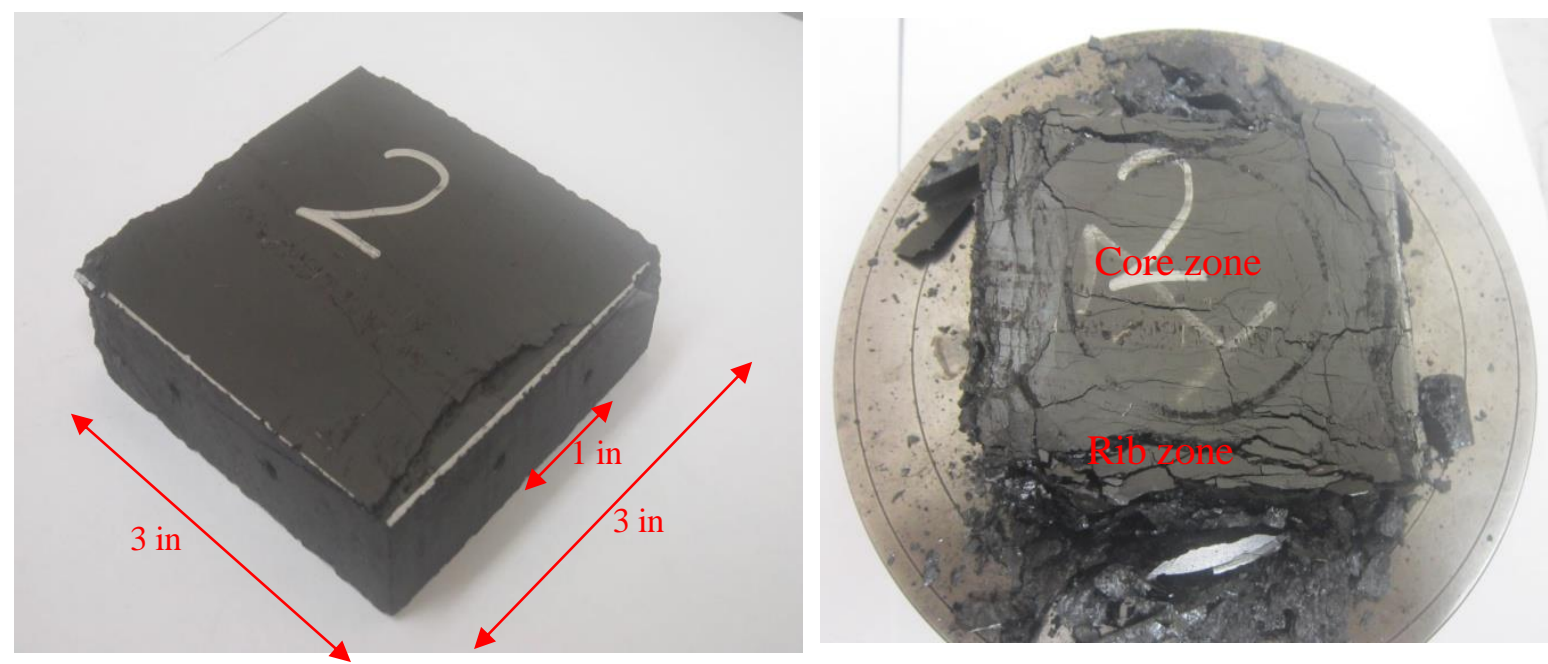

Specimen \# 2
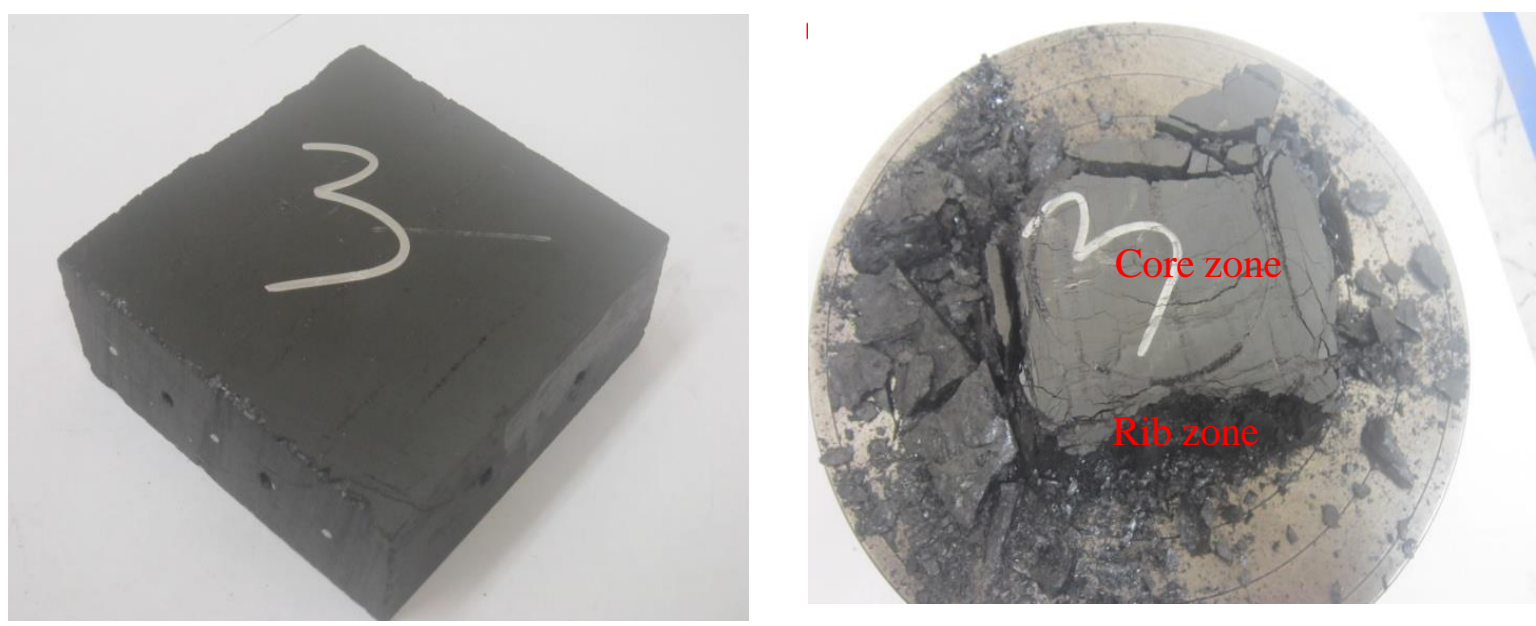

Specimen \# 3

Figure 6-3 Coal specimens of W/H ratio $=3$ softened by artificial holes before and after failure

As shown in Figure 6-3, the rib zone was completely crushed and there are clear visible hair cracks on the top surface of the central portion of the coal specimens, indicating cracks initiation and propagation in the core zone, which gives an indication that the core zone of failed coal specimen is no longer intact once the coal specimen fails. 
The failure pattern of the rib zone is non-uniform. The non-uniformity in the failure pattern could be attributed to the non-uniform distribution of microscopic flaws inside the coal specimen. Stress concentration occurs at these microscopic flaws that amplify the local stresses at their tips. The magnitude of the amplification depends on micro-crack orientations, geometry and dimensions. Moreover, the coal specimens may be to some extent anisotropic. So some parts of the coal specimen might be stiffer than the other parts and can take more loads than the softer parts.

\subsection{Testing the Second Hypothesis to Mitigate the Violent Failure}

Since softening the rib zone did not reduce or eliminate the violent failure of coal specimen of $\mathrm{W} / \mathrm{H}$ ratio $=4$, failure of the rib zone is most likely not the main cause for violent failure. With all mechanical properties being identical, the coal specimen with higher compressive strength should be able to store more elastic strain energy, and hence is more likely to fail violently (Obert and Duvall, 1967). Since the core has the highest confinement, it can store more elastic strain energy which could be transformed into fracture energy and released violently at the moment of failure. This energy could be released violently because of the sudden de-confinement (Gu and Ozbay, 2014). Therefore, softening the core zone through drilling vertical holes will help the coal specimen to expand laterally and convert more of the input energy into inelastic energy. The plastic energy releases slowly, thus nonviolently. The reason was that some of the input energy is dissipated in closing these vertical holes instead of stored as elastic energy in the core zone.

Now a question has been raised, how can the strain energy in the coal specimen be minimized or extracted? Previous experience with modeling coal specimens under uniaxial compression showed that most of the elastic strain energy is stored in the core zone. If the 
energy stored in the core zone is converted into inelastic energy by plastic deformation or any other dissipation process, there will be no violent failure at all. Plastic deformation reduces stress concentrations and causes plastic energy dissipation through creation of new cracks. Therefore, to reduce or eliminate the violent failure of coal specimen, the strain energy stored in the coal specimen must be removed or converted into inelastic energy.

Why elastic strain energy causes violent failure? If the input energy is converted into elastic strain energy, then after the limiting elastic deformation is achieved, the elastic strain energy is completely released instantaneously, thus violently, and causes bumps. It is similar to an elastic spring under compression. Therefore, to avoid sudden release of elastic energy causing bumps, the core of the coal specimen should convert most, if not all, of its input energy into inelastic energy.

Figure 6-4 shows the arrangement of the vertical holes, for specimens of different width-to- height ratios. The diameter of these holes is one-sixteenth of an inch. During the first attempt to soften the core of the coal specimens, when the depth of the holes was equal to the thickness of the coal specimen which was 1 in, the coal specimens were badly fractured. That was why the depth of the holes was reduced from 1 in to 0.8 in to reduce the amount of disturbance to the coal specimens. Please refer to Table 6-2 for the difference in strength for the specimen of $\mathrm{W} / \mathrm{H}$ ratio $=4$ when the depth of the holes is 1.0 and $0.8 \mathrm{in}$. 


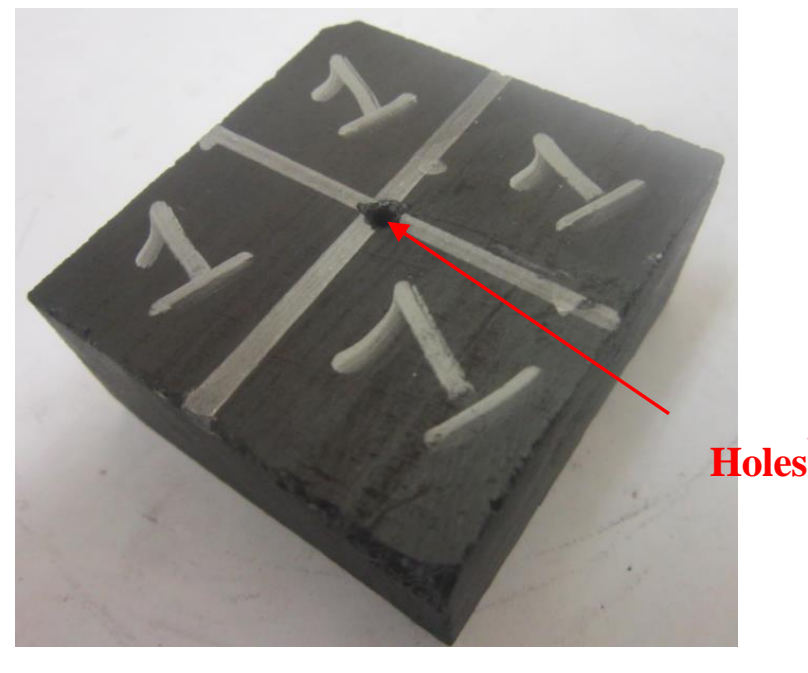

Width-to-height ratio $=2$

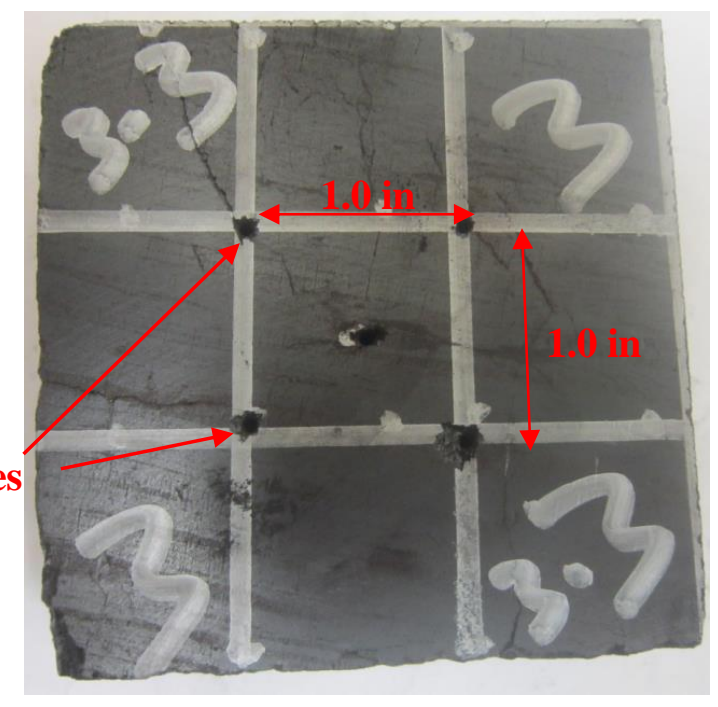

Width-to-height ratio $=3$

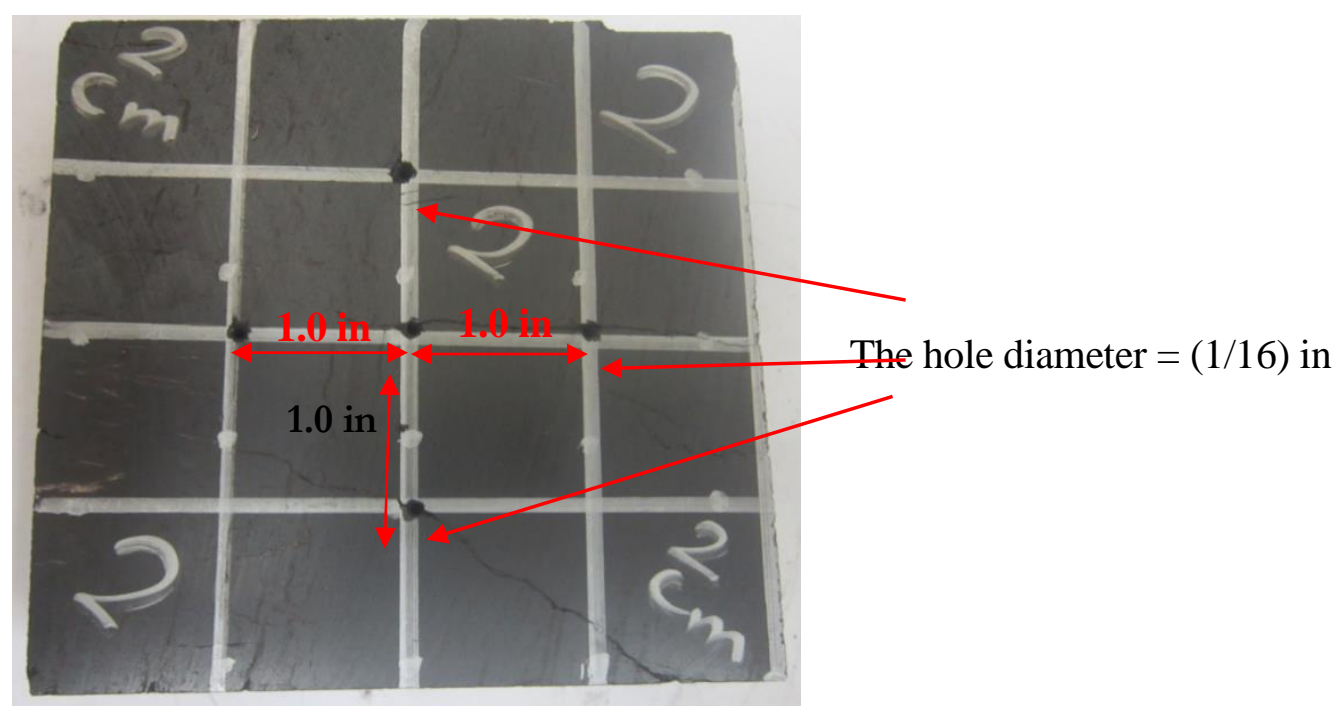

Width-to-height ratio $=4$

Figure 6-4 Arrangement of the vertical holes for coal specimens of different $\mathrm{W} / \mathrm{H}$ ratios

To the author's knowledge, there has been no experimental work performed before about how to soften the core of coal specimen. So there are no guidelines about the number of holes needed to soften the core of coal specimens of different $\mathrm{W} / \mathrm{H}$ ratios. Prassetyo (2010) studied experimentally in the laboratory the variation of the "core zone" at different width-to-height ratios and concluded that the higher the width-to-height ratio, the bigger 
the core zone. Hence the bigger the core zone, the higher the number of holes needed to soften that core. Therefore, for small width- to-height ratio such as 2 , only one hole at the center of the specimen might be enough to soften the core. However, for higher width-toheight ratios such as 3 and 4, more holes are needed. To make the distribution of the holes symmetric with respect to the cross-section of the coal specimen, five holes will be used for both width-to-height ratios 3 and 4 as shown in Figure 6-4.

Coal specimens of square cross-section and width-to-height ratio equals to 2,3 , and 4 have been tested under strain control of $10^{-4} \mathrm{in} / \mathrm{sec}$. The interface friction between the loading platens and the end surfaces of coal specimens is assumed to be 0.25 . The height of the specimen was one inch.

Table 6-2 shows the test results for the coal specimens where the core zone was softened by vertical holes of diameter one-sixteenth of an inch and 0.8 in deep. The tangent Young's modulus of the coal specimens was determined at $50 \%$ of their ultimate strength. All coal specimens failed non-violently without sudden loss of strength and the amount of ejection and the degree of noise during failure of coal specimen were very low. This was because the strength of the coal specimens decreased significantly when the core was softened as compared with other coal specimens without softening. Compare the UCS values in Table 3-1 where the coal specimens were not softened with those in Table 6-2 where the core of the coal specimens was softened. The percentage reduction in the average UCS for coal specimens of different width-to-height ratios due to softening the core is shown in Table 6-3. It is obvious that the reduction approaches $20 \%$.

Hence the gradual yielding of core zone eliminates the violent failure because drilling holes reduces the horizontal stresses. Moreover, the core zone will not store much 
energy since it can expand laterally due to the existence of the drilled holes and part of the input energy will be consumed in closing these holes. As a result, coal specimens with core softening fail non-violently, while those without core softening fail violently especially at $\mathrm{W} / \mathrm{H}$ ratio $=4$.

Maleki (1995) reported in his analysis of four bump prone mines that the induced horizontal stress was one of the common factors noticed in these mines. He also noticed that yielding of the floor material reduced the horizontal stresses in the pillar and enhanced gradual pillar failure. That was exactly the basic role that the vertical holes played. Since all coal specimens in Table 6-2 failed non-violently. It is assumed that coal pillars of W/H ratio 2,3 , and 4, by analogy, would not fail violently as long as their strength did not exceed the average strength listed in Table 6-2, because all coal specimens shown in Table 6-2 failed non-violently. 
Table 6-2 Test results for coal specimens of various width-to-height ratios where the core was softened by vertical holes

\begin{tabular}{|c|c|c|c|c|c|c|c|c|c|c|c|c|}
\hline $\begin{array}{l}\text { W/H } \\
\text { ratio }\end{array}$ & $\begin{array}{c}\text { Specimen } \\
\#\end{array}$ & $\begin{array}{c}\text { Vertical } \\
\text { strength, psi }\end{array}$ & $\begin{array}{c}\text { Vertical } \\
\text { strength }_{\text {avg }}, \\
\text { psi }\end{array}$ & $\begin{array}{c}\text { Vertical } \\
\text { Strength }_{\text {avg }}, \\
\text { psi }\end{array}$ & E, psi & $\overline{\mathbf{E}_{\text {avg }}, \mathrm{psi}}$ & $\begin{array}{c}\text { E-std., } \\
\text { psi }\end{array}$ & $\begin{array}{c}\text { Failure } \\
\text { type }\end{array}$ & Ejection & Noise & $\begin{array}{c}\begin{array}{c}\text { Sudden } \\
\text { loss } \\
\text { of }\end{array} \\
\text { strength }\end{array}$ & $\begin{array}{c}\text { Hole } \\
\text { depth, } \\
\text { in }\end{array}$ \\
\hline \multirow{4}{*}{2} & 1 & 5,039 & \multirow{4}{*}{4,709} & \multirow{4}{*}{318} & 313,818 & \multirow{4}{*}{301,508} & \multirow{4}{*}{24,645} & $\begin{array}{c}\text { Non- } \\
\text { violent }\end{array}$ & Low & Low & No & 0.8 \\
\hline & 2 & 4,890 & & & 326,988 & & & $\begin{array}{c}\text { Non- } \\
\text { violent }\end{array}$ & Low & Low & No & 0.8 \\
\hline & 3 & 4,579 & & & 270,217 & & & $\begin{array}{c}\text { Non- } \\
\text { violent }\end{array}$ & Low & Low & No & 0.8 \\
\hline & 4 & 4,328 & & & 295,009 & & & $\begin{array}{c}\text { Non- } \\
\text { violent }\end{array}$ & Low & Low & No & 0.8 \\
\hline \multirow{3}{*}{3} & 1 & 5,430 & \multirow{3}{*}{5,210} & \multirow{3}{*}{438} & 216,105 & \multirow{3}{*}{227,373} & \multirow{3}{*}{44,046} & $\begin{array}{c}\text { Non- } \\
\text { violent }\end{array}$ & Low & Low & No & 0.8 \\
\hline & 2 & 5,494 & & & 275,958 & & & $\begin{array}{c}\text { Non- } \\
\text { violent }\end{array}$ & Low & Low & No & 0.8 \\
\hline & 3 & 4,705 & & & 190,055 & & & $\begin{array}{c}\text { Non- } \\
\text { violent }\end{array}$ & Low & Low & No & 0.8 \\
\hline \multirow{4}{*}{4} & 1 & 6,441 & \multirow{3}{*}{6,362} & \multirow{3}{*}{201} & 213,474 & \multirow{3}{*}{211,967} & \multirow{3}{*}{9,609} & $\begin{array}{c}\text { Non- } \\
\text { violent }\end{array}$ & Low & Low & No & 1.0 \\
\hline & 2 & 6,511 & & & 220,734 & & & $\begin{array}{c}\text { Non- } \\
\text { violent }\end{array}$ & Low & Low & No & 1.0 \\
\hline & 3 & 6,133 & & & 201,693 & & & $\begin{array}{c}\text { Non- } \\
\text { violent }\end{array}$ & Low & Low & No & 1.0 \\
\hline & 4 & 7,493 & 7,290 & 618 & 236,223 & 220,872 & 15,805 & $\begin{array}{c}\text { Non- } \\
\text { violent }\end{array}$ & Low & Low & No & 0.8 \\
\hline
\end{tabular}




\begin{tabular}{|c|c|c|c|c|c|c|c|}
\hline 5 & 7,576 & 235,266 & $\begin{array}{l}\text { Non- } \\
\text { violent }\end{array}$ & Low & Low & No & 0.8 \\
\hline 6 & 6,055 & 191,028 & $\begin{array}{l}\text { Non- } \\
\text { violent }\end{array}$ & Low & Low & No & 0.8 \\
\hline 7 & 7,906 & 218,542 & $\begin{array}{c}\text { Non- } \\
\text { violent }\end{array}$ & Low & Low & No & 0.8 \\
\hline 8 & 7,010 & 218,979 & $\begin{array}{c}\text { Non- } \\
\text { violent }\end{array}$ & Low & Low & No & 0.8 \\
\hline 9 & 7,533 & 226,140 & $\begin{array}{c}\text { Non- } \\
\text { violent }\end{array}$ & Low & Low & No & 0.8 \\
\hline 10 & 6,874 & 206,652 & $\begin{array}{c}\text { Non- } \\
\text { violent }\end{array}$ & Low & Low & No & 0.8 \\
\hline 11 & 7,872 & 234,147 & $\begin{array}{c}\text { Non- } \\
\text { violent }\end{array}$ & Low & Low & No & 0.8 \\
\hline
\end{tabular}




\section{Table 6-3 Percent reduction in UCS for coal specimens with and without core softening}

\begin{tabular}{|c|c|}
\hline W/H ratio & Reduction in UCS, \% \\
\hline $\mathbf{2}$ & -19.6 \\
\hline $\mathbf{3}$ & -19.2 \\
\hline $\mathbf{4}$ & -17.0 \\
\hline
\end{tabular}

Figure 6-5 compares the stress-strain curves for some coal specimens of $\mathrm{W} / \mathrm{H}$ ratio $=$ 4 with and without core softening. Solid lines denote coal specimens with core softening, while dotted lines in the post-peak region represent those without core softening. It is obvious that the coal specimens with core softening do not lose their strength suddenly after the peak strength is reached. They keep deforming after having reached the peak strength. While coal specimens without core softening lose their strength more suddenly and completely in a violent way when the peak strength is reached and there is almost no plastic deformation for those specimens. Moreover, the post-peak slope of the stress strain curve of the specimens with core softening is smaller than those without core softening; please compare the angles $\beta_{1}$ with $\beta_{2}$. When the post-peak slope is high, the rate of stiffness degradation is higher and the crack propagates faster and hence the time period to complete failure is much shorter. Conversely, when the post-peak slope is small, crack propagation is slow, and the time period for complete failure is longer. So drilling theses vertical holes in the core zone delays the crack propagation and converts the failure from brittle to ductile. According to the local mine stiffness criteria introduced by Salamon (1969), which is used to distinguish between stable and unstable pillar failure, the coal specimens with core softening are more stable than those without core softening, because the post-peak slope for coal specimen with core softening is less than those without core softening. 


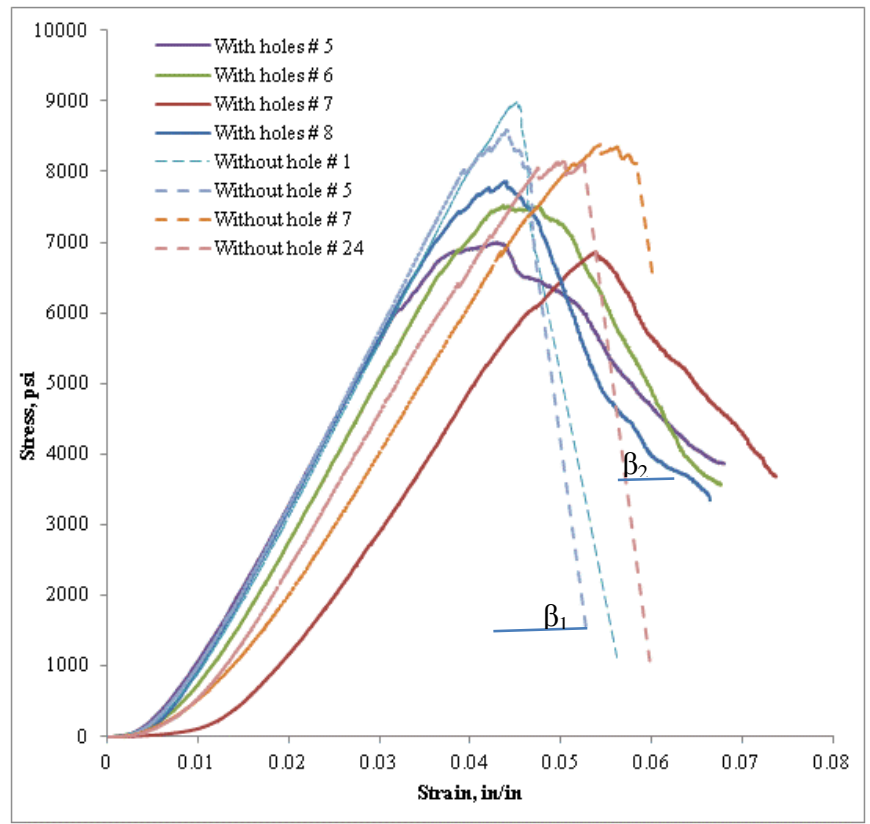

Figure 6-5 Stress-strain curves for coal specimens of $\mathrm{W} / \mathrm{H}$ ratio $=4$ with and without core softening

Figure 6-6 shows some coal specimens of width-to-height ratio $=4$ before and after failure. A very thin wire was used to check whether the drilled holes of the failed coal specimens were closed or not. For coal specimen of width-to-height ratio 2, there was no obvious closure for the drilled holes. Since the lateral deformation was a fraction of the vertical deformation and the vertical deformation was small, so the lateral deformation was insignificant. However, for coal specimen of $\mathrm{W} / \mathrm{H}$ ratio $=3$ and 4 , it was found that the holes near the center of some coal specimens were almost closed while the others were partially closed. The closure of drilled holes meant lateral expansion and deformation of the core of the coal specimen. This lateral expansion consumed some of the input energy and reduced the violent failure. 

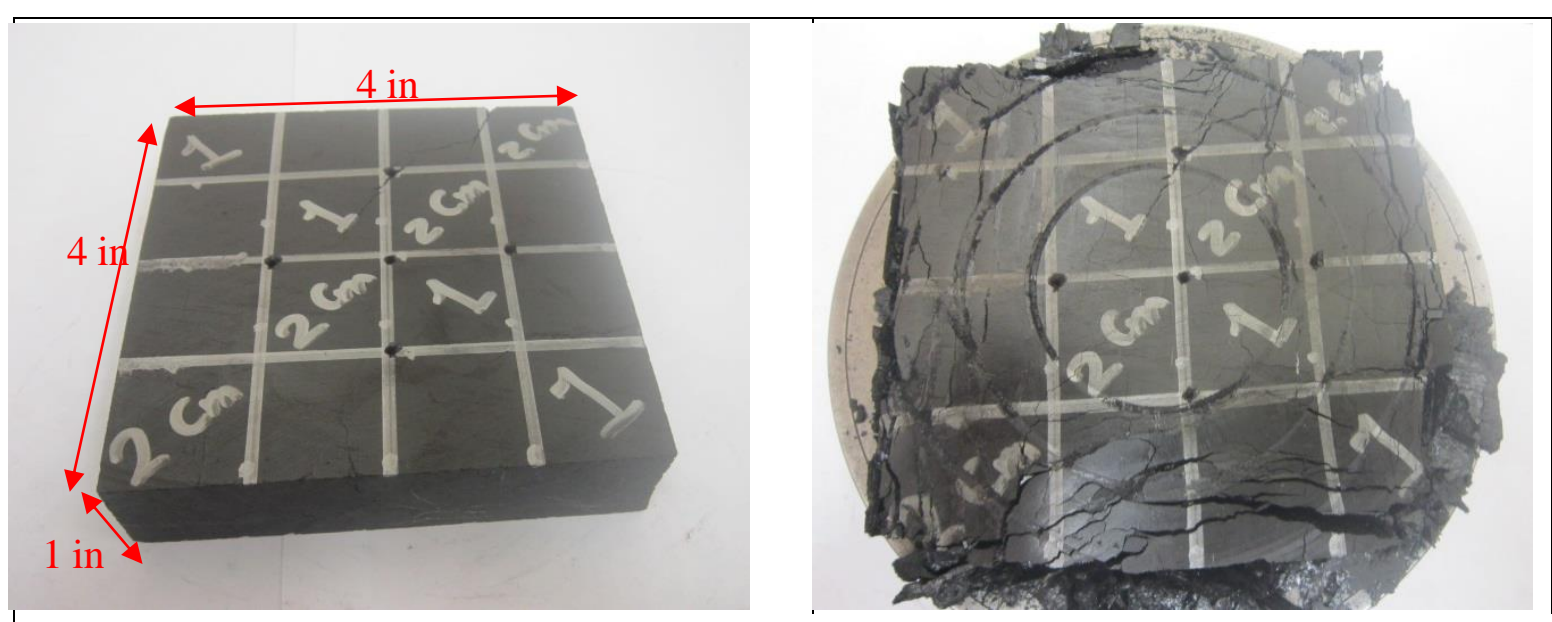

Sample \# $1, \mathrm{~W} / \mathrm{H}=4$, please note the closure of hole diameter for the failed specimen.

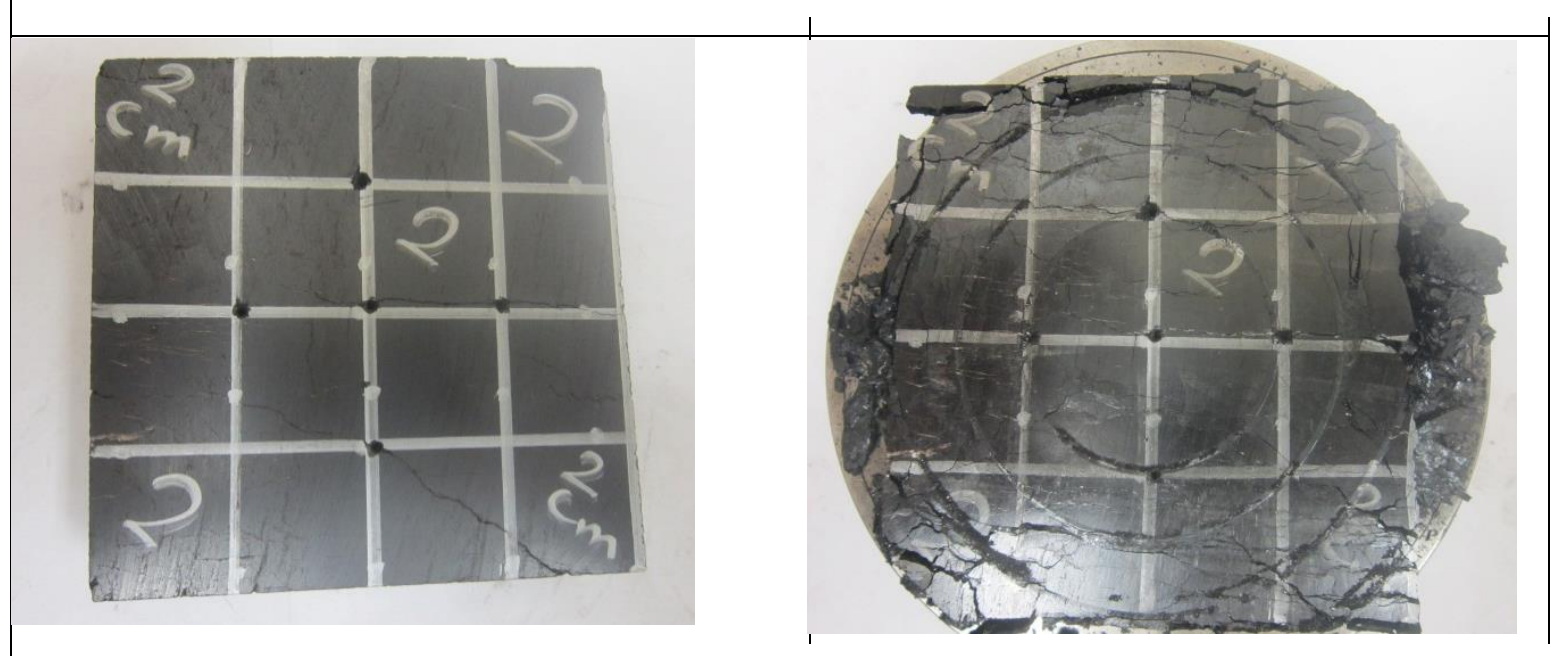

Sample \# 2, W/H = 4, please note the shrinkage in the hole diameter for the failed specimen.

Figure 6-6 Examples of Coal specimens of $\mathrm{W} / \mathrm{H}$ ratio= 4 with core softening before and after failure

\subsection{Testing the Third Hypothesis to Mitigate the Violent Failure}

In this section, an attempt was made to mitigate the violent failure of coal specimens through softening the coal specimens completely by immersing them in water for 24 hours, then air-dried for one hour, and finally tested under strain control of $10^{-4}$ in/sec. 
Table 6-4 shows the test results for coal specimens of width-to-height ratio 3 and 4. The height of these coal specimens were kept constant at 1 in. It is obvious from Table 6-4 that softening the coal specimens completely by immersing them in water was effective in reducing violent failure of coal specimen of width-to-height ratios 3 and 4 . There was no sudden loss of strength, and the degree of ejection for most of the coal specimens was low. Water infusion was one of the de-stressing method used to destroy the structural integrity of coal so that it cannot store sufficient energy to fail violently had been used previously (Peng, 2008). Campoli (1987) stated that the pillars that were de-stressed through water infusion were mined without incidents. 
Table 6-4 Test results for coal specimens of width-to-height ratios equals to 3 and 4 softened completely by water

\begin{tabular}{|c|c|c|c|c|c|c|c|c|c|c|c|}
\hline $\begin{array}{l}\mathrm{W} / \mathrm{H} \\
\text { ratio }\end{array}$ & $\begin{array}{c}\text { Specimen } \\
\#\end{array}$ & $\begin{array}{c}\text { Vertical } \\
\text { strengt, } \\
\text { psi }\end{array}$ & $\begin{array}{c}\text { Vertical } \\
\text { strength- } \\
\text { avg, psi }\end{array}$ & $\begin{array}{c}\text { Vertical } \\
\text { stress- } \\
\text { std., psi }\end{array}$ & E, psi & $\begin{array}{l}\mathbf{E}_{\text {Avg }} \\
, \text { psi }\end{array}$ & $\begin{array}{c}\text { E-std., } \\
\text { psi }\end{array}$ & Failure type & Ejection & Noise & $\begin{array}{c}\text { Sudden } \\
\text { loss of } \\
\text { strength }\end{array}$ \\
\hline \multirow{6}{*}{3} & 2 & 6,121 & \multirow{6}{*}{6,065} & \multirow{6}{*}{886.54} & 295,352 & \multirow{6}{*}{280,139} & \multirow{6}{*}{25,756} & Non-violent & Low & Moderate & No \\
\hline & 3 & 5,369 & & & 249,377 & & & Non-violent & Low & Low & No \\
\hline & 4 & 5,114 & & & 256,072 & & & Non-violent & Low & Low & No \\
\hline & 5 & 6,315 & & & 268,183 & & & Non-violent & Low & Low & No \\
\hline & 6 & 7,623 & & & 312,534 & & & Non-violent & High & high & No \\
\hline & 7 & 5,846 & & & 299,313 & & & Non-violent & Moderate & Moderate & No \\
\hline \multirow{8}{*}{4} & 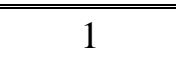 & 6,889 & \multirow{8}{*}{7,949} & \multirow{8}{*}{1,152} & 242,964 & \multirow{8}{*}{238,854} & \multirow{8}{*}{24,62} & Non-violent & Low & Moderate & No \\
\hline & 2 & 7,316 & & & 220,247 & & & Non-violent & Low & Moderate & No \\
\hline & 3 & 8,071 & & & 233,832 & & & Non-violent & Low & Moderate & No \\
\hline & 4 & 8,380 & & & 278,327 & & & Non-violent & High & Moderate & No \\
\hline & 6 & 8,350 & & & 236,706 & & & Non-violent & V. high & V. high & No \\
\hline & 7 & 9,619 & & & 237,273 & & & Non-violent & Low & Low & No \\
\hline & 8 & 6,034 & & & 10197,876 & & & "Non-violent & Low & Moderate & No \\
\hline & 9 & 8,929 & & & 263,604 & & & Non-violent & Low & Low & No \\
\hline
\end{tabular}


By comparing the test results in Table 3-1 where the coal specimens did not experience any softening at all with the test results in Table 6-4 where the coal specimens were softened with water, the average vertical strength decreases $6 \%$ and $10 \%$ for coal specimens of width-to-height ratios $=3$ and 4 , respectively. The reduction in the average vertical strength may be attributed to the pore water pressure that built up inside the pores of the coal specimen.

Therefore, it is quite obvious that softening the core zone was the most effective technique compared to softening the ribs, or softening the whole specimens with water; because there was no sudden loss of strength and both the ejection and the noise during failure were low for all coal specimens of different $\mathrm{W} / \mathrm{H}$ ratios.

\subsection{Summary for Chapter Six}

Three hypotheses have been studied in chapter six to investigate mitigating the violent failure of coal specimens. The three hypotheses depend on softening coal specimens either partially or completely. Softening coal specimens partially occurs through drilling small holes either in the ribs or the core zone. However, softening coal specimens completely occurs through immersing them in water for 24 hours and directly testing them.

It was found that the most effective way to mitigate the violent failure of coal specimen is to soften their core zones. Both the sound level (noise) at failure and the ejections reduced significantly when the core zone was softened. This gives an indication that the elastic energy stored in the core zone of the coal specimens is the main cause for violet failure, and to avoid bump, the structural integrity of the core zone has to be broken. 


\section{CHAPTER 7: FAILURE OF A SIMULATED LAYERED ROCK SPECIMEN}

\subsection{Background}

A variety of geological factors control the occurrence of bumps. A common factor in both U.S. and foreign coal mines is the proximity of the coal seam to strong, thick, and rigid strata (lannacchione and Zelanko, 1994; Peng, 2008). Iannacchione and Zelanko (1995) stated that among 95 sites reviewed, in 86 instances reference is made to the presence of sandstone immediately above or within a few meters of the coal seam. Agapito et.al., (1997) observed that stability problems at Deer Creek were mostly due to coal bursts caused by stresses associated with deep cover and sandstone channels. The uniaxial compressive strength of this sandstone showed values as high as 24,500 psi. The thickness of Castlegate Sandstone is approximately $200 \mathrm{ft}$. The Young's modulus ratio for roof / coal is approximately 20 .

Since bump prone coal seams are often overlain by a strong and stiff stratum or strata such as sandstone or massive shale. It is necessary to answer the following question: Under static loading conditions, is the existence of sandstone, or strong rock, enough for a burst to occur? Furthermore, does the mechanics of failure change with changing the strength of the roof and floor?

To answer these questions, man-made concrete specimens are assembled in a three layered approach; the top layer simulates the roof, while the middle and the bottom layers simulate the pillar and the floor, respectively. 
Chapter seven is divided into three phases: Phase one includes the development of the simulated materials and the definition of the rock properties of strong, medium and weak. This phase will include the material properties of the simulated samples. Phase two, includes testing the man-made concrete specimens and Phase three includes the results \& discussions.

\subsection{Methodology in Chapter Seven}

It is obvious from documented cases of bumps that the geological environment surrounding coal pillar and in particular the interaction between pillar/roof \& pillar/floor plays a role in coal bumps. One approach for furthering the understanding of the mechanisms associated with violent failures in underground mines is the testing of simulated rock conditions (man-made concrete specimens) in a laboratory setting. These man-made concrete specimens will be of different properties (unconfined compressive strength) and will be assembled in a three layered form. These 3 layers represent roof, pillar and floor. Hence, different geological or field conditions can be simulated through changing the strength of these three layers.

To be more precise, eight different geological or field conditions have been investigated through changing or controlling the UCS of these three layers. The reason behind using man-made concrete specimens rather than actual rock and coal specimens is that, actual coal and rock experience huge variation in strength depending on their environment and geological conditions. For instance, when some specimens are selected from the same coal seam, the same mine, and same location, it is expected that their strength will not be the same. This point has been proved in chapter two by testing 20 coal specimens of the Sunnyside coal seam from the West Ridge Mine, East Carbon, Utah. 
Therefore, the ability to examine and determine a specific geologic condition contributes to bump using actual coal and rock specimens is not possible, because there is uncertainty about their strength. Furthermore, the strength of the man-made concrete specimens is well known even before running the tests.

\subsection{Assessment of Violent Failure}

Since coal bumps are always accompanied by loud audible noise, sound pressure level (SPL) meter can be used to assess the degree of violence. SoundPro SE/DL instrument was used to analyze sound signals over the full bandwidth of the instrument which referred to as "broadband measurement". Moreover the stress-strain curve will be examined to see whether there is a sudden loss of strength or not. Therefore, sudden loss of strength and the Peak SPL are the criteria to judge whether the failure is violent or not in chapter seven.

\subsection{Phase One "Definition of Coal and Rock Strength"}

It is expected that a bump would occur when specific field conditions are encountered. Therefore, in order to simulate those particular conditions conducive to bump in the lab, there should be no doubt about coal and rock strength because of the variation in the rock and coal strength. In other words, it is necessary to pre-determine the mechanical properties of coal and rock surrounding that coal pillar before conducting the laboratory tests.

Man-made concrete specimens having the same properties as coal and rock are used to simulate different geologic conditions. The specimens are of different UCS and will 
be assembled in three layers. Eight different geological or field conditions are modeled in this research. Summary of these 8 groups is shown in Table 7-1.

Table 7-1 Eight combinations of simulated geological conditions

\begin{tabular}{|c|c|c|c|}
\hline Group \# & $\begin{array}{l}\text { Roof } \\
\text { Layer) }\end{array}$ & (Top & $\begin{array}{l}\text { Pillar } \\
\text { Layer) }\end{array}$ \\
\hline $\mathbf{1}$ & Strong & Medium & $\begin{array}{l}\text { Floor } \\
\text { Layer) }\end{array}$ \\
\hline $\mathbf{2}$ & Strong & Strong & Strong \\
\hline $\mathbf{3}$ & Weak & Medium & Strong \\
\hline $\mathbf{4}$ & Weak & Strong & Strong \\
\hline $\mathbf{5}$ & Strong & Strong & Weak \\
\hline $\mathbf{6}$ & Strong & Medium & Weak \\
\hline $\mathbf{7}$ & Weak & Medium & Weak \\
\hline $\mathbf{8}$ & Weak & Strong & Weak \\
\hline
\end{tabular}

\subsubsection{Definition of Coal Strength}

The man-made concrete specimens are the substitute for the actual coal and rock specimens. The strength of the man-made concrete specimens should be similar to the strength of the coal and rock specimens. In other words, the unconfined compressive strength (UCS) of the concrete specimens with three layers simulating roof, coal pillar and floor has to be very close to that of coal and rock specimens. In this research the coal pillar is assumed to be either of medium or strong strength. Therefore, a question is raised "what is the UCS for medium or strong coal pillar?" To define precisely the strength of coal, it is better to go back to Chapter three and specifically to Table $3-4$, where twenty cylindrical coal samples of height to diameter $(\mathrm{H} / \mathrm{D})$ ratio $=2$ from the Sunnyside coal seam have been tested. The same table is shown here again as Table 7-2 for convenience. 
Table 7-2 UCS test results for 20 cylinder coal specimens of the Sunnyside seam

\begin{tabular}{|c|c|c|c|c|c|c|c|c|c|c|c|}
\hline $\begin{array}{l}\text { Sampl } \\
\text { e \# }\end{array}$ & $\begin{array}{l}\text { H, } \\
\text { in }\end{array}$ & $\begin{array}{l}\text { D, } \\
\text { in }\end{array}$ & $\begin{array}{l}\text { H/D } \\
\text { ratio }\end{array}$ & $\mathbf{l b} / \mathrm{ft}^{3}$ & $\begin{array}{l}\text { UCS, } \\
\text { psi }\end{array}$ & $\begin{array}{l}\text { UCS }_{\text {avg, }} \\
\text { psi }\end{array}$ & $\begin{array}{l}\mathbf{U C S}_{\text {std }} \\
\text { psi }\end{array}$ & $\begin{array}{l}\text { E- } \\
\text { tangent } \\
\text { psi }\end{array}$ & $\begin{array}{l}\mathbf{E}_{\text {av }} \\
\text { g } \\
\text { psi }\end{array}$ & $\begin{array}{l}\mathbf{E}_{\text {std. }} \\
\text { psi }\end{array}$ & $\begin{array}{l}\text { remark } \\
\mathrm{S}\end{array}$ \\
\hline 1 & 3.957 & 1.995 & 1.98 & -- & 4680 & \multirow[t]{12}{*}{3381} & \multirow[t]{12}{*}{797} & 536504 & \multirow[t]{12}{*}{459018} & \multirow[t]{12}{*}{70447} & \multirow{12}{*}{$\begin{array}{l}\text { Samples } \\
\text { Without } \\
\text { Macro- } \\
\text { cracks }\end{array}$} \\
\hline 2 & 3.88 & 1.995 & 1.94 & - & 2641 & & & 423934 & & & \\
\hline 3 & 3.972 & 1.994 & 1.99 & -- & 2829 & & & 471577 & & & \\
\hline 4 & 3.978 & 1.998 & 1.99 & 79.15 & 2926 & & & 480165 & & & \\
\hline 5 & 4.058 & 1.986 & 2.04 & 78.05 & 3594 & & & 505001 & & & \\
\hline 6 & 4.09 & 1.994 & 2.05 & 78.78 & 2921 & & & 402984 & & & \\
\hline 7 & 3.992 & 1.997 & 2.00 & 78.18 & 2536 & & & 378125 & & & \\
\hline 8 & 4.074 & 1.992 & 2.05 & 78.40 & 4444 & & & 507081 & & & \\
\hline 9 & 4.096 & 1.993 & 2.06 & 78.40 & 3076 & & & 443147 & & & \\
\hline 10 & 3.952 & 1.997 & 1.98 & 79.00 & 2537 & & & 307248 & & & \\
\hline 11 & 3.988 & 1.998 & 2.00 & 78.22 & 4137 & & & 538945 & & & \\
\hline 12 & 4.014 & 1.986 & 2.02 & 78.43 & 4252 & & & 513501 & & & \\
\hline 13 & 3.97 & 1.986 & 2.00 & 77.70 & 2237 & \multirow[t]{2}{*}{2280} & \multirow[t]{2}{*}{299} & 267827 & \multirow[t]{2}{*}{343755} & \multirow[t]{2}{*}{58292} & \multirow{2}{*}{$\begin{array}{l}\text { Samples } \\
\text { with } \\
\text { macro- } \\
\text { cracks }\end{array}$} \\
\hline 14 & 4.044 & 1.993 & 2.03 & 78.22 & 2590 & & & 300538 & & & \\
\hline
\end{tabular}




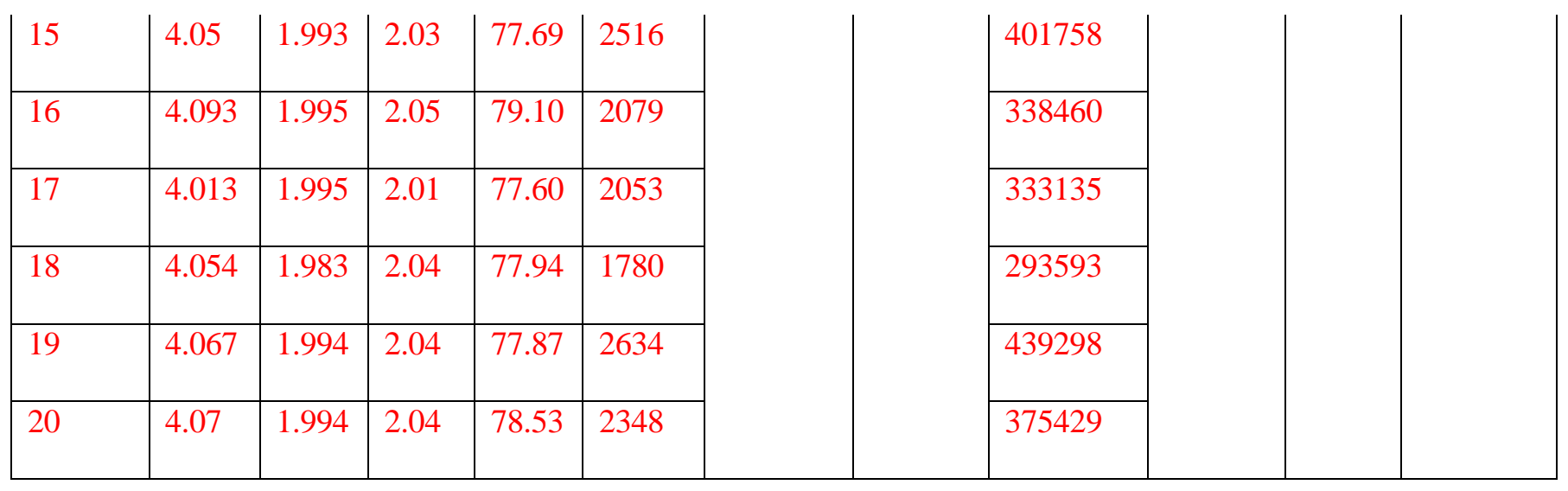


The UCS test results for the twenty coal specimens from Utah's Sunnyside seam range from 1,780 to $4,680 \mathrm{psi}$. This range is expected to cover coal strength probably from weak to very strong. Figure 7-1 shows the probability distribution for the UCS test results. The summary statistics for the test results are shown in Table 7-3.

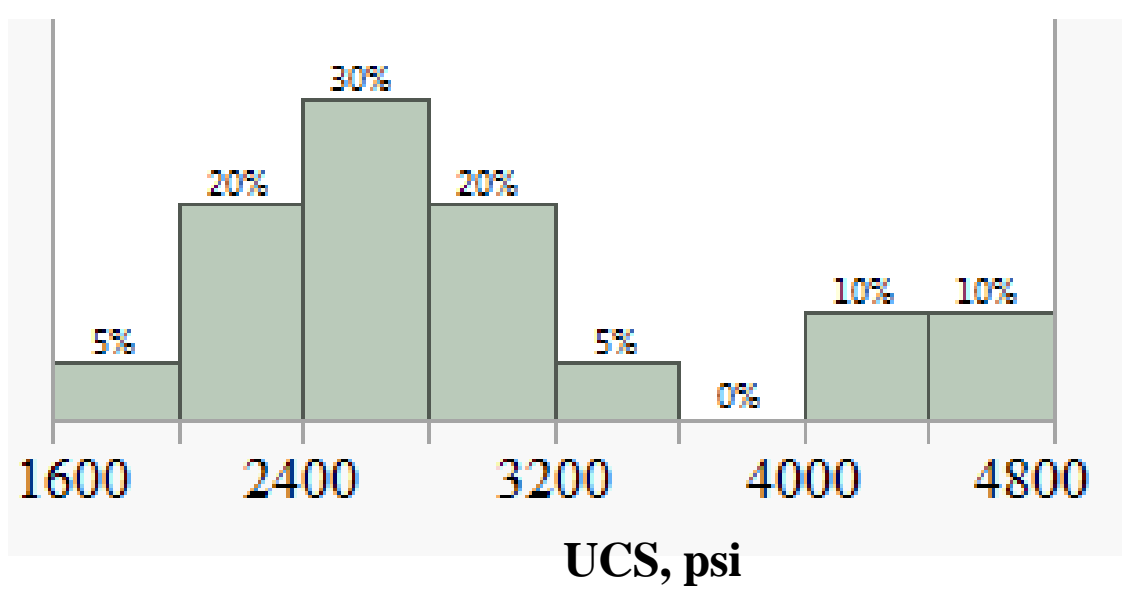

Figure 7-1 Probability distribution for UCS test results from 20 coal specimens

Although these coal specimens have been taken from the same coal seam (Sunnyside coal seam) and the same mine, and probably the same location, a significant range has been noticed in coal strength where the standard deviation is 840 psi. Furthermore, the maximum value of the coal strength is 2.7 times the minimum value. The mean value for the 20 specimens is 1,491 psi, and the strength of $50 \%$ of coal specimens ranges from 2,400 psi to 3,200 psi. Twenty percent of the coal specimens have UCS ranging from 4,000 to 4,680. It is assumed that the UCS from 2,000 to 2,400 psi represents the medium strength, while UCS from 4,000 to 4,680 is strong strength.

2,000 and 4,000 psi have been assumed to represent the medium and strong coal pillars, respectively. Hence, whenever in chapter seven a medium strength coal pillar is mentioned, its UCS is assumed to be 2,000 psi, and whenever a strong coal pillar is 
mentioned, its strength is assumed to be 4,000 psi. So, basically, the assumed UCS for a strong pillar is two times the medium one.

Table 7-3 Summary statistics for UCS tests for 20 cylindrical coal specimens from Utah's Sunnyside seam

\begin{tabular}{|c|l|c|}
\hline Quartile & & UCS, psi \\
\hline $100.00 \%$ & maximum & 4680 \\
\hline $99.50 \%$ & & 4680 \\
\hline $97.50 \%$ & & 4680 \\
\hline $90.00 \%$ & & 4425 \\
\hline $75.00 \%$ & quartile & 3465 \\
\hline $50.00 \%$ & median & 2638 \\
\hline $25.00 \%$ & quartile & 2390 \\
\hline $10.00 \%$ & & 2056 \\
\hline $2.50 \%$ & & 1780 \\
\hline $0.50 \%$ & & 1780 \\
\hline $0.00 \%$ & minimum & 1780 \\
\hline Mean & & 2941 \\
\hline Std. Dev. & & 840.8 \\
\hline & & \\
\hline
\end{tabular}

\subsubsection{Definition of Rock Strength}

In order to develop the specified eight groups shown in Table 2.1 it is necessary to define what do strong, medium and weak strength mean for rocks. Table 7-4 shows the classification of rock strength for intact rock specimens. This classification is based on the uniaxial compressive strength recommended by the international society of Rock Mechanics (ISRM) and based on Brown, 1981. 
Table 7-4 Classification of UCS for intact rock (Recommendations from ISRM, based on Brown, 1981)

\begin{tabular}{|c|c|c|}
\hline Description & UCS, MPa & UCS, psi from \\
\hline extremely weak & $<1$ & $36.3-145.0$ \\
\hline very weak & $1-5$ & $145.0-725.2$ \\
\hline weak & $5-25$ & $725.2-3,625.9$ \\
\hline medium strong & $25-50$ & $3625.9-7,25.9$ \\
\hline strong & $50-100$ & $7251.9-14,503.7$ \\
\hline very strong & $100-250$ & $14503.7-36,259.3$ \\
\hline extremely strong & $>250$ & $36,259.3$ \\
\hline
\end{tabular}

A strong rock ranges from 7,251 to 14,503 psi, while a weak rock ranges from 725 to 3,625 psi according to ISRM shown in Table 7-4. In chapter seven, the strong rock involved in the man-made concrete specimens is assumed to have 9,000 psi, while weak rock is assumed to have $4,000 \mathrm{psi}$. Hence the strength of strong rock is more than two times the weak rock. Table 7-5 summarizes the recommended UCS values for the man-made concrete specimens used to simulate coal pillar and rocks. For coal, 2,000 and 4,000 psi represent medium and strong coal pillar or concrete specimens, respectively, while for rock, 4,000 and 9000 psi represent weak and strong rock, respectively.

Table 7-5 Recommended UCS values for coal pillars and rock

\begin{tabular}{|c|c|c|}
\hline Description & Coal pillar & Rock (roof or floor) \\
\hline Weak & Not included & $4,000 \mathrm{psi}$ \\
\hline Medium & $2,000 \mathrm{psi}$ & Not included \\
\hline Strong & $4,000 \mathrm{psi}$ & $9,000 \mathrm{psi}$ \\
\hline
\end{tabular}




\subsection{Phase One "Development of Man-made Concrete Specimens"}

The strength of "conventional concrete" is smaller than that of "high strength concrete". That's why the principles of high strength concrete mix design have been employed in this research to obtain a concrete specimen of strength 9,000 psi to simulate strong rock such as sandstone. On the other hand, the principles of conventional concrete mix design have been applied to obtain a medium strength or strong pillar or strong pillar or weak rock. The strength of concrete is governed by the quantity of mixing water used per unit of cement or cementing material (Abrams, 1918)

\subsubsection{Sample Preparation}

Before designing a concrete mixture of specific strength, the selection of proper component materials and their proportions is the first step toward obtaining the required specified strength. The factors that are important from the standpoint of concrete strength are considered here.

\section{i. Concrete}

The two major components of concrete are a cement paste and inert materials. The cement paste consists of Portland cement, water, and some air either in the form of naturally entrapped air voids or minute, intentionally entrained air bubbles. The inert materials are usually composed of fine aggregate such as sand, and coarse aggregate such as gravel, crushed stone, or slag. When Portland cement is mixed with water, the compounds of the cement react to form a cementing medium. In a properly mixed concrete, each particle of sand and coarse aggregate is completely surrounded and coated and all spaces between the particles are filled with the paste. As the cement paste sets and hardens, it binds the aggregates into a solid mass. Under normal conditions, the concrete grows 
stronger as it grows older. The chemical reactions between cement and water that cause the paste to harden and bind the aggregates together require time. The reactions take place very rapidly at first and then more slowly over a long period of time (Caldarone, 2009).

\section{ii. Cement}

Cement is a material that has adhesive and cohesive properties enabling it to bond mineral fragments into a solid mass. Cement consists of silicates and aluminates of lime made from limestone and clay (or shale) which is ground, blended, fused in a kiln and

crushed to a powder. Cement chemically combines with water (hydration) to form a hardened mass (Wang and Salamon, 1979).

\section{iii. Water}

The water has two roles in concrete mixture: First is to perform cement hydration and second is to make the concrete composition fluid and workable. The water which is used to make the concrete is the tap water.

\section{iv. Water-Cement Ratio}

Figure 7-2 shows approximate relationship between compressive strength and water to cementing materials (W/C) ratio for concrete using $19-\mathrm{mm}$ to $25-\mathrm{mm}$ nominal maximum size coarse aggregate. Basically, as the water to cementitious material (W/C) ratio increases, the compressive strength decreases. Hence, the specific strength of concrete specimens can be achieved through changing the $\mathrm{W} / \mathrm{C}$ ratio. 


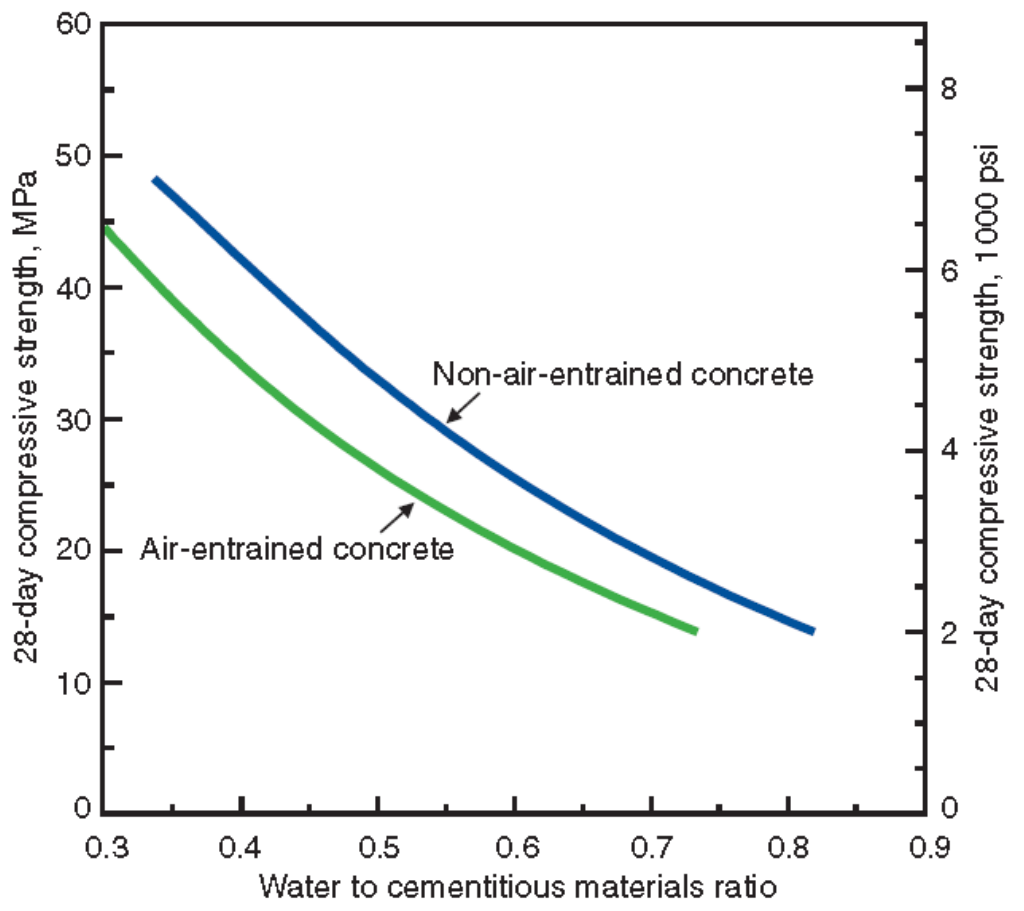

Figure 7-2 Variation of UCS with changing W/C ratio (Mehta and Monteiro, 2006)

\section{v. Aggregate}

The aggregate strength is usually not a factor in the strength of conventional concrete, because the aggregate particles are several times stronger than the matrix and the interfacial transition zone in concrete. It may be anticipated that, independent of the waterto-cement (W/C) ratio, the size, shape, surface texture, and mineralogy of aggregate particles would affect concrete strength. With the same cement content and consistency, concrete mixtures containing larger aggregate particles require less mixing water than those containing smaller aggregate. On the contrary, larger aggregates tend to form weaker interfacial transition zones containing more micro-cracks. 


\section{vi. Admixtures}

High strength concrete used to simulate strong rock is characterized by small waterto-cement (W/C) ratio. The workability of concrete would be too low due to the small W/C ratio, hence, water-reducing admixtures has to be used. Furthermore, water-reducing admixtures can enhance the ultimate strength of concrete. At given water-to-cement (W/C) ratio, the presence of water-reducing admixtures in concrete generally has a positive influence on the rates of cement hydration and early strength development.

Rheomac SF 100 Silica Fume (BASF Chemical-website \#1) mineral admixture was used in the design of high strength concrete, which was used to simulate strong rock such as sandstone. The Silica Fume produces extremely strong, durable concrete and decreases permeability. Moreover, DELVO STABILIZER (BASF chemical website\#2) which is a hydration control agent was used to improve workability and increase the ultimate strength of the concrete specimens. Furthermore, it retards the setting time by controlling the hydration of Portland cement.

\section{vii. Curing Conditions}

When the Portland cement is mixed with water, a chemical reaction called hydration takes place. Curing can be defined as a procedure for insuring the hydration of the Portland cement in newly-placed concrete. Figure 7-3 shows the curing process for the man-made concrete specimens used to simulate the eight different field conditions. The concrete specimens are immersed in water for curing.

The main objective of concrete curing is to increase its strength and lessen the chance of concrete cracking due to shrinkage. This is because hydration can proceed 
satisfactorily only under conditions of saturation. More details about the concrete mix proportions for these specimens will be presented later in this chapter.

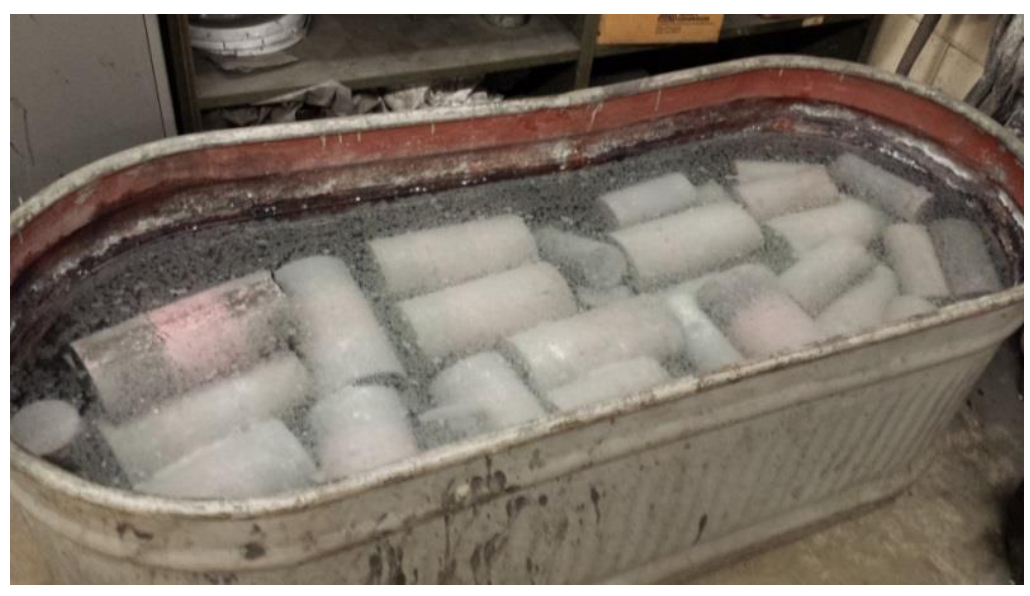

Figure 7-3 Curing of concrete specimens

\subsection{Trial Batches}

At the same water-to-cement (W/C) ratio and using the same type of cement and chemical admixtures, differences in concrete strength may result from other factors, such as (1) changes in the aggregate size, grading, surface texture, shape, strength and stiffness; (2) entrained-air content; (3) the length of curing time (Mehta and Monteiro, 2006). Hence, designing a concrete mix of a specific strength to simulate rock strength is a combination of art and science. Therefore, trial batches were prepared first to obtain guidelines about the proportions of a concrete mix to obtain different rock and coal strengths.

\subsubsection{Trial Batches for Conventional Concrete}

Conventional concrete mix was used to simulate medium \& strong pillar and weak and rock, while high strength concrete mix with low water-to cement ratio and chemical additives has been used to simulate strong rock such as sandstone. Table 7-5 showed the proposed strength for medium \& strong coal pillar which are 2,000 and 4,000 psi 
respectively, while the proposed strength for weak rock is 4,000 psi. Two trial batches were prepared to obtain the proportions of concrete mix, water-to-cement (W/C) ratio and the coarse-to-fine aggregate ratio. For each trial batch, three cylindrical concrete specimens of height to diameter ratio $=2$, where their height is 8 in and their diameter is 4 in were cast and cured in water for seven days and then tested to determine their UCS.

Table 7-6 shows the mixture proportions for trial batches \#1 and \#2 to design conventional concrete. The water-to-cement (W/C) ratio is 0.68 and 0.55 for trial batches $\# 1$ and \#2 respectively. The strength of specimens in trial batch \#2 is expected to be higher than that in trial batch \#1 because the water-to-cement ratio is lower in trial batch \#2.

$\underset{\text { Table 7-6 Mixture proportions }}{\left(\mathrm{kg} / \mathrm{m}^{3}\right)}$ for conventional concrete's trial

\begin{tabular}{|c|c|c|}
\hline Trial mix \# & Material & $\begin{array}{c}\text { Amount, } \\
\mathbf{k g} / \mathrm{m}^{3}\end{array}$ \\
\hline \multirow{5}{*}{1} & $\begin{array}{l}\text { Portland cement type } \\
\text { I/II }\end{array}$ & 250 \\
\hline & $\begin{array}{l}\text { Coarse aggregate } \\
\text { (natural gravel) }\end{array}$ & 1,024 \\
\hline & $\begin{array}{c}\text { Fine aggregate } \\
\text { (natural sand) }\end{array}$ & 945 \\
\hline & water & 170 \\
\hline & Water/cement ratio & 0.68 \\
\hline \multirow{5}{*}{2} & $\begin{array}{c}\text { Portland cement type } \\
\text { I/II }\end{array}$ & 355 \\
\hline & $\begin{array}{l}\text { Coarse aggregate } \\
\text { (natural gravel) }\end{array}$ & 1,024 \\
\hline & $\begin{array}{c}\text { Fine aggregate } \\
\text { (natural sand) }\end{array}$ & 870 \\
\hline & water & 198 \\
\hline & Water/cement ratio & 0.55 \\
\hline
\end{tabular}


The most common method of studying the mechanical properties of rocks or concrete is by axial compression of a cylindrical specimen whose length is two to three times the diameter. Figure 7-4 shows the stress-strain curve for six cylindrical concrete specimens in trial batches one and two. The stress strain curves shown in Figure 7-4 are similar to the well-known stress strain curve for rocks under uniaxial compression test. First, there is linear elastic behavior followed by non-linearity close to the ultimate strength. Once the ultimate strength is reached, abrupt failure occurs accompanied by sudden loss of strength. The ultimate strength of specimens in trail batch \#2 is approximately two times the ultimate strength for specimens in trial batch \#1. That's why the cylindrical specimens in trial batch \# 1 are supposed to simulate medium strength pillar, while those specimens in trial batch \# 2 are supposed to simulate strong pillar and weak rock.

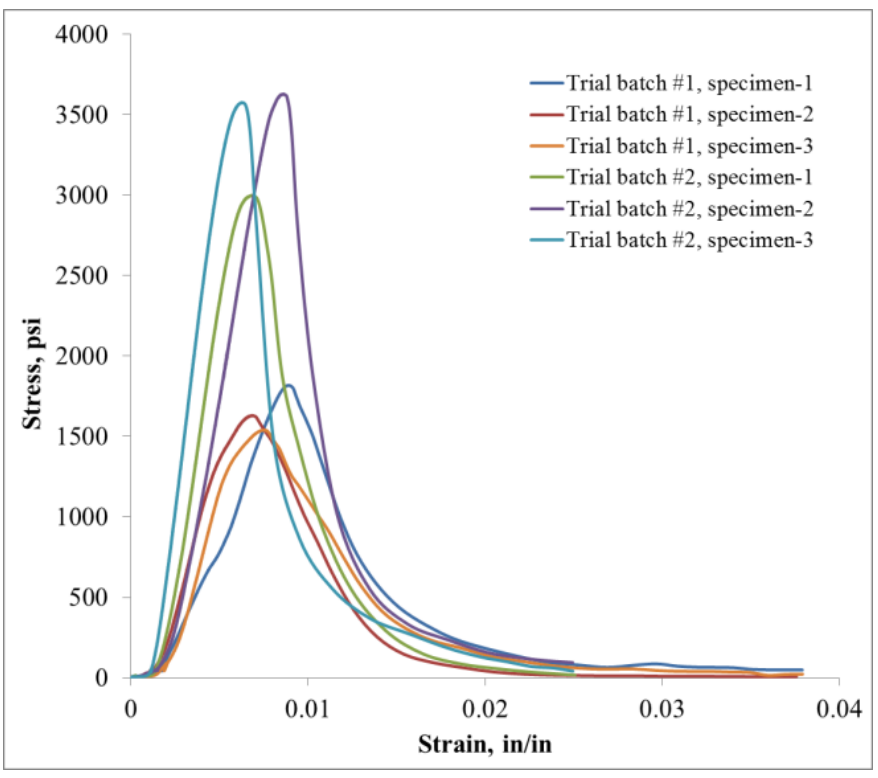

Figure 7-4 Stress-strain curves for trial batches No. 1 and No. 2 to simulate medium and strong pillar 
Table 7-7 shows the UCS and tangent Young's modulus for trial batches shown in Figure 7-5 where these concrete specimens have been tested after 7 days of curing in water. Hence, their strength after 28 days is expected to be 1.3 the values listed in Table 7-7. It is well known that the concrete specimens reach their ultimate strength after 28 days. As shown in Table 7-7, it is obvious that both the UCS and Young's modulus for trial batch \# 2 are higher than those of trial batch \#1.

\section{Table 7-7 UCS and tangent modulus for trial batches of medium and strong pillar}

\begin{tabular}{|c|c|c|c|}
\hline Trial batch & Specimen \# & UCS, psi & E, psi \\
\hline \multirow{3}{*}{$\mathbf{1}$} & 1 & 1,818 & 263,853 \\
\cline { 2 - 4 } & 2 & 1,631 & 452,675 \\
\cline { 2 - 4 } & 3 & 1,539 & 468,866 \\
\hline \multirow{3}{*}{2} & 1 & 2,999 & 806,887 \\
\cline { 2 - 4 } & 2 & 3,630 & 613,310 \\
\cline { 2 - 4 } & 3 & 3,577 & 888,380 \\
\hline
\end{tabular}

The average strength for trial batches \#1 \& \#2 after 7 days of curing are 1,662 and 3,401 psi respectively. The expected strengths for these trial batches after 28 days of curing are 2,216 and 4,535 assuming that the compressive strength after 7 days of curing is 0.75 of the 28 days of curing. So, basically the planned compressive strength of strong pillar is approximately two times that of the medium pillar.

Figure 7-5 shows an example of concrete specimen in batch \#1 before and after failure. The height of the concrete specimen is 8 in while its diameter is 4 in making it a height-to-diameter ratio $=2$. Under unconfined compression, a concrete specimen tends to deform elastically until failure occurs abruptly and this failure is accompanied by 
somewhat irregular longitudinal splitting shown in Figure 7-5b. Splitting occurs in the direction of the major principal stress.

With a moderate amount of confining pressure, longitudinal fracturing is suppressed, and failure occurs along a clearly defined plane of fracture (Jaeger et al., 2007). This plane is typically inclined at an angle less than $45^{\circ}$ from the major principal stress. This type of failure is referred to as shear failure.

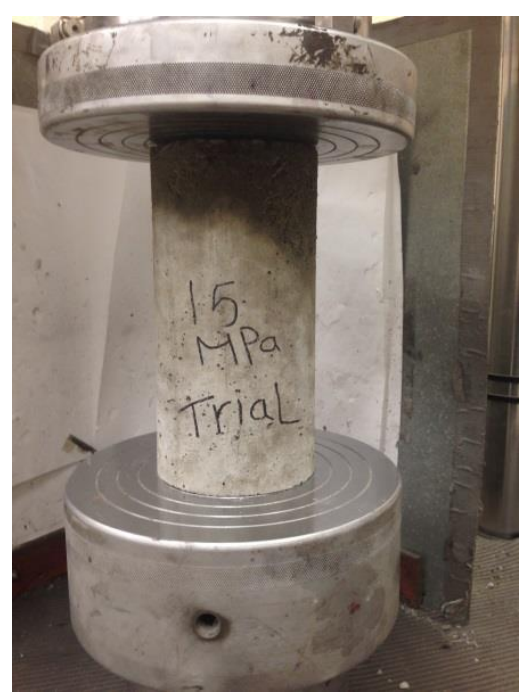

a) A specimen in trial batch \#1 before testing

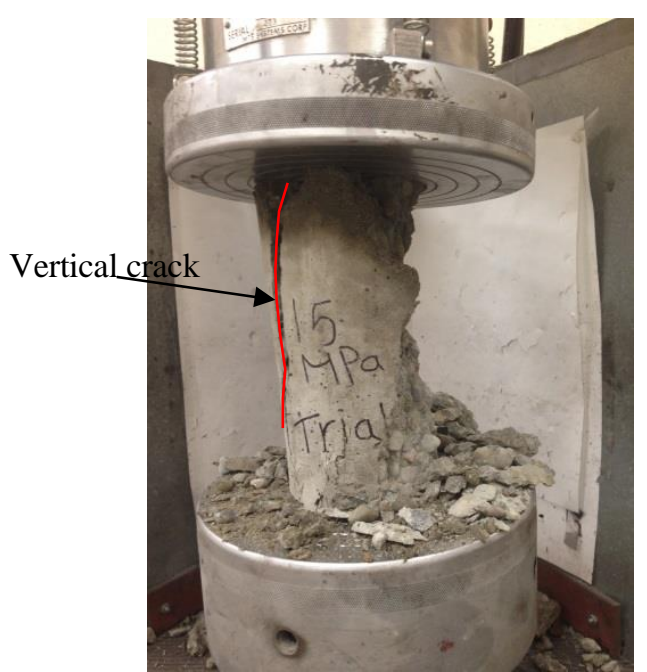

b) A specimen in trial batch \#1 after testing

Figure 7-5 concrete specimen in trial batch\#1 before and after failure

\subsubsection{Trial Batches for High Strength Concrete}

The high strength concrete is planned to simulate strong rock such as sandstone whose ultimate strength is 9,000 psi. Compared to the conventional-strength concrete, the high-strength concretes incorporate higher quantities of cementing materials used in conjunction with multiple types of chemical admixtures (Caldarone, 2009). There are two important points to keep in mind when developing a high-strength concrete and both are related to water-to-binder (W/B) ratio. As the W/B ratio progressively decreases: 
The proportioning principle that were appropriate with the conventionalstrength concrete progressively become less applicable; and

* Some of the constituents that worked well with the conventional concretes become less appropriate.

Developing the high strength concrete capable of satisfying a specific strength is still done largely on a trial and error basis. The true challenge with the high-strength concrete is attaining high mechanical properties while still satisfying durability and constructability (Caldarone, 2009). Contrary to the conventional concrete, chemical additives have been added to increase the strength of high strength concrete. Table 7-8 shows the mix proportions for high strength concrete trial mix. The water-to-binder ratio is 0.35 which is much smaller than conventional concrete mix shown in Table 7-7.

Table 7-8 Trial mix proportion for high strength concrete used to simulate strong rock

\begin{tabular}{|c|c|c|}
\hline Trial mix \# & Material & $\begin{array}{l}\text { Amount, } \\
\mathrm{kg} / \mathrm{m}^{3}\end{array}$ \\
\hline \multirow{8}{*}{1} & $\begin{array}{l}\text { Portland cement } \\
\text { type I/II }\end{array}$ & 500 \\
\hline & Silica Fume & 30 \\
\hline & $\begin{array}{l}\text { Coarse aggregate } \\
\text { (natural gravel) }\end{array}$ & 1100 \\
\hline & $\begin{array}{l}\text { Fine aggregate } \\
\text { (natural sand) }\end{array}$ & 700 \\
\hline & Water & 190 \\
\hline & HRWR & 14 \\
\hline & Retarder & 1.8 \\
\hline & Water/binder ratio & 0.35 \\
\hline
\end{tabular}

Two cylindrical specimens have been prepared using the mix proportion shown in Table 7-8 and cured for seven days and then tested under load control of $320 \mathrm{lb} / \mathrm{sec}$. The 
heights of these specimens are 8 in and their diameters are 4 in. The stress-strain curves for high strength concrete specimens which simulate the strong rock are shown in Figure 7-6. The strength of specimens \#1 \& \#2 are 5,520 and 5,909 psi respectively with an average of 5,715. The expected strength after 28 days of curing is 7,620 psi. Since the proposed UCS to simulate the strong rock is $9,000 \mathrm{psi}$, it is necessary to decrease the water/binder ratio below 0.35 in order to increase the strength.

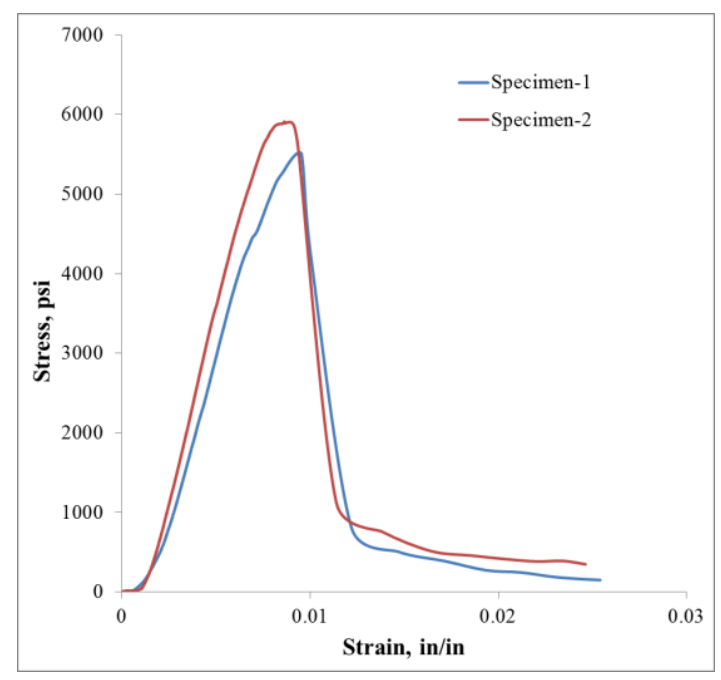

Figure 7-6 Stress Strain curve for high strength concrete trial mix

\subsection{Man-made Concrete Specimens in a Three Layered Approach}

The assembled specimens will be of different unconfined compressive strength to represent eight different combinations of field conditions shown previously in Table 7-1. A plastic mold of 6.0 in diameter and 12 in height is used to cast these man-made concrete specimens. Since every concrete specimen is designed to simulate three different layers, this mold was divided into approximately three equal layers 4.0 in each. The top layer 
represents a roof of specific strength or UCS. The middle layer represents a coal pillar of pre-determined UCS, while the bottom layer represents the floor.

Figure 7-7 shows the procedures for casting the three layers of a concrete specimen. The inner surface of the plastic mold was coated with grease or oily substance to help take the specimen off the mold once it is harden. Since each layer was cast after 24 hours from the next layer, there is a plane of weakness, or interface between each two layers. Therefore, the weakest part in the specimen is expected to be the plane of weakness or interface. It is anticipated that different materials result in different interface friction. However since the three layers are made of the same materials (sand, gravel, cement and water). It has been assumed that the interface friction is the same for all specimens.
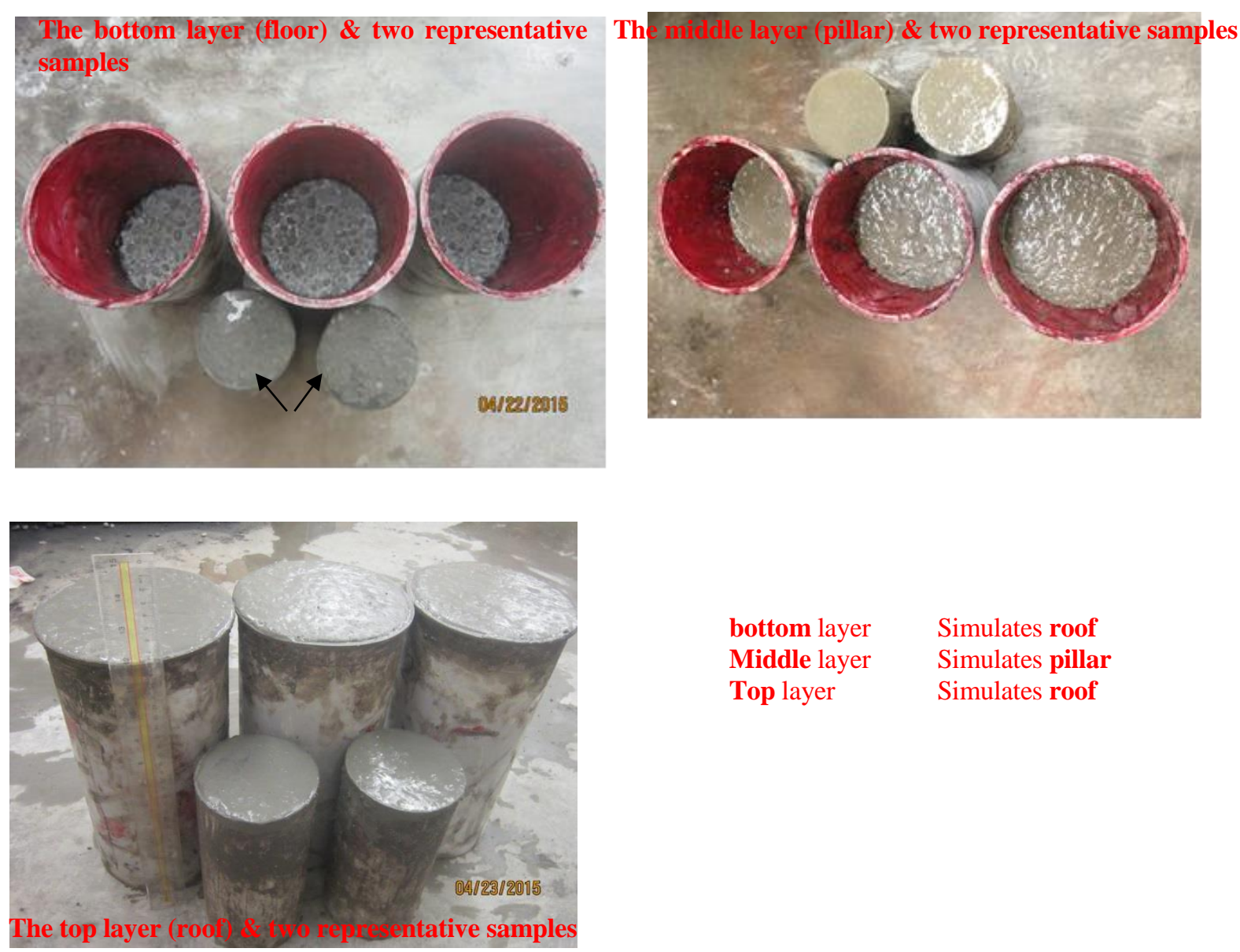
bottom layer
Simulates roof
Middle layer Simulates pillar Top layer Simulates roof

Figure 7-7 procedures for casting the three-layered concrete specimens 
One of the major problems in testing rock specimens is that each specimen is unique. Once it is tested and failed, there is no way to test it again and there is no way to know its strength before running the test. However, the strength of the man-made concrete specimen is well known before running the test because a representative sample, two small specimens, have been taken and tested from the same concrete mix used for each layer. The diameter and the height of these small specimens are 4 in and 8 in respectively. Testing these specimens is conducted according to the ASTM standard.

\subsection{Test Results}

Sandstone, formed by ancient fluvial processes, is the lithology most commonly identified with bumps and rock bursts (Iannacchione and Zelanko, 1995). Rashed and Peng (2015) attempted to determine at what interface friction and width-to-height $(\mathrm{W} / \mathrm{H})$ ratio bumps would occur by investigating the violent failure of coal specimens. They found that the mode of failure of coal specimens can be changed from violent to non-violent or vice versa by changing the $\mathrm{W} / \mathrm{H}$ ratio of coal specimen and the interface friction. Thus according to Rashed and Peng (2015), the failure mechanism of coal specimens is greatly affected by the environment surrounding it which is represented by the interface friction between the pillar and both roof and floor.

To get a complete picture about the effect of geological environment on the failure mechanics of a pillar, the effect of roof and floor strength on the mechanics of failure deserves further consideration. Therefore, investigating the role of roof and floor strength on the mechanics of failure, in addition to the role of interface friction and $\mathrm{W} / \mathrm{H}$ ratio, will help complete the picture of the main causes for bumps. 
Acoustic emission equipment "SoundPro SE/DL instrument" has been used to record the sound pressure level during testing these concrete specimens. The peak sound pressure level (SPL) was used to differentiate between violent and non-violent failures. Moreover, the stress-strain curve for each concrete specimen was studied to see whether or not there is a sudden loss of strength.

\subsubsection{Group 1}

For group \#1, both roof and floor are strong rocks whose strength is designed to be 9,000 psi, while the strength of the medium pillar is designed to be 2,000 psi. Figure 7-8a \& b show the typical stress-strain curves for concrete specimens used to simulate medium pillar and strong rock, respectively. These specimens were taken from the same concrete mix used to cast medium and strong strength layers. Hence, the unconfined compressive strengths for the roof, pillar and floor are known from these representative samples. As shown in Figure 7-8a, the ultimate strengths for three specimens representing medium strength pillar are 2,346, 2,289 and 1,940 psi with an average of 2191 psi.

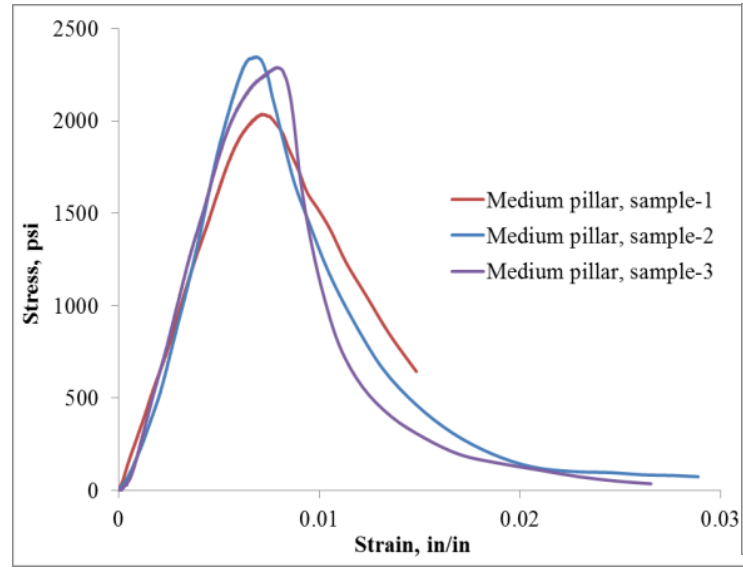

a) Medium pillar

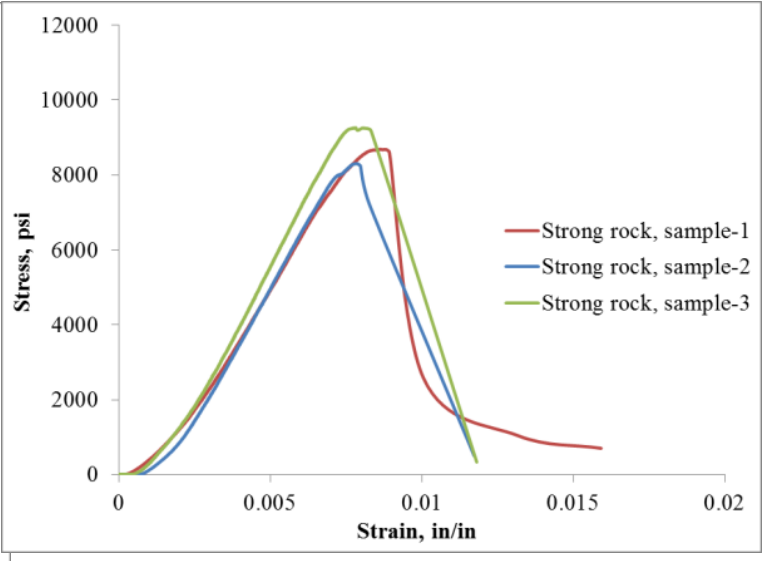

b) Strong rock

Figure 7-8 Stress-strain curves for a) Medium pillar and b) Strong rock 
Figure 7-8b shows the stress-strain curves for three specimens representing strong rock. The ultimate strengths for these specimens are 9,260, 8,686 and 8,303 psi with an average strength of approximately 8750 psi. Please note that the diameter and height of these specimens are 4 in and 8 in with a height-to-diameter ratio $=2$. Hence, the unconfined compressive strength of strong rock and medium strength pillar used in this research is 8,750 and 2,191 psi, respectively, the ratio of UCS for roof-to-pillar or floor-to-pillar is approximately 4 .

Six man-made concrete specimens were casted to simulate group \#1 using a plastic mold of diameter $=6$ in. The total height for each specimen is approximately 10.6 in which is then divided into 3 layers, where the top and the bottom layers simulate the roof and floor, while the middle layer simulates a coal pillar. Hence, the height of each layer is approximately 3.5 in making the height-to-diameter (H/D) ratio $=0.6$ for each layer.

The typical stress-strain curves for three specimens representing group \#1 are shown in Figure 7-9. The failure of concrete specimens simulating group \#1 was smooth in terms of noise pitch. Detailed analysis on acoustic emission data is presented later. The ultimate strength for these specimens are 3,005 \& 3,110 and 3,108 psi respectively with an average strength $=3,074$ psi. 


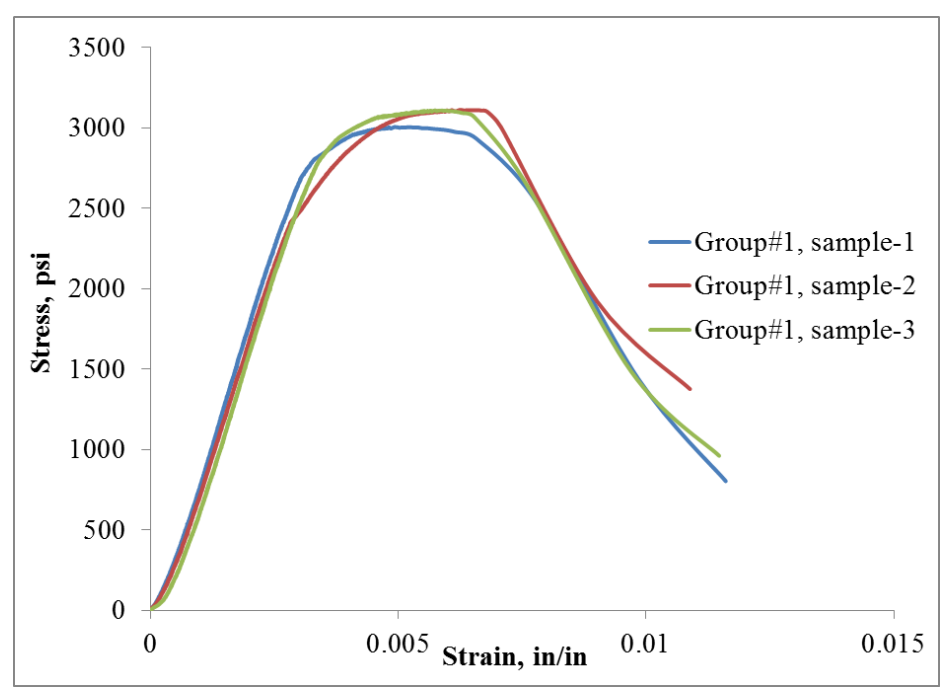

\section{Figure 7-9 Stress-strain curves for group No. 1, strong roof-medium} pillar-strong floor

The average strength for group \#1 (3,074 psi) is very small compared to the proposed strength for a strong roof or floor $(8,750 \mathrm{psi})$, while it is closer to the proposed strength for a medium pillar (2,191 psi). This begs the question: Why does the ultimate strength for group \#1 (3,074 psi) exceed the ultimate strength for the medium strength pillar (2,191 psi)? Since the strength of a medium pillar has been determined from a representative samples of height-to-diameter $(\mathrm{H} / \mathrm{D})$ ratio $=2$ while, the middle layer has $\mathrm{H} / \mathrm{D}$ ratio $=0.6$, and it is well known that the strength of rock specimens increases as its width-to-height ratio increases. Hence, it is anticipated that the strength of the middle layer in group \#1 has to be higher than 2,191 psi. Moreover, the existence of strong roof and floor layers might provide the middle layer in group \#1 with supplemental strength resulting from an interaction between strong and weak layers.

Two dial gauges were attached to each concrete specimen as shown in Figure 7-10 to examine whether or not there is any slip or horizontal shear failure along the roof/pillar interface. The dial gauge attached to the medium strength pillar (middle layer) moved 
slowly a few seconds before failure, while it moves very fast at the moment of failure. Conversely, the dial gauge attached to the strong roof did not experience any movement except when failure occurred probably because the ultimate strength for the roof (strong rock layer) is 4.0 times the ultimate strength of the pillar. So, probably the roof does not experience enough loads to show dilation or lateral expansion.

The movement of the dial gauge attached to the pillar (the middle layer) reflects spalling or bulging and fracture of the pillar. However, the strong roof layer did not experience any spalling or bulging until it fails suddenly. As shown in Figure 7-10, the pillar fails with a combination of vertical and inclined fractures, while the roof layer experienced a splitting failure.
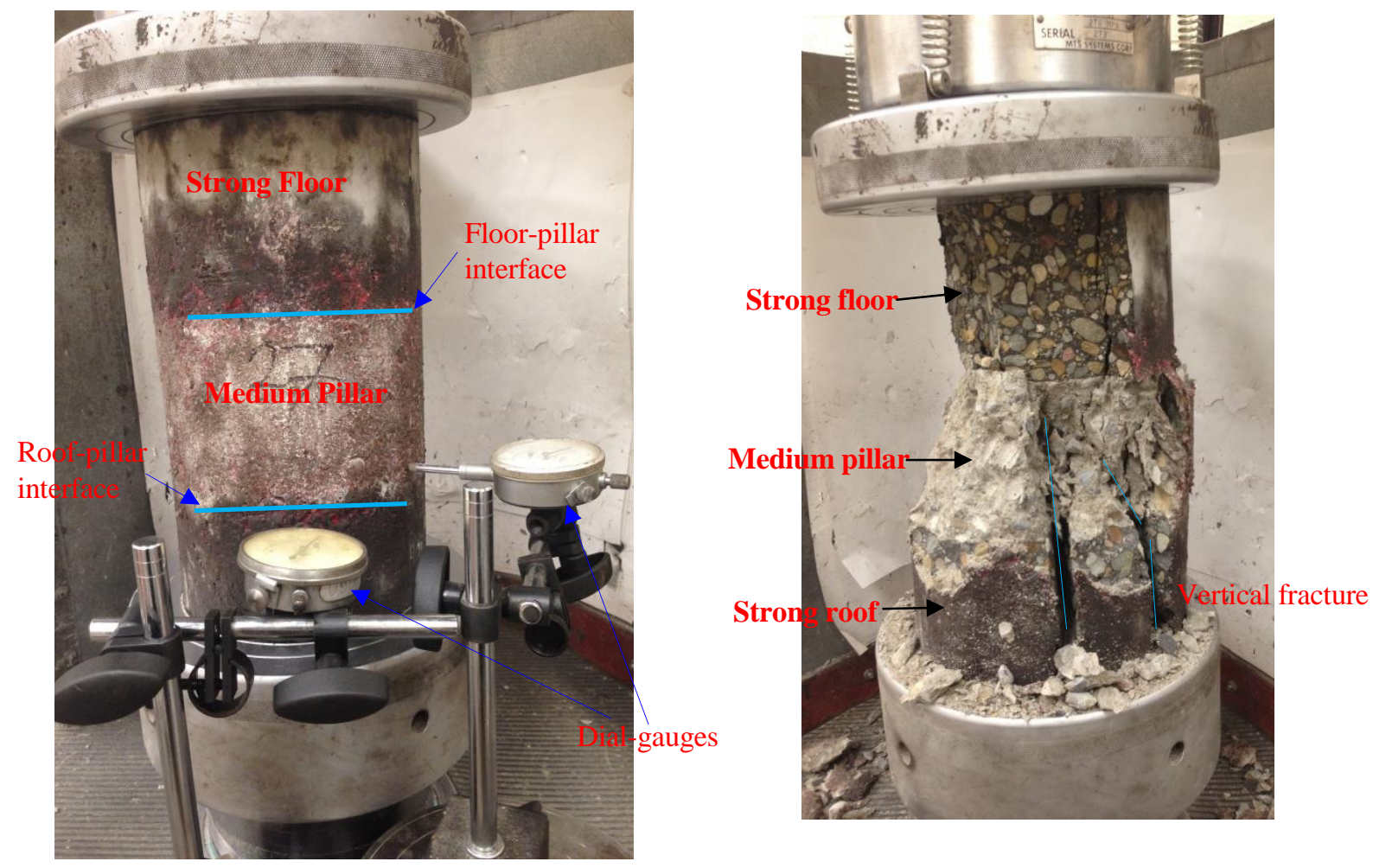

Figure 7-10 Concrete specimen in group No. 1 before and after failure 
A screen shot from a video at the moment of failure for a specimen in group \#1 is shown in Figure 7-11. It indicates that the interface between the roof and the pillar is probably interlocked, because, once the pillar reaches its ultimate strength, it fails abruptly and then the roof layer fails as well with clear vertical tensile cracks resulting in total collapse of the entire system. Please note that the flying debris from the pillar is accompanied by fracture of the strong roof layer.

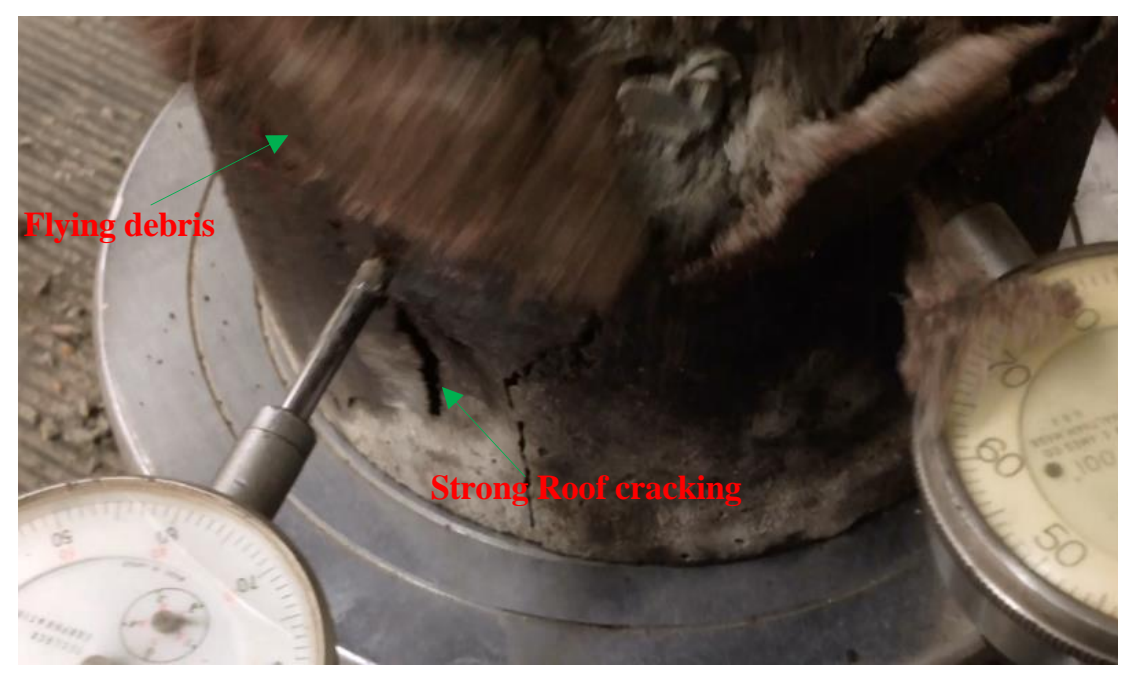

Figure 7-11 A screen shot from video at the moment of failure for specimen in group No. 1

Thus, the test results for group \#1 indicate that failure of the pillar is accompanied by failure of the roof as well. This test results contradict what Brauner (1994) and Rice (1935) documented when coal bumps take place. Brauner observed no fracture in either roof or floor, i.e., the roof and the floor remain stable. Rice (1935) observed two characteristic features of bumps over those pillars which had been crushed where the immediate roof is strong: 1) an open space usually found between the crushed coal and the immediate roof and 2) The immediate roof is neither broken nor thrown down. These observations refer to the hammering effect of the immediate roof. Furthermore, Campoli 
(1987) investigated 5 cases where bumps occurred. He observed that in Mine 2, bumps tend to occur where the thick sandstone directly overlies the coalbed, and no bump generally experienced when various shale lithologies present. Hence, the test results of this research and Rice, Brauner and Campoli field's observations indicate that future research of coal bumps should focus more on the impact load.

Therefore, under static loading conditions of $320 \mathrm{lb} / \mathrm{sec}$ and when a medium strength pillar of ultimate strength 2,191 psi is sandwiched between two strong rock layers of strength $8,750 \mathrm{psi}$, a pillar failure is sudden, accompanied by a non-violent sudden loss of strength.

\subsubsection{Group 2}

For group \#1, the simulated pillar was assumed to have a medium strength $=2,191$ psi, while for group \#2, the pillar was assumed to be strong. Figure 7-12 shows the stressstrain curves for three representative samples of strong pillars. These representative samples were taken from the concrete mix used to cast (strong pillar) in group \#2.

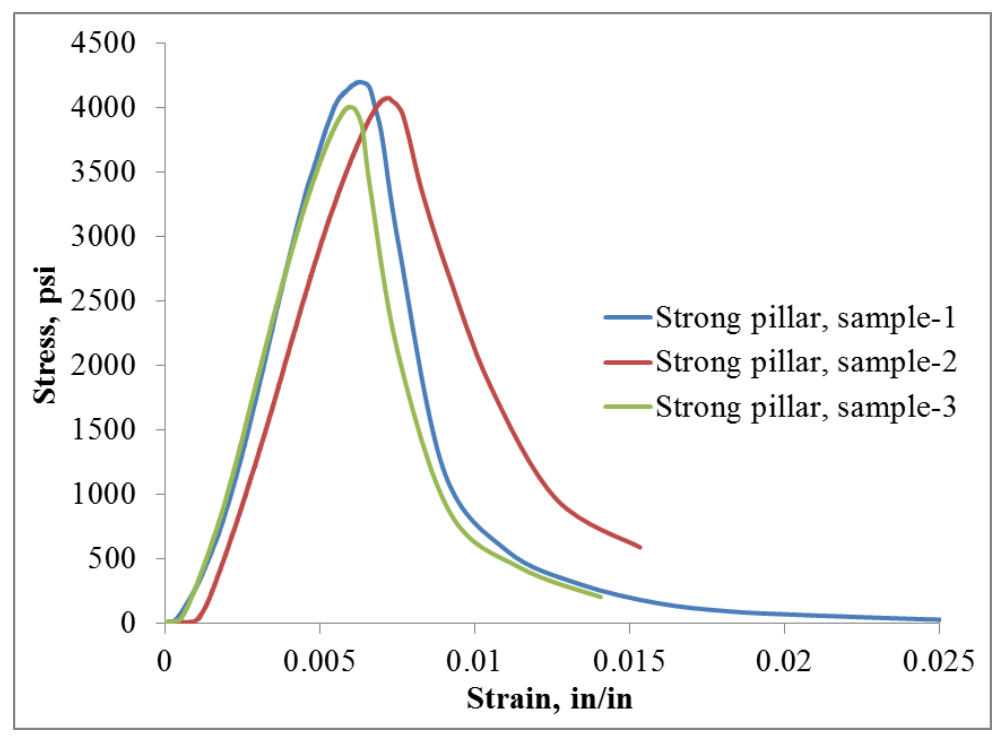

Figure 7-12 Stress-strain curves for strong pillar in group No. 2 
The ultimate strengths for the three specimens shown in Figure 7-12 are 4,200, 4,085 and 4,015 psi with an average UCS of 4,100 psi. In group \#2, the strengths of top and bottom layers are 8,750 psi, while the middle layer represents a strong coal pillar of strength $=4,100$ psi. Hence, the ratio of strength for strong roof-to-strong pillar is approximately 2.1 .

The typical stress-strain curves for three concrete specimens simulating group \#2, strong roof-strong pillar-strong floor, are shown in Figure 7-13. The ultimate strengths for these specimens are 4,423, 4,566 and 5,096 psi. Specimen \#3 attained a higher UCS value probably because it might be more compacted than the other two specimens. The average strength for these three specimens is 4,695 psi which is much less than the ultimate strength of strong roof and floor layers $(8,750 \mathrm{psi})$. However, the average strength for the specimens in group \#2 (4,695 psi) is slightly higher than the average strength of a strong pillar $(4,100)$. Probably because of the shape effect, as explained earlier in group \#1.

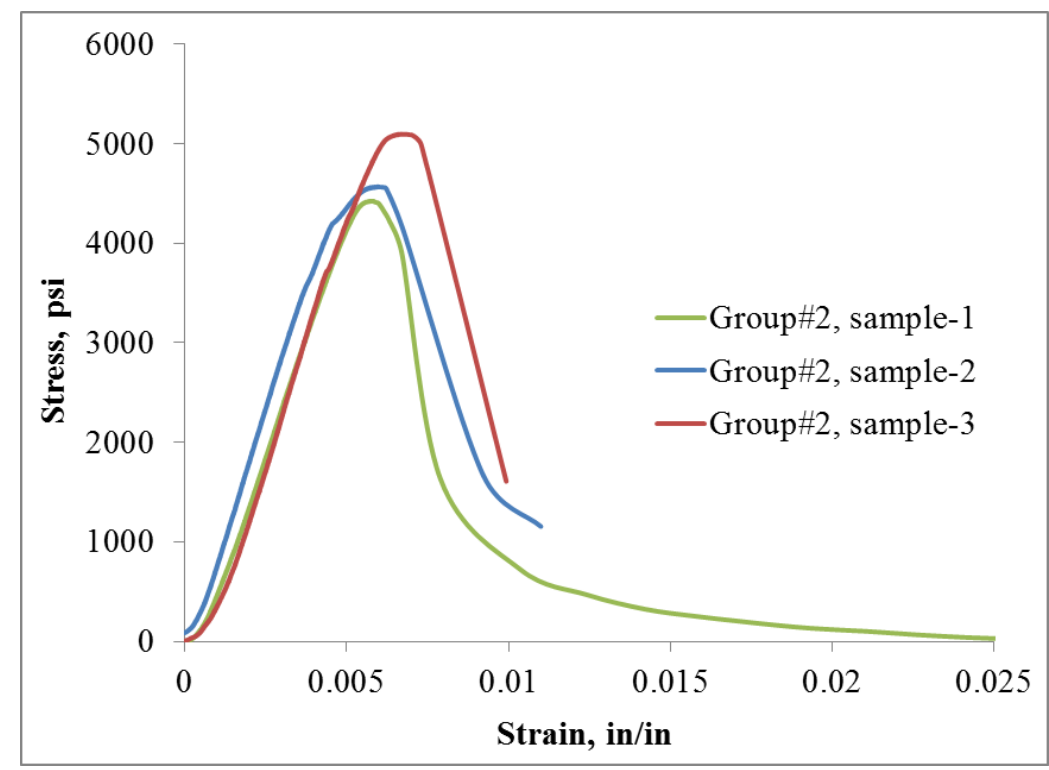

Figure 7-13 Typical stress-strain curves for specimens in group-2, strong roof-strong pillar-strong floor 
Although the failure of concrete specimens simulating group \#2 was abrupt, where the failure occurred once the peak strength was reached, it was not violent in terms of noise. More about noise results will be shown later.

Figure 7-14 shows an example of concrete specimen in group \#2, strong roof-strong pillar-strong floor, before and after failure. The pillar failed with inclined fractures close to $45^{\circ}$ angle which suggests more confinement or constraint, while the strong roof fails with vertical fractures suggesting a tensile failure. It was noticed that both the dial gauges attached to the strong pillar and strong roof experienced movement before specimen failure. Such movement suggests considerable spalling or horizontal movement before failure. However, the movement of the dial gauge attached to the strong pillar was faster than the one attached to the strong roof. The movement of the dial gauge was faster at the moment of failure.
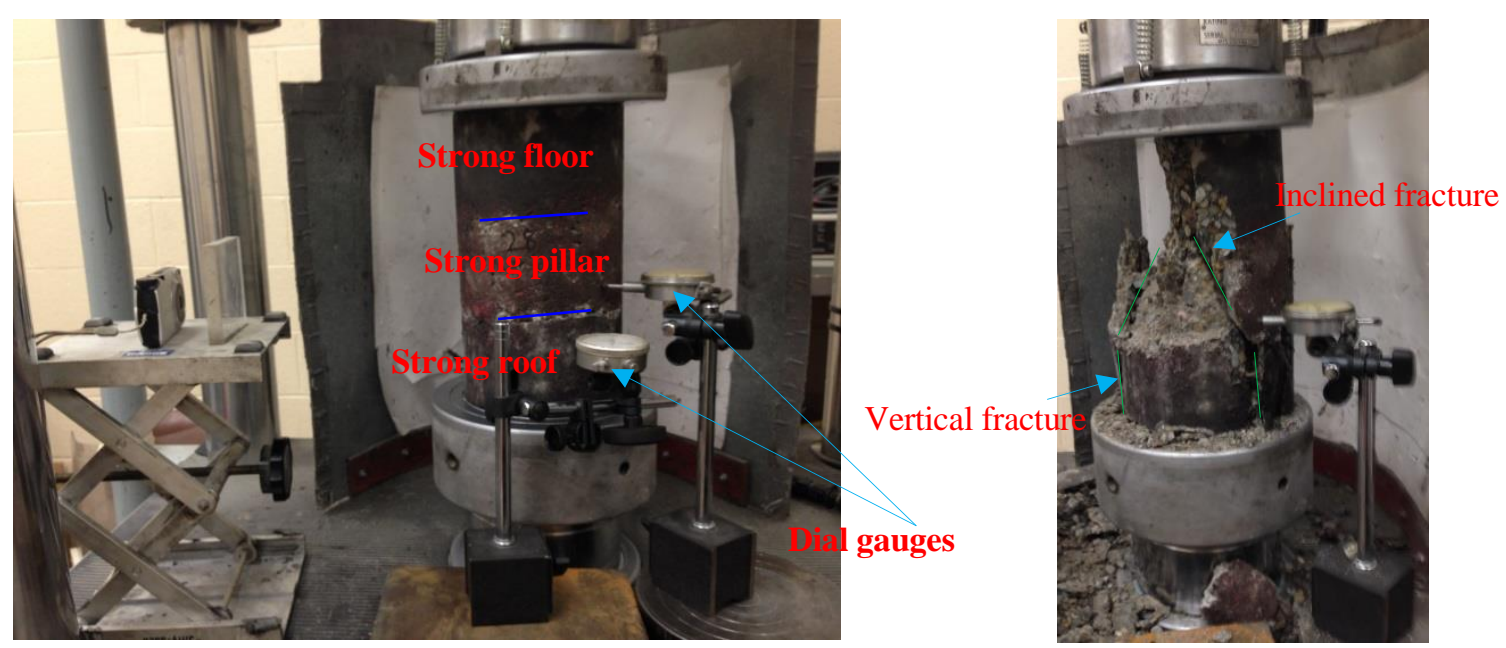

Figure 7-14 A concrete specimen in group No. 2 before and after failure 


\subsubsection{Comparing Acoustic Emission for Groups No. 1 \& No. 2}

SoundPro SE/DL instrument was used to record the sound pressure level (SPL) during testing concrete specimens for groups \#1 \& \#2. The peak sound pressure can be used as a guide to assess the mode of failure whether it is violent or not. Basically, violent failure is expected to have high noise pitch, while non-violent or smooth failure should be accompanied by low noise pitch. Prassetyo (2010) used the peak sound pressure level (SPL) to assess the violent failure of coal specimens. He proposed that the mode of failure is violent when the peak SPL is greater than $124 \mathrm{~dB}$.

Figure 7-15 shows the SPL levels for six specimens representing group \# 1 (strong roof-medium pillar-strong floor) and six specimens for group \# 2 (strong roof-strong pillarstrong floor). For group \#2, the average SPL is approximately $89 \mathrm{~dB}$, while for group \#1 the average SPL is approximately $80 \mathrm{~dB}$. Hence, the potential for violent failure increases with increasing the strength of the pillar, because the SPL is higher for group-2. However, in both cases, the SPL is far from the $124 \mathrm{~dB}$ recommended by Prassetyo to distinguish between violent and non-violent failures. 


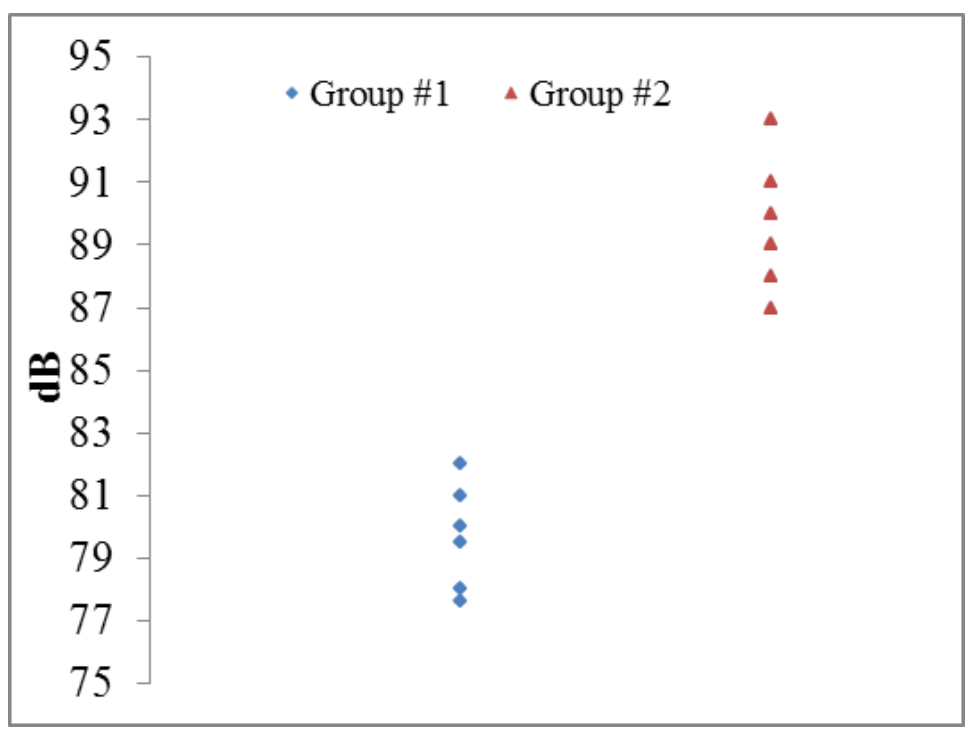

Figure 7-15 Peak SPL for specimens in groups No. 1 and No. 2

Therefore, under static loading conditions, squeezing the medium strong pillar of ultimate strength $=2,191 \mathrm{psi}$ or strong pillar of ultimate strength $=4,100$ psi between two strong rock layers of strength $=8,750 \mathrm{psi}$, specimen failure would be sudden however not violent. A question followed: what if the strength of pillar remains the same, while the strength of strong roof and floor is much higher than the 8,750 psi? Does the mode of failure change or not? It is expected that even if the strength of roof and floor is higher than the proposed value, the mode of failure will not change because what was found from the test results of groups \#1 and \#2 specimens is that once the ultimate strength of the pillar (either 2,191 or $4,100 \mathrm{psi}$ ) is reached, it fails suddenly. It does not matter how high the strength of roof and floor. Furthermore, the roof would experience tensile failure once the pillar fails.

\subsubsection{Groups No. 3 and No. 4}

Group \#3 simulates a weak roof-medium pillar-strong floor, while group \#4 simulates a weak roof-strong pillar-strong floor. Strong and weak rock have an UCS = 
8,750 and 4,100 psi, respectively. While the medium and strong pillars have an UCS = 2,191 psi and 4,100 psi, respectively.

Figure 7-16 shows the typical stress-strain curves for two specimens in group \#3 and two specimens in group \#4. The ultimate strengths for the specimens in group \#3 are 2,639 and 2,495 psi while the ultimate strengths for the specimens in group \#4 are 4,398 and 4,129 psi. The stiffness of specimens in group \# 4 is higher than that of group \#3, since the slope of the stress-strain curve is higher for specimens in group \#4.

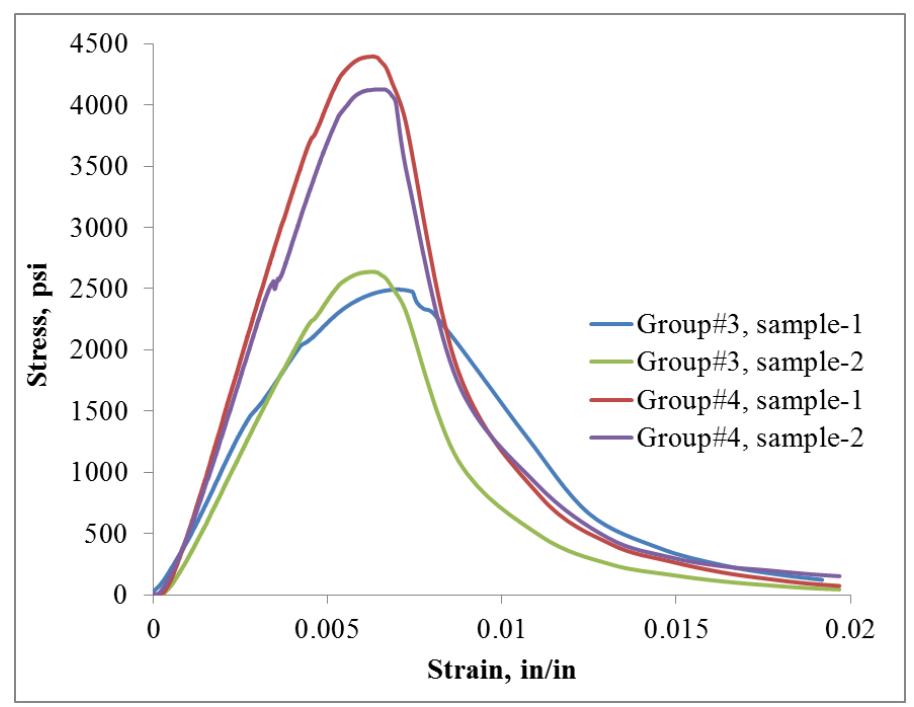

Figure 7-16 Stress-strain curves for specimens in groups No. 3 and No. 4

For group \#3, the weakest link or layer in that system is the middle layer (pillar), while the weakest link in group \#4 is either the pillar or the roof, because the strength of weak rock equals the strength of strong pillar. As shown in Figure 7-16, the ultimate strength for specimens in groups \#3 and \#4 is closer to the weakest layer. It does not matter how strong one or two layers as long as one layer is weak, failure occurs in that weak layer and once it fails the whole system fails. This is a common mode of failure for specimens subjected to quasi-static loading. 
The failure of specimens in groups \# 3 and \# 4 was sudden however non-violent in terms of noise pitch. A comparison between the peak SPL for groups \# 3 and \#4 is shown in Figure 7-17. The average peak SPL for group \# 4 is 83.5, while for group \# 3, it is $81 \mathrm{~dB}$. Hence, for the same geological conditions, as the strength of pillar increases, the potential for violent failure increases. The Peak SPL for groups \# 3 and \# 4 is less than $124 \mathrm{~dB}$ proposed by Prassetyo (2010) to differentiate between violent and non-violent failures.

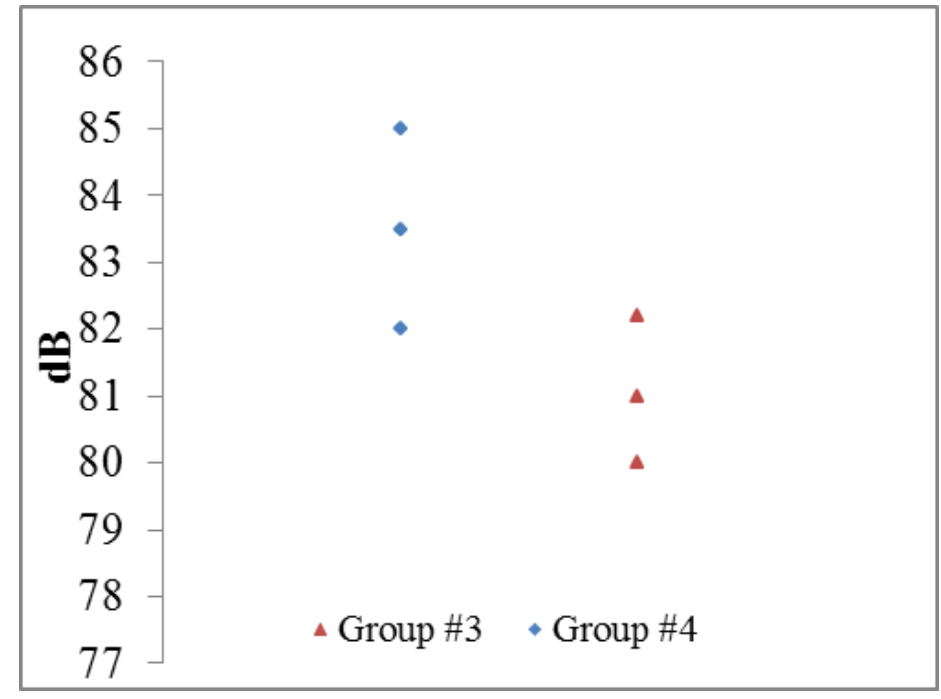

Figure 7-17 Peak SPL for groups No. 3 and No. 4

\subsubsection{Groups No. 5 and No. 6}

Group \#5 is represented by a strong roof-strong pillar-weak floor, while group \#6 is represented by a strong roof-medium pillar-weak floor. The bump prone coal seams are always overlain by a strong and stiff stratum or strata such as sandstone or limestone, or massive shale (Peng, 2008).

Under static loading condition of $320 \mathrm{lb} / \mathrm{sec}$, the mode of failure and the peak SPL for groups \#5 and \#6 have been studied to see whether or not a violent failure would happen. The typical stress-strain curves for two specimens simulating groups \#5 and \#6 are shown in Figure 7-18. The ultimate strengths for specimens in group \#5 are 4,872 and 
4,549 psi, which is very small compared to the strength of strong roof. However, it is closer to the strength of strong pillar or weak floor. For group \#6, the ultimate strengths for the specimens are 3,518 and 3,085 psi which is closer to the strength of the weak layer. Hence, under static loading conditions, the weakest layer determines the strength of the entire system, no matter how high the strengths of other two layers are.

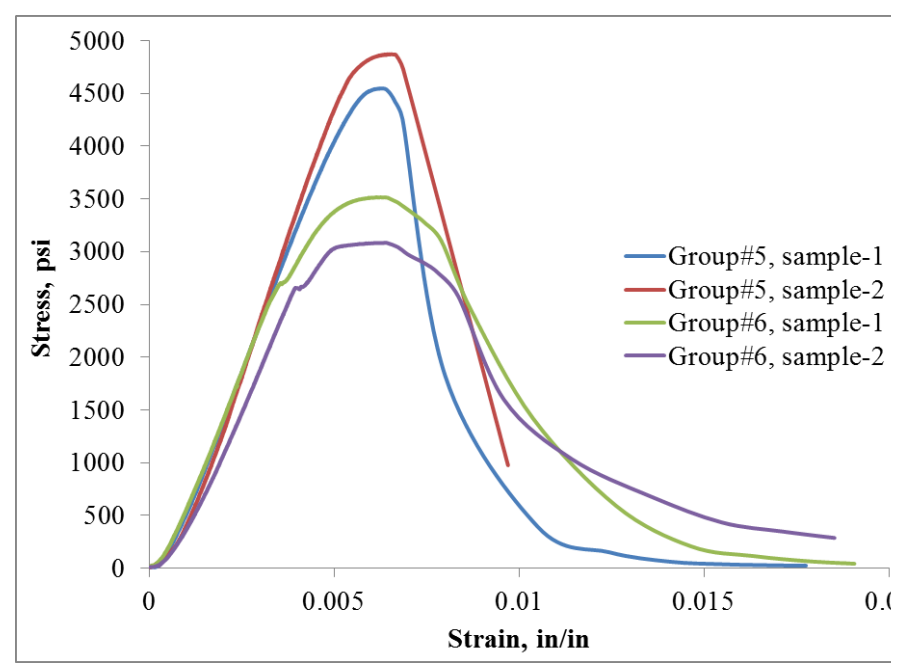

Figure 7-18 Typical stress strain curves for specimens in groups No. 5 and No. 6

A concrete specimen before and after failure simulating geological condition \#5 is shown in Figure 7-19, strong roof-strong pillar-weak floor. The strength of strong roof is approximately two times the strength of pillar, and the strength of pillar equals the strength of weak floor. The roof has only one big crack, while the pillar and the floor have many cracks, 

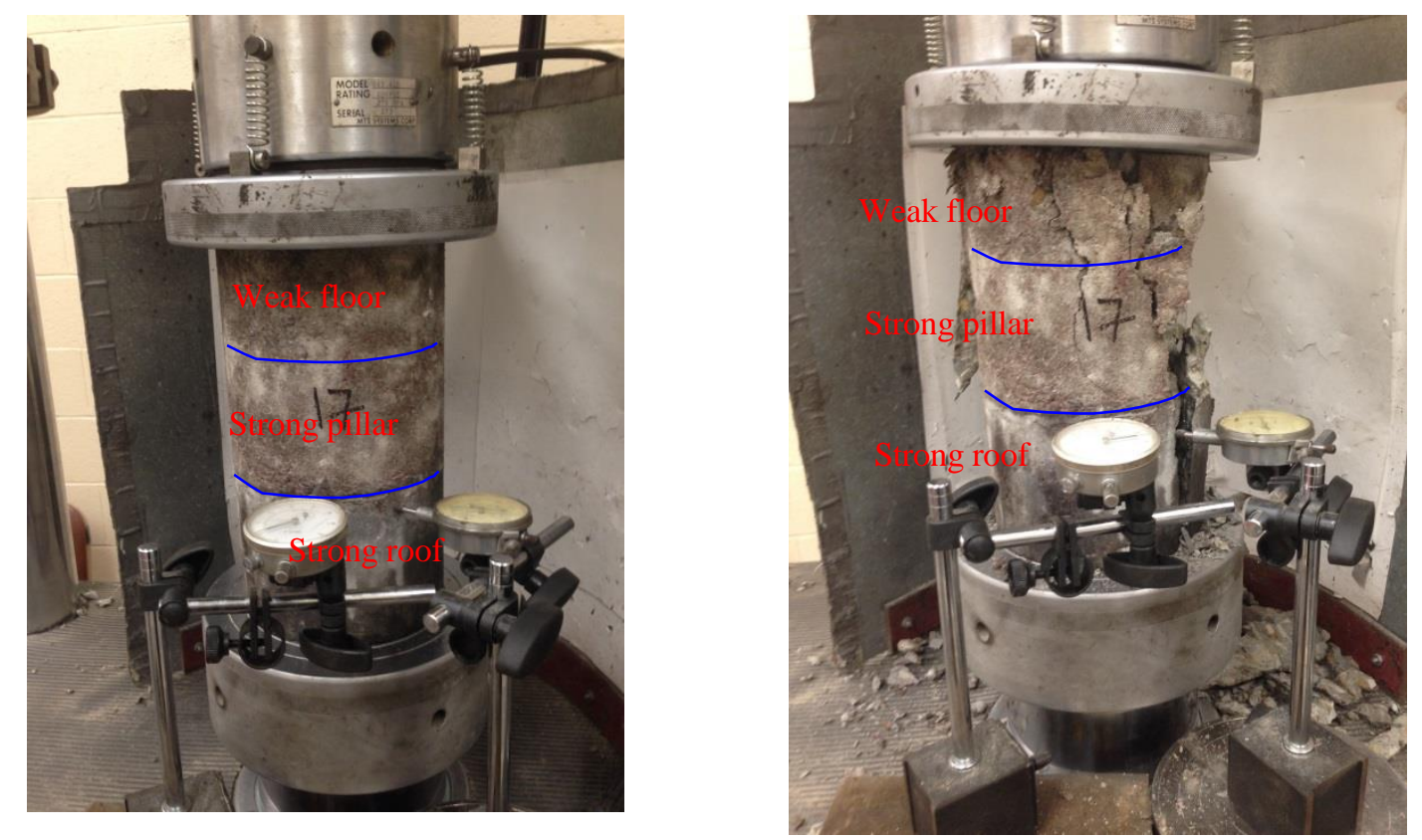

Figure 7-19 A concrete specimen in group No. 5 before and after failure

Figure 7-20 shows a concrete specimen before and after failure for group \# 6 . The pillar has the weakest strength $(2,191 \mathrm{psi})$, while the roof has the highest strength $(8,750$ psi). The strength of the floor is 4,100 psi. The roof did not experience any damage, only one fracture split the roof into two parts, while the pillar and the floor are highly damaged and have many cracks and fractures. Basically, it is much easier for the cracks to propagate in the pillar and the floor rather than the roof which is characterized as strong rock. 

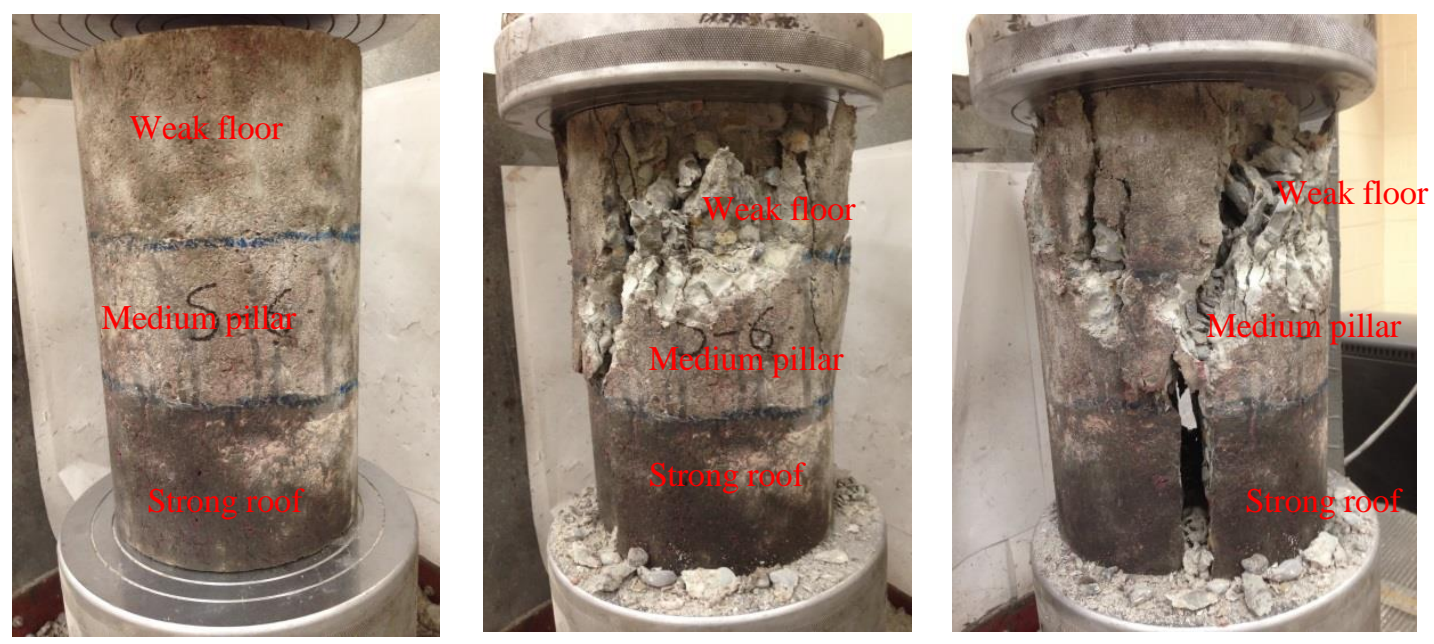

Figure 7-20 A concrete specimen in group No. 6 before and after failure

Although the failure of concrete specimens simulating field conditions \#5 and \#6 was abrupt, it was not violent. As shown in Figure 7-21, the average peak SPL for the specimens simulating field condition \# 5 is 84 , while it is approximately 77 for field condition \#6 which are very small compared to the threshold $124 \mathrm{~dB}$ proposed by Prassetyo (2010).

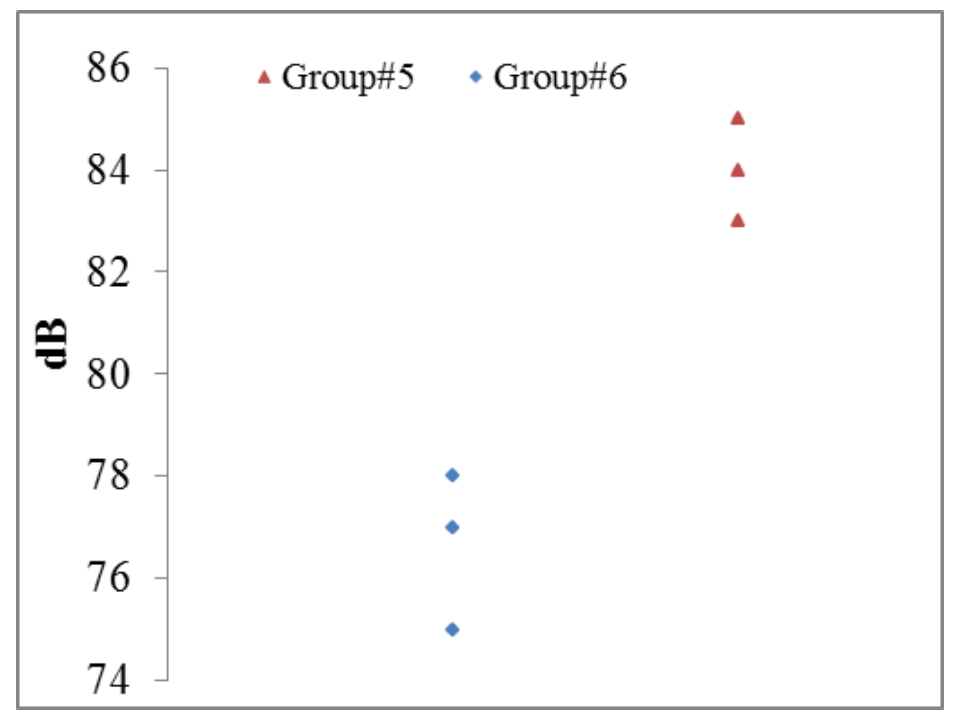

Figure 7-21 Peak SPL for groups No. 5 and No. 6 


\subsubsection{Groups No. 7 and No. 8}

Group \#7 is represented by a weak roof-medium pillar-weak floor, while group \#8 is represented by a weak roof-strong pillar-weak floor. The typical stress-strain curves for groups \#7 and \#8 are shown in Figure 7-22. The stress-strain curves are very similar to the other field conditions discussed previously, where a sudden loss of strength occurs once the ultimate strength is reached. However, the failure for group \#7 and \#8 was very smooth where the noise level was low. For group \#8, the three layers have the same strength, while for group \#7 the middle layer (the pillar) is weaker than the roof and the floor. The ultimate strength is higher for group \#8 than that for group \#7 because the pillar is weaker than the roof and the floor for group \#7.

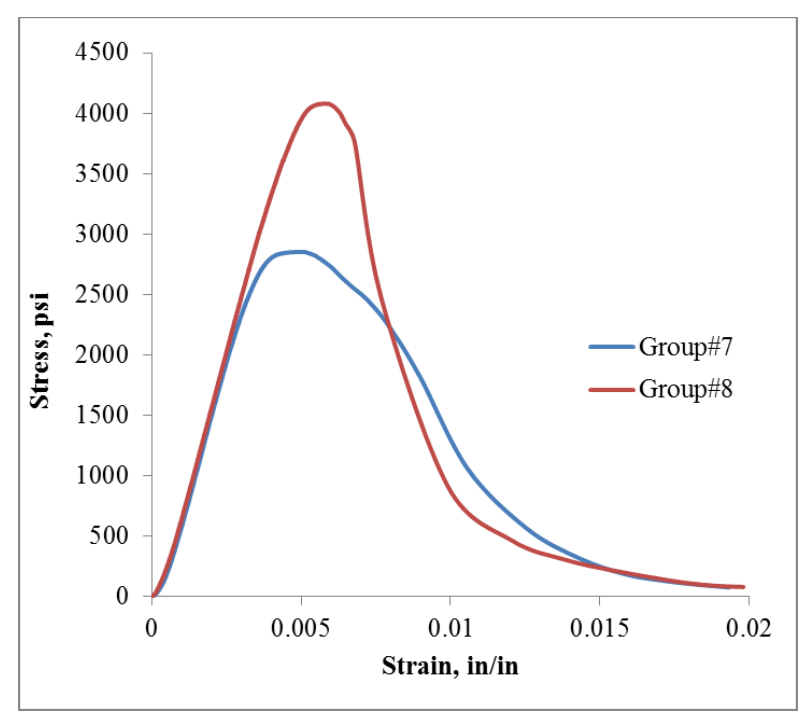

Figure 7-22 Typical stress-strain curves for specimens in groups No. 7 and No. 8

Figure 7-23 shows the peak SPL for groups \#7 and \#8. The average SPL's are 74 and $76 \mathrm{~dB}$ for groups \#7 and \# 8, respectively. By comparing the peak SPL for field conditions \#7 and \#8 with other cases, it was found that the SPL is less when both the roof 
and floor are weak rocks. Therefore, under static loading conditions the potential for violent failure increases with the existence of strong roof and floor. However, even when both the roof and floor are strong rocks the noise level produced during specimen failures was not high enough to claim that a violent failure occurs just because of strong roof and floor.

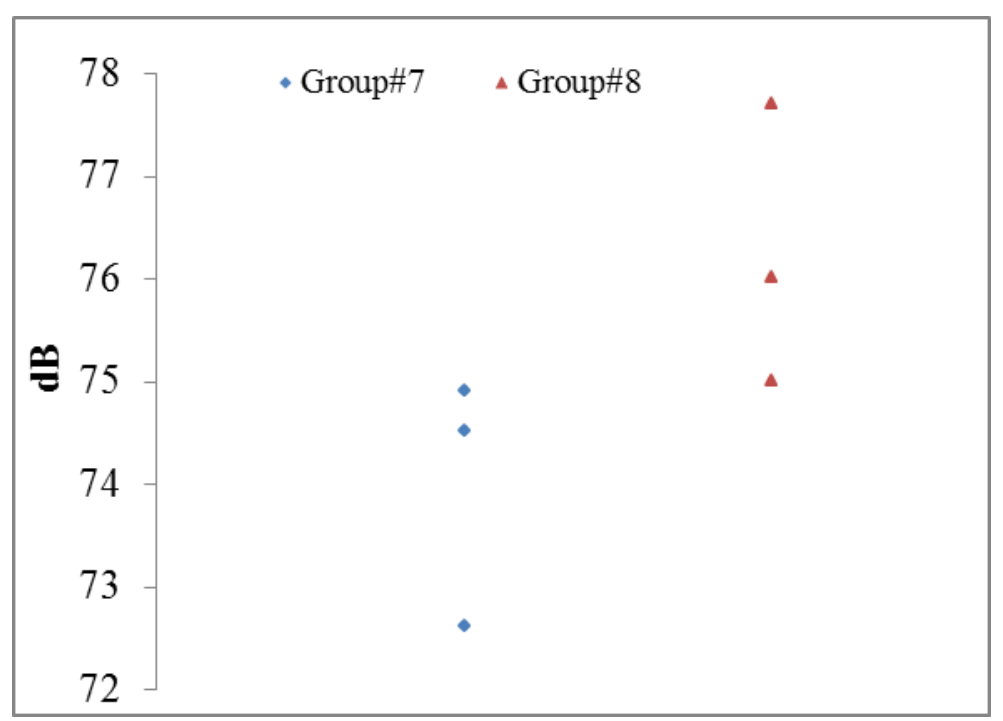

Figure 7-23 Peak SPL for groups No. 7 and No. 8

Figure 7-24 shows the concrete specimens before and after failure for groups \#7 and \#8. The pillar for group \#7 is damaged more than that for group \#8.
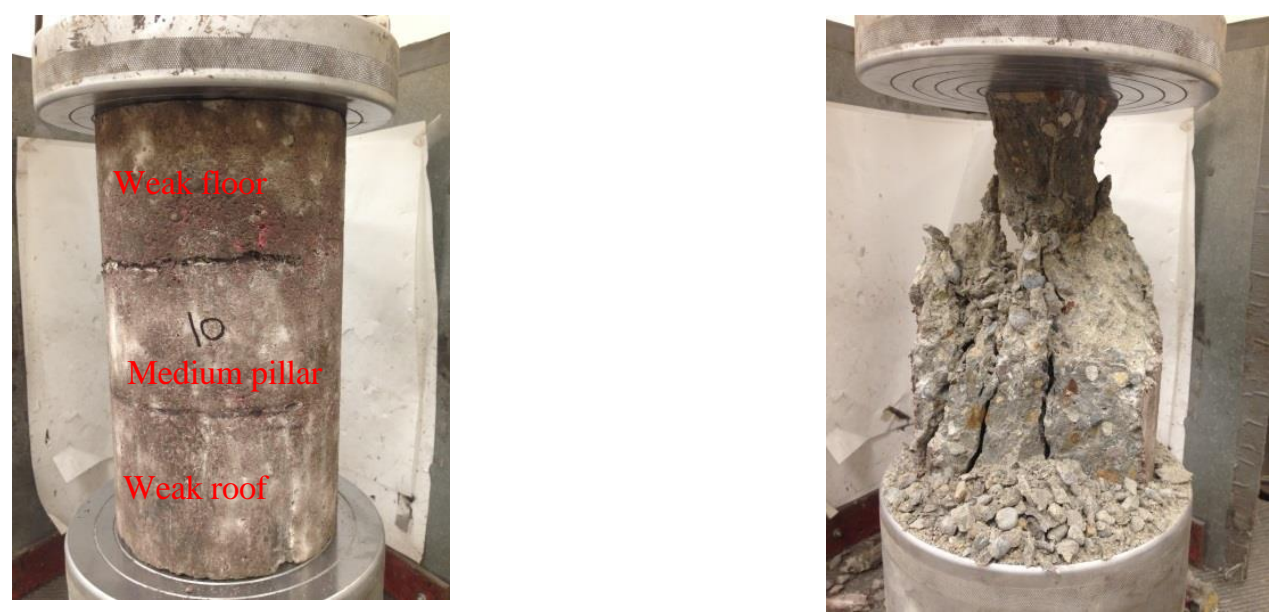

a) Group No.7 

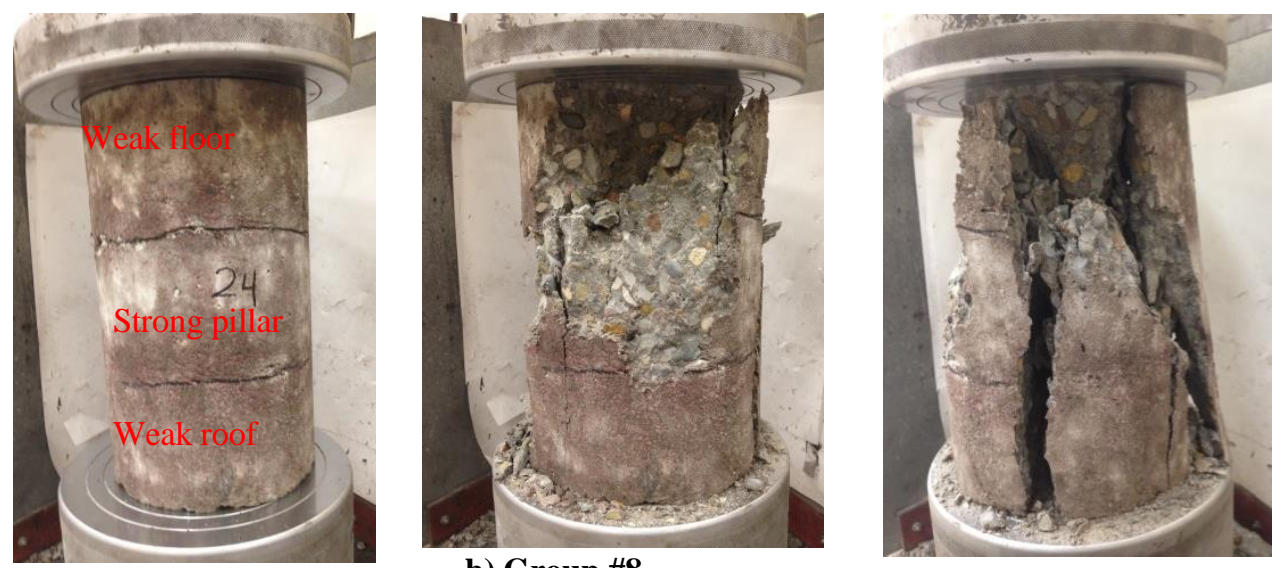

Figure 7-24 Concrete specimens before and after failure for a) group No. 7 and b) group No. 8

Generally for the eight field conditions studied in this research, it was found that under static or quasi-static loading conditions, the failure of man-made concrete specimens was sudden, however non-violent, where the noise level was not high enough to say it was a bump. Furthermore, the noise level tended to be slightly higher for specimens whose roof and/or floor were strong rocks. The existence of strong roof and floor did not cause a violent failure.

It is more likely that the impact of a strong roof and floor on the potential for violent failure would be more obvious when these tests were conducted under dynamic loading conditions rather than the static loading condition adopted in this research.

\subsection{Summary for Chapter Seven}

For the man-made concrete specimens of groups \#1 and \#2, when a medium pillar of $\mathrm{UCS}=2,191$ or strong pillar of $\mathrm{UCS}=4,100$ psi is sandwiched between strong the roof and floor of strength $=8,750 \mathrm{psi}$, the failure of pillar was found to be sudden, where there is instantaneous loss of strength once the ultimate strength is reached, however it is nonviolent in terms of noise level. The average Peak SPL is approximately $94 \mathrm{~dB}$ when the 
pillar is strong, while is it approximately 80 when it is medium strong pillar. The peak SPLs are far from the $124 \mathrm{~dB}$ proposed by Prassetyo (2011) to differentiate between violent and non-violent failure. Furthermore, it was found that once the pillar fails, the roof fails as well. In the actual field cases where bumps occurred, the roofs were still intact and stable and there was a gap between the roof and the crushed pillar. For groups \#3 to \#8 where strong rock exists only in either roof or floor, no matter what pillar strength is, the failure was not violent however it was sudden. The lowest peak SPL occurred when both the roof and floor are weak rocks, and the highest Peak SPL occurred when both the roof and floor are strong rocks.

According to the experimental work in chapter seven, to say a strong roof and floor are enough for a burst to occur would be incorrect under static loading conditions. However, other factors should be taken into considerations as well such as, the interface friction and $\mathrm{W} / \mathrm{H}$ ratio. 


\section{CHAPTER 8: SUMMARY AND CONCLUSIONS}

Violent failures of coal pillar (s) which is known as coal mine bump (s) in practice, have long been a major hazard for U.S. Coal Mines. Because of the catastrophic nature of these sudden failures, understanding the cause of failure and developing mitigation techniques for controlling such failures have been the main objective for many studies. The

main objective of this research is to understand the mechanics of coal bumps in a laboratory setting and then mitigate them.

Although most of the work done in this dissertation was laboratory work conducted on coal specimens, the main findings and conclusions can be extended to coal pillars as long as coal specimens and coal pillars have the same material (coal), aspect ratio $(\mathrm{W} / \mathrm{H}$ ratio), constraint (interface friction), and the same geological cycle (the history of tectonic stresses).

\subsection{Summary for this Research}

This research can be summarized in the following questions:

1. The role of the mechanical properties of the coal play role in coal mine bumps. A comparison has been conducted between two kinds of coal one from bump prone-mine, the Sunnyside coal seam, (the Utah coal) and the other one non bump-prone mine, the Sewickley seam, (the WV coal). The following points summarizes the role of the mechanical properties: 
a. The UCS of the Sunnyside coalseam, Utah coal is 1.2 times greater than the UCS for the Sewickley coalseam, WV coal. The $20 \%$ difference in UCS is not sufficient to determine bump-prone or non-bump-prone.

b. The tri-axial strength for the Sunnyside coalseam, Utah coal is 1.25 times the tri-axial strength of the Sewickley coalseam, WV coal. This is still not significantly high enough to be confident that the strength of coal plays a significant role in bump.

c. Since the Young's modulus is almost the same for both the Sunnyside coalseam, Utah coal and the Sewickley coalseam, WV coal, it is unlikely to play a significant role in the coal bumps.

d. The burst index is a good method to distinguish between bump-prone and non-bump-prone coal (the Utah and the WV coals). The Utah coal (Sunnyside seam) is classified as severely susceptible to bump because it has the ability to store more energy, while dissipating little energy. The average burst index for Utah coal is approximately 3 times that for the WV coal.

e. Since the mechanical properties of the coal (UCS, Young's modulus and shear strength) do not play a significant role in bump. That means the local variation of the geological conditions and the geometry are the controlling factor in bump.

2. The role of interface friction and the width-to-height ratio were investigated on the potential for violent failure. The following points summarize how the mode 
of failure of coal specimens changes from a violent to non-violent behavior, depending on the variation of the interface friction and the width-to-height ratio.

a. The mode of failure for uniaxial loaded coal specimen or coal pillar is a combination of both shear slip along the interface and compressive failure in the coal. The shear failure along the interface triggers the compressive failure in coal. Basically, controlling the shear failure along the interface is not feasible. However, the compressive failure of coal specimen be controlled, because it depends on its width-to-height ratio.

b. With a proper combination of interface friction and $\mathrm{W} / \mathrm{H}$ ratio of a coal specimen, the mode of failure would be violent or non violent. Coal specimens were divided into five different groups according to their interface friction and $\mathrm{W} / \mathrm{H}$ ratio.

c. Up to a certain level, the degree of violence in terms of noise and ejections increases with increasing the $\mathrm{W} / \mathrm{H}$ ratio and the interface friction.

d. When the interface friction between coal is 0.1 and the $\mathrm{W} / \mathrm{H}$ ratio ranges from 1 to 3.8 , the expected mode of failure is sudden brittle failure, but not too violent in terms of noise and ejection. So even a very low interface friction does not prevent the sudden failure.

e. When the interface of friction is 0.1 and the $\mathrm{W} / \mathrm{H}$ ratio ranges from 4.7 to 6.5 , the mode of failure changes from brittle to ductile failure with clear plastic strain and permanent deformation. However the mode of failure is strain softening. 
f. When the interface friction is 0.1 and the $\mathrm{W} / \mathrm{H}$ ratio ranges from 6.8 to 10, failure is very stable with strain hardening. Coal specimens expand and are squeezed with very low noise and ejection. This combination of interface friction causes stable slip along the interface and eventually non-violent failure.

g. When the interface friction is 0.25 and $\mathrm{W} / \mathrm{H}$ ratio is less than 7.7 , failure is sudden and violent. The intensity of violence increases with increasing UCS. $\mathrm{W} / \mathrm{H}$ ratio=7.7 is the threshold limit between violent and nonviolent failures of coal specimens. Stable slip along the interface occurs when $\mathrm{W} / \mathrm{H}$ ratio $=7.7$ and interface friction $=0.25$.

h. Under static or quasi-static loading conditions, every interface friction is associated with a specific $\mathrm{W} / \mathrm{H}$ ratio at which stable slip along the interface and eventually non-violent failure would occur. In this case if bump occurs, it belongs more likely to the shock bumps as defined by Rice (1935).

i. Therefore, based on this research bumps would occur only when the interface friction is 0.25 and $\mathrm{W} / \mathrm{H}$ ratio is less than 7.7 , any other combination of $\mathrm{W} / \mathrm{H}$ ratio and interface friction higher than 0.25 remains to be determined.

j. It was proved experimentally by testing 13 coal specimens having different $\mathrm{W} / \mathrm{H}$ ratios and interface frictions that once the specimen failed, the core zone is not elastic any more. Because it was found that the stiffness of the core zone is very small as compared to the stiffness of 
intact coal specimen, which gives an indication that the core zone is damaged to some extent.

3. Exploring two of the most common proposed bump mechanisms. Although two of the most common bump mechanisms were proposed many years ago, no work has been done to explore them. So the Finite Element Models have been generated to investigate the validity of sudden loss of constraint and sudden impact load as mechanisms for coal bumps. The main point from this investigation is that sudden kinetic and elastic strain energies released when a coal specimen experienced sudden loss of constraint or sudden impact load. It is believed that these two kinds of energies are required for the occurrence of bump, because the elastic energy is needed for the crack initiation and propagation (break the pillar), while the kinetic energy is required to eject the broken debris away from the pillar into the entry and /or the cross-cuts. Basically, future research should focus on how much elastic and kinetic energy are needed for bump to occur.

4. Explore three hypotheses proposed for mitigating the violent failure of coal specimens. The sudden loss of strength and the degree of ejection and noise were used to judge whether the failure is violent or not. The first hypothesis is that the violent failure occurs because of the sudden failure of the rib zone; the second hypothesis is that the core zone is the main cause for bump; and the third hypothesis is that both the rib and the core zones play a role in the violent failure. The core zone of coal specimens was softened either partially by drilling holes or completely by immersing the specimens in water for 24 hours. It was found that: 
a. Softening the ribs of the coal specimen does not mitigate the violent failure of the coal specimen. While softening the core of the coal specimen eliminates the violent failure, because most of the elastic energy is stored in the core zone, the drilled holes at the core zone allow the core zone to expand laterally and hence store less energy. Once the specimen fails, the released energy from the core zone is not high enough to cause violent failure. Hence, the energy stored in the core zone is the main contribution for the violent failure.

b. Softening specimens with water is not enough to mitigate the violent failures.

5. Since bump prone coal seams are often overlain by a strong and stiff stratum or strata such as sandstone or massive shale. It is necessary to answer the following question: Under static loading conditions, does sandstone, or strong rock, is enough for burst to occur per se? Furthermore, does the mechanics of failure change with changing the strength of the roof and floor? To answer these questions, man-made concrete specimens are assembled in three layered form to investigate the bump mechanics for eight different geological conditions. The reason behind using man-made concrete specimens in this study was that, the ability to examine and determine a specific geological condition contributes to bump using actual coal and rock specimens is not possible, because there is uncertainty about their strength. It was found that:

a. To say a strong roof and floor alone is enough for a burst to occur would be incorrect under static loading conditions. Other factors 
should be taken into consideration as well, such as the width-to-height ratio and the interface friction between the coal specimen and the roof and the floor.

\subsection{General Conclusions:}

1. The potential for violent failure increases with increasing the strength of coal specimen.

2. The coal itself plays an important role in coal mine bumps. However, that role "alone" is not sufficient for the occurrence of bumps.

3. The interface friction and the width-to-height ratio control the mode of failure for coal specimens, and play a substantial role in coal bumps.

4. Under static loading conditions, every interface friction is associated with a specific threshold $\mathrm{W} / \mathrm{H}$ ratio above which the mode of failure is stable and smooth, and below which the failure would be either sudden violent or sudden non-violent.

5. The core zone, the central portion, for the failed coal specimen is not intact as many researchers propose. However, it is damaged to some extent. Most likely the failure of core zone is the main cause for violent failure.

6. Sudden impact load and sudden loss of constraint are associated with instantaneous change in the vertical stress, and sudden release of elastic and kinetic energies. The elastic energy is required for crack initiation and propagation, while the kinetic energy is required for ejecting the debris away from the failed coal specimen. 
7. Softening the rib zone is not effective in mitigating the violent failure of coal specimen. However, softening the core zone help mitigate the violent failure. This gives an indication that the energy stored in the core zone of a coal specimen is the main cause for violent failure, and when this energy released sudden, coal bump happens. Sudden de-confinement is the most likely cause for why it released.

8. Under static or quasi-static loading conditions, to say a strong roof and floor alone is enough for a burst to occur per se would be incorrect, because other factors should also be considered such as, the interface friction between coal and roof and floor and the width-to-height ratio of coal specimen. 


\section{FUTURE WORK IN BUMP AREA}

These points need further investigations:

1. Field investigation and determination for the possible ranges of coal-rock interface. This would require obtaining undisturbed samples including both pillar \& roof and pillar \&floor

2. More work needs to be done to investigate changing the mode of failures at higher interface friction, more than 0.25 .

3. Since elastic strain energy and kinetic energy release when bump occur, future research should focus on determining how much elastic and kinetic energies released when a bump occurs.

4. According to the test results in Chapter seven, "strong roof and floor alone is not enough for bumps to occur under static loading conditions". Therefore future research should investigate dynamic rather than the quasi-static loading condition adopted in Chapter seven. 


\section{REFERENCES}

ABAQUS software (2010). Dassault Systems, providence, RI, U.S.A: http://abaqusdoc.ucalgary.ca/.

Abrams, D. A. (1981). “Design of concrete mixtures”. Lewis Institute, Structural Materials Research Laboratory, Bulletin No. 1, PCA LS001, Chicagohttp://www.portcement.org/pdf_files/LS001.pdf.

Agaito, J.F.T., Goodrich, R. R., and Moon, M. (1997). "Dealing with coal bursts at Deer Creek”. Mining Engineering a Publication of SME, pp. 31-37.

Babcock, C. O. (1985). "Constraint is the prime variable in pillar strength". In: S. S. Peng (Ed.), Proceedings of the $4^{\text {th }}$ International Conference on Ground Control in Mining, Morgantown, WV, pp. 105-116,

Babcock, C. O., and Bickel, D. L. (1984). "Constraint: the missing variable in the coal burst problem". In: S. S. Peng (Ed.), Proceedings of the $3^{\text {rd }}$ International Conference on Ground Control in Mining, held in conjunction with the $25^{\text {th }}$ U.S. Rock Mechanics Symposium. C. H. Dowding and M. Singh (Eds.), Evanston, IL. Northwestern University, pp. 639-647,

Balz, R. and Hucke, A. (2008). "Rock burst prevention in the German coal industry". In: Proceedings of the $27^{\text {th }}$ International Conference on Ground Control in Mining. Morgantown, WV, pp. 46-50

Barron, L. R. (1990). "Longwall stability analysis of a deep, bump-prone western coal mines - case study". In: Proceedings of the $9^{\text {th }}$ International Conference on Ground Control in Mining, Morgantown, WV, pp. 142-149. 
BASF Chemical (2007). Website http://www.anchsand.com/Portals/3/rheomac_product.pdf

BASF Chemical (2007). Website \#2: http://www.bostonsand.com/wpcontent/uploads/2013/11/BASF-Delvo1.pdf.

Brady, G. H. B. and Brown, T. W. (1999). “Rock Mechanics for Underground Mining”. $2^{\text {nd }}$ edition. Norwell, MA, U.S.A. Kluwer Academic. pp. 569.

Brauner, G. (1994). "Rockburst in coal mines and their prevention". Rotterdam, Netherlands. Balkema, A. pp. 144.

Caldarone, M. A. (2009). "High-strength concrete a practical guide" $1^{\text {st }}$ edition. New York, U.S.A. Taylor \& Francis.

Campoli, A. A., Kertis, C. A., and Goode, C. A. (1987). "Coal mine bumps: five case studies in the eastern United States”. U.S. Bureau of Mines, IC 9149, pp. 34.

Chase, F., Zipf, Jr., K., and Mark, C. (1994). "The massive collapse of coal pillars- case histories from the United States". In: Proceedings of the $13^{\text {th }}$ International Conference on Ground Control in Mining. Morgantown, WV, pp. 69-80.

Chen, J. S., Mishra, M., and DeMichiei, J. (1999). "Design considerations for bump-prone longwall mines". In: Proceedings of the $18^{\text {th }}$ International Conference on Ground Control in Mining. Morgantown, WV, pp. 124-135.

Cook, N. G. W. (1963). "The basic mechanics of rockbursts”. Journal of the South African Institute of Mining and Metallurgy, pp. 71-81.

Cook, N. G. W. (1965). “The failure of rock”. International Journal of Rock Mechanics and Mining Science, vol. 2, pp. 389-403. 
Crouch, S. L. and Fairhurst, C. (1937). “The mechanics of coal mine bumps”. Final report submitted to U.S. Bureau of Mines. pp. 88.

Daniels, J., and Moore, L. D. (1907). “The Crushing Strength of Coal”. The Engineering and Mining Journal, pp. 263-268.

DeMarco, M. J. (1996). “Critical pillar concept in yield-pillar-based longwall gateroad design”. Mining Engineering, August, pp. 73-78.

DeMarco, M. J., Koehler, J. R., and Maleki, H. M. (1995). “Gateroad design considerations for mitigation of coal bumps in western U.S. longwall operations”. In: Proceedings: Mechanics and mitigation of violent failure in coal and hard rock mines. U.S. Bureau of Mines, SP 01-95, pp. 141-166.

Gadde, M. M. (2003). "Effect of in-situ stresses on the stability of coal mine development workings". Master's Thesis, West Virginia University, pp. 130.

Garvey, R. and Ozbay, U. (2013). “Assessing coal bumps from excess energy in finite difference models". In: Proceedings of the $32^{\text {nd }}$ International Conference on Ground Control in Mining, Morgantown, WV, pp. 164-168.

GCTS Manual- website: http://www.gcts.com/?s=prod_ver\&p=products\&ID=86.

Griffith, A. A. (1921). "The phenomena of rapture and flow in solids". Phil. Trans. Roy. Soc., A 221, pp. 163-192.

Griffith, W., and Conner, E. T. (1912). "Mining conditions under the city of Scranton, PA". Report and Maps, U.S. Bureau of Mines, Bulletin 25.

Gu, R., and Ozbay U. (2014). “Analyses of de-confinement mechanisms of unstable failures in underground mining conditions". In: Proceedings of the $33^{\text {rd }}$ 
International Conference on Ground Control in Mining. Morgantown, WV, p p. 2226.

Haramy, K. Y. and McDonnel, J. P. (1988). "Control of coal mine bursts". Mining Engineering, pp. 263-268.

Heasley, K. A. (2008). "Back analysis of the Crandall Canyon mine using the LaModel Program". A report submitted to MSHA, http://web.cemr.wvu.edu/ kheasley/LaModelDownloads/Documents/ Papers/MSHA08-CrandallCanyonReport.pdf

Holland, C. T. (1955). Rock Bursts or Bumps in Coal Mines. Colliery Engineering, April, pp. $145-153$.

Holland, C. T. (1958b). "Causes and occurrences of coal mine bumps". SME-AIME Transactions, vol. 221, pp. 994-1004.

Holland, C. T. and Thomas, E. (1954). "Coal mine bumps: Some aspects of occurrence, cause, and controls". U.S. Bureau of Mines, Bulletin 535.

Iannacchione, A. T. and Tadolini, S. C. (2008). "Coal mine burst prevention controls". In: Proceedings of the $28^{\text {th }}$ International Conference on Ground Control in Mining. Morgantown, WV, pp. 20-26.

Iannacchione, A. T. and Tadolini, S. C. (2015). "Occurrence, prediction, and control of coal burst events in the U.S" In: Proceedings of the $34^{\text {th }}$ International Conference on Ground Control in Mining. Morgantown, WV, pp. 36-46.

Iannacchione, A. T. and Zelanko, J. C. (1994). "Pillar mechanics of coal mine bursts: A control strategy". In: Proceedings of the $16^{\text {th }}$ World Mining Congress, vol. 5, pp. $15-23$. 
Iannacchione, A. T. and Zelanko, J. C. (1995). "Occurrence and remediation of coal mine bumps: A historical review" In: Proceedings of mechanics and mitigation of violent failure in coal and hard-rock mines. U.S. Bureau of Mines, SP 01-95. pp. 27-67.

JMP Software, version 11 (2007). SAS Institute Inc., Cary, NC.

Khair, A. W. (1968). "Effect of coefficient of friction on the compressive strength of model coal pillars". M.S. Thesis, West Virginia University. pp. 101.

Lawall, C. E. and Holland., C. T. (1937). "Some physical characteristic of West Virgina coals”. West Virginia University Bulletin, series 37, No. 8. IV, February, pp. 41.

Lawson, H. E., Weakley, A., and Miller, A. (2015). "Dynamic failures in coal seams: implication of coal composition for bump susceptibility". In: Proceedings of the $34^{\text {th }}$ International Conference on Ground Control in Mining. Morgantown, WV, pp. $14-20$.

Maleki H. (1995). "An analysis of violent failure in U.S. coal mines-case studies". In: Proceedings of mechanics and mitigation of violent failure in coal and hard-rock mines. U.S. Bureau of Mines, SP 01-95. pp. 5-26.

Mark, C. (1999). "Empirical methods for coal pillar design". In: Proceedings of the Second International Workshop on Coal Pillar Mechanics and Design. NIOSH IC 9448, pp. $145-154$.

Mehta P. K. and Monteiro P. J. M. (2006). "Concrete microstructure, properties and material". $3^{\text {rd }}$ edition. New York, U.S.A. McGraw-Hill.

Meikle, P. G. (1965). "Effect of friction on strength of model coal pillars". West Virginia University. M.S. Thesis, pp. 61. 
Moore, D. S., Mccabe, G. P., and Craig, B. A. (2009). "Introduction to the Practice of Statistics". $6^{\text {th }}$ edition. New York, NY. U.S.A. W.H. Freeman and Company.

Morsy, K. (2003). "Design consideration for longwall yield pillar stability". Ph.D. dissertation, West Virginia University. pp. 187.

MSHA report (2014). "Coal mine safety and health report of investigation". Fatal rib burst accident. May, 2014. Brody Mine No. 1. I.D. No. 46-09086. pp. 31.

Newman, D. (2002). "A Case history investigation of two coal bumps in the southern Appalachian coalfield". In: Proceedings of the $21^{\text {st }}$ International Conference on Ground Control in Mining .Morgantown, WV, pp. 90-97.

Newman, D. (2008). "Coal mine bumps: case histories of analysis and avoidance". In: Proceedings of the $27^{\text {th }}$ International Conference on Ground Control in Mining .Morgantown, WV, pp. 1-6.

Obert, L. and Duvall, W. (1967). "Rock mechanics and the design of structure in rock". New York, U.S.A. John Wiley \& Sons, Inc. pp. 650.

Osterwald, F. W. (1962). "USGS relates geologic structures to bumps and deformation in coal mine workings". Mining Engineering, vol. 14, April, pp. 63-68.

Ozbay, M. U. (1989). "The stability and design of yield pillars located at shallow and moderate depths". Journal of South African Institute of Mining and Metallurgy, March, pp. 73-79.

Pariseau, W. G. (2008). "Finite element analysis of barrier pillar mining at Crandall Canyon". Report prepared for the Mine Safety and Health Administration, $\begin{array}{llll}\text { Arlington, } & \text { Virginia, } & \text { pp. }\end{array}$ http://www.msha.gov/Fatals/2007/CrandallCanyon/AppendixQ.pdf. 
Pariseau, W. G., Hustrulid, W. A., Swanson, S. R., and Van Sambeek, L. L. (1977). “Coal pillar strength study (the design of production pillars in coal mines)". A final report, U.S. Bureau of Mines, H0242059.

Pen, Y. and Barron, K. (1994). "The role of local mine stiffness in pillar bump prediction”. Rock Mechanics, Nelson \& Laubach (eds). Balkema, Rotterdam, ISBN 905410380 8. pp. 1017-1024.

Peng, S. S. (2008). “Coal mine ground control”. $3^{\text {rd }}$ edition. Morgantown, WV. S. S. Peng. pp. 750.

Peperakis, J. (1958). "Mountain bumps at the Sunnyside mines. Transactions AIME, vol. 211, pp. 982-986.

Prassetyo, S. H. (2010). "The influence of interface friction and W/H ratio on the violence of coal specimen failure”. MS Thesis, West Virginia University. pp. 103

Prassetyo, S. H., Rashed, G., Li, Y., and Peng, S. (2011). "The influence of interface friction and $\mathrm{W} / \mathrm{H}$ ratio on the violence of coal Specimen Failure - A comparison between a bump and non-bump prone mines". In: Proceedings of the $30^{\text {th }}$ International Conference on Ground Control in Mining Morgantown, WV, pp. 92104.

Rashed G. and Peng, S. (2013). "How to mitigate coal mine bumps through understanding of the violent failure of coal specimens". In: Proceedings of the $32^{\text {nd }}$ International Conference on Ground Control in Mining. Morgantown, WV, pp. 1-9.

Rashed, G. and Peng, S. (2015). "How interface friction and width-to-height ratio affect the mode of failure of coal specimens". In: Proceedings of the $34^{\text {th }}$ International Conference on Ground Control in Mining. Morgantown, WV, pp. 21-29. 
Rice, G. S. (1935). "Bumps in coal mines of the Cumberland field, Kentucky and Virginia cause and remedy”. U.S. Bureau of Mine, RI 3267.

Rice, G. S. (1936). "Bumps in coal mines- theories of causes and suggested means of prevention or of minimizing effects”. AIME Transaction (Coal Division), pp. 1139.

Salamon, M.D.G. (1969). "Stability, instability and design of pillar workings". International Journal of Rock Mechanics and Mining Science, vol.7, pp. 613-631.

Salamon, M.D.G. (1984). "Energy considerations in rock mechanics: fundamental results". Journal of South Afr. Inst. Min. Metall., vol. 84, pp. 233-246.

Serata, S. and Nakamura, T. (1985). "Finite element analysis of stress control method". In: Proceedings of the $26^{\text {th }}$ U.S. Symposium on Rock Mech., Rapid City, SD, vol. 26, pp. 659-666.

Singh, S., Sharma, A., and Wei, L. (2008). "Alleviation of rock bursts by identifying burst prone mine workings". In: Proceedings of the $27^{\text {th }}$ International Conference on Ground Control in Mining. Morgantown, WV, pp. 72-78.

SoundPro SE/DL user manual 3M:

http://multimedia.3m.com/mws/media/7755670/soundpro-se-dl-series-sound-level$\underline{\text { meter-user-manual.pdf }}$

Wang, C. K. and Salmon, C. G. (2007). "Reinforced concrete design”. John Wiley \& Sons, Inc. U.S.A.

Wawersik, W. R. and Fairhurst, C. (1970). "A study of brittle rock fracture in laboratory compression experiments.” Int. J. Rock Mech. Min. Sci \& Geomech. Abstr., 7, 561-75. 
Zelanko, J. C. and Heasley, K. A. (1995). "Bump control design protocol for room and pillar coal mining”. In: Proceedings of Mechanics and mitigation of violent failure in coal and hard-rock mines U.S. Bureau of Mines, SP 01-95. pp. 167-180.

Zingano, A., Koppe, J., and Costa, J. (2004). "Violent coal pillar collapse- A case study". In: Proceedings of $23^{\text {rd }}$ International Conference on Ground Control in Mining .Morgantown, WV, pp. 60-67. 


\section{CURRICULUM VITAE}

My name is Gamal Rashed. I received my BS. and my MS. from Geological and Geophysical Engineering Department at Suez Canal University, Egypt. I joined the Mining Engineering Department at WVU in 2010 and worked under Dr. Syd Peng supervision. My area of interest is rock mechanics and ground control. 